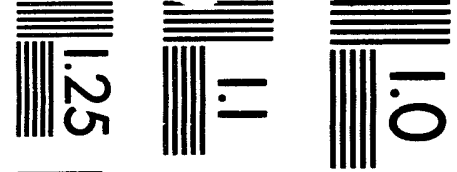

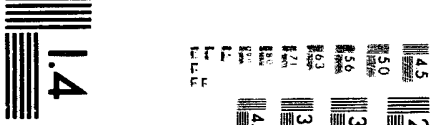

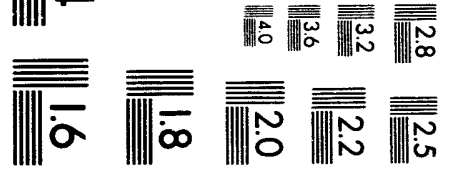



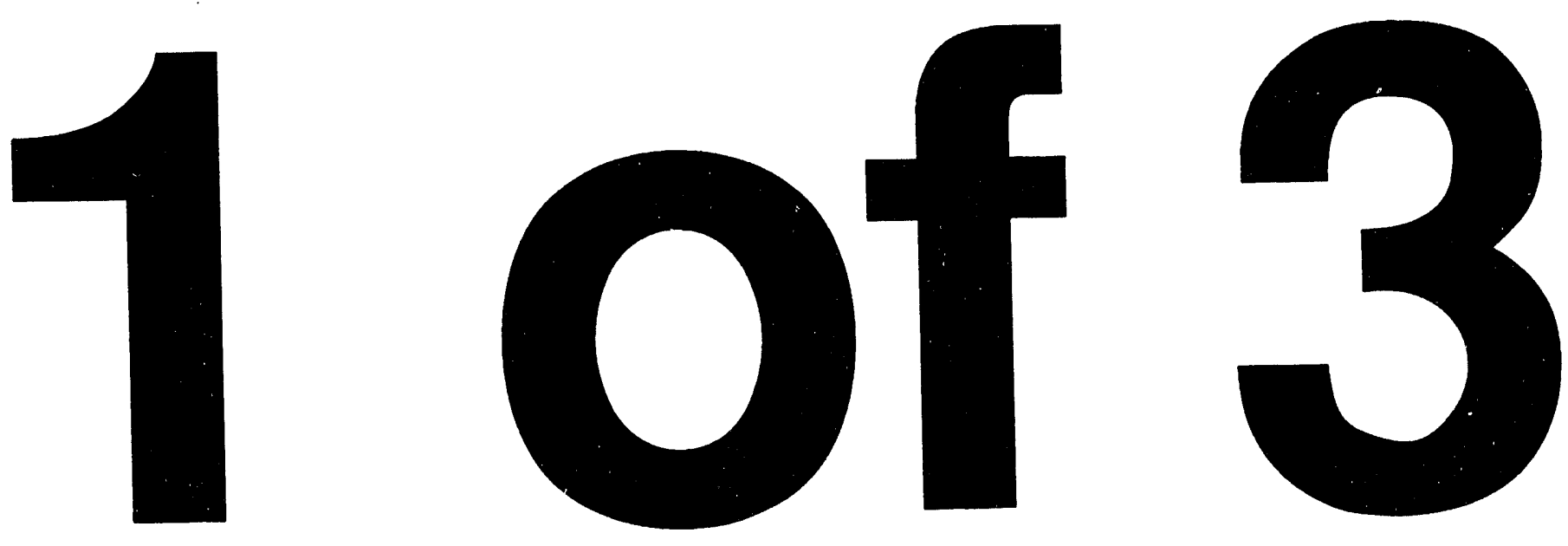


\title{
Directional Solidification studies in Ni-Al Alloys
}

\author{
by Je-hyun Lee
}

\section{thesis submitted to Iowa State University}

\author{
Ames Laboratory, U. S. DOE
}

Iowa State University

Ames, Iowa 50011

Date Transmitted: May 1993

\author{
Prepared for the U. S. Department of Energy \\ Under contract no. w-7405-eng-82.
}

\section{DISCLAIMER}

\begin{abstract}
This report was prepared as an account of work sponsored by an agency of the United States Government. Neither the United States Government nor any agency thereof, nor any of their employees, makes any warranty, express or implied, or assumes any legal liability or responsibility for the accuracy, completeness, or usefulness of any information, apparatus, product, or process disclosed, or represents that its use would not infringe privately owned rights. Reference herein to any specific commercial product, process, or service by trade name, trademark, manufacturer, or otherwise does not necessarily constitute or imply its endorsement, recommendation, or favoring by the United States Government or any agency thereof. The views and opinions of authors expressed herein do not necessarily state or reflect those of the United States Government or any agency thereof.
\end{abstract}


TABLE OF CONTENTS

Page

GENERAL INTRODUCTION

PAPER I. THE PHASE EQUILIBRIA OF $\mathrm{Ni}_{3} A$ Al EVALUATED BY DIRECTIONAL SOLIDIFICATION AND DIFFUSION COUPLE EXPERIMENTS

INTRODUCTION

EXPERIMENTS

8

RESULTS

DISCUSSION

CONCLUSIONS

34

REFERENCES

35

PAPER II. ETCHING CHARACTERISTICS OF Ni33 Al 36

INTRODUCTION

$\begin{array}{ll}\text { EXPERIMENTS } & 39\end{array}$

RESULTS AND DISCUSSION $\quad 42$

$\begin{array}{ll}\text { CONCLUSIONS } & 53\end{array}$

REFERENCES $\quad 55$

PAPER III. THE $\gamma^{\prime} / \gamma^{\prime}$ INTERFACES INDUCED BY FORMATION OF THE $\gamma^{\prime}$ BAND AT THE SOLID-LIQUID INTERFACE DURING QUENCH PROCEDURE

$\begin{array}{ll}\text { INTRODUCTION } & 57\end{array}$

EXPERIMENTS $\quad 59$

$\begin{array}{ll}\text { RESULTS } & 63\end{array}$

$\begin{array}{ll}\text { DISCUSSION } & 119\end{array}$

$\begin{array}{ll}\text { REFERENCES } & 121\end{array}$ 
PAPER IV. CHARACTERISTICS OF EUTECTIC MICROSTRUCTURES NEAR Ni 3 Al COMPOSITIONS

INTRODUCTION

EXPERIMENTS AND RESULTS 128

DISCUSSION 181

CONCLUSIONS 200

REFERENCES 202

GENERAL SUMMARY 204

REFERENCES 207

ACKNOWLEDGEMENTS

208

APPENDIX

209 


\begin{abstract}
Three solid phases are involved in the phase equilibria of the important intermetallic compound $\mathrm{Ni}_{3} \mathrm{Al}$ near its melting point, $\beta, \gamma^{\prime}\left(\mathrm{Ni}_{3} \mathrm{Al}\right)$, and $\gamma$. The generally-accepted phase diagram involves a eutectic reaction between $\gamma^{\prime}$ and $\gamma$, but some recent studies agree with an older diagram due to Schramm, which has a eutectic reaction between the $\beta$ and $\gamma$ phases. The phase equilibria near $\mathrm{Ni}_{3} \mathrm{Al}$ compositions was evaluated using quenched directional solidification experiments, that preserve the microstructures formed at the solidification front, and using diffusion couple experiments. These experiments show that the eutectic forms between the $\beta$ and $\gamma^{\prime}$ phases, as in the Schramm diagram. The growth and phase transformations of these three phases are also studied in the directional solidification experiments. The microstructure analysis shows that the etching characteristics of $\mathrm{Ni}_{3} \mathrm{Al}\left(\gamma^{\prime}\right)$ is very sensitive to small composition variations and crystallographic orientation changes. The eutectic solidification study confirms that the equilibrium eutectic is $\gamma+\beta$, and that the metastable $\gamma+\beta$ eutectic might be also produced in this system according to the impurities, solidification rates, and composition variations.
\end{abstract}




\section{GENERAL INTRODUCTION}

The intermetallic phase of $\mathrm{Ni}_{3} \mathrm{Al}$ has been studied as an important high temperature material. However, the phase diagram near $\mathrm{Ni}_{3} \mathrm{Al}$ compositions was confusing and the growth mechanism of the $\mathrm{Ni}_{3} \mathrm{Al}$ phase was not well known. Three phases are involved in the phase equilibria of the important intennetallic compound $\mathrm{Ni}_{3} \mathrm{Al}$ near its melting point, $\beta(\mathrm{NiAl}), \gamma^{\prime}$ $\left(\mathrm{Ni}_{3} \mathrm{Al}\right)$, and $\gamma(\mathrm{Ni}$ phase). The generally-accepted phase diagram involves a eutectic reaction between $\gamma^{\prime}$ and $\gamma[1,2,3]$, but some recent studies agree with an older diagram due to Schramm, which has a eutectic reaction between the $\beta$ and $\gamma^{\prime}$ phases $[4,5,6]$. A directional solidification study was undertaken to distinguish between the two contradictory phase diagrams near $\mathrm{Ni}_{3} \mathrm{Al}$ compositions.

The reason for the uncertainty might result from the interesting features in this system, a) the composition region of $\gamma^{\prime}$ lies just below the eutectic, b) the temperatures and compositions of the eutectic and peritectic are very close, and c) the solidification temperature is also high enough for solid state diffusion to occur. In this regard, the directional solidification technique is essential to understanding reactions occurring at the solid-liquid interface in this system. This technique preserves the interface structure occurring during the solidification process by quenching of the solidifying interface. The initial study [7] had found strong evidence that the eutectic reaction occurred between the $\beta$ and $\gamma^{\prime}$ phases in contrast to the current diagram accepted by the ASM phase diagram program. In order to further verify that the new phase equilibria was correct additional quenched directional solidification experiments and a series of diffusion couple experiments were done. Also, in order to understand the reactions and phase transformations occurring at the solid-liquid interface near $\mathrm{Ni}_{3} \mathrm{Al}$ compositions the microstructures of three phases were studied at the quenched solid-liquid interface. 
Format Explanation

This thesis consists of four papers (Papers I, II, III, IV) and an appendix. The papers are followed by a General Summary. References cited in the General Introduction follow the General Summary.

In Paper I, the $\mathrm{Ni}_{3} \mathrm{Al}$ phase equilibria in the solid-liquid region has been evaluated using quenched directional solidification technique. The solid phase boundaries just below the eutectic temperature were evaluated using electron probe micro-analysis evaluation of phase boundary compositions in diffusion couple experiments. This paper has been published in Journal of Phase Equilibria Vol.12 No.1 1991. In the directional solidification experiments unusual etching characteristics were found which made interpretation of the microstructures confusing. It was found that the etching characteristics of $\mathrm{Ni}_{3} \mathrm{Al}$ were strongly dependent upon crystallographic orientation by a selected area electron channeling pattern in the SEM in Paper II, which has been published in journal Materials Characterization 29: 285-292(1992). This result was very helpful for interpreting the microstructures of $\mathrm{Ni}_{3} \mathrm{Al}$.

It was interesting to find two $\gamma^{\prime} / \gamma^{\prime}$ interfaces induced by formation of a $\gamma^{\prime}$ band at the solid-liquid interface during the quench procedure. The origin of these interfaces was not well understand and these interfaces have been termed mystery interfaces. Experiments were carried out to understand the origin of the mystery interfaces in Paper III. The crystallographic orientation and structure variations were investigated in the TEM and SACP (Selected Area electron Channeling Pattern) in the SEM. The role of quench rate on the formation of the $\gamma^{\prime}$ band and mystery interfaces was investigated by changing the quench media. A composition change was searched for in the $\gamma^{\prime}$ band region by EDS in both the TEM and SEM.

In Paper IV, the directional solidification experiments of the eutectic structure were continued to confirm the phase diagram of Paper I. The $\gamma^{\prime}+\beta$ eutectic was found, which agrees with the expected phase diagram, but several unusual eutectic structures were observed near the eutectic compositions. These include a three phase eutectic $\left(\gamma^{\prime}+\gamma+\beta\right)$ in a binary alloy, a cellular 
type eutectic where the eutectics formed around the $\gamma^{\prime}$ cells, and large spacings of the eutectics. In order to understand the mechanisms of these unusual eutectic structures formed near $\mathrm{Ni}_{3} \mathrm{Al}$ compositions, directional solidification experiments were done at various velocities with compositions near $\mathrm{Ni}_{3} \mathrm{Al}$. The three phase eutectic might be a real three phase eutectic and a meta-stable eutectic in this system whose solid transformation was very fast. The possibility of the meta-stable eutectic or three phase eutectic was investigated. The effect of impurities on the eutectic was also investigated.

The temperature gradients in the solid and liquid were important parameters in the directional solidification. These gradients were measured at different velocities and at several furnace conditions in Appendix I. The different furnace conditions were obtained by changing the furnace temperatures and the positions of the cold-finger. The gradient data provided important information, not only to study directional solidification, but also to deduce the thermal conductivity of $\mathrm{Ni}_{3} \mathrm{Al}$. 
PAPER I. THE PHASE EQUILIBRIA OF Ni3AI EVALUATED BY DIRECTIONAL SOLIDIFICATION AND DIFFUSION COUPLE EXPERIMENTS 


\section{INTRODUCTION}

There is continued strong interest in the intermetallic compound $\mathrm{Ni}_{3} \mathrm{Al}$ because of its important role in the strengthening of $\mathrm{Ni}$ base superalloys and because single and large grained forms display an increasing strength with temperature while small additions of B have been found to improve room temperature ductility [1]. The accepted high temperature phase equilibria $[2,3,4]$ is shown in Fig. 1, where the phases have been given the conventional names of the superalloy literature, $\gamma, \gamma^{\prime}$, and $\beta$. The liquid-solid equilibria between these three phases involves both a peritectic and a eutectic reaction and the diagram shows the peritectic point occurring at a lower $\mathrm{Ni}$ concentration, $\mathrm{X}_{\mathrm{p}}$, and a higher temperature, $\mathrm{T}_{\mathrm{p}}$, than the eutectic point, $X_{e}$ and $T_{e}$, where the values from Singleton et. al. [4] are given in Table 1. There is older literature data due to Schramm [5] which shows the peritectic point at lower Al concentrations than the eutectic point, and the eutectic forming between the $\beta$ and $\gamma^{\prime}$ phases.

As part of a program to supply single crystal materials, several experiments were carried out using a Bridgeman technique to produce single crystal $\mathrm{Ni}_{3} \mathrm{Al}$. Initial results were confusing and indicated that the Schramm diagram might be correct. In order to more fully understand the reactions occurring at the solid/liquid interface a study was begun to prepare $\mathrm{Ni}_{3} \mathrm{Al}$ by directional solidification techniques that allowed quenching of the solidifying interface. This technique has the advantage that the microstructures formed at the interface are preserved, thereby revealing the nature of the interface structure occurring during the solidification process. In this regard it is interesting to point out that although the phase diagram predicts a eutectic reaction no characteristic eutectic microstructure is observed in conventionally prepared eutectic or near eutectic alloys, presumably due to solid state diffusion on cooling.

Early in this study two papers appeared $[6,7]$ which both supported the Schramm diagram. Hilpert et. al.|6| utilized differential thermal analysis (DTA) to evaluate the liquidus- 


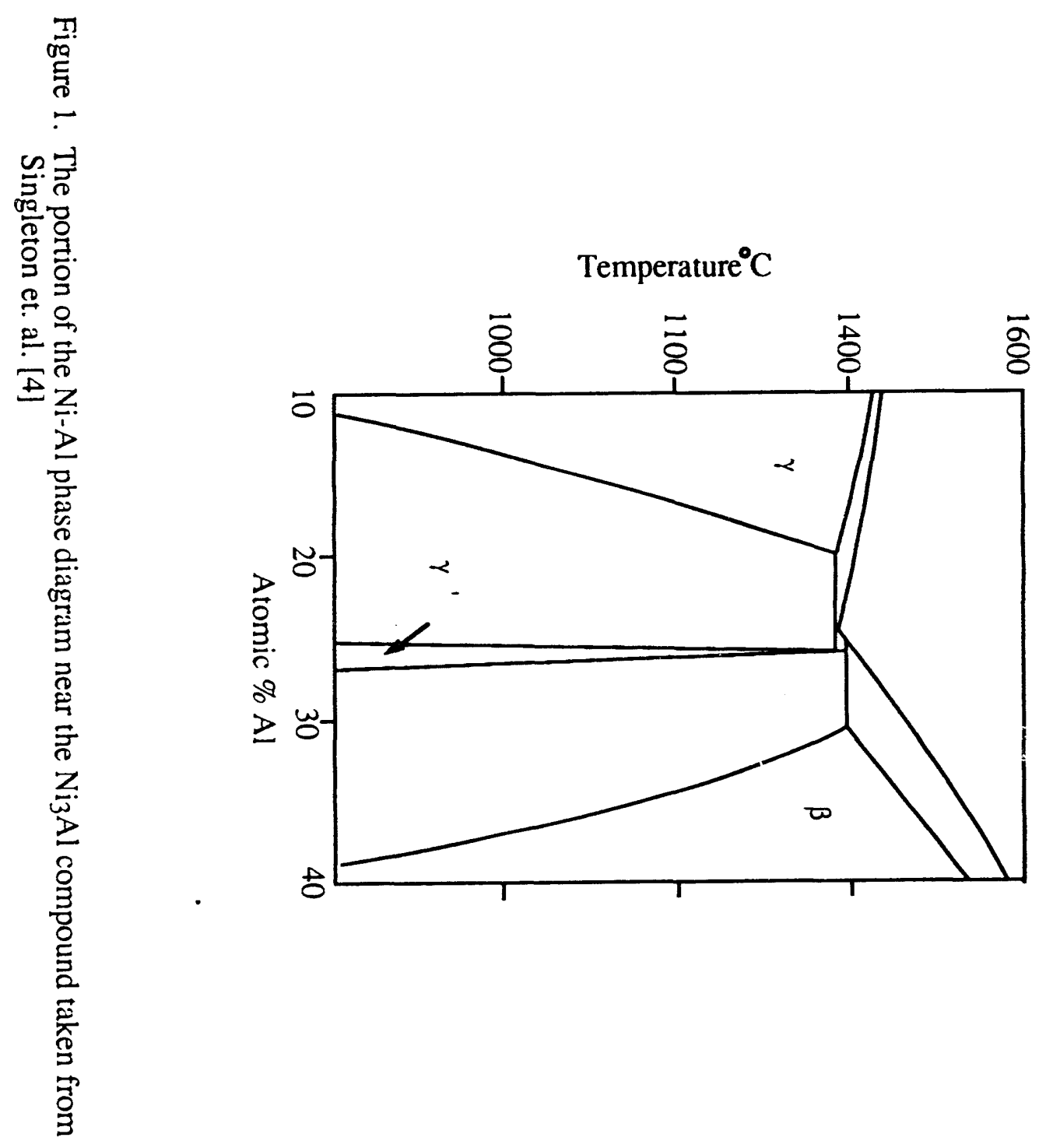

$a$ 
solidus region and obtained 3 points on the phase boundaries below the eutectic temperature using the Knudsen-effusion technique. Bremer et al [7] also used DTA to evaluate the liquidus-solidus region and estimated the lower temperature microstructures from splat cooling and Bridgeman and Czochralski crystal growth experiments. The temperature-composition coordinates of the peritectic and eutectic points from these two studies are included in Table 1 for comparison.

Table 1. Temperature and composition values for the liquidus point of the peritectic and eutectic reactions for $\mathrm{Ni}_{3} \mathrm{Al}$

\begin{tabular}{|c|c|c|c|c|}
\hline Reference & $\mathrm{T}_{\mathrm{p}}\left({ }^{\circ} \mathrm{C}\right)$ & $\mathrm{T}_{\mathrm{c}}\left({ }^{\circ} \mathrm{C}\right)$ & $\mathrm{X}_{\mathrm{p}}($ at.\% Al $)$ & $\mathrm{X}_{\mathrm{e}}($ at. \% Al $)$ \\
\hline \hline Singleton[4] & 1395 & 1385 & 25.5 & 25.0 \\
\hline Hilpert et.al.[6] & 1372 & 1369 & 23.8 & 24.8 \\
\hline Bremer et. al.[7] & 1365 & 1362 & 23.0 & 24.3 \\
\hline This study & $(1383)$ & 1380 & 23.5 & 24.5 \\
\hline
\end{tabular}

In this work the $\mathrm{Ni}_{3} \mathrm{Al}$ phase equilibria in the solid-liquid region has been evaluated using quenched directional solidification techniques, as mentioned above. The phase boundaries just below the eutectic temperature were evaluated using electron probe microanalysis (EPMA) evaluation of phase boundary compositions in diffusion couple experiments. A previous study of $\mathrm{Ni}_{3} \mathrm{Al}$ using inis technique has been carried out by Jannsen 181. The results of this older study were apparently not found in previous evaluations of the phase equilibria $\mid 3,4,6,7)$ and they will be included here. 


\section{EXPERIMENTS}

The alloys were prepared from $\mathrm{Ni}$ and $\mathrm{Al}$ of $99.95 \%$ purity. The temperature measurements were made with Pt-6\%Rh/Pt-36\% Rh (type B) thermocouple wire. Several thermocouples were calibrated by solidifying a 30 gram Au sample within a quartz crucible in the same furnace used for both the directional solidification and the diffusion couple experiments employing the same electrical hook-up and cold junction. The thermocouples consisted of $\mathrm{Al}_{2} \mathrm{O}_{3}$ insulated $0.13 \mathrm{~mm}$ diameter wires inside of $1.5 \mathrm{~mm}$ diameter recrystallized $\mathrm{Al}_{2} \mathrm{O}_{3}$ tubes. The electron probe rnicroanalyses (EPMA) utilized a standard $\mathrm{ZAF}$ software program. To improve accuracy of the analyses single phase $\mathrm{Ni}_{3} \mathrm{Al}$ samples were prepared in the directional solidification apparatus and their composition determined by wet chemical analysis. One of these samples was run with each EPMA examination and used as an internal calibration standard.

\section{Diffusion Couple Experiments}

The apparatus used for the diffusion couple experiments is shown in Fig. 2. The sample was contained inside a quartz tube which was necked down to $8 \mathrm{~mm}$ at the top so that it could be evacuated and back filled with Ar using the same fixture and furnace that was used for the directional solidification experiments. The upper $8 \mathrm{~mm}$ tube was o-ring sealed to the fixture and the experiments were terminated by releasing the seal and quenching the sample in a water container. In the initial diffusion couple experiments pure $\mathrm{Ni}$ was spot welded to the $\mathrm{NiAl}$ (5050 at\%) sample. This technique was only partially successful because the samples usually fractured at the weld interface on quenching. This problem was overcome by using the sample configuration shown in Fig. 2. The NiAl sample was inserted into a Ni can with a piece of pure Ni directly on top of it. The top interface between the pure $\mathrm{Ni}$ and the $\mathrm{Ni}$ can was welded under vacuum in an electron beam welder. The sample was then hot isostatically pressed 


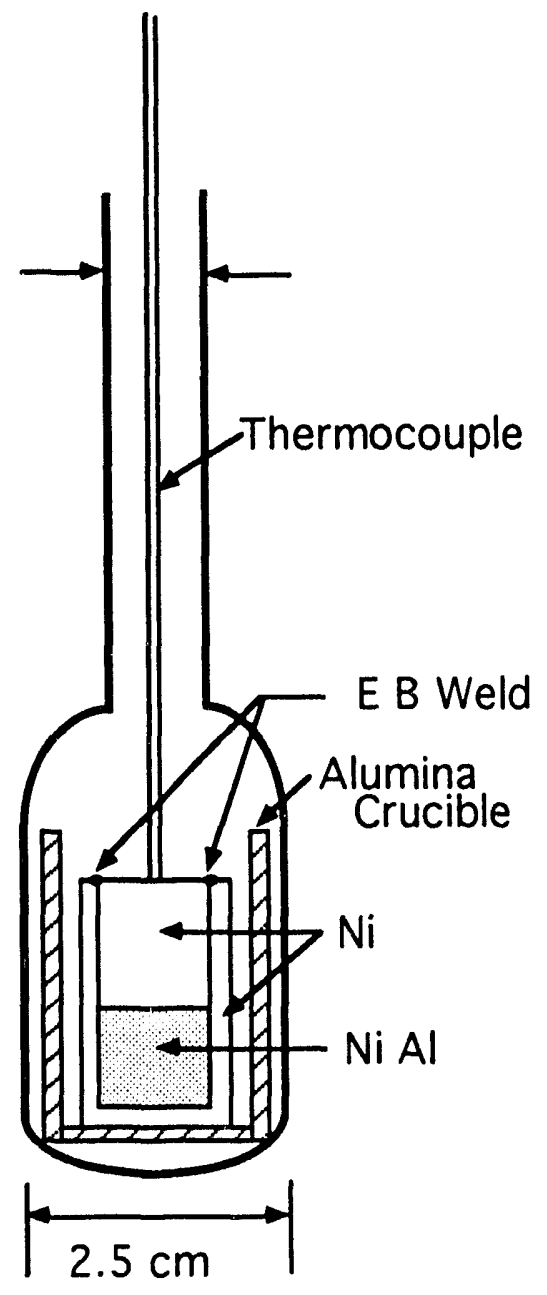

Figure 2. Arrangement used to diffusion anneal the HIP'ed NiAl/Ni couples 
(HIP) at $1000^{\circ} \mathrm{C}$ under $310 \mathrm{MPa}$ pressure for $7 \mathrm{~h}$. This technique proved very successful in producing complete bonding at all $\mathrm{Ni}_{3} \mathrm{Al} / \mathrm{Ni}$ interfaces. The HIP'ed samples were heated at various temperatures in the arrangement shown in Fig. 2 for the diffusion experiments which were terminated by water quenching. The composition profile was evaluated across the sectioned and polished interface regions with EPMA.

\section{Directional Solidification Experiments}

The directional solidification experiments were carried out in $8 \mathrm{~mm}$ OD by $5 \mathrm{~mm} \mathrm{ID}$ recrystalized $\mathrm{Al}_{2} \mathrm{O}_{3}$ tubes. To prepare samples to fit into these tubes pure $\mathrm{Ni}$ and $\mathrm{Al}$ were vacuum/inert gas melted in $\mathrm{Al}_{2} \mathrm{O}_{3}$ crucibles utilizing low frequency $(3 \mathrm{kHz})$ induction heating to produce good mixing. After holding for $10 \mathrm{~min}$ at around $1450^{\circ} \mathrm{C}$ under an $\mathrm{Ar}$ atmosphere of 680 Torr , a vertical array of nine quartz tubes of $4.0 \mathrm{~mm}$ I.D. was lowered so that the open bottom ends of the tubes penetrated below the melt surface. The upper ends of the tubes were sealed into a fixture with a refractory cement and connected to a vacuum pump through a quick release valve. Immediately after lowering into the melt, the quick release valve was opened, causing the melt to be drawn up into the nine quartz tubes to a height of $\approx 40 \mathrm{~cm}$. The rods were then raised above the melt surface and solidified.

The directional solidification apparatus is shown schematically in Fig. 3. The sample was held in the bottom of the $\mathrm{Al}_{2} \mathrm{O}_{3}$ containment tube which was closed at the bottom and open at the top and connected there with an o-ring seal to the vacuum/inert gas system. A thermocouple or a probe rod was inserted through another o-ring seal and could be moved up and down through the column of liquid metal above the solid-liquid interface. The $\mathrm{Pt} / \mathrm{Rh}$ furnace melted the alloy down to a point near the bottom of the furnace. The carriage + cold finger + furnace were raised upward on three ball bushing guide rods at a rate controlled by a computer connected to a stepper motor. To make a run the sample was heated up first under vacuum and when the temperature reached around $1200^{\circ} \mathrm{C}$ an atmosphere of $680 \mathrm{Torr}$ Ar was 


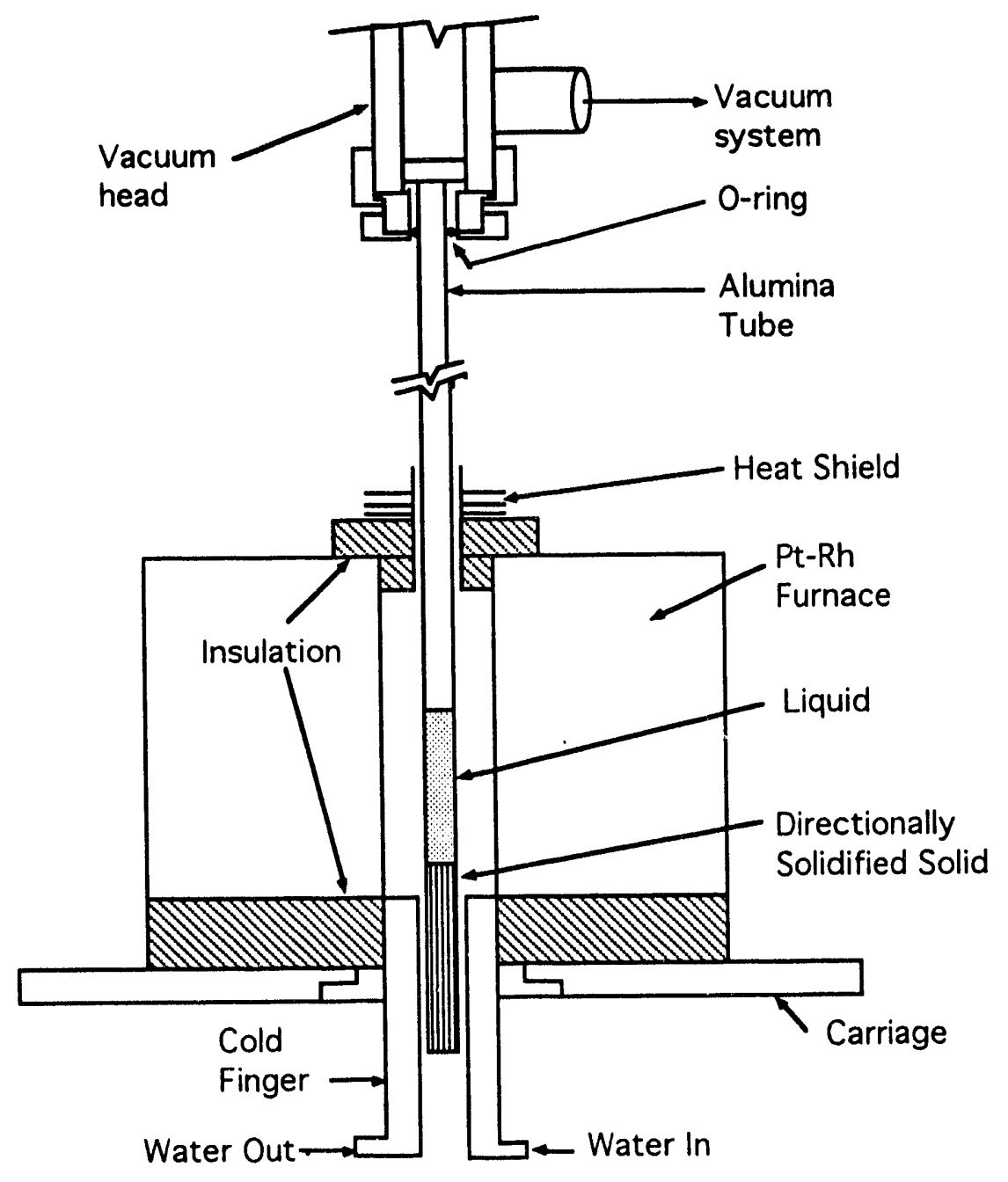

Figure 3. Schematic of the directional solidification apparatus used in this study 
introduced. The furnace temperature was held at $1500^{\circ} \mathrm{C}$ for around 45 minutes during which time it was necessary to run either the $1.5 \mathrm{~mm}$ diam. thermocouple assembly, or an $\mathrm{Al}_{2} \mathrm{O}_{3}$ probe of the same size, down to the interface in order to cause bubbles of trapped gas to rise out of the column of liquid. After the carriage had been raised at the desired rate for the desired distance the upper o-ring seal on the $\mathrm{Al}_{2} \mathrm{O}_{3}$ tube was released and the tube dropped through the cold finger into a water quench bath. The samples were then sectioned longitudinally, polished and examined using optical microscopy and analytical EPMA scanning microscopy.

In order to evaluate the temperature gradient in the system the tip of the thermocouple was lowered to a position $15 \mathrm{~mm}$ above the advancing solid/iquid interface. The output of the thermocouple was measured at short time intervals and stored in a computer while the carriage was raised at a constant rate. Unless otherwise noted all of the experiments reported here were done at a solidification rate of $61 \mu \mathrm{m} / \mathrm{s}$. 


\section{EXPERIMENTAL RESULTS}

\section{Diffusion Couple Experiments}

Figure 4 presents a micrograph of the interface region in the $1249^{\circ} \mathrm{C}$ diffusion couple experiment. The sample has been etched and the regions of the three phases $\gamma, \gamma^{\prime}$, and $\beta$ are all clearly seen. Only the $\gamma^{\prime}$ region is single phase. A very fine precipitate of $\gamma^{\prime}$ (not visible on Fig.4) has formed on quenching in the $\gamma$ region on the left of the photo. The $\beta$ region on the right of the photo is composed of three distinct regions. At the left side it is $\beta+\gamma$ precipitates, at the right it is $\beta$, and in between it is $\beta$ martensite Experiments have shown [9] that the $M_{S}$ temperature of the $\beta$ phase is composition dependent and it lies above room temperature at compositions above 37 at. $\% \mathrm{Al}$. The composition measured at the $\beta$ martensite/ $\beta$ transition on Fig.4, was close to this value, see Fig. 5.

The EPMA work was done on unetched surfaces to insure a constant local angle between the electron beam and sample surface. The interface location was not visible on the polished surfaces and this problem was overcome by making microhardness indentations along the interface which were not completely removed in the subsequent polishing operation. A line scan about $40 \mu \mathrm{m}$ long running parallel to the diffusion interface was used to average over the fine precipitate phases in the $\gamma$ and $\beta$ regions. Figure 5 presents the EPMA analysis of the sample of Fig. 4 as an example of experimental data. The interface compositions in neighboring phases were evaluated from the measured compositions at the phase boundaries and the results are presented in Table 2. Four temperatures were studied with times ranging from $48 \mathrm{~h}$ at $1200^{\circ} \mathrm{C}$ to $5 \mathrm{~h}$ at $1340^{\circ} \mathrm{C}$. Two EPMA analyses were done at different locations along the diffusion couple interface for each sample and the average difference of the measured interface compositions was 0.11 at\% $\mathrm{Al}$. 


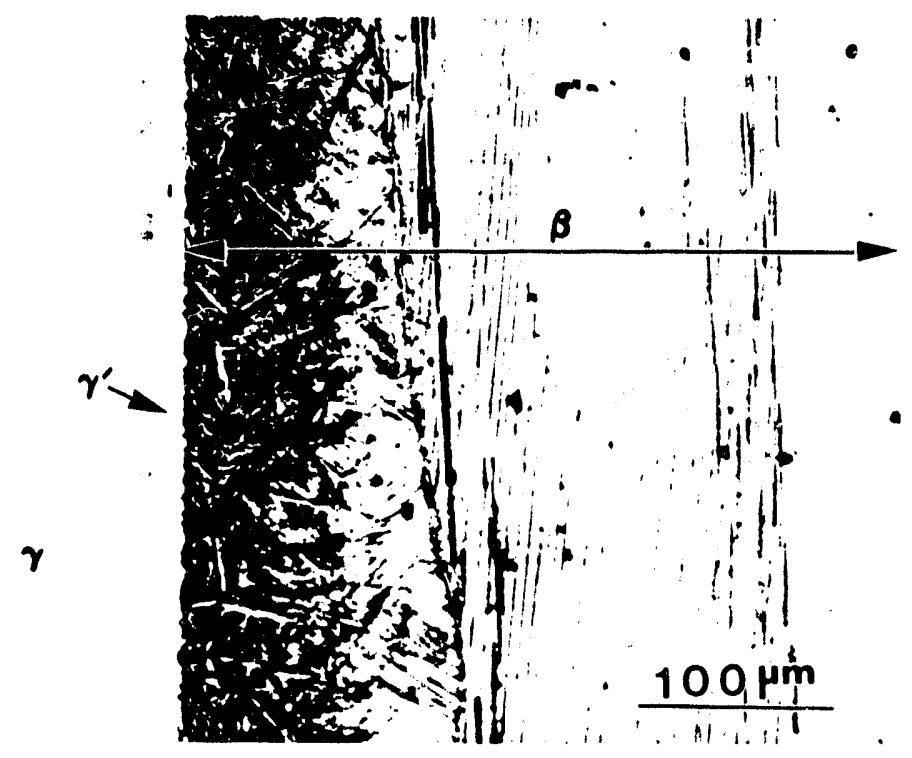

Figure 4. Etched surface (Marbles etch) of the interface region of the diffusion couple experiment run at $1249^{\circ} \mathrm{C}$ for $18 \mathrm{~h}$. Original mag. $=200 \mathrm{x}$ 


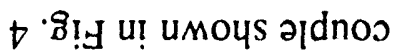

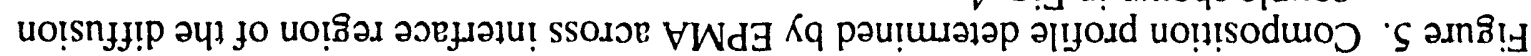

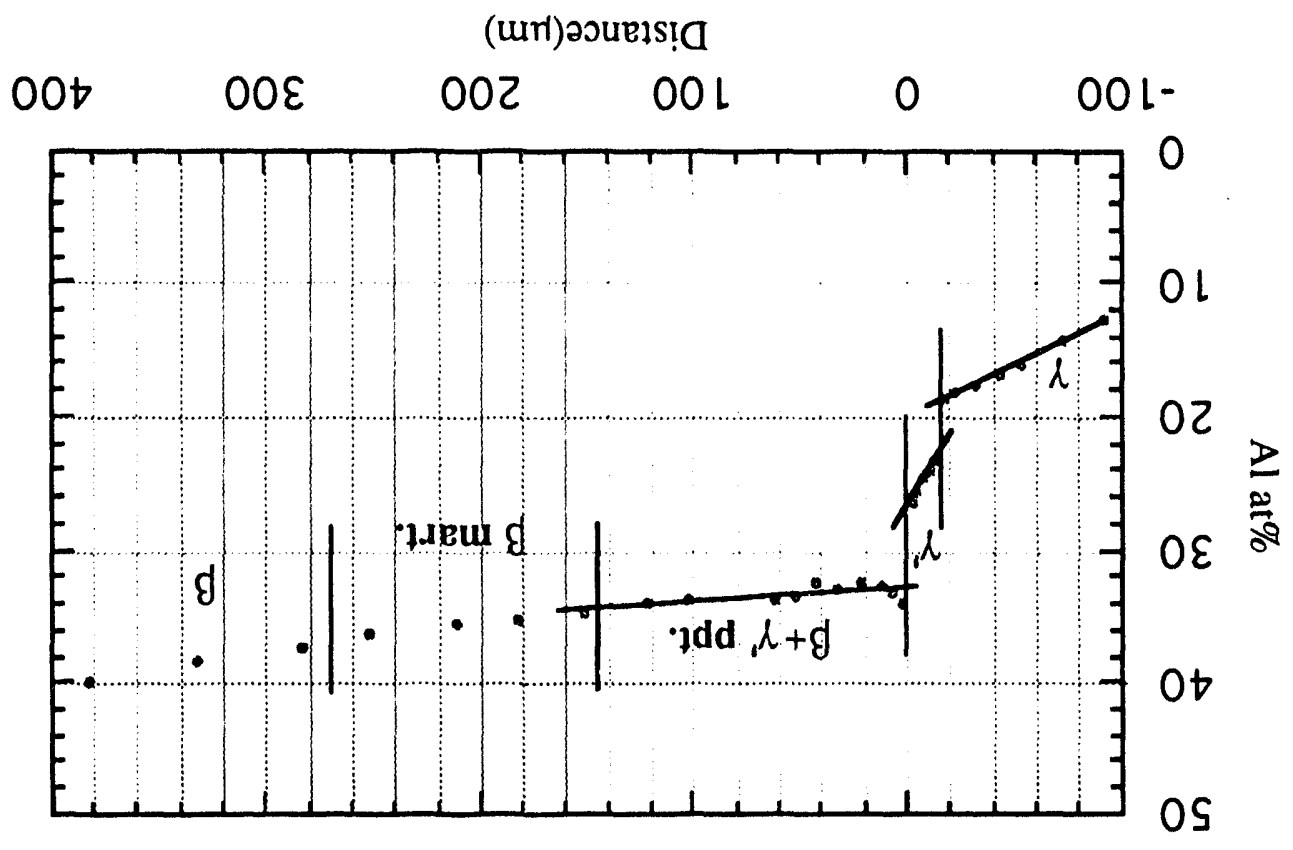


Table 2. The phase boundary compositions of the diffusion couples determined by EPMA

\begin{tabular}{|c|c|c|c|c|}
\hline \multirow{2}{*}{$\begin{array}{c}\text { Temperature } \\
\left({ }^{\circ} \mathrm{C}\right)\end{array}$} & \multicolumn{4}{|c|}{ Boundary composition (at.\%Al) } \\
\cline { 2 - 5 } & $\beta$ and $\beta+\gamma^{\prime}$ & $\beta+\gamma^{\prime}$ and $\gamma^{\prime}$ & $\gamma^{\prime}$ and $\gamma+\gamma^{\prime}$ & $\gamma+\gamma^{\prime}$ and $\gamma$ \\
\hline \hline 1355 & 28.9 & 25.5 & 22.7 & 20.7 \\
\hline 1340 & 29.5 & 25.3 & 23.0 & 20.1 \\
\hline 1249 & 32.2 & 26.2 & 23.4 & 18.5 \\
\hline 1200 & 34.4 & 25.5 & 23.5 & 17.3 \\
\hline
\end{tabular}

\section{Directional Solidification Experiments}

For all five target compositions studied, 23, 24, 25, 26, 27 at\% Al, at the solidification rate of $61 \mu \mathrm{m} / \mathrm{s}$, the quenched interface regions revealed a dendritic microstructure. As shown in Table 3 a eutectic microstructure consisting of $\beta+\gamma^{\prime}$ phases was found at the base of the

Table 3. Characteristics of the dendrite tip region revealed by the quenched microstructures

\begin{tabular}{|c|c|c|c|c|c|}
\hline At $\% \mathrm{Al}$ & 27 & 26 & 25 & 24 & 23 \\
\hline $\begin{array}{l}\text { Phases in Dendrite Tip } \\
\text { Region } \\
\end{array}$ & $\beta+\gamma$ & $\beta+\gamma$ & $\beta+\gamma$ & $y$ & $\underline{\gamma}$ \\
\hline Dendrite Length (mm) & 9.6 & 6.9 & 0.8 & 0.5 & 3 \\
\hline $\begin{array}{c}\text { Interdendritic Structure } \\
\text { at Dendrite Base }\end{array}$ & Eut $\left(\beta+\gamma^{\prime}\right)$ & $\operatorname{Eut}\left(\beta+\gamma^{\prime}\right)$ & $\begin{array}{c}\text { Eut }\left(\beta+\gamma^{\prime}\right) \\
+\gamma^{\prime}\end{array}$ & $\begin{array}{c}\text { Eut }\left(\beta+\gamma^{\prime}\right) \\
+\gamma^{\prime}\end{array}$ & $\operatorname{Eut}\left(\beta+\gamma^{\prime}\right)$ \\
\hline
\end{tabular}

dendrites with all five compositions, and the lengths of the primary dendrites above the eutectic growth front are also listed in the table. There is a switch in the structure of the dendrites from the $\beta$ phase at 25 to 27 at $\%$ Al to the $\gamma$ phase at 23 and 24 at\% Al. The quench was not fast enough to prevent the $\gamma^{\prime}$ phase from forming as a precipitate in the $\beta$ phase dendrites of the 25 to 27 at\% Al alloys. 
Figure 6 shows the eutectic growth front for the alloy of 25 at\% Al. The quenched liquid region is at the top and the side branches of two vertically growing primary dendrites appear at either side of the photo. Higher magnification examination shows the dendrites to be composed of $\beta+\gamma^{\prime}$ phases. Phase identification was done with EPMA. The Marbles etch caused the $\beta$ phase to appear dark and both the $\gamma$ and $\gamma^{\prime}$ phases white. The eutectic growth front appears cusped with an indication that the $\gamma^{\prime}$ phase (the light phase inside the eutectic) is forming at the base of the cusps. Figure 7 is a slightly lower magnification picture located below the position of Fig. 6 such that the top of Fig. 7 is $2.1 \mathrm{~mm}$ below the eutectic growth front and the bottom lies $4.4 \mathrm{~mm}$ below. It is seen that the eutectic microstructure has completely decomposed into $\gamma$ ' phase during the cooling from the eutectic formation temperature to the temperature at the position $4.4 \mathrm{~mm}$ below the interface. The growth front for the 24 at $\% \mathrm{Al}$ alloy is shown in Fig. 8. In this case the primary dendrites are single phase $\gamma$ and the structure at the base of the primaries again consists of both the $\beta+\gamma^{\prime}$ eutectic plus primary $\gamma^{\prime}$, but now the $\gamma^{\prime}$ is a larger volume fraction and at some locations it is cusped upward with the cusp tips growing at a slightly higher temperature than the eutectic front. In the remaining three alloys, 23,26 and 27 at $\% \mathrm{Al}$, the structure at the base of the dendrites was all $\beta+\gamma^{\prime}$ eutectic with a microstructure identical to the eutectic microstructures seen on Figs. 6 and 8.

The structure of both the $\beta$ dendrites and the $\beta+\gamma^{\prime}$ eutectic is shown in Fig. 9, where the picture is located very close to the eutectic growth front. To assist in identifying the phases here the compositions determined at the labelled positions using EPMA are given in Table 4. The $\beta$ dendrites have formed $\gamma$ precipitate throughout even up to the tips of the dendrites. Because of the difficulty in obtaining a true average composition over two phases with EPMA it was not possible to obtain an estimate of the solidus composition at the $\beta$ dendrite tips. The $\beta$ dendrites were examined in all of the alloys and the results were all similar.

In the $\beta+\gamma^{\prime}$ eutectic the $\beta$ phase is the minor phase and it consistently gave 


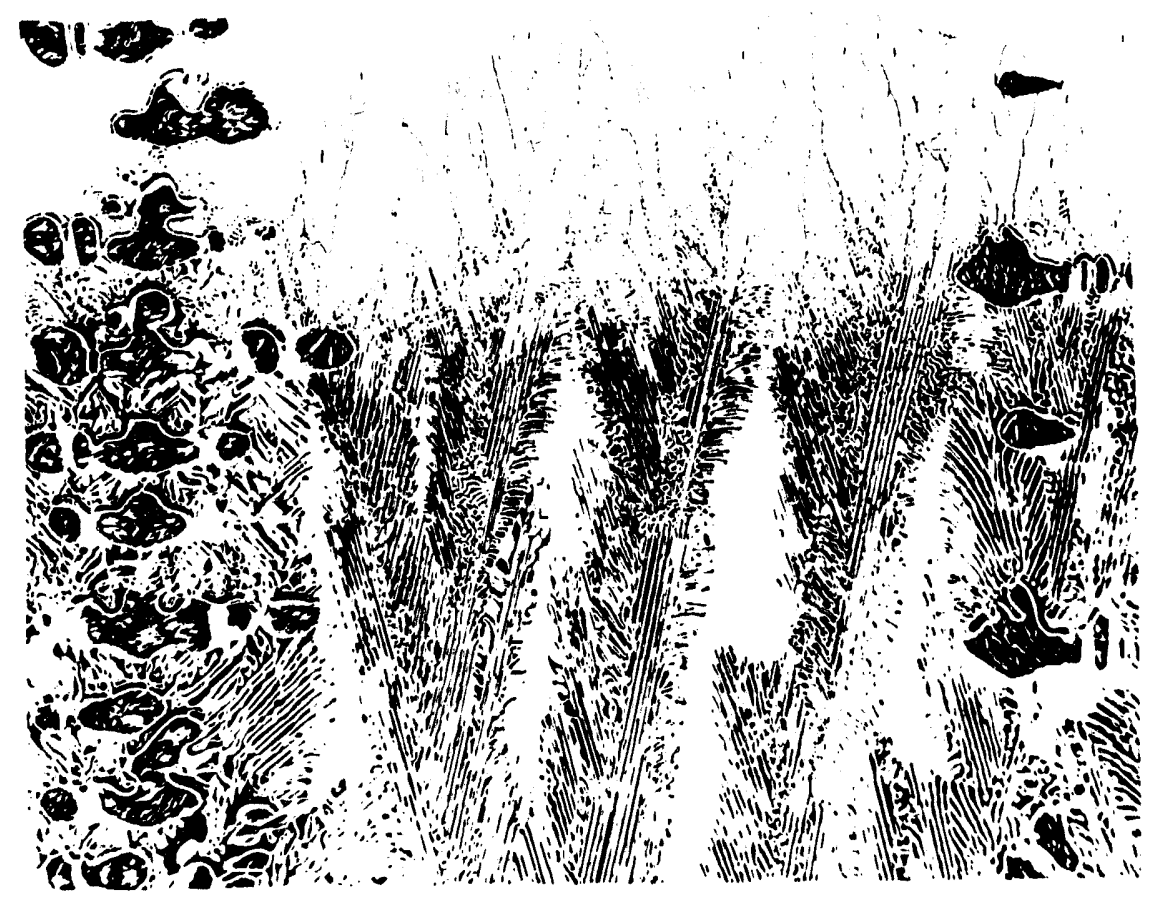

Figure 6. Longitudinal section of eutectic growth front in the 25 at\% Al alloy. Growth direction is vertically upward. Marbles etch. Original Mag. $=100 \mathrm{X}$ 

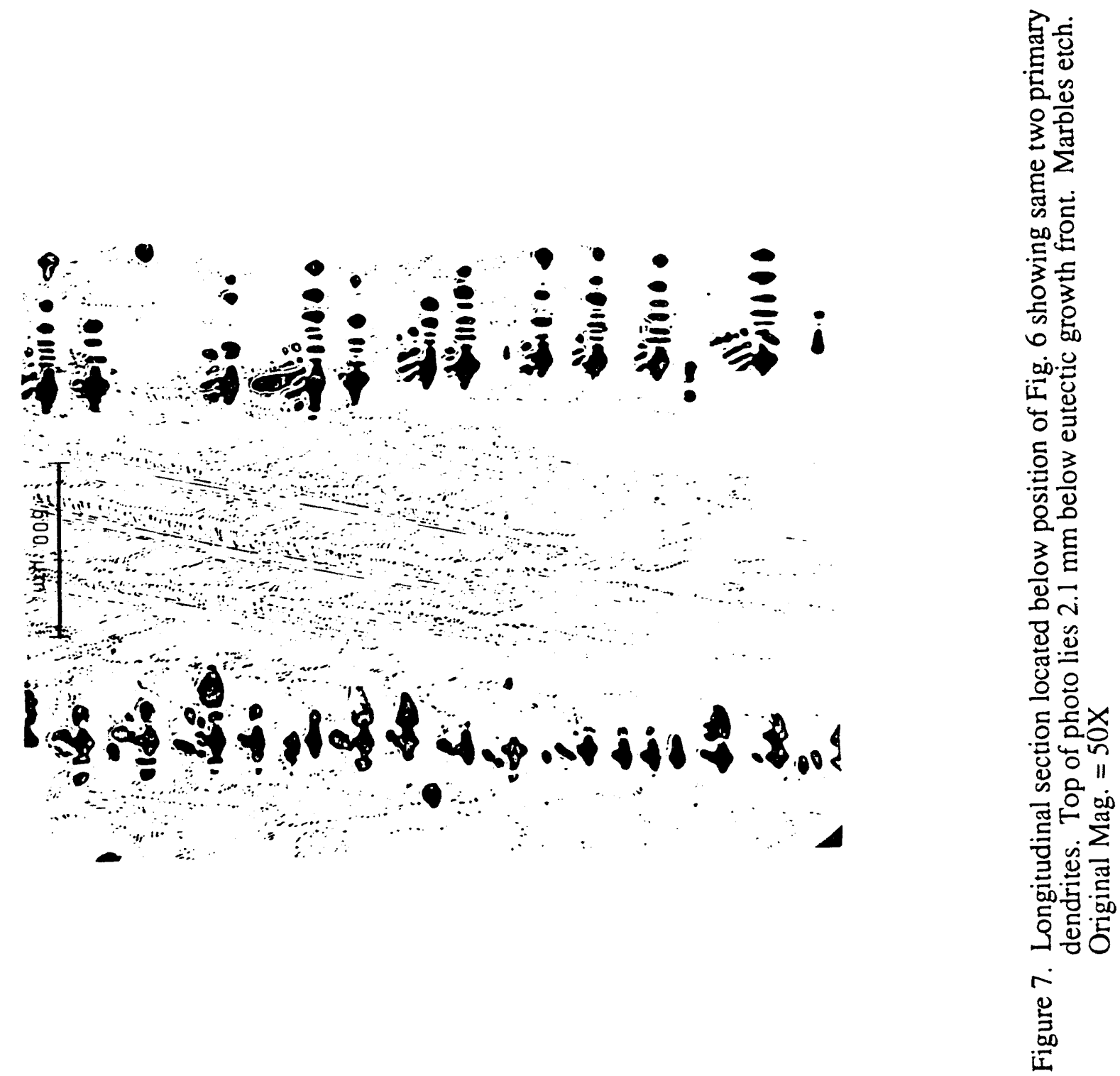


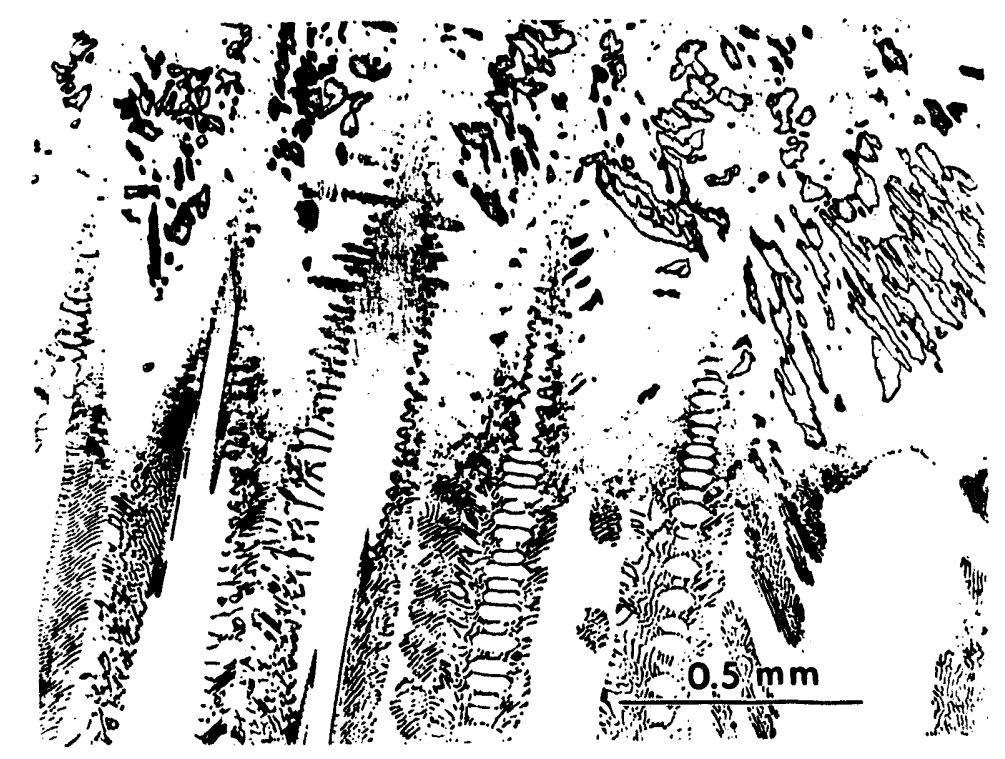

Figure 8. Longitudinal section of eutectic growth front in the 24 at\% Al alloy. Growth direction is vertically upward. Marbles etch. Original Mag. $=50 \mathrm{X}$ 


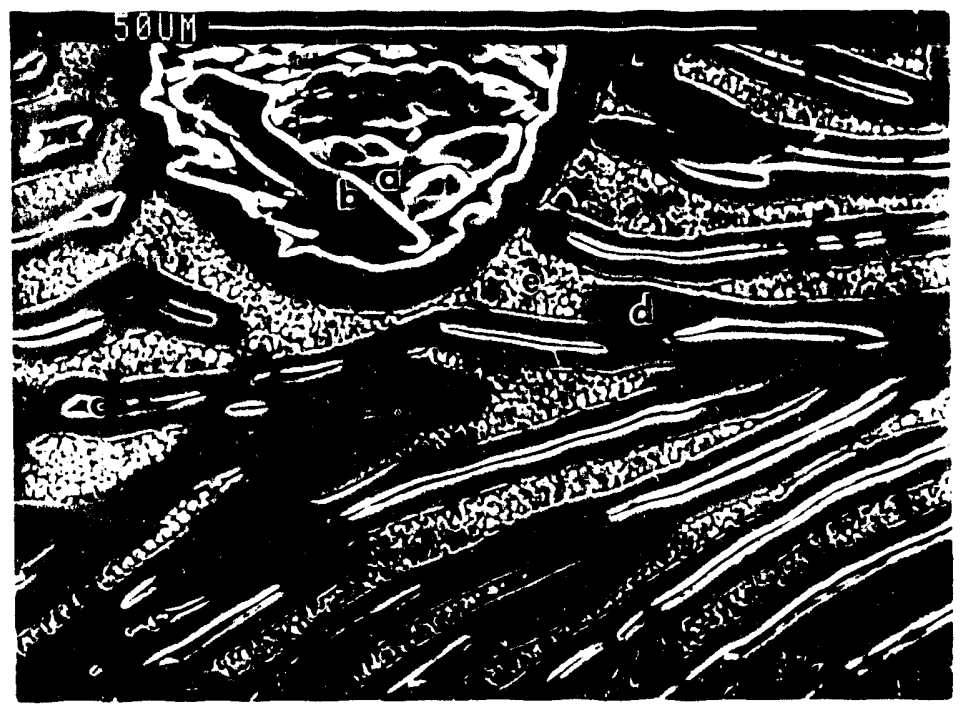

Figure 9. Quenched interface region of 25 at $\% \mathrm{Al}$ alloy locating the five types of positions that were analyzed by EPMA. Marbles etch. Original Mag. $=1020 \mathrm{X}$ 
Table 4. The average compositi- as determined by EPMA on the five types of positions shown by labels a to e on Fig. 9

\begin{tabular}{|c|c|c|c|}
\hline Location & Position & At \% Al & Phase \\
\hline \hline Dendrite - dark & $\mathrm{a}$ & 33.5 & $\beta$ \\
\hline Dendrite - light & $\mathrm{b}$ & 25.5 & $\gamma^{\prime}$ \\
\hline Eutectic - Minor Phase & $\mathrm{c}$ & 33.3 & $\beta$ \\
\hline Eutectic - Edge Major. Ph. & $\mathrm{d}$ & 24.7 & $\gamma^{\prime}$ \\
\hline Eutectic - Center Major Ph. & $\mathrm{e}$ & 21.5 & $\gamma^{\prime}+\gamma$ \\
\hline
\end{tabular}

compositions of around $33.5 \mathrm{at} \% \mathrm{Al}$. The $\gamma^{\prime}$ major phase was consistently found to have formed $y$ precipitates at the center of the lamellae as is seen at point e in Fig 9. This structure was found in all five alloys and Fig. 10 presents the EPMA compositions at the three characteristic regions of the eutectic in the five alloys, measured at positions located very close to the eutectic growth front. The analyses were made on samples etched only very lightly to avoid surface rounding, and regions having a width of $3 \mu \mathrm{m}$ or greater were selected for analysis to avoid problems from beam spreading.

The quench was rapid enough to avoid precipitation of $\gamma^{\prime}$ in the tip regions of the $\gamma$ dendrites and it was therefore possible to evaluate the composition of the solid forming at the dendrite tips in the alloys of 23 and 24 at\% Al, which were found to average, 21.2 at\% $\mathrm{Al}$ and 20.3 at\% $\mathrm{Al}$, respectively.

Important questions for the interpretation of the compositional results are, (a) how close are the actual compositions to the target compositions, and (b) is macrosegregation during solidification adequate to produce significant changes in composition with length along the sample? To answer these questions samples were sectioned above (Sample $\# 2$ ) and below (Sample \#1) the final quench interface as shown in Fig. 11 and analyzed by wet chemical analysis. The precision of this analytical technique was evaluated by dividing sample 2 into 


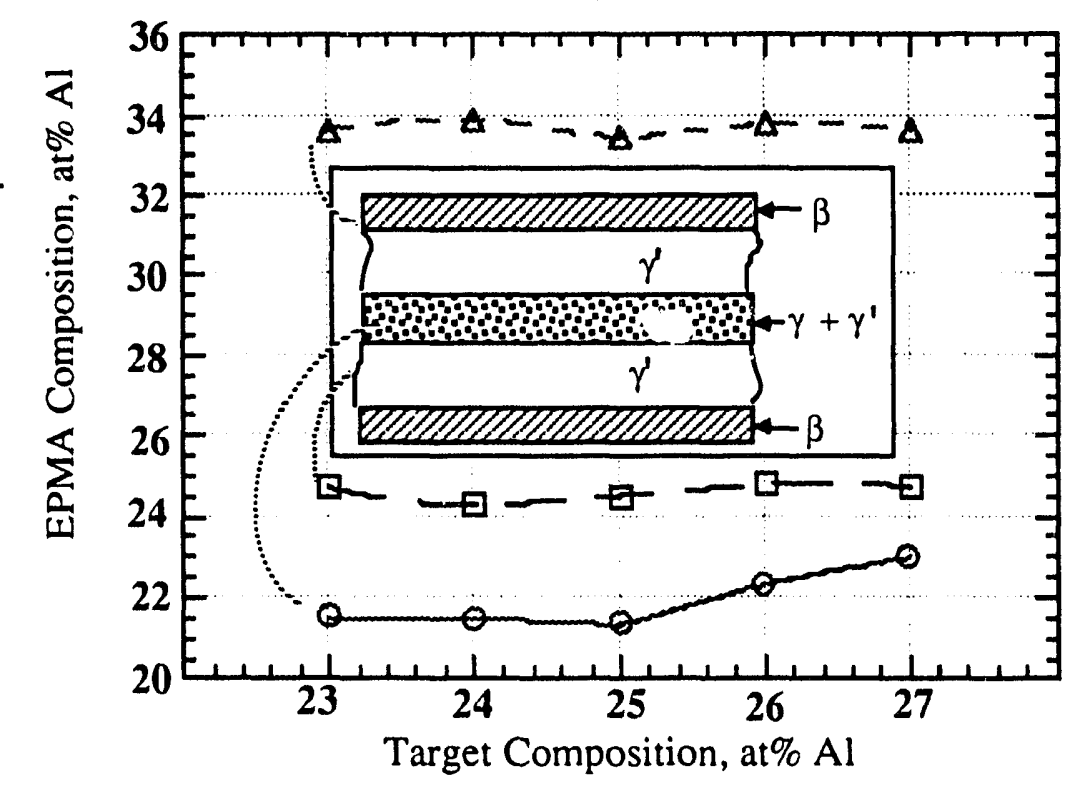

Figure 10: The average compositions of the three regions in the $\beta+\gamma^{\prime}$ eutectic structures of all five alloys 


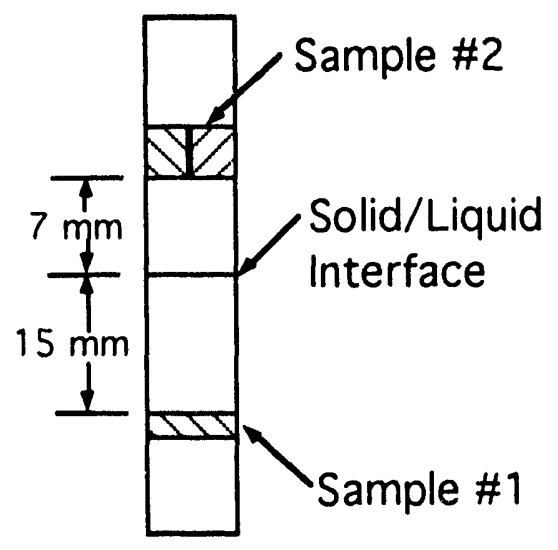

Figure 11 The location of samples removed for wet chemical analyses 
two sections and comparing the analyses. The average analyses over two such sections should be the same as the circumferential symmetry of the system would not be expected to produce circumferential composition differences. The results from two samples (not otherwise presented here) gave 23.74 versus 23.85 and 24.09 versus 24.16 at\% Al, which indicate that the precision is on the order of 0.1 at\% Al. The results of the analyses on the three samples of 23, 25 and 27 at\% $\mathrm{Al}$ are presented in Table 5.

Table 5. Wet chemical analysis at locations \#1 and \#2 shown on Fig. 11 for three samples

\begin{tabular}{|c|c|c|c|}
\hline Target at\% Al & Sample \#1 & Sample \#2 & $\Delta$ \\
\hline \hline 23 & 22.59 & 23.03 & -0.44 \\
\hline 25 & 24.97 & 24.91 & +0.04 \\
\hline 27 & 26.82 & 26.81 & +0.01 \\
\hline
\end{tabular}

The difference in the values of samples 1 and 2 are shown in the column labelled $\Delta$ and it is seen that essentially no macrosegregation has occurred in the 25 and 27 at\% $\mathrm{Al}$ alloys, but that a small amount may have occurred in the 23 at\% Al alloy. Nevertheless, comparing the values of sample \#2 to the target compositions it is seen that the dendrite tips are growing into liquid with a composition within 0.1 at \% Al of the target composition in the 23 and 25 at\% $\mathrm{Al}$ alloys and within $0.2 \mathrm{at} \% \mathrm{Al}$ in the $27 \mathrm{at} \% \mathrm{Al}$ alloy.

The temperature profile in the liquid was evaluated at solidification rates from 1 to $1(0)$ $\mu \mathrm{m} / \mathrm{s}$ and it was found that the latent heat contribution had a significant effect. The profile for the rate used in this study, $61 \mu \mathrm{m} / \mathrm{s}$ is shown in Fig. 12. The break in the curve at the eutectic growth front became less sharp at lower rates and more sharp at higher rates due to the latent heat effect. The eutectic growth front temperature, evaluated by extrapolating the curves to the break in slope, was 1377,1380 and 1380 at the growth rates of 61,80 and $100 \mu \mathrm{m} / \mathrm{s}$. Because 
the breaks were sharper at the higher rates the values of $1380^{\circ} \mathrm{C}$ appears to be the best estimate of the eutectic growth temperature in these experiments. At the $61 \mu \mathrm{m} / \mathrm{s}$ rate the liquid temperature gradient was $4.2^{\circ} \mathrm{C} / \mathrm{mm}$ and the solid gradient was $2.2^{\circ} \mathrm{C} / \mathrm{mm}$. 


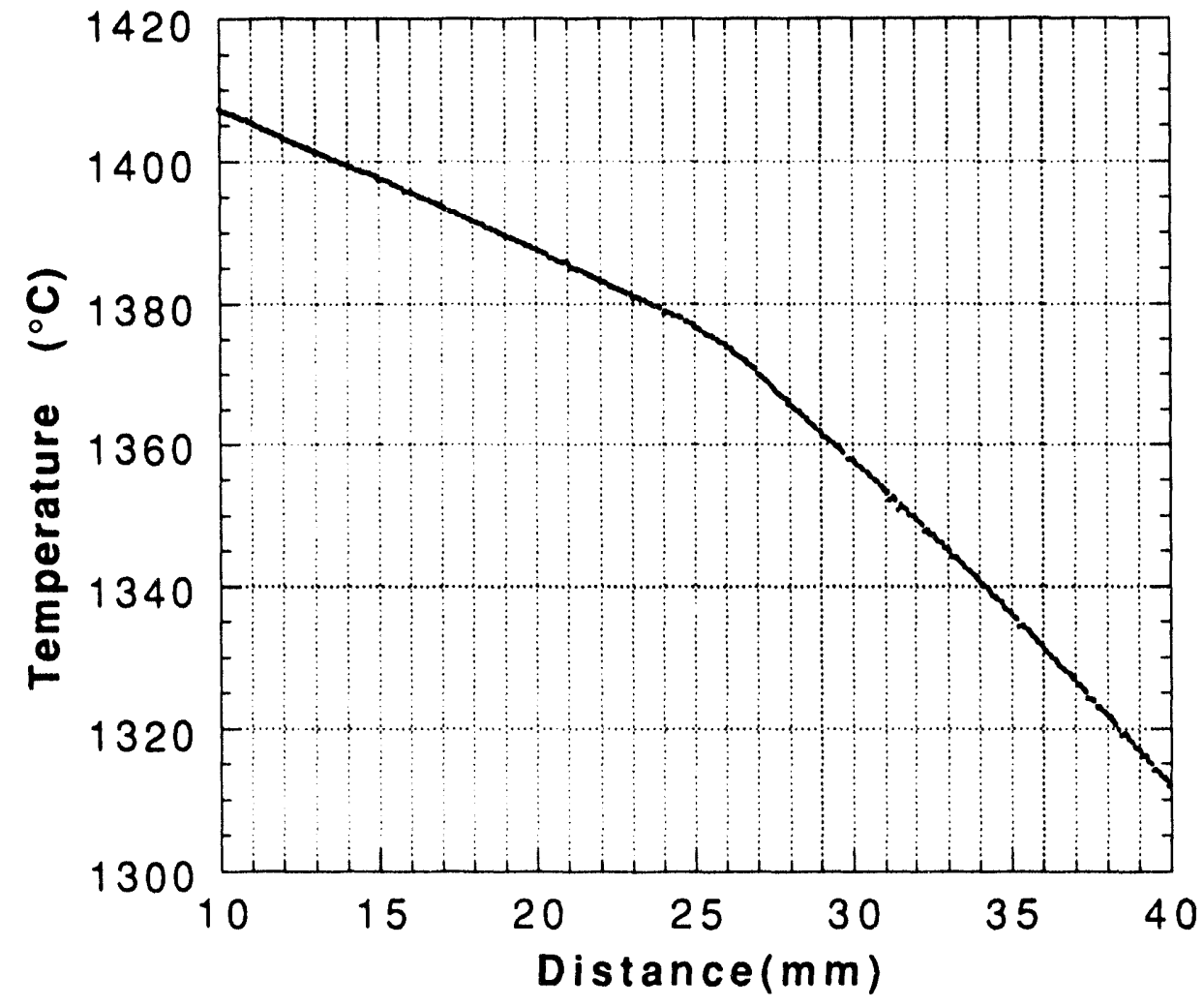

Figure 12. The temperature profile measured on a $24 \mathrm{at} \% \mathrm{Al}$ alloy solidified at $61 \mu \mathrm{m} / \mathrm{s}$ 


\section{DISCUSSION}

Figure 13 presents the phase diagram based on the data of this study along with the solid state data of Hilpert [6] and the diffusion couple data of Jannsen [8]. Agreement of the present data with this previous work $[6,8]$ on the four solid state phase boundaries appears to be reasonably good. The fact that a eutectic microstructure composed of $\beta+\gamma^{\prime}$ occurs at the base of the dendrites at all compositions from 23 to 27 at\% $\mathrm{Al}$ is conclusive evidence that at the solidification rate studied here, $61 \mu \mathrm{m} / \mathrm{s}$, the phase formation follows the diagram of Fig. 13 and not that of Singleton et. al., Fig. 1. If Fig. 1 were applicable the eutectic would have to be composed of $\gamma^{\prime}+\gamma$ phases and such a eutectic microstructure was not observed. Additional experiments at lower rates are are planned to characterize the eutectic structure of this system.

The values of the peritectic and eutectic temperatures and compositions on the diagram of Fig. 13 are compared to the previous work in Table 1. The findings that the Al composition at the eutectic is higher than the peritectic and that the eutectic microstructure is composed of the $\beta$ and $\gamma^{\prime}$ phases agree with the two recent studies [6,7]. The liquidus temperatures on Fig. 13 were taken as the dendrite tip temperatures, determined by taking the eutectic temperature as $1380^{\circ} \mathrm{C}$ and calculating the $\Delta \mathrm{T}$ to the dendrite tip from the length of the dendrites listed in Table 3 using the temperature gradient in the dendrite array zone as the average between the solid and liquid, $3.2^{\circ} \mathrm{C} / \mathrm{mm}$. The eutectic composition of this study was found from the extrapolations of the liquidus lines to the dendrite base temperature and the value so obtained, $24.5 \mathrm{at} \% \mathrm{Al}$, is midway between the two recent studies. Both the $\beta$ and the $\gamma$ dendrite tips were observed to be nonfaceting and so it is likely that the undercooling at the two types of dendrite tips is very small for the relatively low growth rate used here. Therefore, it is likely that little error is introduced in the evaluation of the eutectic composition due to the extrapolation.

The temperature of the eutectic point found here, $1380,{ }^{\circ} \mathrm{C}$ is above the value of the two recent studies $[6,7]$ and below the value in the Singleton review [4]. Because the two phases 


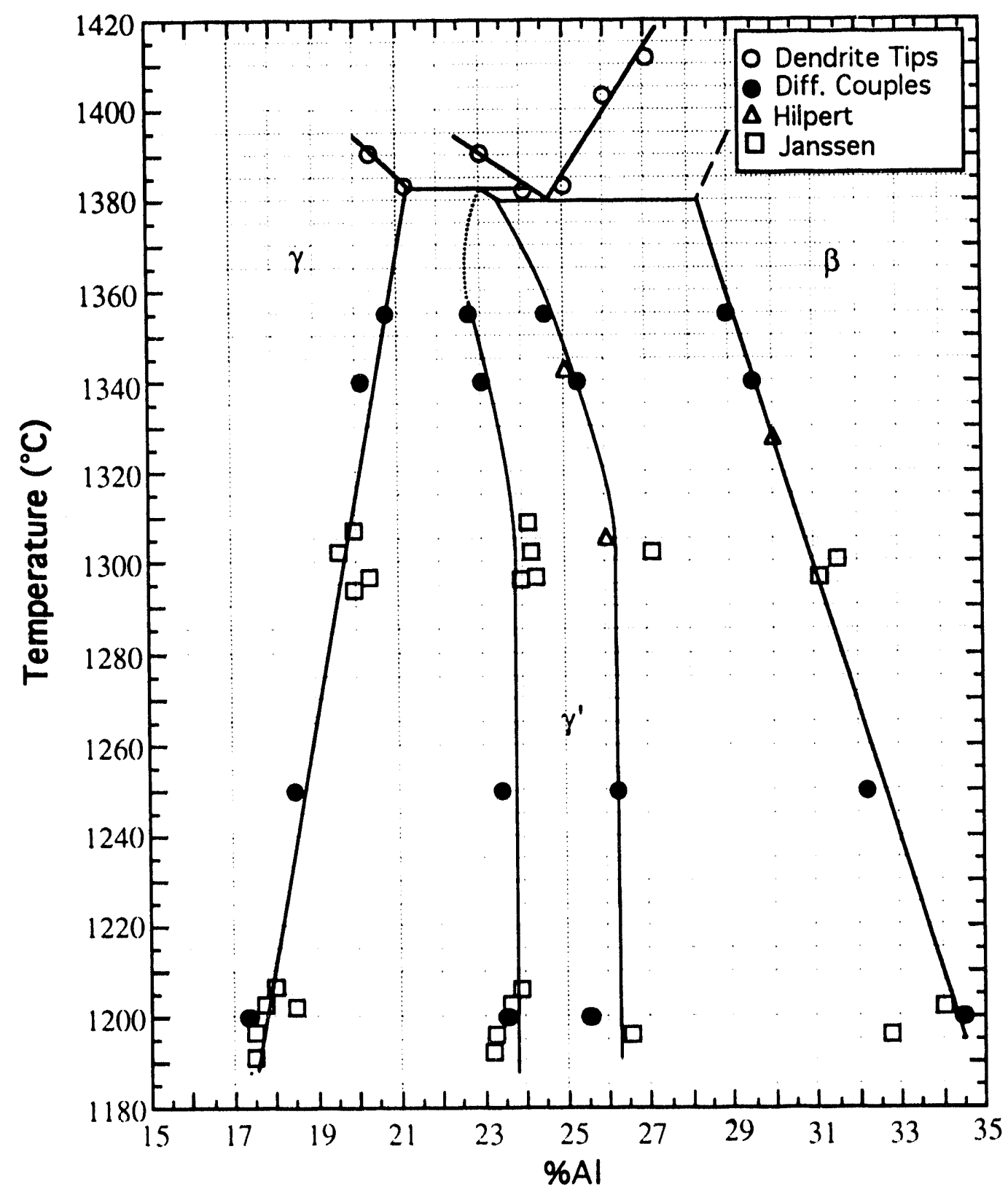

Figure 13. The phase diagram based on the data of this study combined with the solid state data of Hilpert $|6|$ and Jannsen $|8|$ 
which form the eutectic are both nonfaceting and the eutectic has a regular lamellar form, the undercooling at the eutectic can be estimated to be on the same order or less than the $\mathrm{Al}_{-} \mathrm{Al}{ }_{2} \mathrm{Cu}$ system which gives an undercooling of $1^{\circ} \mathrm{C}$ or less at the solidification rate of $61 \mu \mathrm{m} / \mathrm{s}[10]$. Therefore, the undercooling effect is too small to account for the difference found in Table 1. The source of the differences is unknown and further experiments will be required to more accurately evaluate the eutectic temperature.

The slopes of the liquidus lines, $m_{\beta}$ and $m_{\gamma}$, shown on Fig. 13 are compared to the literature values in Table 6. Similar to the eutectic temperatures, it is seen that the data for

Table 6. Data on the slopes of the $\beta$ liquidus, $m_{\beta}$, and the $\gamma$ liquidus, $m_{\gamma}$

\begin{tabular}{|c|c|c|}
\hline Reference & $\mathrm{m}_{\beta}\left({ }^{\circ} \mathrm{C} / \mathrm{at} \%\right)$ & $\mathrm{m}_{\gamma}\left({ }^{\circ} \mathrm{C} / \mathrm{at} \%\right)$ \\
\hline \hline Singleton et. al. $|4|$ & 14.4 & -4.9 \\
\hline Bremer et. al. $|7|$ & 9.8 & -9.7 \\
\hline Hilpert et. al. $|6|$ & 11.8 & -8.4 \\
\hline This study & 14.0 & -6.5 \\
\hline
\end{tabular}

the present study lie closer to those of the Singleton review [4] than the two recent DTA studies [6,7]. Errors in the slope data for the present study would involve errors in evaluating the compositon of the liquid into which the dendrites were growing, errors in evaluating the $\Delta \mathrm{T}$ between the dendrite tips and the eutectic front, and possible variation in the amount of undercooling at the dendrite tips with composition. Errors in the composition were discussed above. Evaluation of $\Delta T$ requires the use of a temperature gradient, but since the liquidus slope would not be affected by errors in the absolute value of the gradient and because the average gradient is likely to be nearly constant with composition, errors arising from the liquid temperature gradient would be small. Similarly, errors arising from undercooling at the 
dendrite tips would be expected to be small because this undercooling would not vary much with compositon for these constant rate experiments. Consequently, the liquidus slopes from this study given in Table VI should be reasonably accurate and further experiments are needed to evaluate which of the values given in the table are more correct.

The peritectic temperature on Fig. 13 has been taken to be $3{ }^{\circ} \mathrm{C}$ above the eutectic temperature after the work of $[6,7]$. It is seen that this value fits well with a linear extrapolation to the intersection of the $\gamma /\left(\gamma+\gamma^{\prime}\right)$ phase boundary determined from the diffusion couple data and the $\gamma$ solidus boundary determined from the tip compositions of the $\gamma$ dendrites. The composition of the $\gamma$ phase at the end of the eutectic horizontal was determined from the experiment on the 24 at $\% \mathrm{Al}$ alloy shown in Fig. 8. At the liquid-eutectic- $\gamma$ trijunction, the eutectic and the $\gamma^{\prime}$ phase are growing at the same temperature. As mentioned above this temperature is expected to lie within $1^{\circ} \mathrm{C}$ of the equilibrium eutectic temperature and it is being used as the eutectic temperature on Fig. 13. The composition of the $\gamma^{\prime}$ phase near the trijunction should correspond to the $\gamma^{\prime}$ composition at the end of the eutectic horizontal. The value of 23.4 at $\% \mathrm{Al}$ used on Fig. 13 was determined from EPMA studies of two large $\gamma$ ' cells similar to the one shown on the right of Fig. 8. The composition was measured in the $\gamma^{\prime}$ cells along a trace colinear with the eutectic growth front. A value of $23.4 \pm 0.22$ at $\% \mathrm{Al}$ was found from repeated evaluations near the eutectic edge.

The peritectic composition shown on Fig. 13 was estimated by assuming that the distribution coefficient, $k$, between liquid and $\gamma^{\prime}$ phases found for the eutectic temperature remains constant as the temperature increases from the eutectic to the peritectic temperatures. The peritectic composition is given as,

$$
\left.X_{p}=k \mid X_{c}-\frac{\Delta T}{m}\right)
$$

where $\mathrm{X}_{\mathrm{c}}$ is the eutectic composition of $24.5 \mathrm{at} \% \mathrm{Al}, \mathrm{m}$ is the liquidus slope and $\Delta \mathrm{T}$ is the difference between the peritectic and eutectic temperatures. Taking $k$ as $23.4 / 24.5, \Delta \mathrm{T}$ as $3{ }^{\circ} \mathrm{C}$ and $\mathrm{m}$ as $-6.5^{\circ} \mathrm{C} / \mathrm{at} \%$, the value of $\mathrm{X}_{\mathrm{p}}$ is found to be 23.0 at $\% \mathrm{Al}$. 
The micrograph of Fig. 8 shows that the $\gamma^{\prime}$ phase is growing slightly ahead of the eutectic front at the centerline of the $\gamma$ region on the right of the photo. Taking the temperature gradient in the dendrite array region as $3.2^{\circ} \mathrm{C} / \mathrm{mm}$ this lead distance corresponds to only 0.06 ${ }^{\circ} \mathrm{C}$. If the undercoolings at the eutectic and the $\gamma$ centerline were the same, this would indicate that the value of $\Delta \mathrm{T}$ between eutectic and peritectic temperatures was less than $0.1^{\circ} \mathrm{C}$. As indicated above the undercooling at the eutectic is probably less than $1^{\circ} \mathrm{C}$. However the solutal undercooling at the $\gamma$ centerline could be large and one cannot, therefore, estimate $\Delta \mathrm{T}$ from the data of Fig.8. Consequently, these experiments were unable to evaluate $\Delta \mathrm{T}$ and the value of 3 ${ }^{\circ} \mathrm{C}$ was taken from the previous experiments $[6,7]$.

The diagram of Fig. 13 shows the Ni composition of the $\gamma^{\prime}$ phase dropping significantly as the temperature decreases below the peritectic value. There are two pieces of information in these experiments that support this result. (a) Figure 7 shows that the eutectic structure has converted to $100 \% \gamma^{\prime}$ at a distance of $4.4 \mathrm{~mm}$ below the eutectic growth front and the temperature profile of Fig. 12 shows that this distance corresponds to a temperature drop of $19^{\circ} \mathrm{C}$. The $\left(\beta+\gamma^{\prime}\right) / \gamma^{\prime}$ phase boundary at the eutectic composition on Fig. 13 is located $20^{\circ} \mathrm{C}$ below the eutectic point. (b) The $\gamma^{\prime}$ phase of the eutectic microstructure is shown in Fig. 9 to have formed a $\gamma$ precipitate by solid state diffusion during the quench. This means that the composition of the $\gamma^{\prime}$ component of the eutectic must lie above the lower temperature $\gamma^{\prime} /\left(\gamma^{\prime}+\gamma\right)$ phase boundary, which is consistent with Fig. 13.

The data of Fig. 10 indicate a $\beta$ composition in the eutectic lying well above the value of 28.2 at\% Al predicted by the diagram of Fig. 13. This was an unexpected result because it was thought that the quench would have been able to freeze-in the eutectic composition forming at the growth front. Janssen $|8|$ has evaluated the mutual diffusion coefficient in the $\beta$ phase from his diffusion couple data and the results give a value ranging from $8.5 \times 10^{-8}$ to $1.1 \times 10^{-8}$ $\mathrm{cm}^{2} / \mathrm{sec}$ for temperatures of 1380 and $12(0){ }^{\circ} \mathrm{C}$. Taking the mean diffusion distance as $\sqrt{\mathrm{D}}$, the time required for the atoms to move $1 \mu \mathrm{m}$ for a temperature drop from 1380 to $12(0)^{\circ} \mathrm{C}$ is 
somewhere between 0.3 and $0.9 \mathrm{sec}$. The value of $1 \mu \mathrm{m}$ is on the order of the original $\beta$ plate thickness calculated from the phase diagram and the observed eutectic spacing. A time of 0.3 to $0.9 \mathrm{sec}$ to cool the growth front from 1380 to $1200^{\circ} \mathrm{C}$ after dropping the sample inside of the $1.5 \mathrm{~mm}$ wall $\mathrm{Al}_{2} \mathrm{O}_{3}$ tube into stagnant water is reasonable. Therefore, it seems likely that the $\beta$ phase of the eutectic has changed its composition from the predicted value of 28.2 at $\% \mathrm{Al}$ at the eutectic growth front to the values of around 34 at $\%$ Al found in the samples after the quench. Solid state diffusion in this system is relatively fast. The composition of the $\gamma^{\prime}$ phase in the eutectic has also changed during the quench. The original composition at the growth front is expected to lie somewhere between the two upper curves on Fig. 10 and this is consistent with the value of 23.4 at\% Al used on the phase diagram of Fig. 13.

The diagram of Fig. 13 has been drawn using the same liquidus slope for the $\gamma$ phase, $m \gamma$, and the $\gamma^{\prime}$ phase, $m_{\gamma^{\prime}}$. Theory requires $m_{\gamma}>m_{\gamma}$, but the present data is not adequate to evaluate the difference in the two slopes. Because of this uncertainty in $\mathrm{m}_{\gamma}$, as well as uncertainties in $\Delta \mathrm{T}$ and the possible effects of solidification rate, future directional solidification experiments are planned at lower solidification rates to better establish the compositions of the peritectic and eutectic reactions. 


\section{CONCLUSIONS}

1. The phase boundaries between the $\beta, \gamma^{\prime}$, and $\gamma$ phases at temperatures just below the freezing range have been determined with diffusion couple experiments and shown to agree well with previous studies $[6,8]$.

2. Directional solidification studies present conclusive evidence that at a solidification rate of $61 \mu \mathrm{m} / \mathrm{s}$ the solidification reactions follow the phase diagram of Fig. 13, which displays a eutectic between the $\beta$ and $\gamma^{\prime}$ phases and a peritectic composition lying at lower $\mathrm{Al}$ concentrations than the eutectic composition. The solid state phase boundaries evaluated at temperatures just below the freezing range are consistent with a lower $\mathrm{Al}$ concentration for the peritectic versus the eutectic compositions. This diagram is similar to the Schramm diagram [5] which is also supported by two recent studies $|6,7|$ but was rejected in the review of Singleton

141. Additional studies at lower growth rates are needed to better establish the composition and temperatures of the peritectic and eutectic reactions. 


\section{REFERENCES}

1. Stoloff, N. S. Int. Met. Rev. 1989, 34, 153.

2. Hansen, M.; Anderko, K. "Constitution of Binary Alloys," McGraw Hill, New York, N.Y., 1958, 118.

3. Metals Handbook, Vol 8, 8th Edition, ASM International, Metals Park, OH, 1973, 262.

4. Singleton, M. F.; Murray, J. L. ; Nash, P. in "Binary Alloy Phase Diagrams," ASM International, Metals Park, OH, 1986, 140.

5. Schramm, J. Z. Metallk. 1941, 33, 347.

6. Hilpert, K.; Kobertz, D.; Venugopal, V.; Miller, M.; Gerads, H.; Bremer, F. J.; Nickel, H. Z. Naturforsch.,1987, 42a, 1327.

7. Bremer, F. J.; Beyss, M.; Karthaus, E.; Hellwig, A.; Schober,T.; Welter, J. M.; Wenzl, H. J. Crys. Growth, 1988, 87, 185.

8. Janssen, M. M. P. Met. Trans., 1973, 4, 1623.

9. Au, Y.; Wayman, C. Scripta Met., 1972, 6, 1209.

10. Magnin, P.; Trivedi, R. K. Acta Met., 1990, 39(4), 469. 
PAPER II. ETCHING CHARACTERISTICS OF Ni3Al 


\section{INTRODUCTION}

In the course of a study on the directional solidification of $\mathrm{Ni}-\mathrm{Al}$ alloys having compositions near $\mathrm{Ni}_{3} \mathrm{Al}$ we encountered some unusual etching characteristics which made phase identification difficult. Figure 1 illustrates the phenomenon with a longitudinal section from a directionally solidified $23 \mathrm{a} / \mathrm{Al}$ alloy. The solidification direction was horizontal from right to left and the original primary $\gamma$ dendrites (the fcc Ni phase) have etched dark. The surrounding interdendritic matrix consists of $\gamma+\gamma^{\prime}$, where $\gamma^{\prime}$ is the $\mathrm{Ni}_{3} \mathrm{Al}$ phase, which has etched light. The dark stringers within the light $\gamma^{\prime}$ phase are stringers of $\gamma$ phase. Notice that three regions of a uniformly white etching microconstituent appears at the longitudinal center of the micrograph. After considerable microprobe work it was concluded that this microconstituent must also be mainly $\gamma^{\prime}$, which probably has a different crystallographic orientation from the $\gamma^{\prime}$ in the surrounding matrix, thereby causing it to etch nearly white. The etch used for Fig. 1 was a modified Marbles etch $\left(10 \mathrm{~g} \mathrm{CuSO}_{4}, 50 \mathrm{ml} \mathrm{HCl}, 50 \mathrm{ml}\right.$ water and $50 \mathrm{ml}$ methanol). The same type of gray level variation was also found with an alternate etch (7.5 $\mathrm{ml} \mathrm{HF}, 2.5 \mathrm{ml} \mathrm{HNO}_{3}$, and $200 \mathrm{ml}$ methanol). It was concluded that perhaps the $\gamma$ phase displays a strong variations in gray level after etching depending upon crystallographic orientation. A systematic study using selective area channelling patterns (SACP) in the scanning electron microscope (SEM) has revealed such a correlation of the intensity of the etched surface appearance versus crystallographic orientation and the results are presented here. 


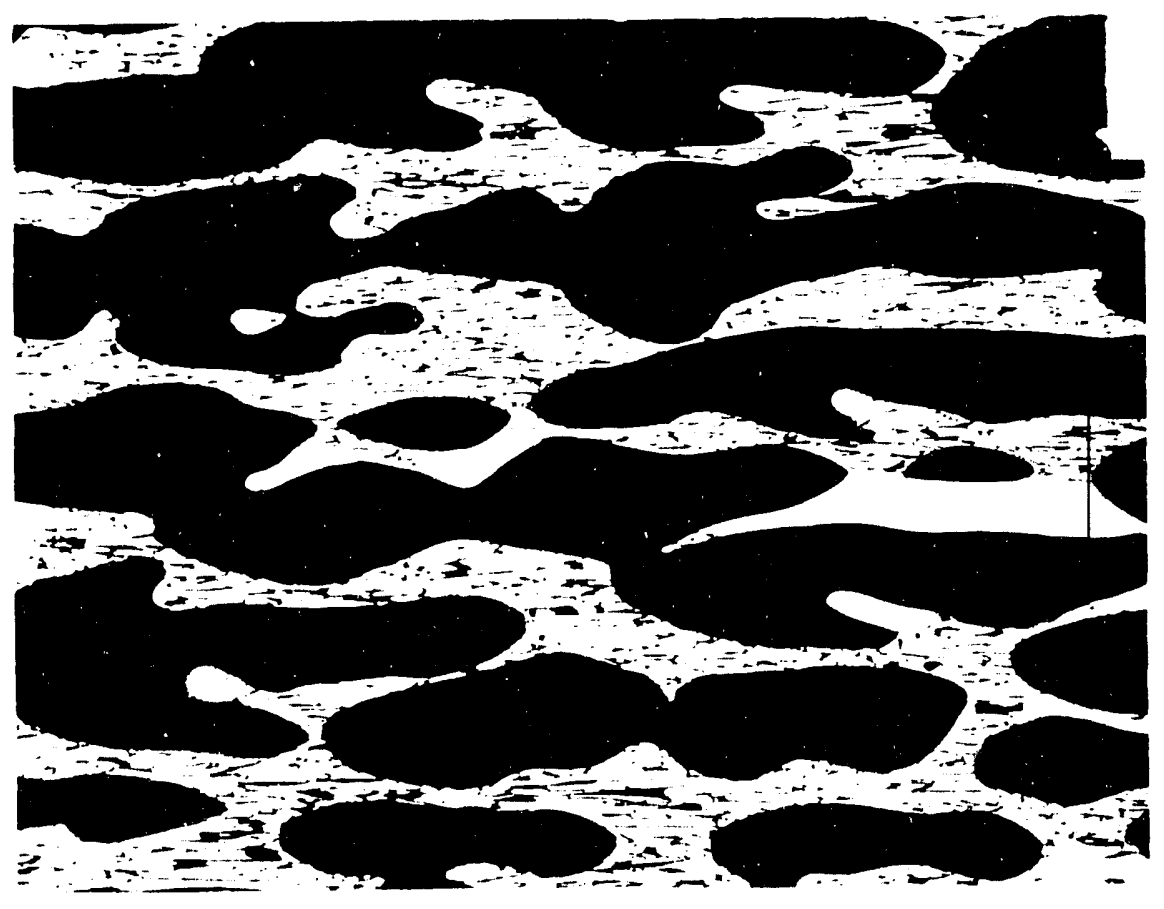

Figure 1. A longitudinal section of a directionally solidified $23 \% \mathrm{Al}$ alloy etched with modified Marbles eich. Solidification rate $=3.2 \mu \mathrm{m} / \mathrm{s}$. Original mag. $=50 \mathrm{x}$ 


\section{EXPERIMENTS}

A polycrystalline rod of $\mathrm{Ni}_{3} \mathrm{Al}$ was prepared with standard metallographic techniques consisting of polishing on $\mathrm{SiC}$ wheels, electropolishing and etching with the modified Marbles etch. Electropolishing was required to improve the quality of the channelling patterns. It was found that the appearance of the various grains ranged from a dull white to fairly dark with intermediate gray levels. Figure 2 presents a typical micrograph from this sample illustrating what was termed a dark grain (a), a gray grain (b) and a white grain (c). After evaluating the gray levels of the various grains by optical microscopy samples were examined in an SEM that employed the deflection-focusing technique [1] for producing SACPs. The instrument had been modified with a radially modulated objective lens current applied to a spiral scan to correct for spherical aberration. This modification provided selected area channelling patterns from regions as small as $2 \mu \mathrm{m}$ diameter. Channelling maps of the standard triangle were first prepared to assist in identifying the orientations revealed by the channelling patterns from the various grains. Maps prepared from single crystal $\mathrm{Ni}$ and large grained $\mathrm{Ni}_{3} \mathrm{Al}$ were indistinquishably different. Figure 3a presents a typical SACP from a grain having an orientation very close to $\langle 111\rangle$. The corresponding crystallographic orientation of this channelling pattern is shown on the stereographic projection at the bottom of the figure. 


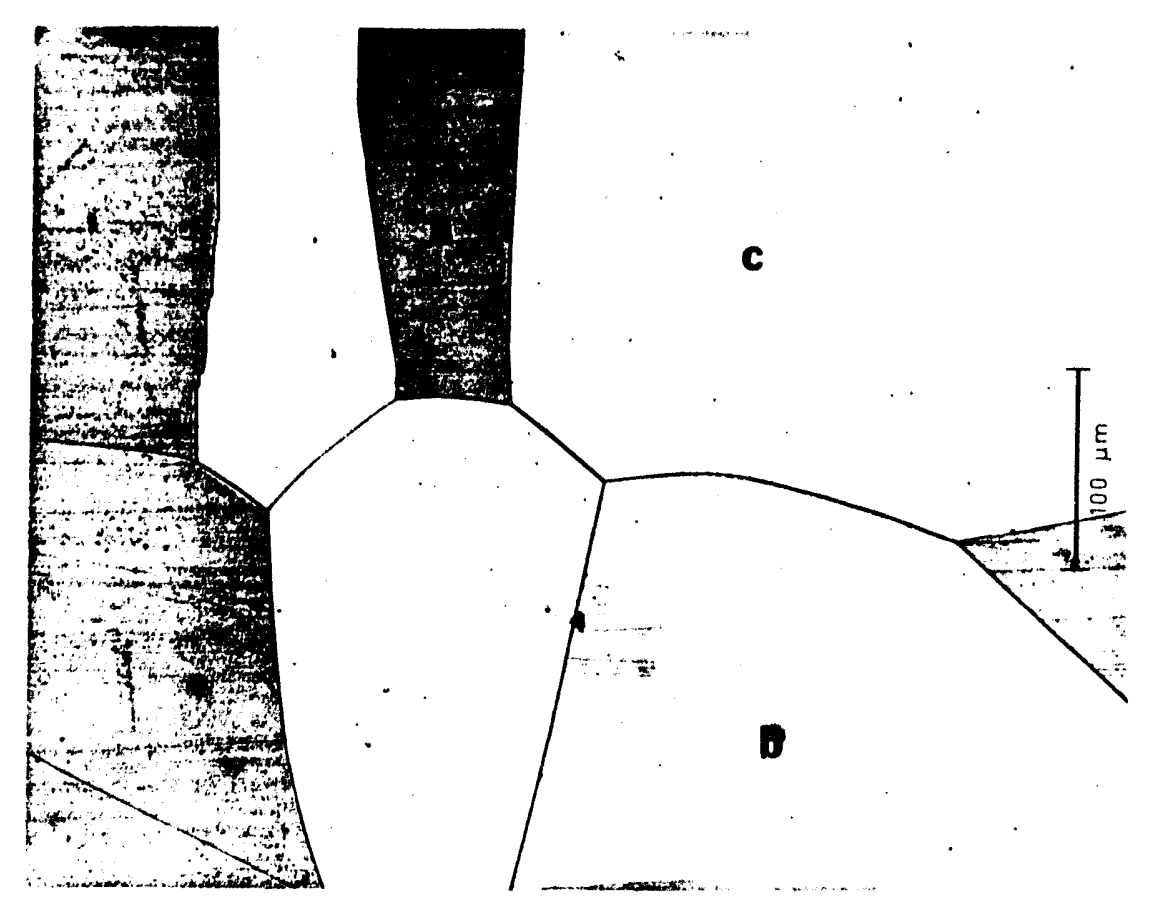

Figure 2. A polycrystalline $25 \%$ Al alloy etched with modified Marbles etch. All grains are $\gamma^{\prime}$ phase. Original mag. $=200 \mathrm{x}$ 
a)
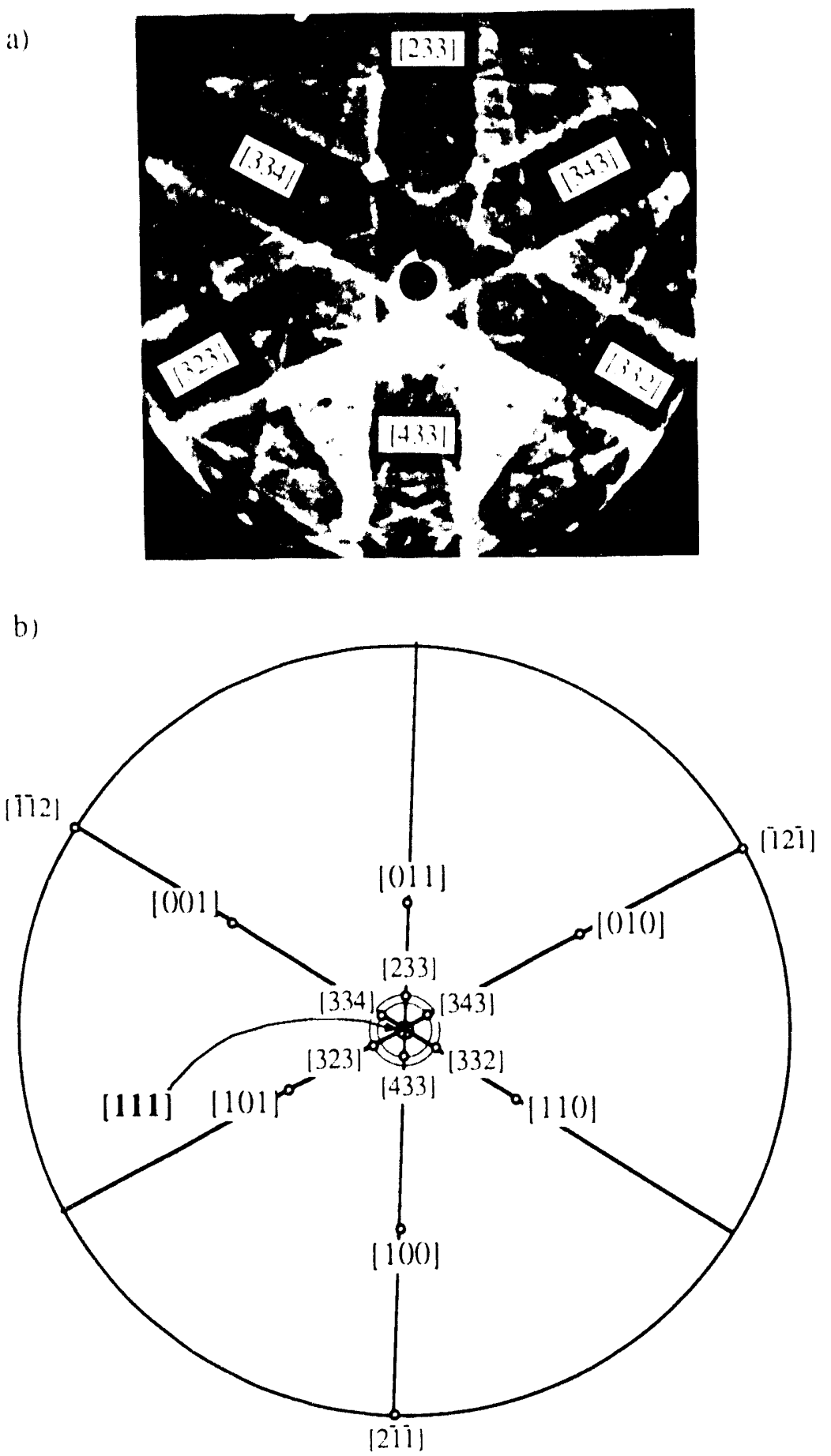

Figure 3. (a) A selected area channelling pattern (SACP) from a grain oriented very close to a $<111>$ direction (Grain A on Fig.4). (b) The bottom figure shows a stereographic projection locating the $<1(x)>,<11()>,<233>$ and $<334>$ directions corresponding to the orientation of the upper SACP 


\section{RESULTS AND DISCUSSION}

\section{Etching Contrast in Single Phase $\gamma^{\prime}$ Alloys}

A total of 105 grains where classified by optical microscopy into the three grey levels (dark, gray and white) and the orientation of the grains were determined with the SACP technique. The results are shown with the square symbols on the standard triangle plot of Fig. 4. A strong correlation of gray level with orientation is revealed, with the dark grains clustering near the $<111>$ orientation and the white grains near the $<001>$ orientation. Dashed lines have been drawn on Fig 4 to delineate the transitional orientations to the gray shaded grains. The dark grains appear at orientations that vary from around 30 to 35 degrees of $<111>$ while the white grains appear at orientations varying from around 13 to 32 degrees from $<(0) 1>$. The white microconstituent on Fig. 1 is a region of the $y^{\prime}$ matrix surrounding the dark dendrites that has recrystallized giving an orientation near $\langle(0) 1\rangle$.

In an attempt to understand the mechanism producing the observed gray level variation with crystallographic orientation, grains at the three gray levels were examined at higher magnifications in the SEM, and the results are shown on Fig. 5. Note that the magnification in these photos is fairly large, $13000 \mathrm{x}$, and the corresponding surface features are small, about at the limit of the optical microscope, $\approx 0.2 \mu \mathrm{m}$. It is seen that the surface roughness increases going from $<()() 1>$ to $<114>$ to $<111>$ orientations and this change would account for the increase in the gray level (darkening) observed optically for this orientation change. The rougher surface would more effectively scatter light away from the observer and produce a darker appearance.

Close examination of Fig. 5a suggests that the $\{111\}$ surface might be covered with 3 sided pyramids. The stereographic projection of Fig. 3 shows that a 1111 direction has three $\{100\}$ planes, $((0) 1),(010)$ and $(100)$ arranged symmetrically around it such that these three planes could form the surface of the pyramids. To further investigate this possibility grains 


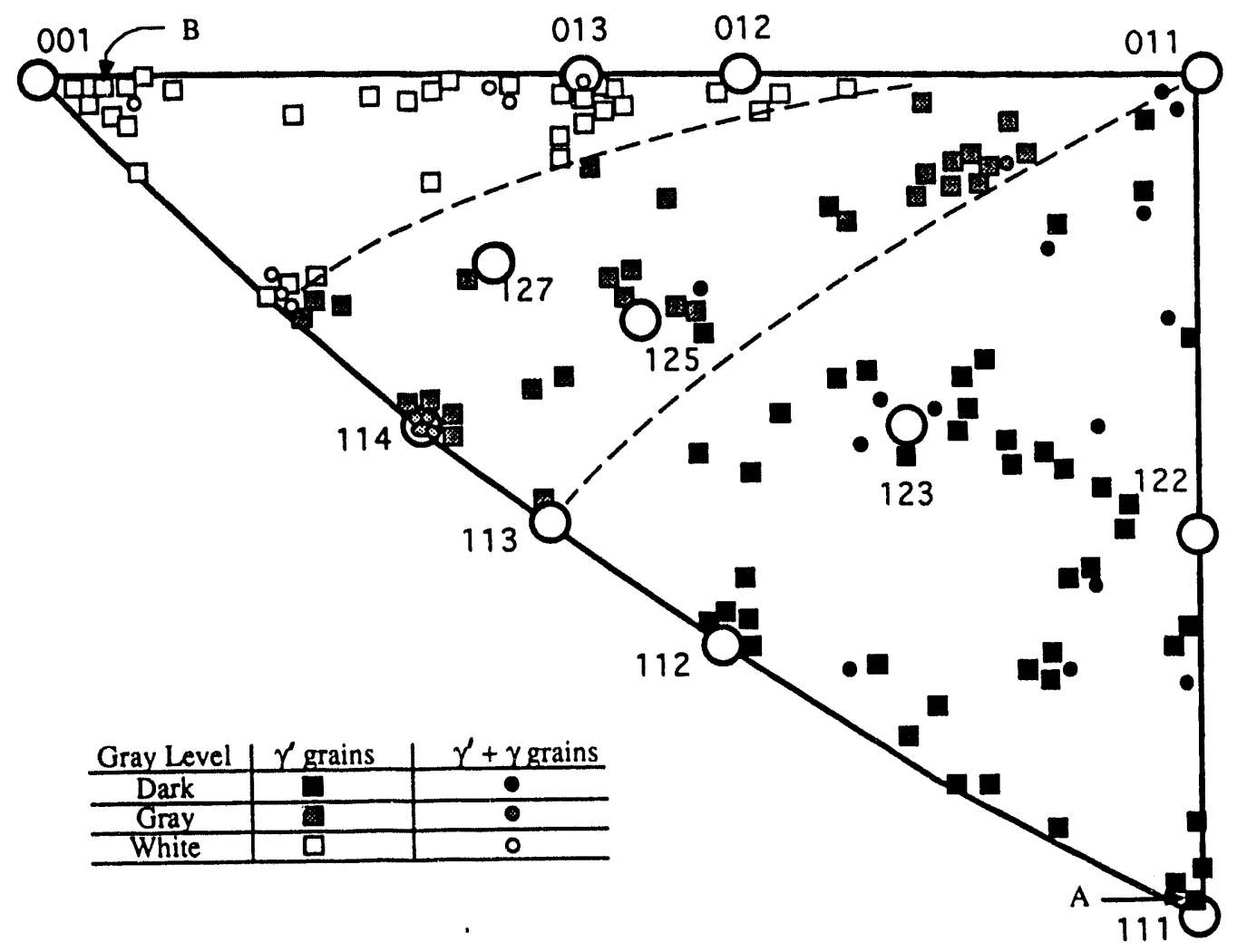

Figure 4. The crystallographic orientation of various $\gamma^{\prime}$ and $\gamma+\gamma^{\prime}$ grains determined by SACP evaluation after their gray levels had been evaluated by optical microscopy 


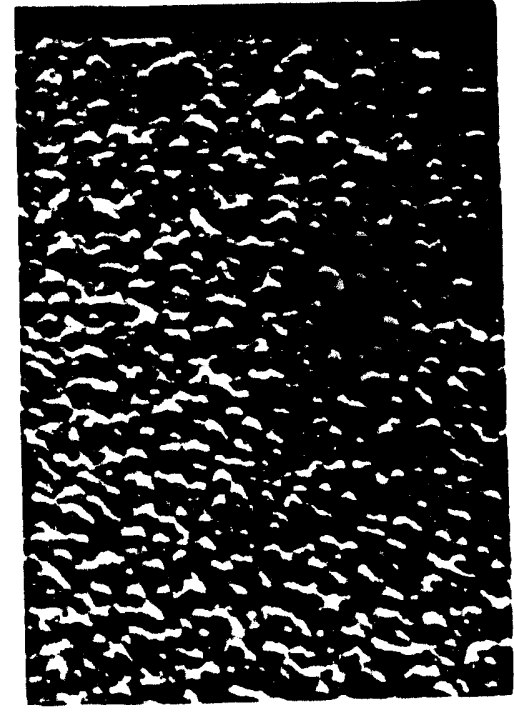

(a)

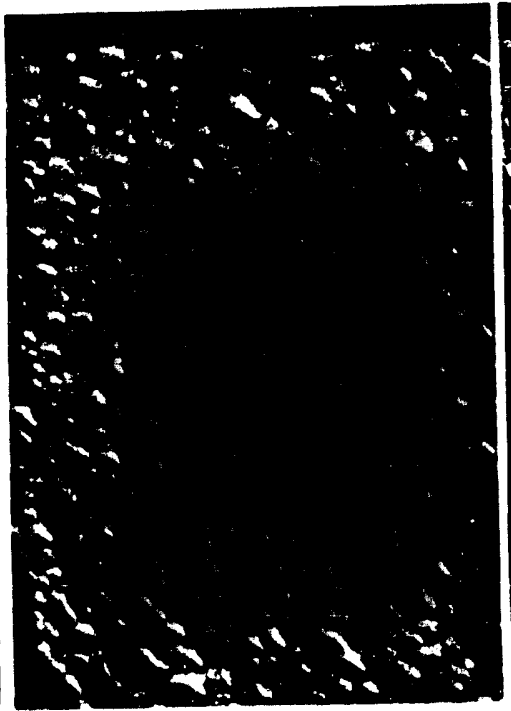

(b)

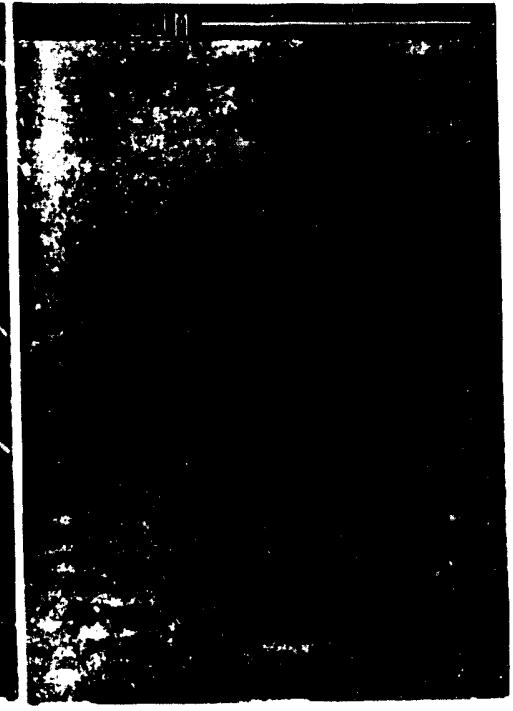

(c)

Figure 5. High magnification SEM pictures of etched surfaces having the orientations: (a) $\langle 111\rangle$, (b) $<114>$ and (c) $<001>$. Original mag. $=11,300 x$ 
oriented near $<111\rangle$ were deep etched and Fig. $6 \mathrm{~b}$ shows a high magnification $(21,000 \mathrm{x})$ image of one of the larger typical surface features, which can be interpreted to have the pyramidal geometry shown in Fig. 6a with the three pyramid planes consisting of $\{100\}$ planes having steps on them as shown. The $\{001\}$ planes do not appear to be perfectly flat, but have small protrusions upon them. These small protrusions have sizes around 90 to $100 \mathrm{~nm}$ and they are probably also pyramidally shaped, but the resolution of the SEM available was inadequate to resolve them well enough to confirm this possibility.

Experiments were carried out to verify that the crystallography of the etched grains was consistent with the pyramid planes being $(100)$ planes as opposed to some other plane set displaying the 3 -fold symmetry, such as $\{110\}$. Fig. 7a shows a near $<111>$ oriented grain on the left labelled $A$, and a near $<(0) 1>$ oriented grain on the right, labelled $B$. The orientations of these two grains are identified on Fig. 4. Examination of this grain pair as well as several others has revealed that the near $<111>$ oriented grains are etched away much faster than the near $<10()>$ grains. This fact turns out to be important in understanding the etching contrast of the grains containing $\gamma+\gamma^{\prime}$ phases, which will be discussed later

Figure $7 \mathrm{~b}$ is a high magnification SEM micrograph of the $<111>$ oriented grain $A$ at the location labelled $7 \mathrm{~b}$ on Fig. 7a. Several 3-sided pyramid-like features are apparent. In order to verify that the faces of these pyramids could be $\{100\}$ planes it was necessary to determine the crystallographic orientation of grain A relative to the three edges of the pyramid features. This was done using the grain boundaries labelled I and II on Fig. $7 \mathrm{a}$ as reference lines. There are problems involving rotation shifts between the SACP and the surface image $[2,3]$ and the use of non co-linear reference lines is useful in eliminating such rotations. The SACP from grain A is shown in Fig. 3. By changing the sample height without adjusting the lens currents Fig. 7c was obtained in which the grain boundaries I and II appear along with a reduced, but nonrotated channelling pattern. (It was necessary to take these patterns, Figs 3 and 7c, prior to the deep etching because the deep etching severely distorts the patterns.) From Figs $7 c$ and 3 it is 
(a)

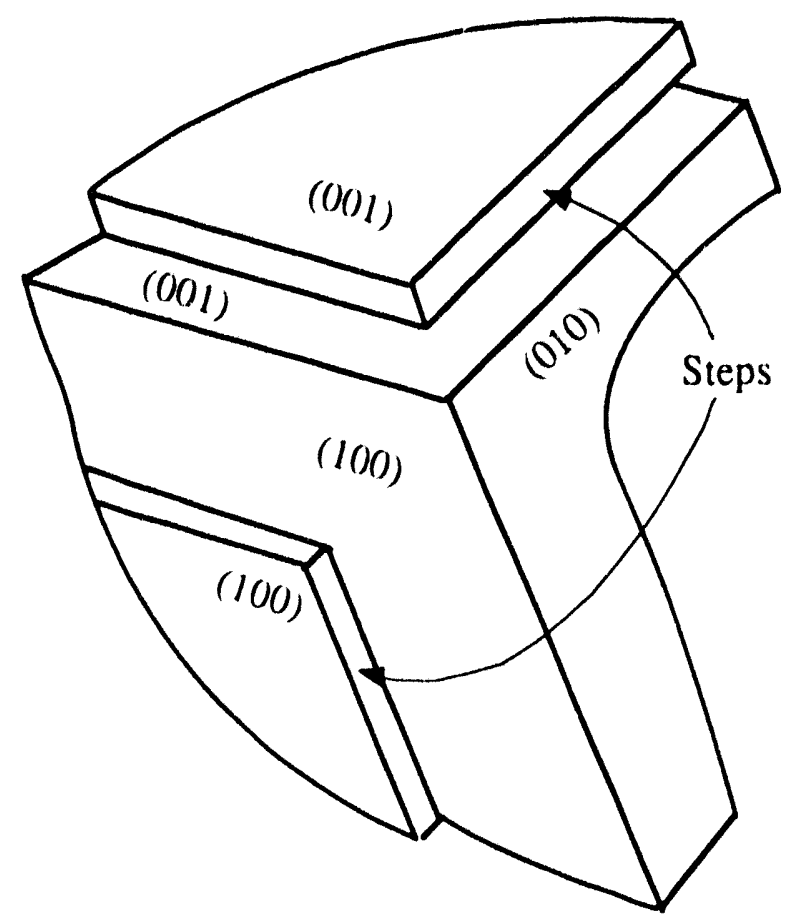

(b)

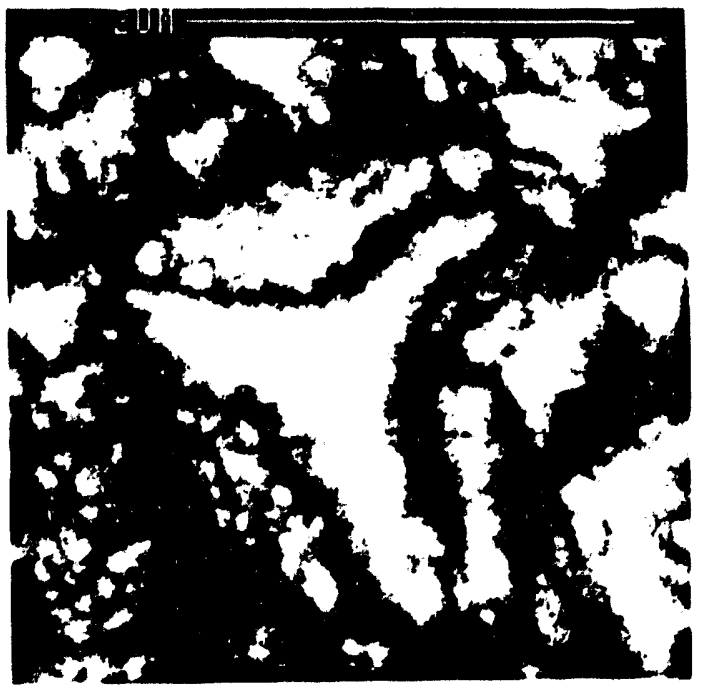

Figure 6. A deep etched (30 sec, Marbles etch) surface of a near $\langle 111\rangle$ grain with a pyramid structure at the center is shown in (b) and a sketch of the proposed planes making up the surface of the pyramid is shown at (a) 
(a)

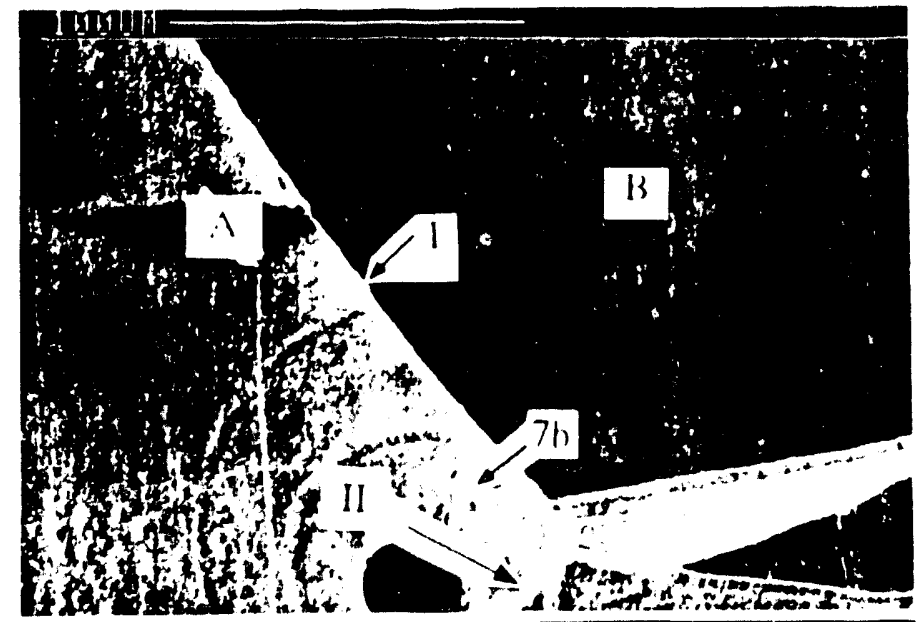

(b)

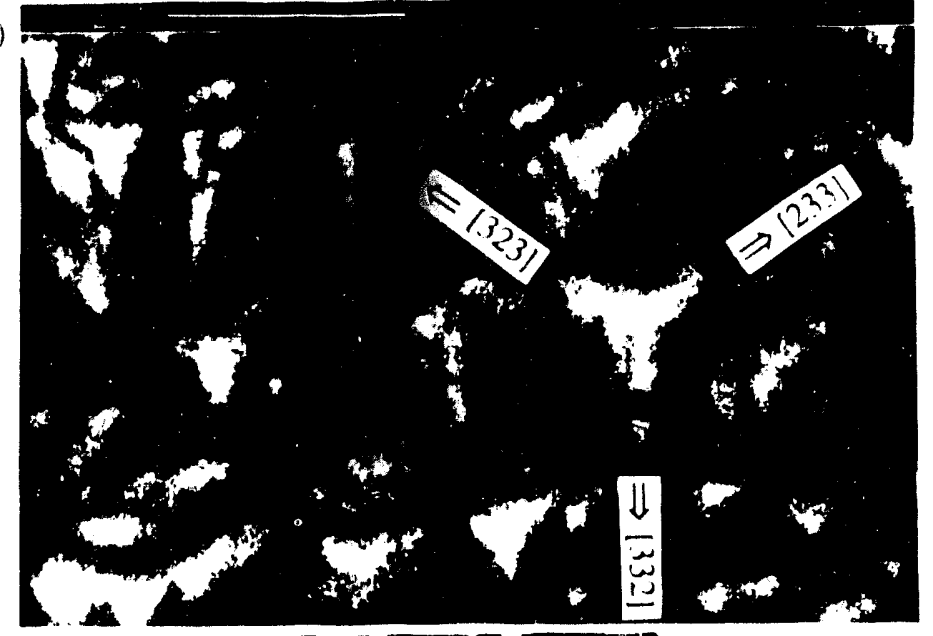

(c)

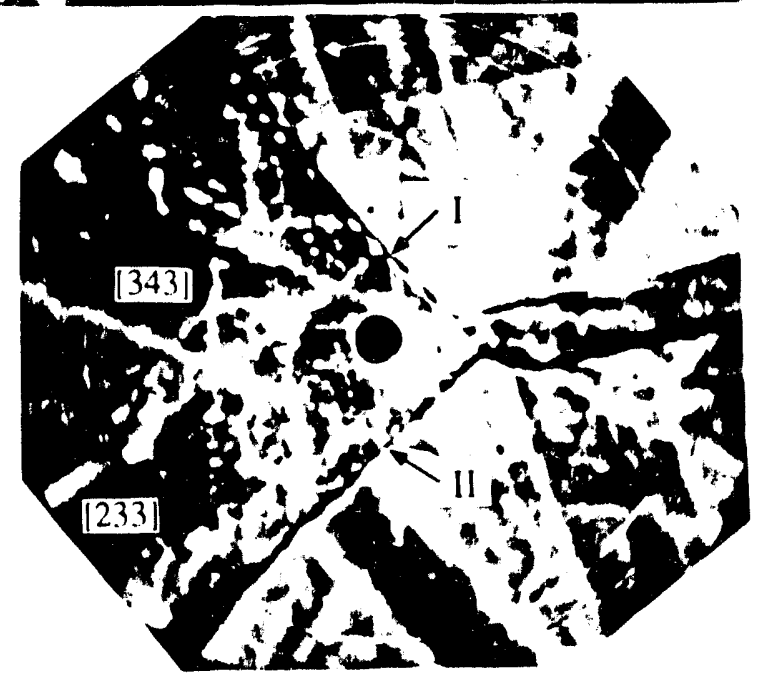

Figure 7. (a) A deep etched surface showing a $<111>$ oriented grain at $A$ and a near $\langle()() 1\rangle$ grain at $B$ and the grain boundary I separating the two grains. Original mag. = 33()x. (b) A high magnification picture of grain $A$ at the point labelled $7 \mathrm{~b}$ on Fig. 7a. Original mag. $=11()()() x$. (c) An SACP of grains $A$ and $B$ with sample height changed to reveal both the channelling pattern and surface topography 
possible to evaluate the location of the reference boundaries I and II on the SACP. Figures 7a and $7 \mathrm{~b}$ are not rotated with respect to each other so that from these two figures it is possible to locate the alignment of Fig. $7 \mathrm{~b}$ with respect to boundaries I and II and, hence with respect to the SACP of Fig. 3. It is very important here to know that the channeling pattern appears in a 180 degree rotation relative to the sample image [3]. From these measurements it was determined that the three edges of the pyramids shown by arrows on Fig. $7 \mathrm{~b}$ lie parallel to the $<233>$ directions, as indicated on Fig. $7 \mathrm{~b}$. Comparison to the stereographic projection of Fig 3 shows that the three $<001>$ directions lie opposite the three $<233>$ directions labelled on Fig. 7b. This result shows that the orientation relationship between the ridges of the pyramids and the crystallography of the grains is consistent with the pyramid face planes being $\{100\}$ planes. Another grain pair was examined and the same result as shown on Fig. 7 was confirmed.

\section{Etching Contrast in $\gamma+\gamma^{\prime}$ Two Phase Alloys}

Alloys which contained $27 \mathrm{a} / \mathrm{Al}$ formed primary $\gamma$ phase dendrites. Upon cooling to room temperature the $\gamma^{\prime}$ phase precipitated in these dendrites to produce a two phase $\gamma+\gamma^{\prime}$ structure with a high volume fraction of $\gamma^{\prime}$ phase. It is well established that the $\gamma^{\prime}$ phase forms a coherent precipitate with matching cube faces and one expects that the $\gamma^{\prime}$ phase may be crystallographically aligned throughout each single crystal dendrite. All of the dendrites in Fig. 1 (dark shaded microconstituent) were found to have the same crystallographic orientation throughout with SACP, which is consistent with this idea.

Figure 8 presents a transverse section of a solidified rod which shows primary dendrite stalks that had grown in a direction toward the observer contained in a matrix consisting of $\gamma^{\prime}$ (white) with thin dark filamentary $\gamma$ phase subdividing it into a cellular arrangement. Upon cooling some of the dendrites have recrystallized, thereby producing the very dramatic changes in gray levels seen on Fig. 8. The micrograph clearly illustrates that the two phase $\gamma+\gamma^{\prime}$ 


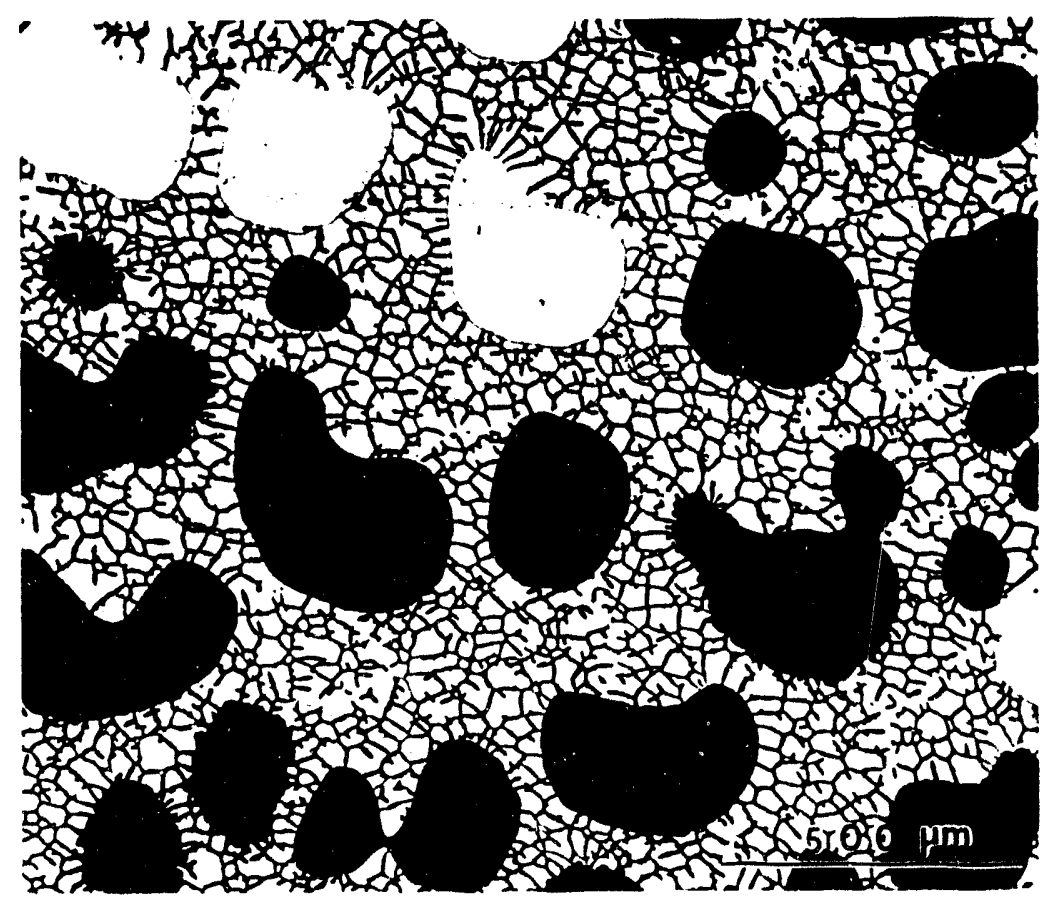

Figure 8. A transverse section showing primary $\gamma$ dendrites formed in a $\gamma+\gamma$ matrix. An alloy of $23 \% \mathrm{ol}$ at a growth rate of $1.5 \mu \mathrm{m} / \mathrm{s}$. Original mag. $=50 \mathrm{x}$ 
dendrites may have various shades of gray. SACP analysis shows that the white dendrites have maintained an orientation near their $<001>$ growth direction while the darkest dendrites have recrystallized to orientations near $\langle 111\rangle$. A systematic study of the relationship between gray level and crystallographic orientation was carried out on $26 \gamma+\gamma^{\prime}$ dendrites and the results are presented by the circles on Fig. 4. It is seen that the same correlation is found as for the single phase $\gamma$ grains, orientations near $<111>$ are dark and a transition to light occurs as $<001>$ is approached. It should be noted, however, that the gray level of the dark dendrites is considerably blacker than that of the dark single phase $\gamma^{\prime}$ grains.

A high magnification SEM micrograph of a dark dendrite is shown in Fig. 9a and that of a white dendrite in Fig. 9b. The light colored phase on these micrographs is the $\gamma$ phase, which occurs as a minor volume fraction for the $23 \mathrm{a} / \mathrm{Al}$ composition. It does not etch as rapidly as the $\gamma^{\prime}$ phase and therefore protrudes above the surface and emits a larger number of secondary electrons in SEM examination because of its increased surface to volume ratio, thus producing the white gray level on the SEM micrographs. Comparison of these two micrographs reveals that the $\gamma^{\prime}$ phase etches much faster relative to the $\gamma$ phase in the dark near $<111>$ oriented dendrites. This result is consistent with the fact observed above that $<111\rangle$ oriented $\gamma^{\prime}$ grains etch considerably faster than $<001>$ oriented $\gamma^{\prime}$ grains. The surface of Fig. 9a would be more effective in scattering light than that of Fig. $9 \mathrm{~b}$ and would thereby produce the darker gray levels observed for the $<111>$ orientations in optical micrographs. In addition to this mechanism of light scattering, the $\gamma^{\prime}$ fraction of the surface would also be more effective in scattering light by the same mechanism as observed for the single phase $\gamma^{\prime}$ grains. The fact that the dark grains of the two phase $\gamma+\gamma^{\prime}$ dendrites possess a significantly blacker gray level than the dark grains of the single phase $\gamma$ grains (compare the dark dendrites of Fig. 1 to the dark $\gamma^{\prime}$ grains of Fig. 2) is apparently due to the presence of these two mechanisms for producing enhanced surface roughness in the two phase dendrites examined here. It seems likely that this enhanced dark gray level in the near $<111>$ oriented two phase $\gamma+\gamma^{\prime}$ alloys 


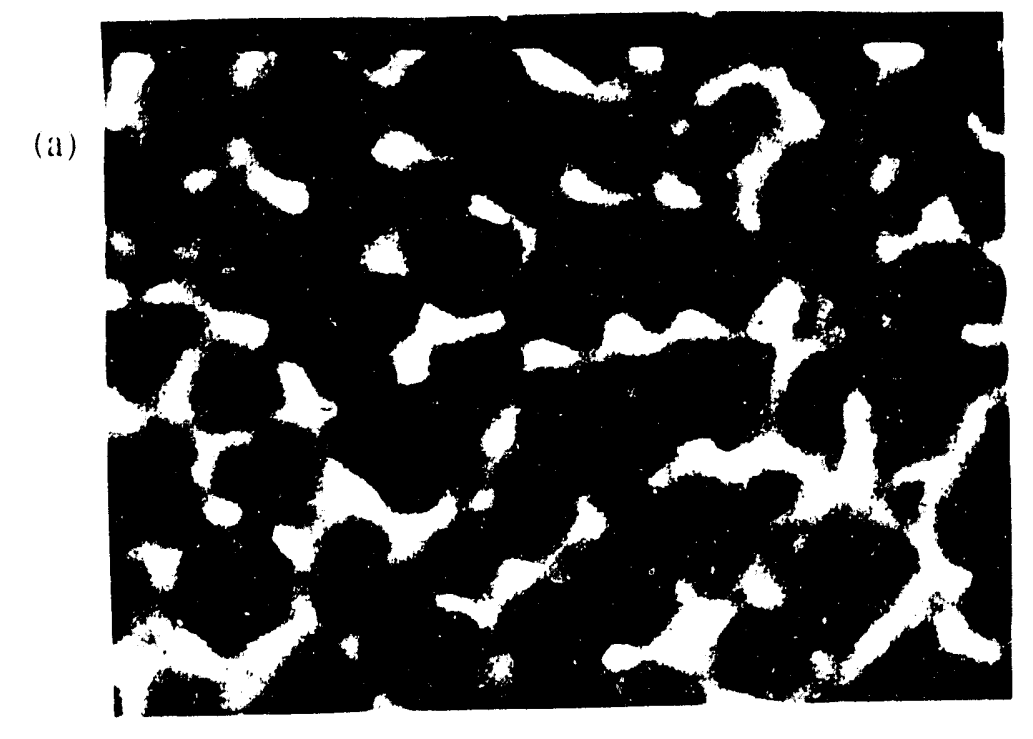

(b)

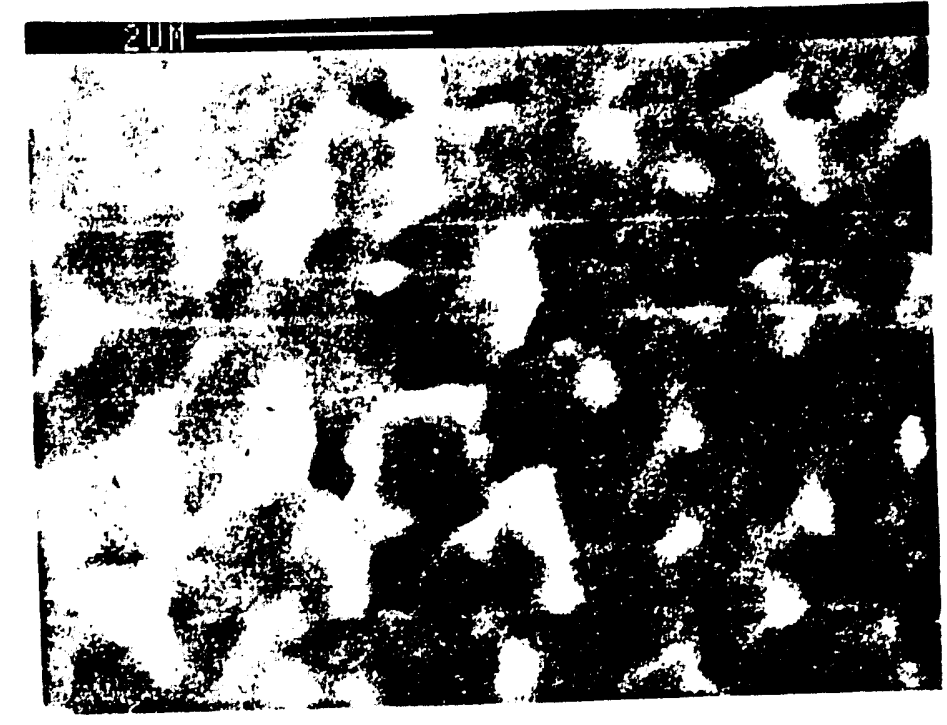

Figure 9. SEM micrographs of etched (a) dark dendrite and (b) light dendrite. Original mag. = $11,3()() \mathrm{x}$ 
would depend upon the size of the $\gamma^{\prime}$ precipitate phase and, hence, may depend fairly strongly upon the prior thermal history. Our results have found that the variation in gray level from $<111>$ to $<001>$ is less in alloys solidified more rapidly, and this result is apparently due to the finer $\gamma^{\prime}$ precipitate size. 


\section{CONCLUSIONS}

1. A significant variation in gray level is observed in single phase $\gamma^{\prime}$ grains after etching. It has been shown with aid of the SACP technique in an SEM that a strong correlation exists with gray level and crystallographic orientation. Grains oriented within roughly 30 to 35 degrees of a $<111>$ direction appear dark while grains oriented within roughly 13 to 32 degrees of a $<001>$ direction appear white. Intermediate orientations display intermediate gray levels.

2. The etchants attack the $\{111\}$ surfaces much faster than $[001\}$ surfaces. On $\{111\}$ surfaces pyramidal protrusions appear and the orientation relationship between the edges of the pyramids and the crystallographic directions of the grains is consistent with the faces of the pyramids being $\{001\}$ planes.

3. It seems likely that the etchants are unable to remove atoms from $\{001\}$ faces as rapidly as from faces of other orientations as this would account for: (a) the slower etch rate of \{001\} faces, (b) the formation of \{001\} faces on the pyramidal structures of etched (111\} surfaces and (c) the absence of surface features on the $\{001\}$ faces. Based on this result one would expect the $\{100\}$ faces to possess a lower surface energy. A literature search has found a theoretical calculation of the cohesive energies of $\mathrm{Ni}_{3} \mathrm{Al}$ grain boundaries [4] which shows the $\{111\}$ boundaries with a lower energy than the $\{001\}$ boundaries. The apparent lower energy of $\{001\}$ surfaces predicted by the results of this study is perhaps due to an oxide formation modifying the chemistry of the surfaces formed on etching.

4. Primary $\gamma$ dendrites in a $23 \mathrm{a} / \mathrm{Al}$ alloy decomposed into two phase $\gamma+\gamma^{\prime}$ structures with the $\gamma^{\prime}$ precipitates maintaining the same crystallographic orientation as the parent $\gamma$ phase. Upon etching, these two phase dendrites produced the same variation in gray level with 
crystallographic orientation as found for the single phase $\gamma^{\prime}$ grains. The variation in gray level was larger than for the single phase case and this was shown to result from a differential etch rate between the two phases which increases for orientations near $\langle 111\rangle$. 


\section{REFERENCES}

1. Joy, D. C.; Newbury, D. E.; Davidson, D. L. J. Appl. Phys., 1982, 53, R81.

2. Van Essen, C. G.; Verhoeven, J. D. J. Phys. E, 1974, 7, 768.

3. Verhoeven, J. D.; Gibson, E. D. J. Phys. E, 1975, 8, 15.

4. Chen, S. P.; Voter, A. F.; Albers, R. C., Boring, A. M.; Hay, P. J. J. Mater. Res., $1990,5,955$. 
PAPER III. THE $\gamma^{\prime} / \gamma^{\prime}$ INTERFACES INDUCED BY FORMATION OF THE $\gamma^{\prime}$ BAND AT THE SOLID-LIQUID INTERFACE DURING QUENCH PROCEDURE 


\section{INTRODUCTION}

In the directional solidification of $\mathrm{Ni}-\mathrm{Al}$ alloys planar interfaces of $\gamma$ or $\gamma$ could be obtained at low rates in the hypo-eutectic alloys of $\mathrm{Al} 23,23.5$, and 24 at.\% (these alloys will be designated $\mathrm{Al} 23, \mathrm{Al} 23.5$, and $\mathrm{Al} 24$, respectively). It is a very predictable result at low rates because the planar stability is mainly controlled by the $\mathrm{G} / \mathrm{V}$ values. The $\gamma$ planar interface was changed to $y$ planar at high solidification fractions in these alloy compositions because convection caused composition changes along the directionally solidified rod. At normal solidification fractions ( $\mathrm{fs}=0.45$ ), the $\gamma$ planar interface was found in the Al 23 alloy and the $\gamma$ planar interface was found in alloys Al 23.5 and Al 24. The initial phase was always the $\gamma$ planar at low rates in these hypo-eutectic compositions. Actually the $\gamma$ planar interface was not all $\gamma$ planar at normal solidification fraction in Al 23 alloy. The edge part of the $\gamma$ planar in the directionally solidified rod was the $\gamma+y^{\prime}$ coupled structure.

An interesting result was found at the solid-liquid interface position. The usual quenching process always produced an about $150 \mu \mathrm{m}$ thick $\gamma^{\prime}$ band in front of the planar solidliquid interface. The $\gamma^{\prime}$ band appeared in the Al 23, Al 23.5, and Al 24 alloys at the planar solid interface where the solid consisted of pure $\gamma$ phase, or coupled $\gamma+\gamma$ phases, or a mixture of pure $\gamma$ phase surrounded by coupled $\gamma+\gamma$ phases. Another $\gamma$ band was also found above the $\gamma^{\prime}$ band formed in front of the planar solid-liquid interface. The lower $\gamma^{\prime}$ band and the upper $\gamma^{\prime}$ band are called the $\gamma^{\prime}$ lower band and the $\gamma^{\prime}$ upper band respectively. Two $\gamma^{\prime} / \gamma^{\prime}$ interfaces bordered the $\gamma^{\prime}$ lower band, one at the top and the other at the bottom. The origin of these interfaces was not well understood and these two $\gamma^{\prime} / \gamma^{\prime}$ interfaces have been termed the mystery interface \# I (below the ylower band on solid side of original interface) and \# II (above the $\gamma_{\text {lower }}$ band on liquid side of original interface). These mystery interfaces and the $y$ bands were revealed on etching in Marbles solution, or in HF solution, or with electro-polishing, or with argon ion beam etching. 
An important question was raised here as to which interface, \# I or \# II, is the real solid-liquid interface. It could be thought that the mystery interface \# II was a solid-liquid quenched interface and the mystery interface \# I was a solid state transformation interface in $\mathrm{Al}$ 23 alloy. At first, the mystery interface \# II was thought to be the solid-liquid interface in $\mathrm{Al}$ 24 alloy because the mystery interface \# I was ambiguous in the $\gamma^{\prime}$ planar interface. If the mystery interface \# I were the solid-liquid interface, the interface \# II was really a strange boundary. Several possibilities were suggested for the cause of these mystery interfaces in the $\gamma^{\prime}$ band region : Orientation change, order-disorder transition, or composition variation.

In order to determine where the solid-liquid quenched interface is and understand the origin of these mystery interfaces, several experiments and analyses were done. The role of quench rate on the formation of the $\gamma^{\prime}$ band and mystery interfaces was investigated by changing the quench media. A composition change was searched for in the $\gamma^{\prime}$ band region by EDS (Energy Dispersive Spectroscope) in both the SEM and TEM. The orientation difference and structure change were investigated in TEM with diffraction pictures and in the SEM with SACP (Selected Area electron Channeling Pattern). The solid-liquid boundary region was also observed at high magnification by TEM. A series of heat-treatments was done to observe the transition of these mystery boundaries. 


\section{EXPERIMENTS}

The general directional solidification technique and sample preparation technique were described in Paper I. Usual quenching was done by dropping the container tube holding the solid-liquid interface into a water bucket through the cold finger at termination of the directional solidification. In order to investigate the role of quench rate on the formation of the mystery interfaces, different quench rates were obtained by changing the quench media. The Ga-In eutectic, which is liquid at room temperature, was used for faster quench rates. Ice water was also used instead of water. Slower quench rates could be obtained by quenching either inside the cold finger or to air. The cold finger quench rate is faster than an air quench but slower than a water quench. An additional quench method was developed which did not move the sample during the quenching procedure. The alumina tube below the furnace was put inside the water jacket. An O-ring sealed the water between the alumina tube and water jacket, as shown in Figure 1. Quenching was accomplished without moving the sample by hoisting the furnace using a lever. It is called "up" quench because the furnace and coolant move up during the quench procedure. It could prove whether there is any perturbation during the normal drop quenching procedure. This quench rate was expected to be faster than the normal water quench rate because the quench distance and time were short and water was flowing during quenching.

A selected area electron channeling technique (SACP) was used to investigate the orientations of $\gamma^{\prime}$ and $\gamma$ phases in the solid-liquid interface region. Composition changes were examined in the mystery interface region with EDS in the SEM using chemical analyzed standards for composition calibration. These techniques were described in Parts I and II.

TEM was the most important tool to identify the nature of the interfaces and the $y^{\prime}$ band. A Philips CM-30 TEM was used at $30(0) \mathrm{kV}$. The solid-liquid interface region was examined with high magnification bright and darkfield image patterns. The selected area diffraction patterns and convergent beam diffraction patterns were used to investigate any 


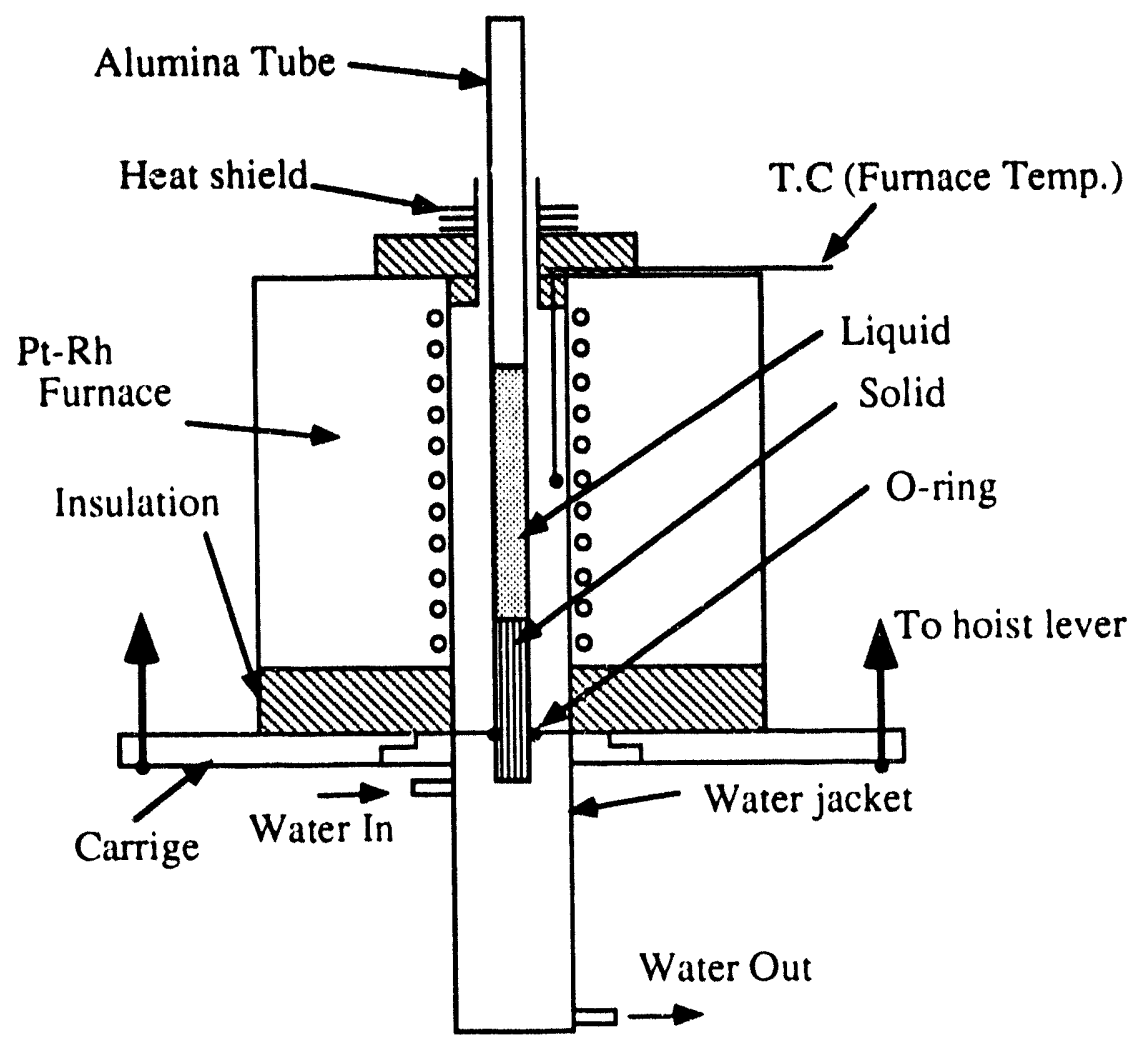

Figure 1. Schematic of up quench arrangement in the directional solidification unit. The cold finger of the normal quench fixture was replaced by the water jacket 
structure or orientation changes below and above the $\gamma^{\prime}$ band. A very local composition changes across the interface \#I and \#II could be examined by EDS techniques in the TEM to reduce the size of the analyzed position. While the analyzed size was $1-2 \mu \mathrm{m}$ in SEM, that was reduced to less than $100 \mathrm{~nm}$ in the TEM. The sample preparation must be careful to make a hole in the $\gamma^{\prime}$ band region. The sample of this interface region was cut and made into a disk ( 3 mm diameter and $0.25 \mathrm{~mm}$ thickness), where the $\gamma^{\prime}$ band region was positioned on the center of this disk. This disk was dimpled 90 $\mathrm{mm}$ on each side in the dimpler, and then jet polished until just before making a hole using the polishing solution $63 \%$ methanol, $35 \% 2$ butoxyethanol, and $2 \%$ perchloric acid at $-45 \mathrm{C}$ and $50 \mathrm{~V}$ conditions. Fortunately it could be seen after jet polishing where the interface region was located. This jet polished disk was then ion milled to make a hole in the $y$ band region. Because the initial hole was not exactly on the region of the $y^{\prime}$ bands, the hole was enlarged to this region. Figure 2 shows how this hole grows to the interface region after 9,18 , and $24 \mathrm{~h}$ ion milling in the y planar interface of Exp. \# 21 (Al 23-().8 $\mu \mathrm{m} / \mathrm{s}$. Note: This sample does not contain an interface \# I. The lower interface is a $\gamma^{\prime} / \gamma$ boundary.). A hole, which passed through the mystery boundary II, $\gamma$ bands, and $\gamma$ planar interface, was obtained, as shown in Figure 2(c). After additional experiments on $\gamma+\gamma^{\prime}$ coupled growth were successful, a foil was produced with a hole through the interfaces \# I and II.

A series of heat-treatments was carried out to observe changes in the mystery interfaces. The sample was sealed in a quartz tube under an argon atmosphere to prevent oxygen contamination. A piece of sample was heat-treated just until the mystery interfaces disappeared, where the orientations were examined with SACP (Selected Area electron Channeling Pattern) before and after heat-treatment to determine when or if recrystallization occurred. 
i)

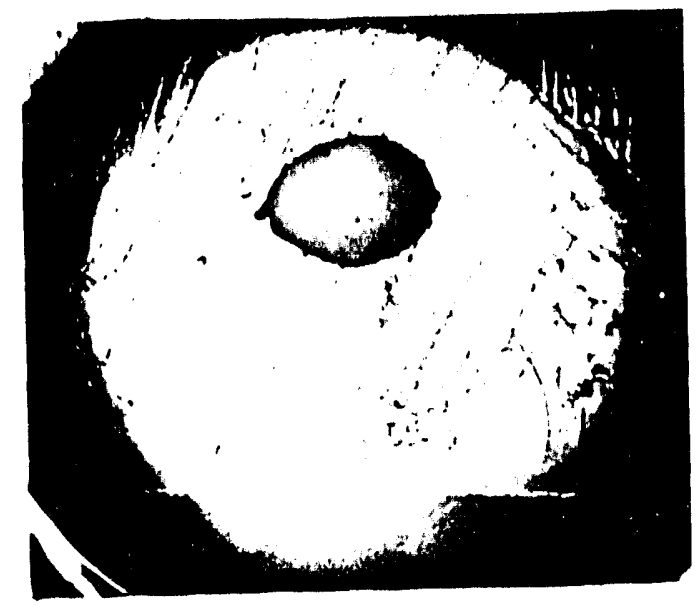

b)

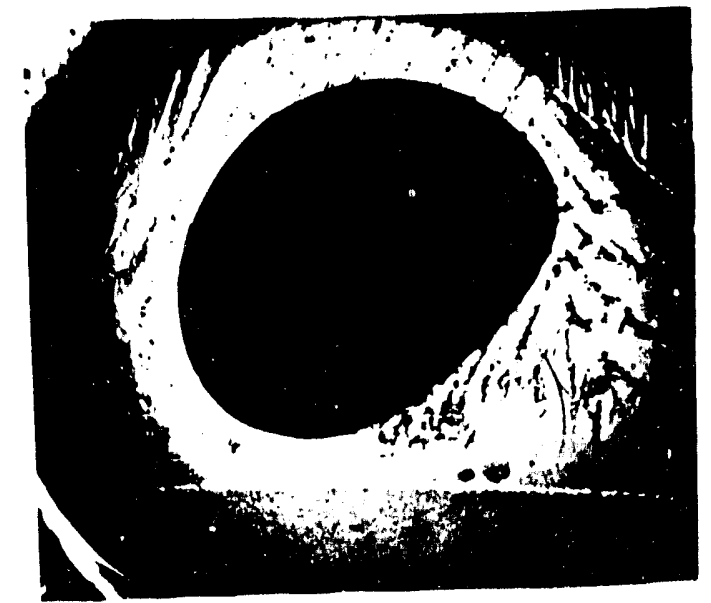

c)

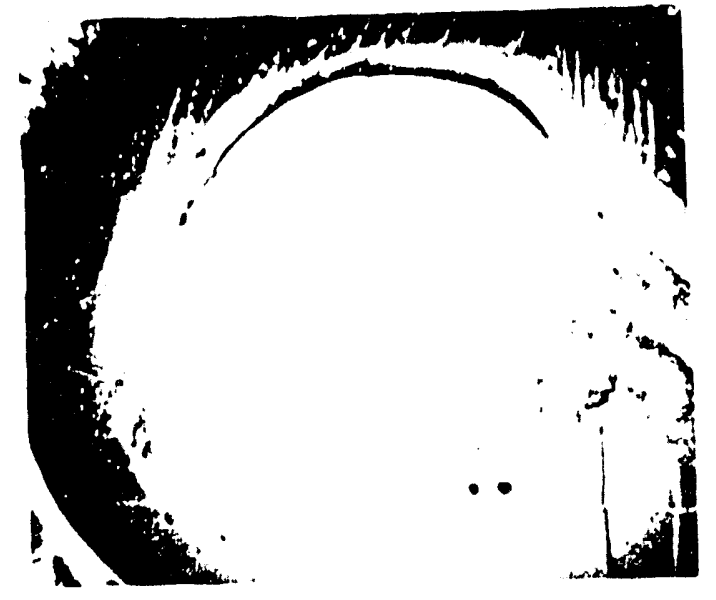

Figure 2. Growing procedure of a hole for TEM. A hole was grown near the solid-liquid interface region after a) $9 \mathrm{~h}$, b) $18 \mathrm{~h}$, and c) $24 \mathrm{~h}$ in the ion miller. 50x 


\section{RESULTS}

\section{Quench Rate Control}

Various quench rates were obtained by changing the quench media. According to the quench rate it was observed how the $\gamma^{\prime}$ bands and mystery interfaces were changed, as shown in Table 1. For each experiment the table lists the solidification rate, the ratio of temperature gradient to growth rate, $\mathrm{G} / \mathrm{V}$, the volume fraction of original liquid that was solidified before quenching, fs, the method of quenching, the type of interface (P means planar, D means dendritic, $\left(\gamma+\gamma^{\prime}\right) \mathrm{P}$ means two phase $\gamma+\gamma^{\prime}$ with planar interface, $\gamma \mathrm{P}\left(\gamma+\gamma^{\prime}\right.$ edge $)$ means a central $\gamma$ planar interface surrounded by coupled $\gamma+\gamma^{\prime}$ phases, $\gamma D+\left(\gamma+\gamma^{\prime}\right)$ means $\gamma$ phase dendrites with two phase $\gamma+\gamma^{\prime}$ between the dendrites).

Upon quenching, at least one $\gamma^{\prime}$ band always formed at the solid-liquid interface region at normal solidification fractions $(\mathrm{f} s \approx 0.45$ ) at low rates in $\mathrm{Al} 23, \mathrm{Al} 23.5$, and $\mathrm{Al} 24$ alloys. According to quench rates, several kinds of interfaces were found. Figure 3 shows the solidliquid interfaces of (a) water quench (Exp.\#21), (b) ice-water quench (Exp\# 50), (c) upquench (Exp.\# 56), (d) Ga-In quench (Exp.\# 51), and (e) air quench (Exp.\# 123). The mystery boundaries are shown in Figure 4(a)(see arrows), which is a high magnification view of the edge part of Figure 3-(a). In water quenched samples like Figure 3(a), there were two single phase, single grain $\gamma^{\prime}$ bands at the solid-liquid interface, as shown schematically in Figure 4(b). The $\gamma^{\prime}$ lower band between the interface \# I and \# II has a thickness of $\delta_{1}$. A distinction at the edge side and center of the rod is made as $\delta_{1 \mathrm{l}}$ and $\delta_{1 \mathrm{c}}$ respectively. The $\gamma_{u p p e r}^{\prime}$ band above interface \# II has thickness $\delta_{2}$. It is defined at the edge side and center of the rod as $\delta_{2 e}$ and $\delta_{2 c}$ respectively. Both interface \# I and \# II are $\gamma^{\prime} / \gamma^{\prime}$ interfaces. The top boundary of the $\gamma_{\text {upper }}^{\prime}$ band is defined as the location where the elongated polycrystalline $\gamma^{\prime}$ arrays appear. At higher quench rates than used with Figure 3(a), see Figure 3(b, c, and d), the eutectic forms at the region just above the $\gamma^{\prime}$ lower band. In these cases there is no $\gamma_{\text {upper }}^{\prime}$ 
Table 1 The mystery interfaces at low rates solidifications with various quench rates in $\mathrm{Al} 23,23.5$, and 24 alloys

\begin{tabular}{|c|c|c|c|c|c|c|c|c|}
\hline Alloy & Exp.\# & $\begin{array}{c}\text { Rate } \\
(\mu \mathrm{m} / \mathrm{s})\end{array}$ & $\begin{array}{c}\mathrm{G} / \mathrm{V} \\
10^{5} \mathrm{Cs} / \mathrm{cm}^{2}\end{array}$ & fs & Q-method & Interface & Mystery I & Mystery II \\
\hline \multirow[t]{16}{*}{ Al 23} & 21 & 0.8 & 3.04 & 0.45 & Water & $\gamma \mathrm{P}\left(\gamma+\gamma^{\prime}\right.$ edge $)$ & Yes & Yes \\
\hline & 22 & 0.8 & 3.04 & 0.35 & Cold-finger & $\gamma \mathrm{P}$ & - & Yes \\
\hline & 124 & 0.8 & 4.95 & 0.27 & Water & $\gamma \mathrm{P}$ & - & Yes \\
\hline & 48 & 0.8 & 3.04 & 0.57 & Ice W & $\gamma^{\prime} \mathrm{P}$ & No & Yes \\
\hline & 91 & 0.8 & 4.95 & 0.55 & Water & $\gamma^{\prime} \mathrm{P}$ & No & Yes \\
\hline & 50 & 1.1 & 2.21 & 0.48 & Ice W & $\gamma \mathrm{P}\left(\gamma+\gamma^{\prime} \mathrm{edge}\right)$ & Yes & No \\
\hline & 63 & 1.3 & 1.87 & 0.40 & Water & $"$ & Yes & Yes \\
\hline & 64 & 1.3 & 1.87 & 0.45 & Water & $"$ & Yes & Yes \\
\hline & 65 & 1.3 & 1.87 & 0.54 & Water & $\gamma^{\prime} \mathrm{P}$ & No & Yes \\
\hline & 51 & 1.3 & 1.87 & 0.48 & Ga-In & $\gamma \mathrm{P}\left(\gamma+\gamma^{\prime}\right.$ edge $)$ & Yes & No \\
\hline & 47 & 1.5 & 1.62 & 0.58 & Water & $\gamma \mathrm{D}+\left(\gamma+\gamma^{\prime}\right)$ & Yes & Yes \\
\hline & 123 & 2.0 & 1.97 & 0.50 & Air & $\left(\gamma+\gamma^{\prime}\right) P$ & Yes & Yes \\
\hline & 94 & 2.0 & 1.97 & 0.52 & Water & $\left(\gamma+\gamma^{\prime}\right) P$ & Yes & Yes \\
\hline & 55 & 2.0 & 1.21 & 0.40 & Up & $\gamma D+\left(\gamma+\gamma^{\prime}\right)$ & Yes & No \\
\hline & 20 & 3.2 & 0.76 & 0.40 & Water & $"$ & Yes & Yes \\
\hline & 104 & 3.2 & 1.22 & 0.46 & Water & $"$ & Yes & Yes \\
\hline \multirow[t]{2}{*}{ Al 23.5} & 114 & 0.8 & 4.95 & 0.66 & Water & $\gamma^{\prime} \mathrm{P}$ & No & Yes \\
\hline & 112 & 3.2 & 1.03 & 0.31 & Water & $\gamma D+\left(\gamma+\gamma^{\prime}\right)$ & Yes & Yes \\
\hline \multirow[t]{5}{*}{$\mathrm{Al} 24$} & 49 & 0.8 & 3.04 & 0.41 & Ice W & $\gamma^{\prime} \mathrm{P}$ & No & Yes \\
\hline & 57 & 1.5 & 1.62 & 0.42 & Up & $\gamma^{\prime} \mathrm{P}$ & No & No \\
\hline & 56 & 3.2 & 0.76 & 0.35 & Up & $(\gamma$ major $+\gamma) P$ & Yes & No \\
\hline & 27 & 3.2 & 0.76 & 0.41 & Water & $"$ & Yes & Yes \\
\hline & 96 & 3.2 & 1.22 & 0.41 & Water & $\gamma^{\prime} \mathrm{P}$ & No & Yes \\
\hline
\end{tabular}

* P means a planar interface and D means a dendritic interface. 

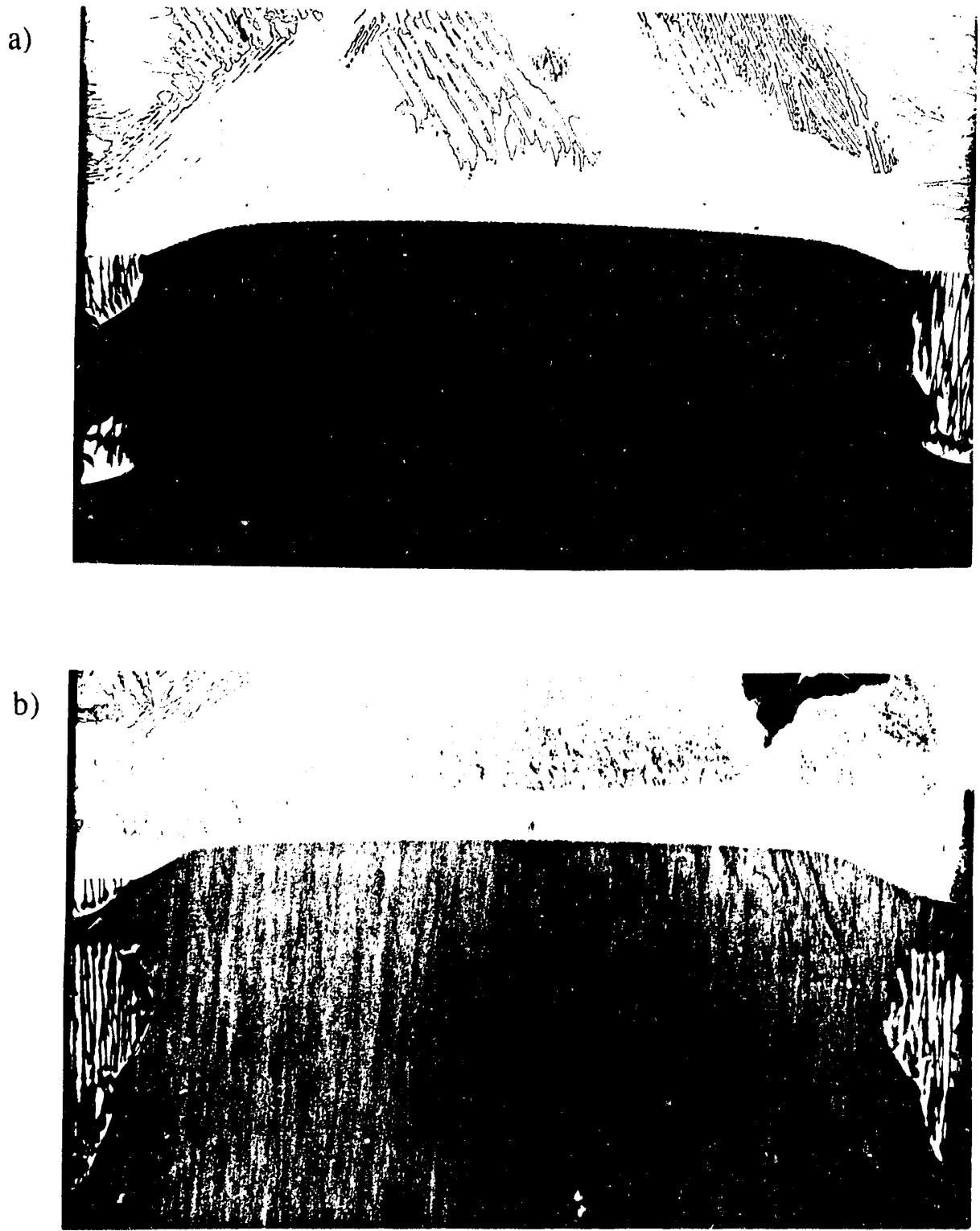

Figure 3. Several kinds of interfaces according to different quench media. 25x,Marbles etch.

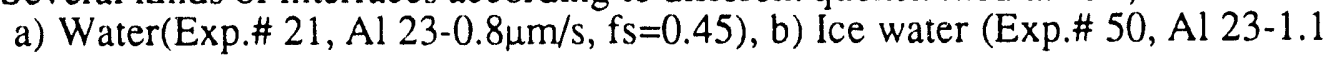
$\mu \mathrm{m} / \mathrm{s}, \mathrm{fs}=0.48), 40 \mathrm{x}$ 
c)
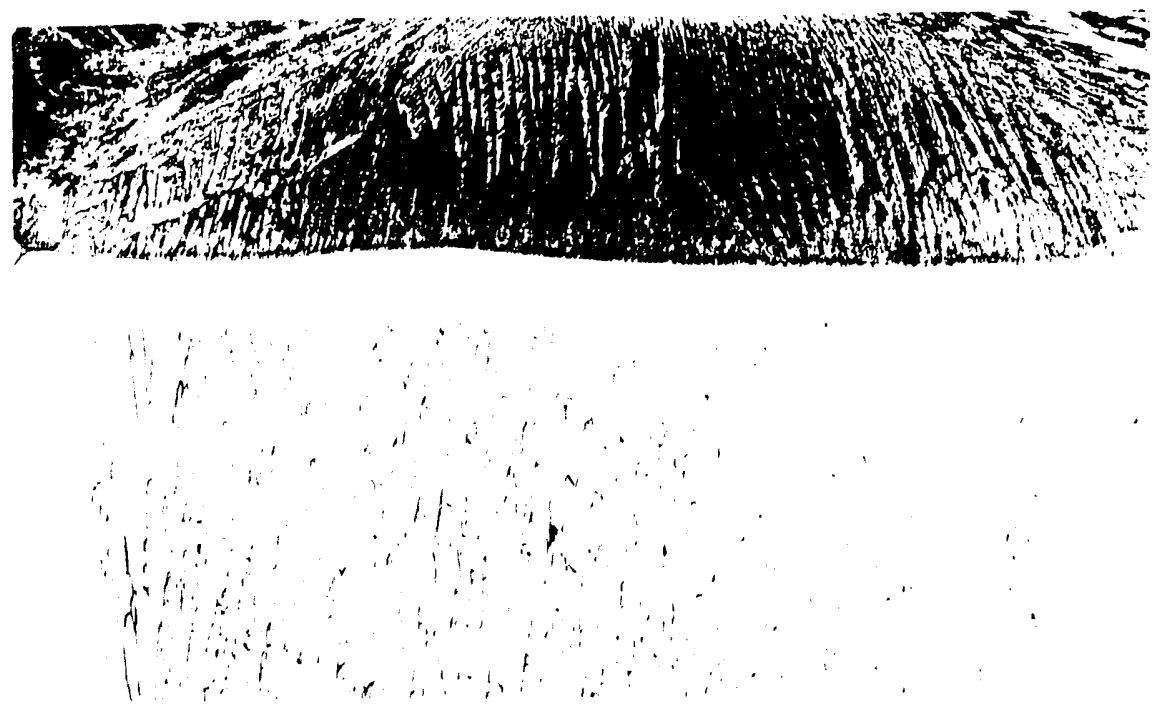

d) $\begin{aligned} & 7 \\ & 7 \\ & 7\end{aligned}$

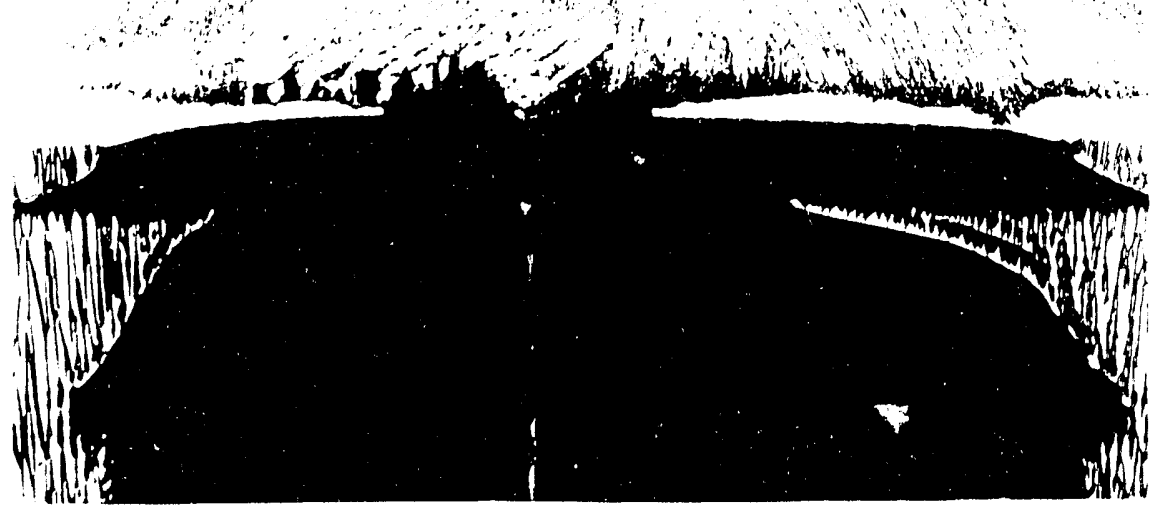

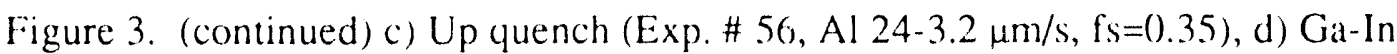

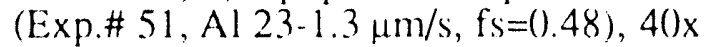




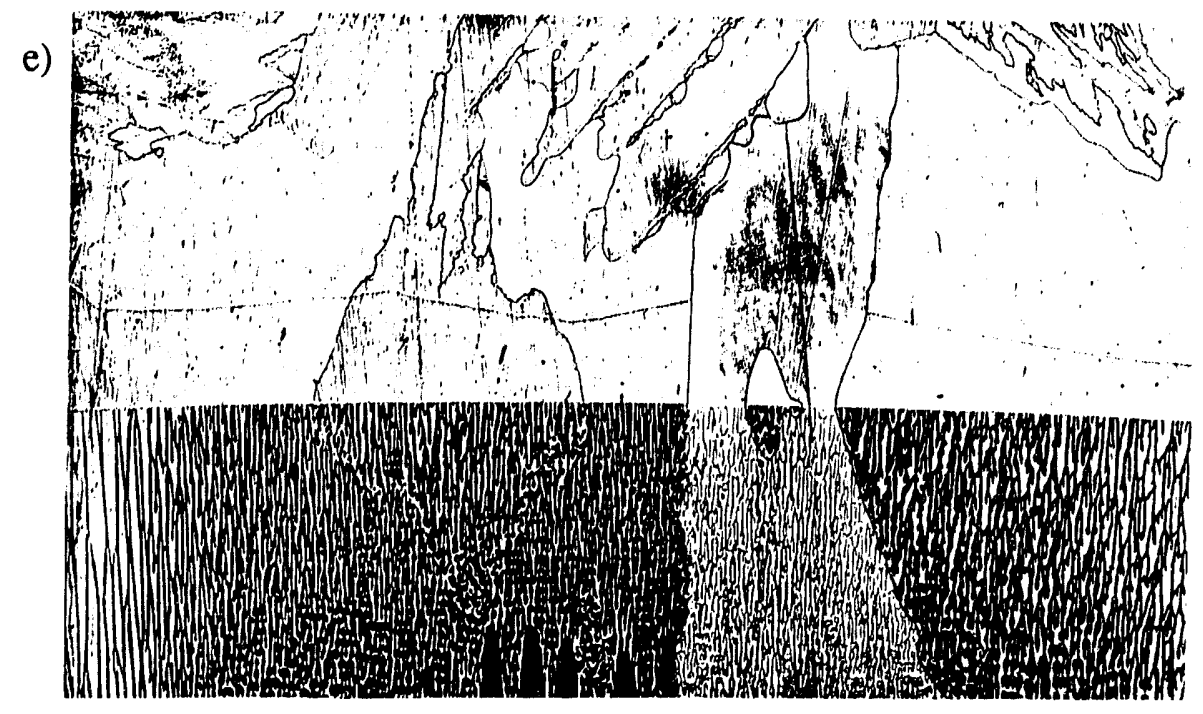

Figure 3. (continued) e) Air (Exp. \# 123, Al 23- $2 \mu \mathrm{m} / \mathrm{s}, \mathrm{fs}=0.50$ ), 40x 
band and the region which had appeared as as a $\gamma_{\text {upper }}^{\prime}$ band at water quench rate is now a eutectic structure with $\beta$ as the second phase. The elongated polycrystalline $\gamma^{\prime}$ arrays meet with the UQL(upper quenched liquid) at the "UQL/Up growing solid" boundary(see Figure 4(b)) where the UQL stands for the quenched liquid lying above advancing solid grown from the solid-liquid interface. The structure of UQL is similar to that of the elongated $\gamma$ polycrystalline region which consists of cellular eutectic sheets at the fast quench of Ga-In.

The $\delta_{1}$ and $\delta_{2}$ values were changed by the quench media, as shown in Table 2 . At normal quench rates of water quenches (up, ice-water, and drop), the $\delta_{1}$ values were similar, however, the $\delta_{2}$ values were decreased following the sequence of water, ice-water, and up quenches. The $\delta_{2}$ values for drop quench were $200-350 \mu \mathrm{m}$ and those for ice-water were 0-80 $\mu \mathrm{m}$. The $\delta_{2}$ values for up quench were essentially zero, where now the upper boundary of the $\gamma^{\prime}$ lower band was no longer a $\gamma^{\prime} / \gamma^{\prime}$ interface, but a $\gamma^{\prime} /$ eutectic interface. This eutectic initially forms as either $\beta+\gamma$ or $\beta+\gamma$. Which of these two possibilities actually occurs will be discussed in Part IV. In either case the eutectic clearly has formed a new phase, the $\beta$ phase.

Table 2 Thicknesses of the $\gamma^{\prime}$ lower band and the $\gamma_{\text {upper }}^{\prime}$ band according to the quench methods

\begin{tabular}{|c|c|c|c|c|c|c|}
\hline \multirow{2}{*}{ Quench media } & \multirow{2}{*}{ Exp. \# } & \multicolumn{2}{c|}{$\delta_{1}(\mu \mathrm{m})$} & \multicolumn{2}{c|}{$\delta_{2}(\mu \mathrm{m})$} \\
\cline { 3 - 7 } & & Center $\left(\delta_{1 \mathrm{c}}\right)$ & Edge $\left(\delta_{1 \mathrm{e}}\right)$ & Center $\left(\delta_{2 \mathrm{c}}\right)$ & Edge $\left(\delta_{2 \mathrm{e}}\right)$ \\
\hline \hline \multirow{2}{*}{ Ga-In } & 51 & 0 & 300 & 0 & 0 \\
\hline \multirow{4}{*}{ Water } & Up & 56,57 & 200 & 370 & 0 & 0 \\
\cline { 2 - 7 } & Ice-water & $48,50,49$ & 240 & 400 & $0-80$ & $0-40$ \\
\cline { 2 - 7 } & $\begin{array}{c}\text { Drop } \\
\text { (normal) }\end{array}$ & $\begin{array}{c}21,63,64,65, \\
94,114,27\end{array}$ & 160 & 330 & 350 & 200 \\
\hline \multicolumn{2}{|c|}{ Air } & 123 & 400 & 440 & 560 & 450 \\
\hline
\end{tabular}


a)

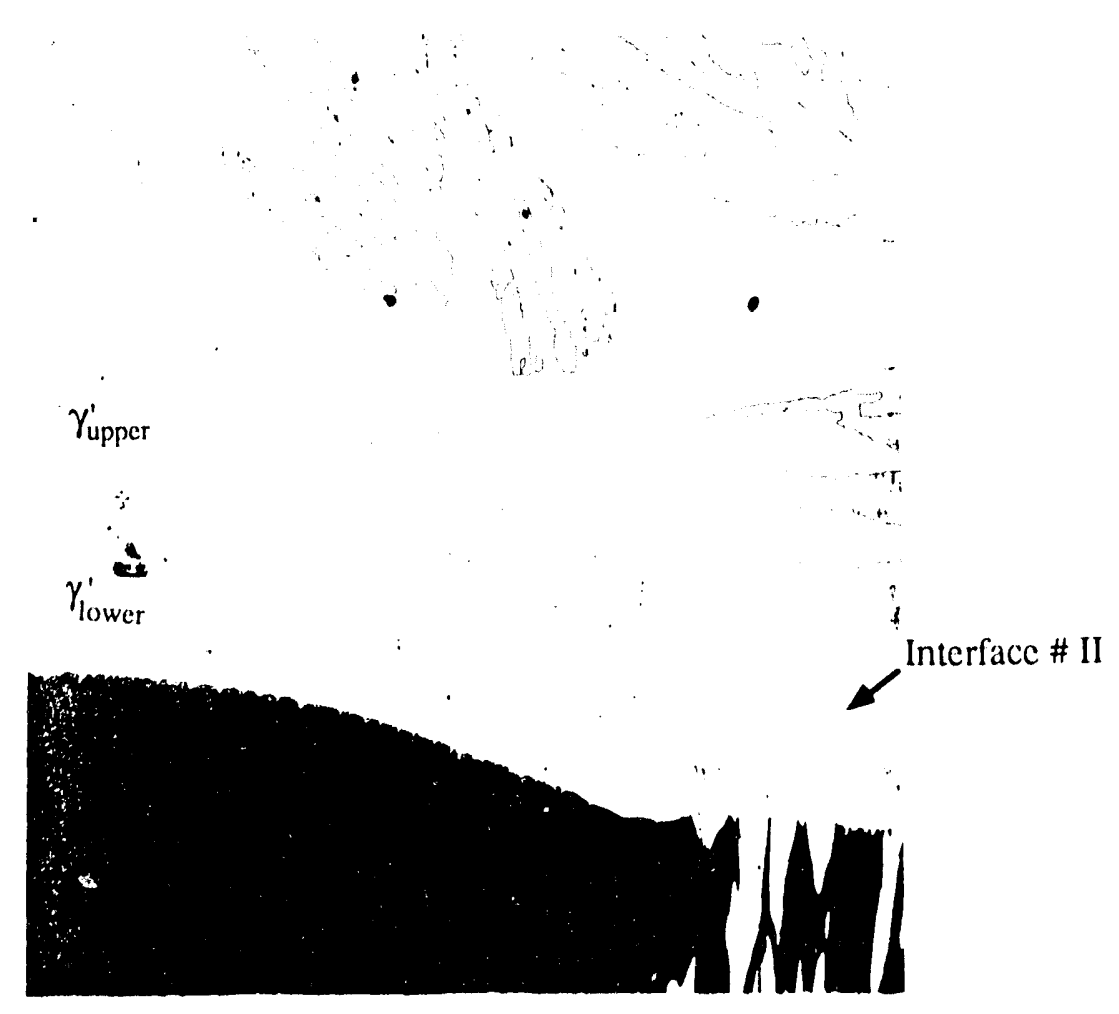

b)

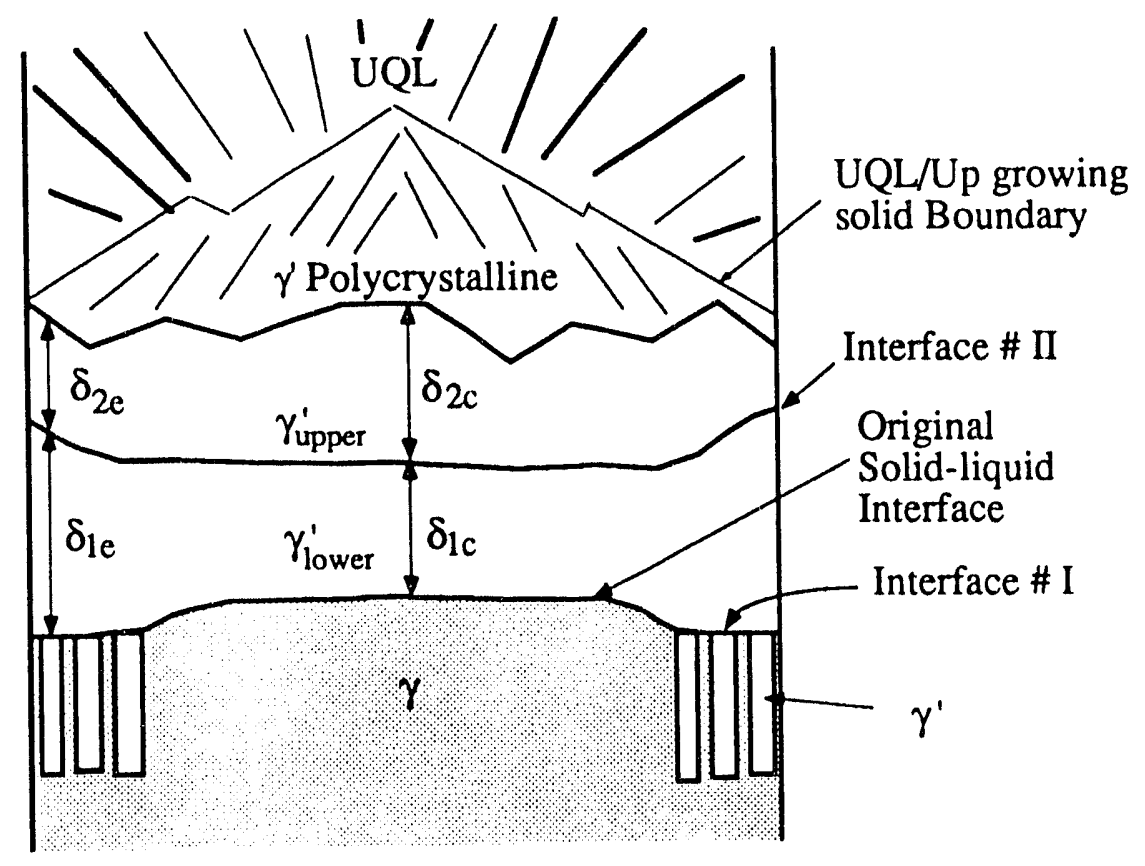

Figure 4. (a) High magnification in the edge region of Figure 3(a), 100x

(b) Schematic diagram of Figure 3(a) where the interfaces \# I and \# II, $\delta_{1}$, and $\delta_{2}$ are defined 
At the fastest quench rate of Ga-In quench (see Figure 3(d)), no $\gamma^{\prime}$ bands formed in the center region and the $\gamma^{\prime}$ lower bands formed only at the edge region, with no $\gamma^{\prime}$ upper band above it, i.e., $\delta_{2}$ was zero at the edge. This result provided strong evidence that the $\gamma^{\prime}$ band formed during the quench procedure and that the solid-liquid interface at the instant of quenching was located at the mystery interface \# I. Figure 3(d) shows that the $\gamma^{\prime}$ lower band forms more quickly near the container wall than at the center regions of the rod. The shape of the mystery interface \# II showed additional evidence that the $\gamma^{\prime}$ bands formed during the quenching procedure. The $\gamma^{\prime}$ lower band at the edge region always grew up more than that at the center region (compare $\delta_{1 \mathrm{e}}$ to $\delta_{\mathrm{lc}}$ in Table 2). This result makes sense because the quench rate of the edge part is faster than that of the center part. As the $\gamma^{\prime}$ lower band grows laterally across the solid-liquid front there is more time for longitudinal growth near the wall, and hence one expects the edge region of the band to grow further in the longitudinal direction before it stops growth. The shape of interface \#ll must not be the shape of the solid-liquid interface.

In the case where the solid-liquid interface was $100 \% \gamma$ phase it is not possible to form the lower $\gamma^{\prime} / \gamma^{\prime}$ mystery(\# I) interface. Initially it was thought that mystery interface \# II was also absent on such samples. However, with adequate etching the mystery interface \# II could be seen above most of the $\gamma / \gamma^{\prime}$ interface as is apparent in Figure 5(a) for an air quench and Figure 5(b) for a water quench.

It was very difficult to see the mystery interface \# I in experiments consisting of all $\gamma^{\prime}$ solid because this boundary was very dim in HF etching solution and almost nothing in Marbles etching solution, as shown in Figure 6. Note: The HF etch caused the microstructure above interface \# II to etch differently than that below, whereas this difference was much less with Marbles etch (compare Figure 6 (a) and (b)). In the $\gamma^{\prime}$ planar solid-liquid interface, the shape and existence of the mystery boundary II were the same with those of the $\gamma$ planar (with $\gamma+\gamma^{\prime}$ coupled phase) forming interface, but the mystery boundary \#I could not be found. It 
a)
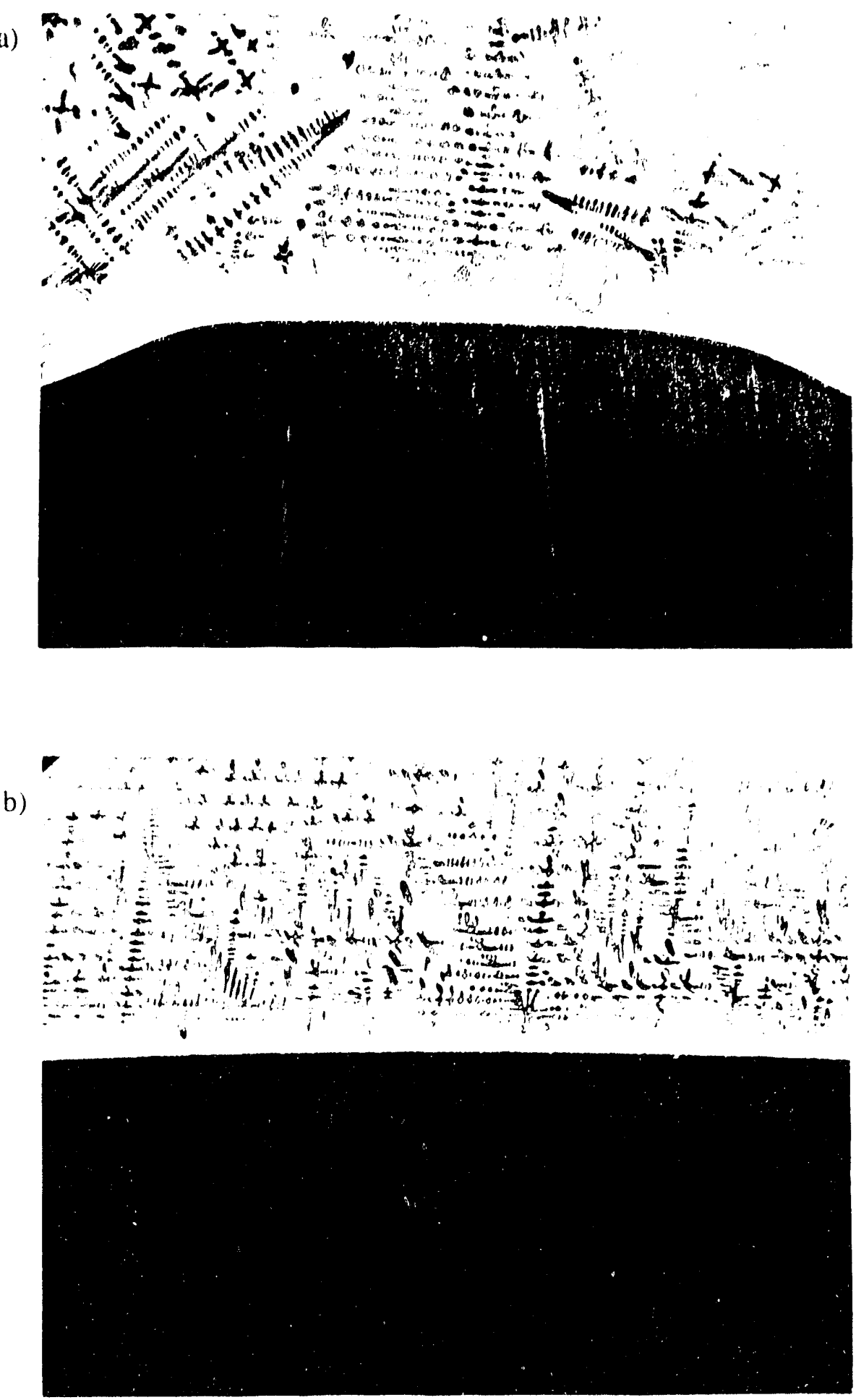

Figure 5. The $\gamma$ planar interfaces a) in cold finger quench (Exp.\#22, Al 23-().8 $\mu \mathrm{m} / \mathrm{s}$,

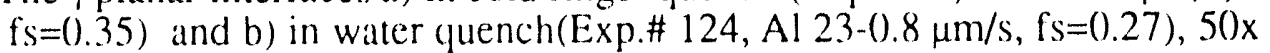


a)

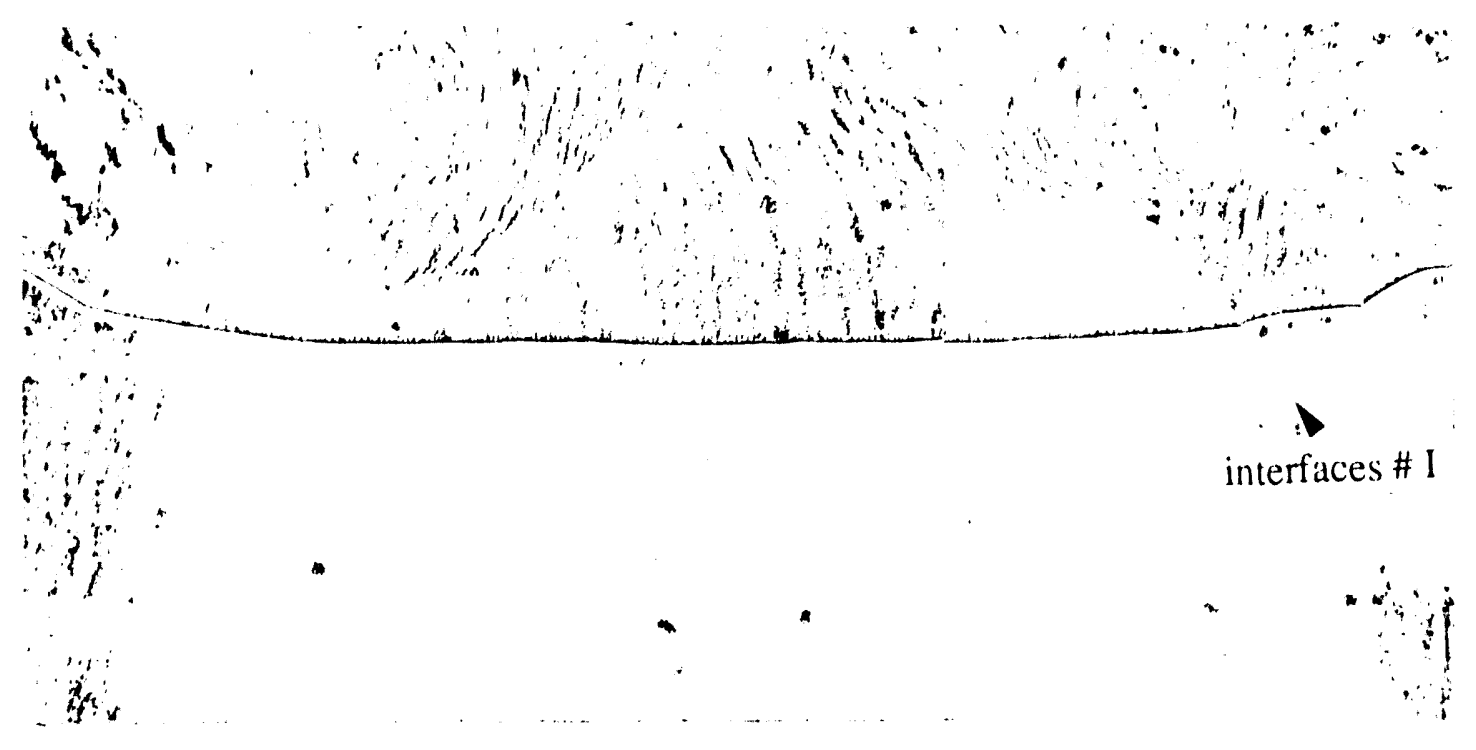

b)

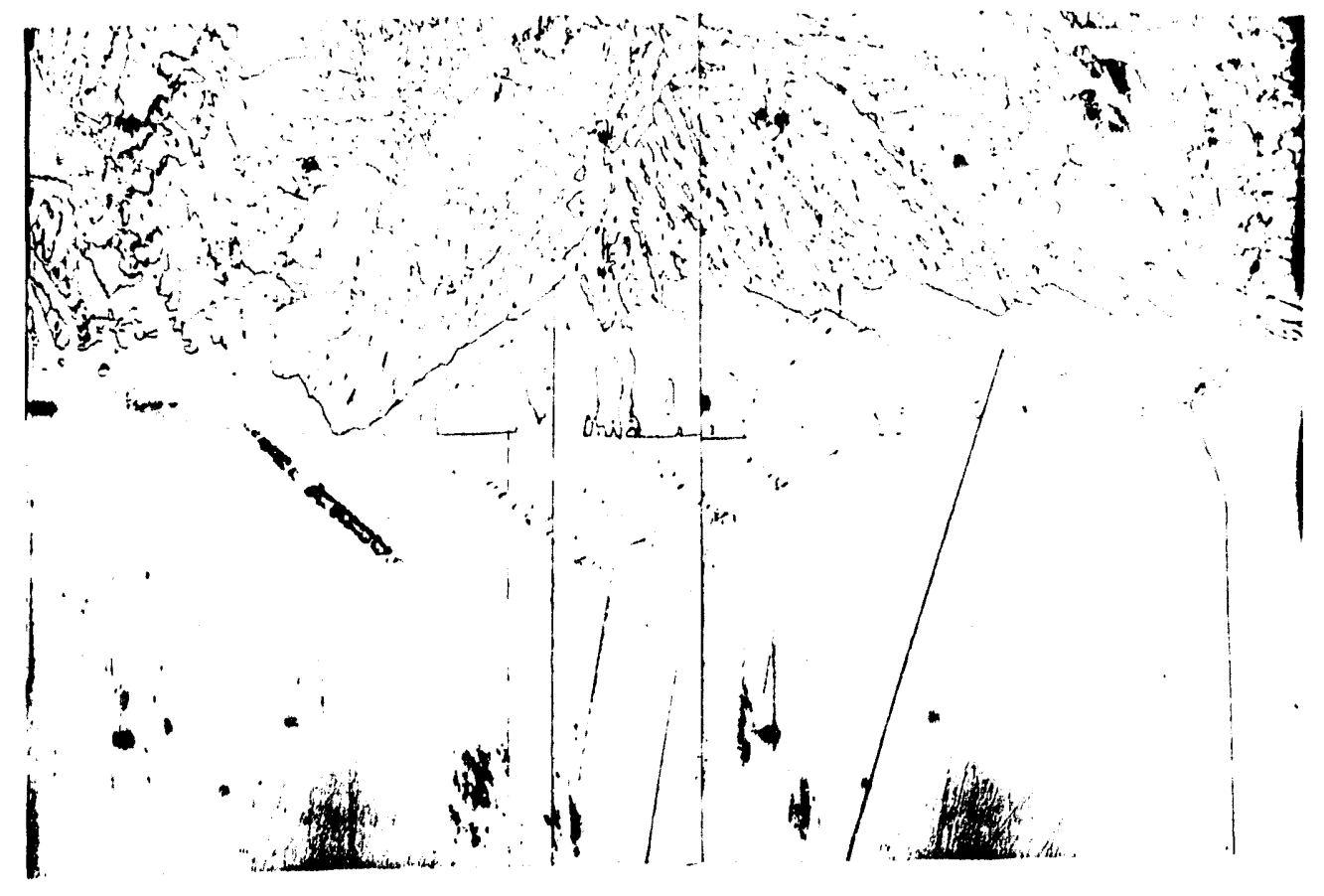

Figure 6. The $\gamma^{\prime}$ lower band in front of the $\gamma^{\prime}$ planar interface of Al $24-0.8 \mu \mathrm{m} / \mathrm{s}$-ice water quench (Exp.\# 49). This sample was etched in a) HF etching solution for 1 hour and b) Marbles solution for 20 second, 50x 
seems that the mystery boundary \# I forms clearly only when the $\gamma^{\prime}$ phase exists with the $\gamma$ phase at the solid-liquid interface for any quench condition.

These quench rate control experiments prove that $\gamma^{\prime}$ bands form on quenching in front of (a) a $\gamma$ planar interface with and without $\gamma+\gamma^{\prime}$ phases at its edges, (b) a $\gamma+\gamma^{\prime}$ planar interface, or (c) a $\gamma^{\prime}$ planar interface. However, the nature of the mystery boundaries was unknown. It was suspected to result from some orientation or structure change at the interfaces \# I and \#II, and further investigations were followed in TEM and SEM.

Investigations of Orientations, Crystal Structures, and Transmitted Image

The crystallographic orientations were investigated on the region $\mathrm{A}$ ( $\gamma^{\prime}$ upper band), the region B ( $\gamma^{\prime}$ lower band), and the $\gamma$ and $\gamma^{\prime}$ phases of the region $C$ of Figure $7(a)$ in SACP (Selected Area Channeling Pattern). All the positions showed the same orientation, as shown in Figure 7(b). This orientation, perpendicular to the longitudinal section, was 10 degrees in $x$ and 10 degrees in $y$ off from $<112>$. If the orientations of the region $A, B$, and $C$ were different, the mystery boundaries were simply grain boundaries. However, these two boundaries were not grain boundaries and so they were named mystery boundaries. Further investigations were required in the TEM.

The orientations were also examined in TEM. The observed positions are shown in Figure 8. The transmitted dark and bright field images in region A, $\gamma_{\text {upper }}$ band, and B, $\gamma^{\prime}$ lower band, were the same, as shown in Figure 9. The region $C$ of the $\gamma$ planar interface displayed a net-work shape consisting of minor $\gamma$ regions in a $\gamma^{\prime}$ matrix, as shown in Figure 10. This net-work shape seems reasonable in region $\mathrm{C}$ because the $\gamma^{\prime}$ is expected to precipitate in the $\gamma$ phase. By comparing the $\gamma^{\prime}$ phase in the band of region B to the $\gamma^{\prime}$ precipitated $\gamma$ phase in region $C$ using the SEM, it was proved that the major phase of region $C$ was precipitated $\gamma^{\prime}$ and the net-work shaped minor phase was the $\gamma$ phase. The SAD (Selected 
a)

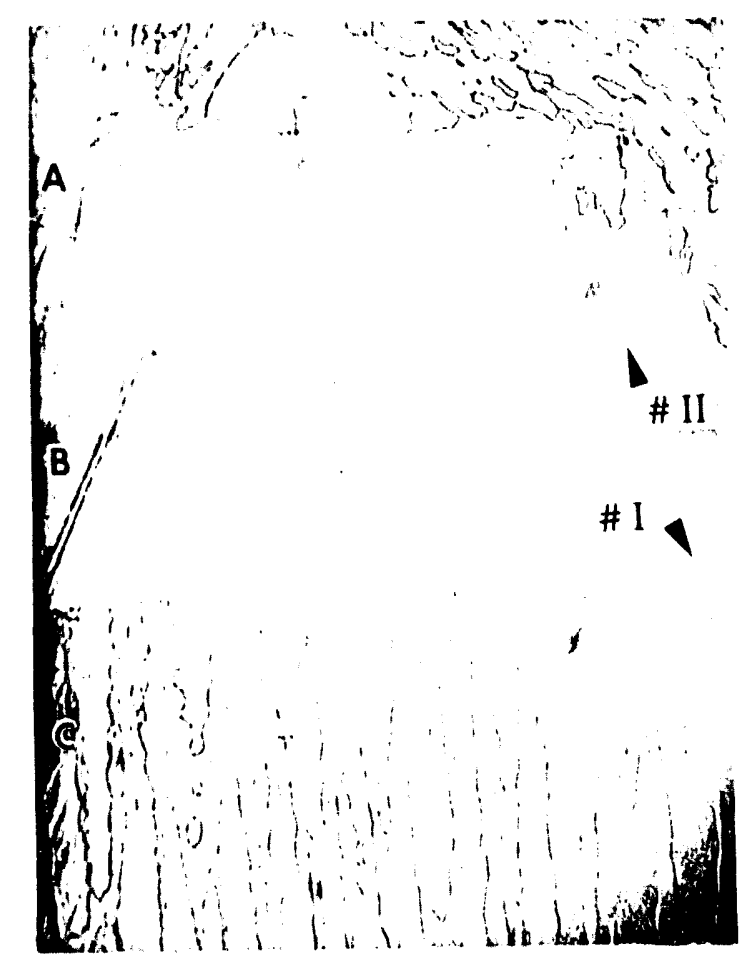

b)

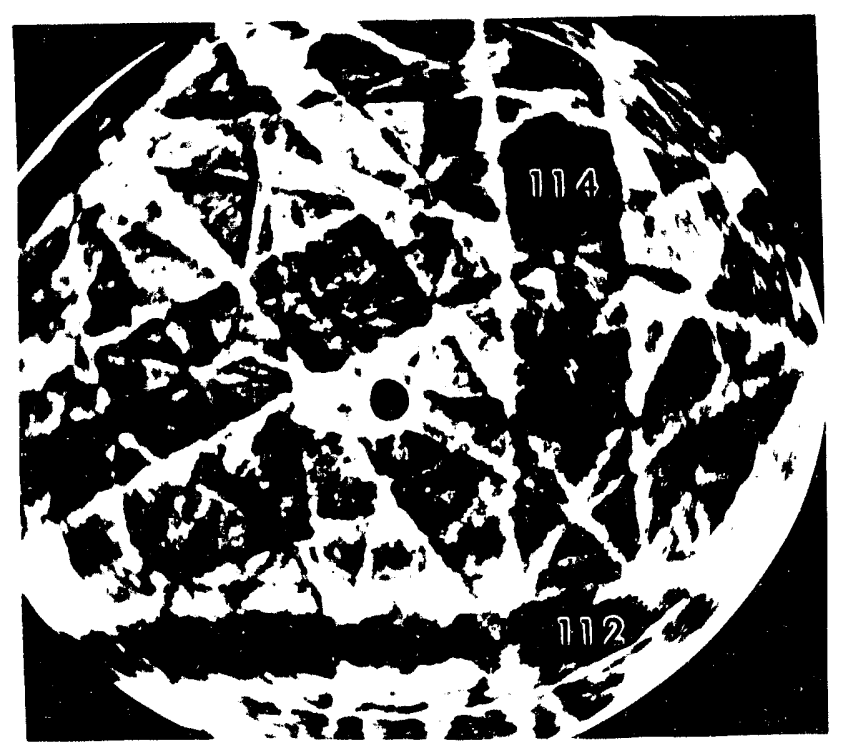

Figure 7 a) The positions of SACP in the solid-liquid interface region in Exp. \# 64

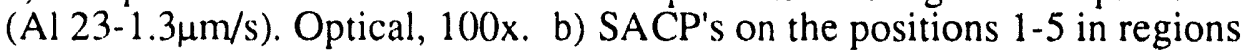
$A\left(\gamma^{\prime}\right.$ upper band), $B\left(\gamma^{\prime}\right.$ lower band), and $C$ (solidified $\left.\gamma+\gamma^{\prime}\right)$. The orientations of all the positions are the same 


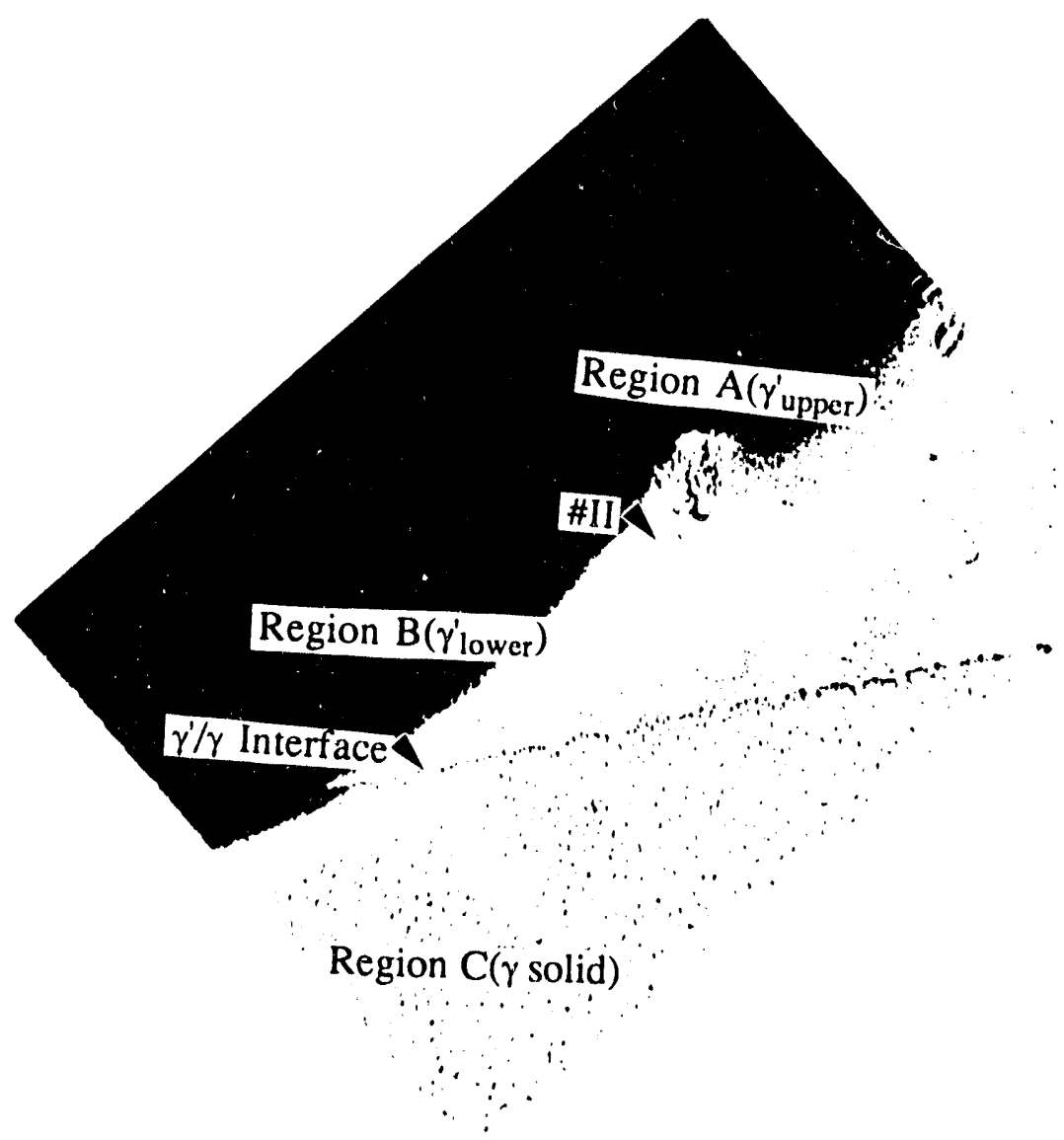

Figure 8 The $\gamma / \gamma^{\prime}$ interface and the $\gamma^{\prime} / \gamma^{\prime}$ mystery interface \#II in the TEM foil of Exp. \# 21 (Al 23-0.8 $\mu \mathrm{m} / \mathrm{s}), 200 \mathrm{x}$ 
a)

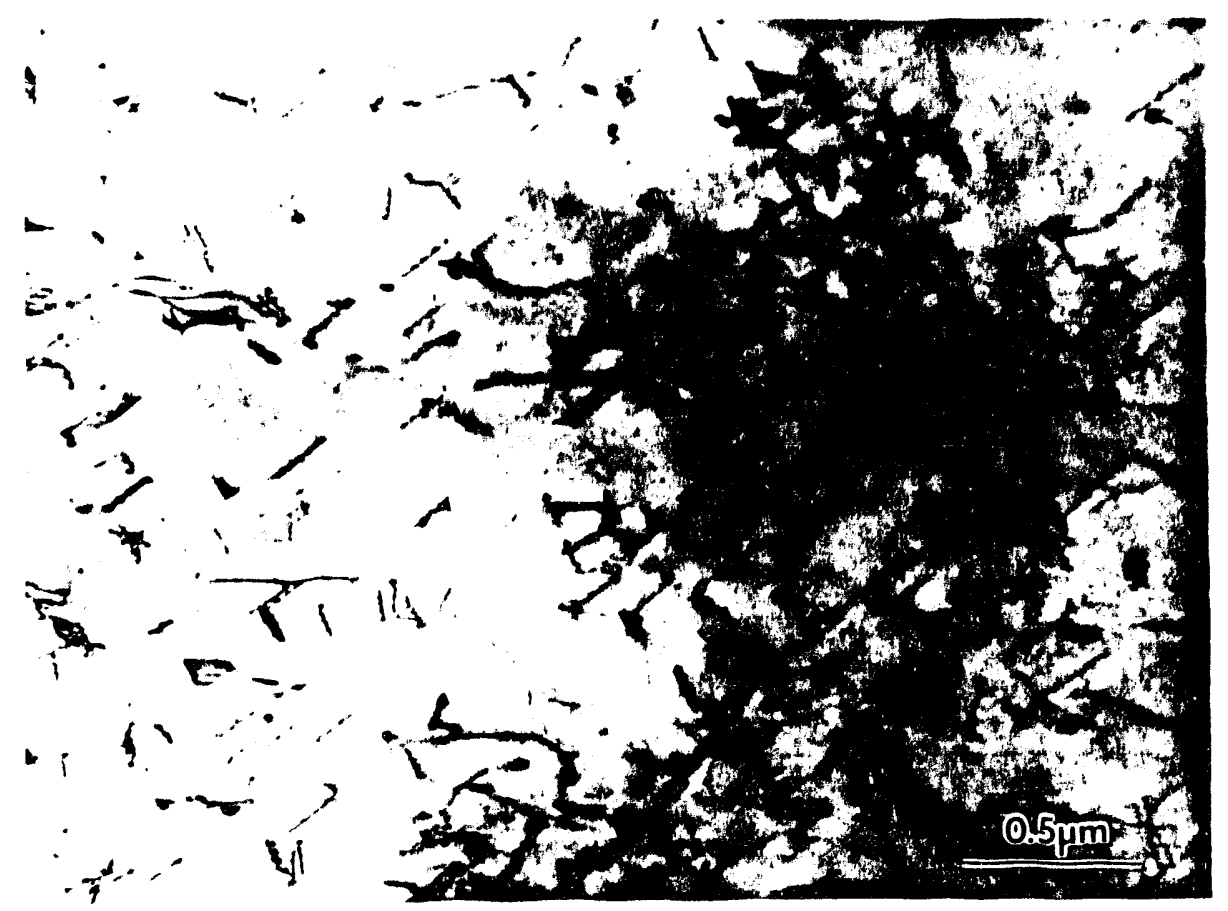

b)

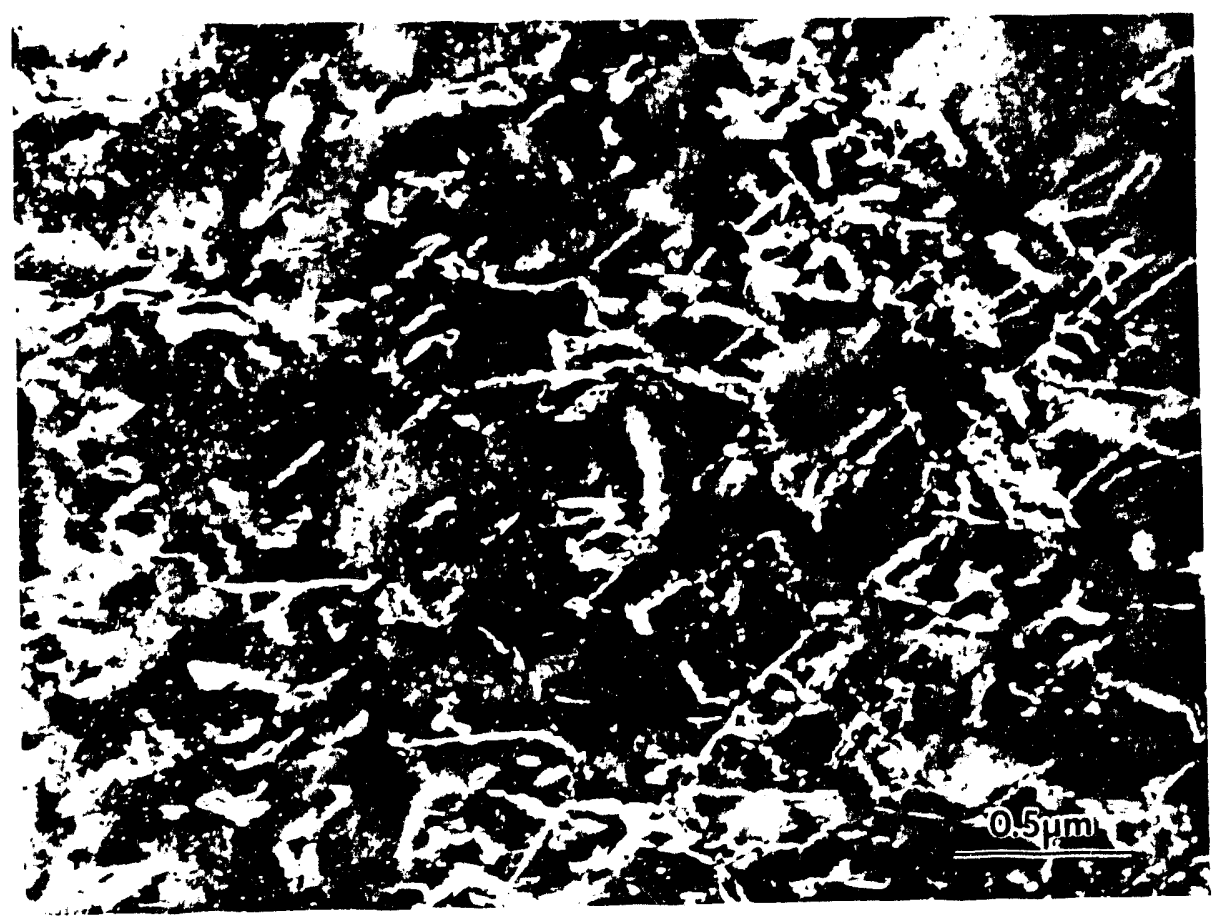

Figure 9 Transmitted image pattens a) at bright field and b) at dark field in region A, $\gamma_{\text {upper }}$ band, on weak beam condition 
c)

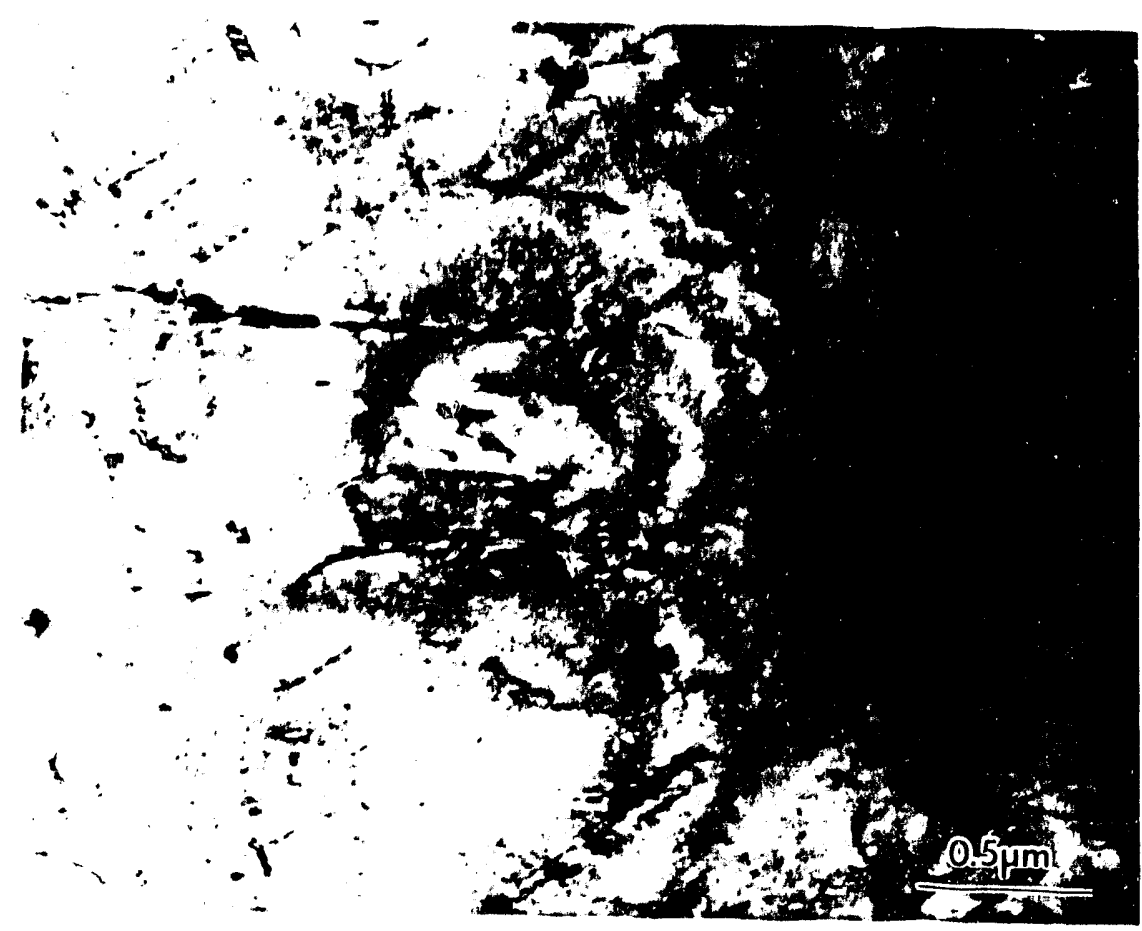

d)
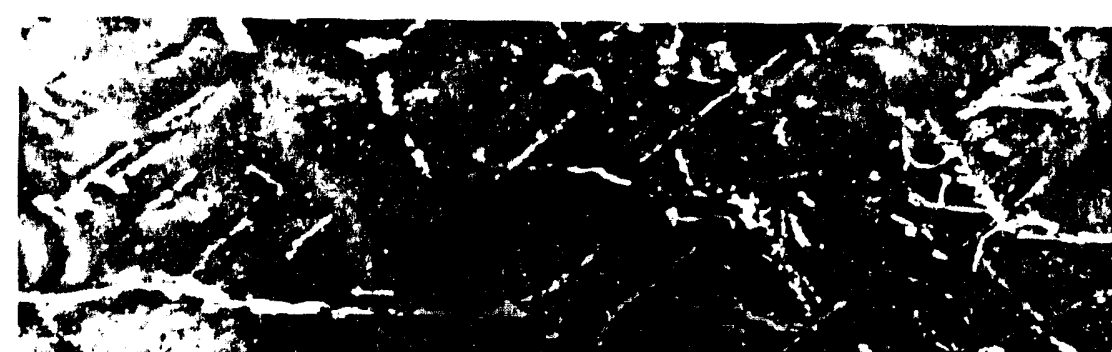

$18+2$

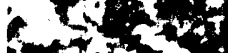
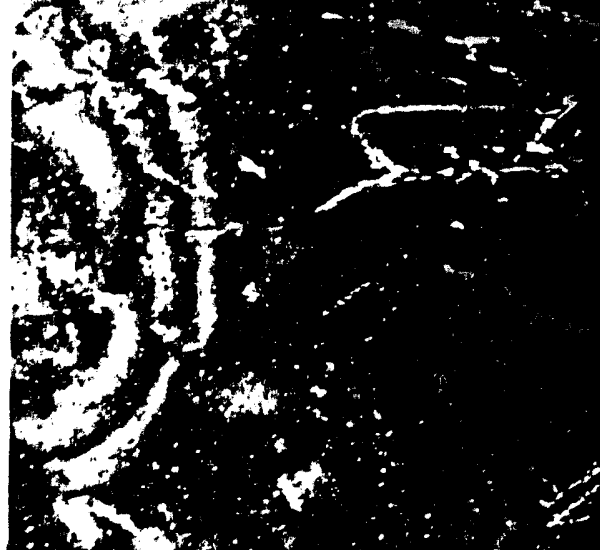

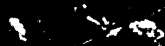
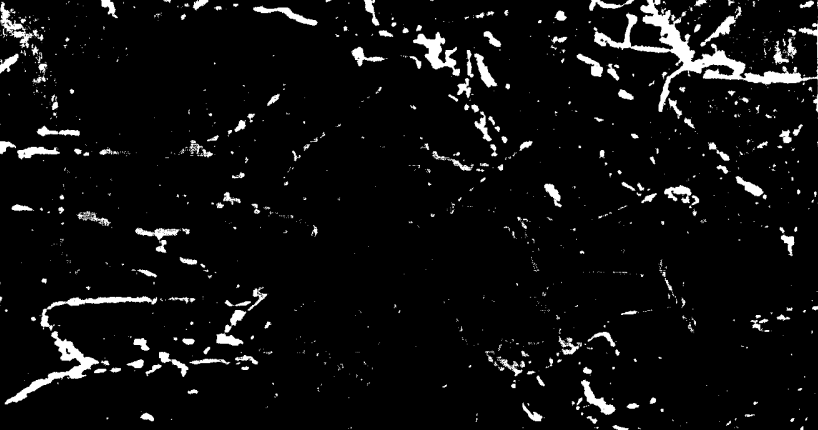


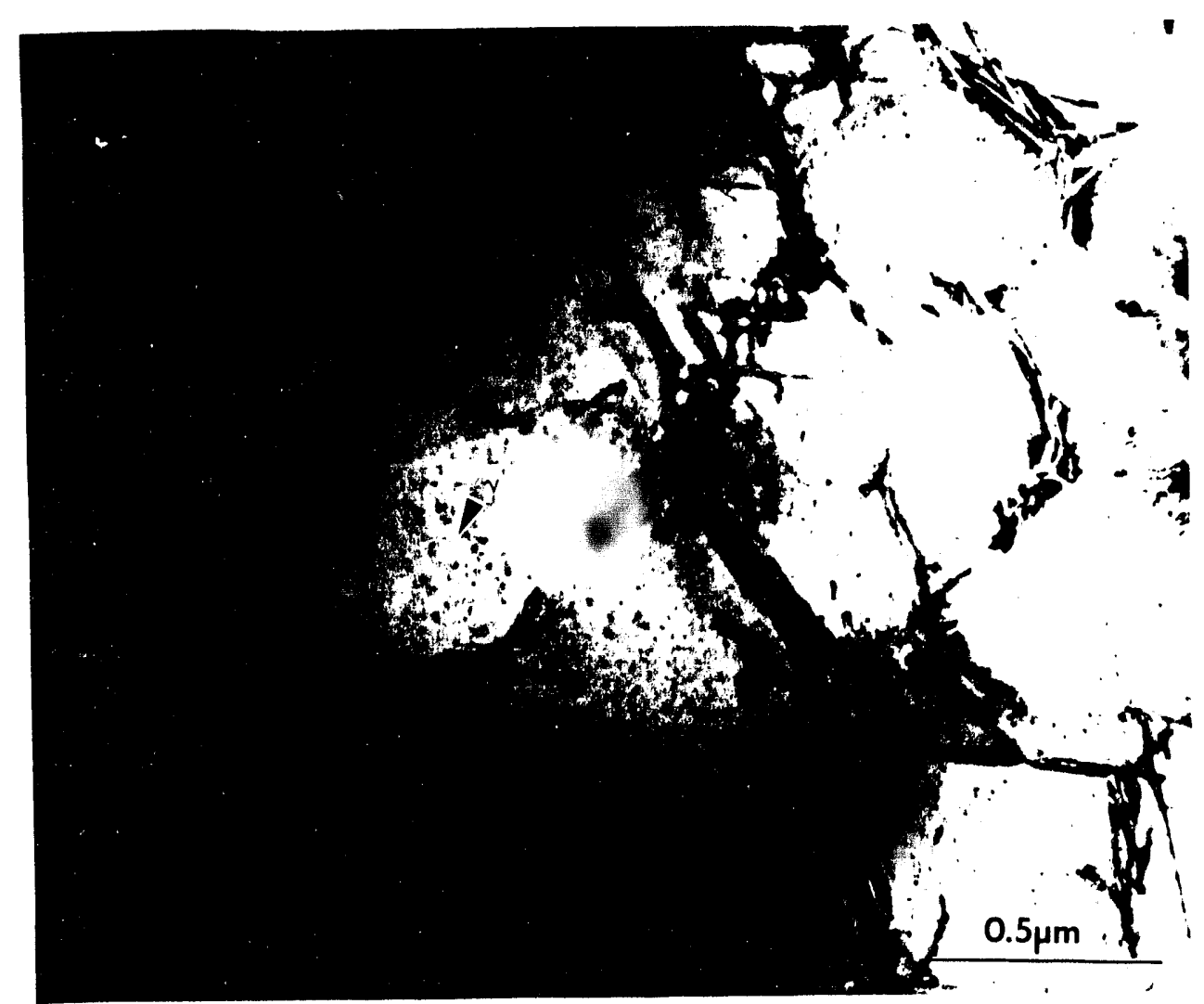

Figure 10. Transmitted image pattern at bright field on region $C$ (solidified $\gamma$ phase) 
a)

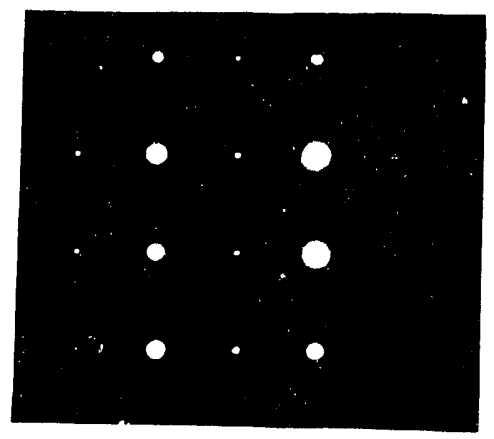

b)

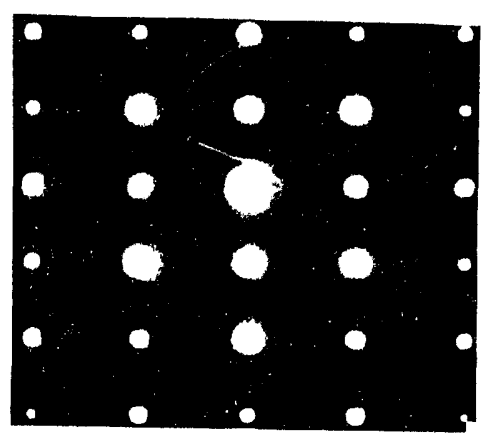

c)

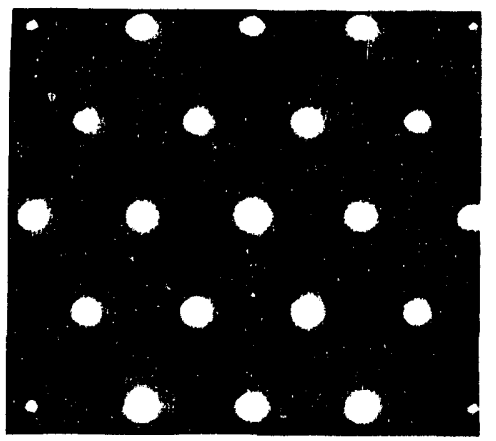

Figure 11. SAD patterns at different zone axis ; a) $\langle 112\rangle$, b) $\langle 110\rangle$, and c) $\langle 111\rangle$. These patterns are the same on all three $A, B$, and $C$ regions 
Area Diffraction) patterns on the region $\mathrm{A}, \mathrm{B}$, and $\mathrm{C}$ were all of the same orientation and all were the ordered FCC structure of $\gamma^{\prime}$. The SAD patterns were obtained on the different zone axis: $\langle 112\rangle,\langle 110\rangle$, and $\langle 111\rangle$, as shown in Figure 11. A convergent beam diffraction technique, which is more sensitive to structure change, was also used on region A and B at the $<111>$ zone axis. The convergent beam diffraction patterns on region $\mathrm{A}$ and $\mathrm{B}$ were identical as shown in Figure 12. The region around the mystery boundary \# II was carefully examined in high magnification transmitted image patterns, but any trace of a boundary revealed by fringe patterns was not found. The $<112>$ zone axis could be obtained by tilting 9 degrees in $\mathrm{x}$ and 12 degrees in $y$. This orientation agreed with the orientation obtained by the channeling pattern in the SEM.

Another TEM sample (Exp. \# 94) as shown in Figure 13, which includes interfaces I and II, was prepared using a $100 \% \quad \gamma+\gamma^{\prime}$ coupled planar interface, after some studying to establish conditions for the $\gamma+\gamma^{\prime}$ coupled growth. Any fringe pattern evidence of the interfaces \# I and \#II was not found in the high magnification images and the orientation on each region $(A, B, C)$ was also the same. The sample was examined in bright field with a small aperture near several major zone axes under conditions expected to show fringe patterns at boundaries, but no fringe patterns appeared. Figure 14 shows the $\gamma$ phase which was found in the $\gamma+\gamma^{\prime}$ two phase $\mathrm{C}$ region. This $\gamma$ phase region was the same net-work structure as found for the $\gamma$ phase region in the $\gamma$ planar interface of Figure 10.

It was very strange result that the interfaces \# I and \# II were observed in optical microscope but not in TEM. The TEM analysis proves that the interfaces \#I and II are not boundaries induced by structural or orientational changes. Also, the possibility of an atomistic plane of local disorder on the interfaces could be rejected because fringe patterns were not observed. 
a)

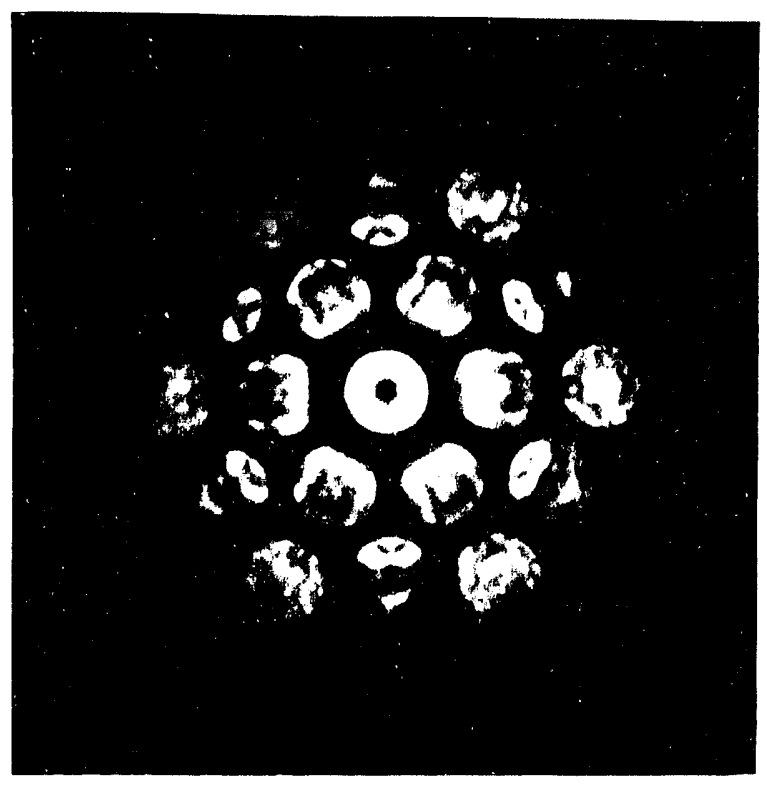

b)

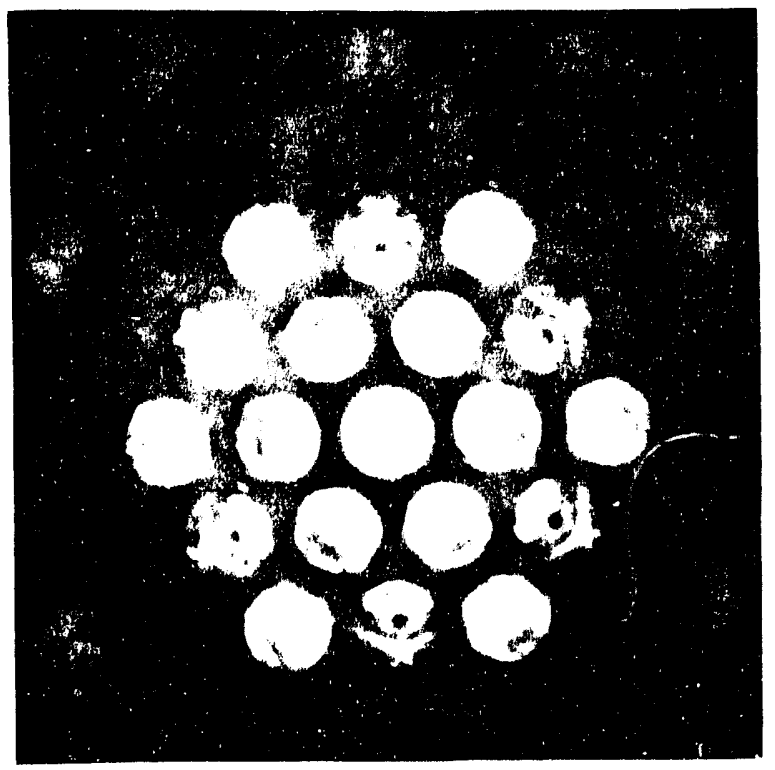

Figure 12 Convergent beam diffraction patterns on a) region $\mathrm{A}$ and $\mathrm{b}$ ) region $\mathrm{B}$. These patterns are identical 


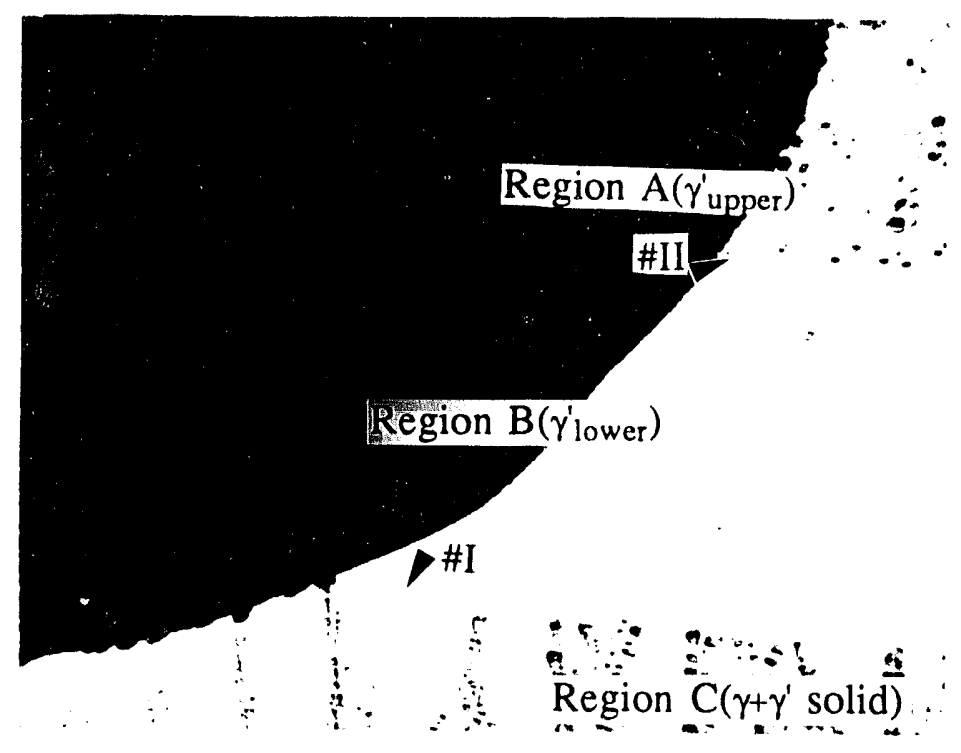

Figure 13 The observed positions in TEM sample. The $\gamma+\gamma^{\prime}$ planar, $\gamma^{\prime}$ bands, interfaces \#

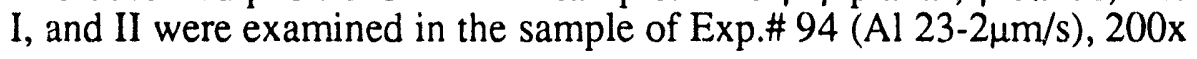


a)

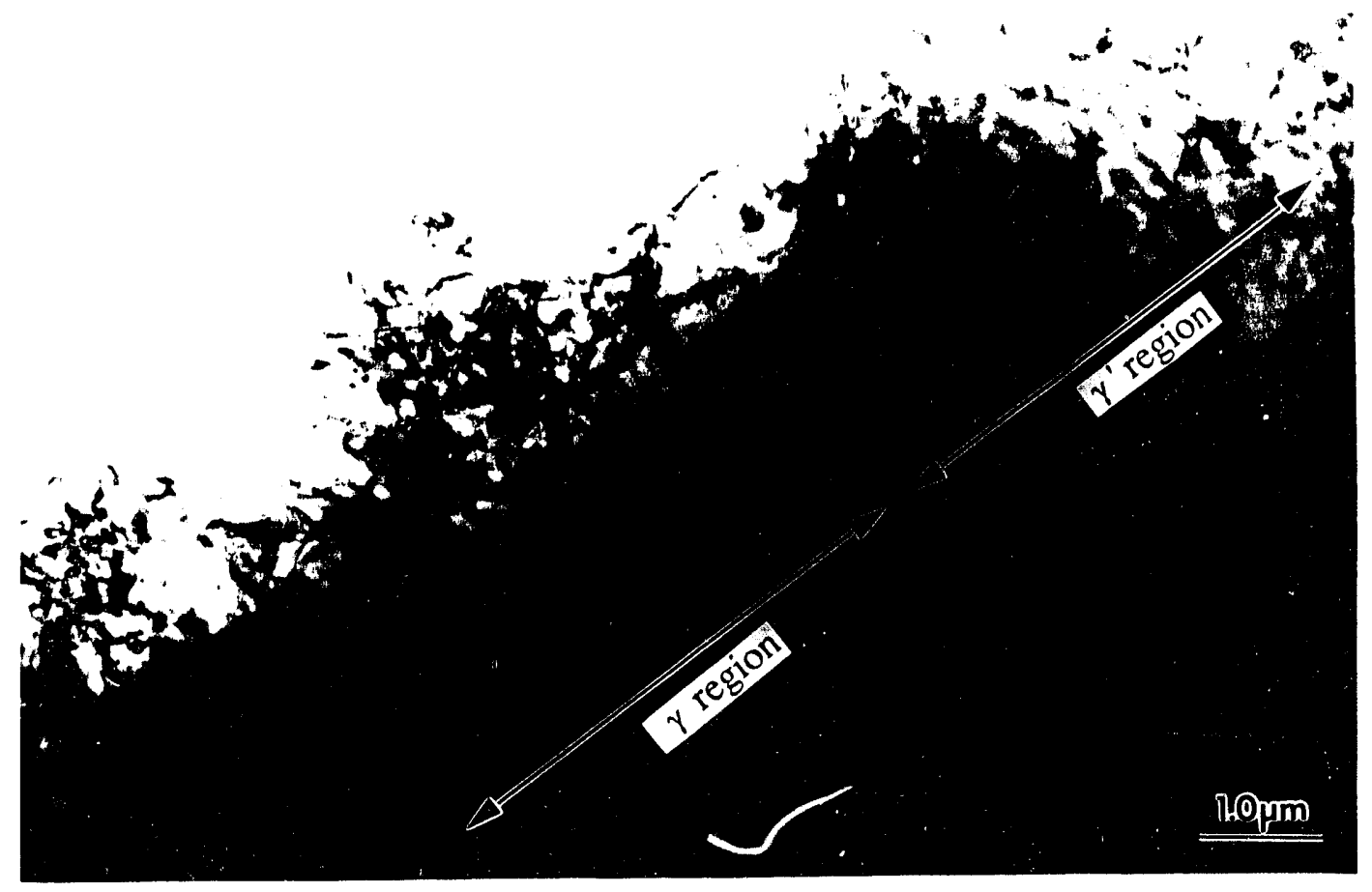

b)

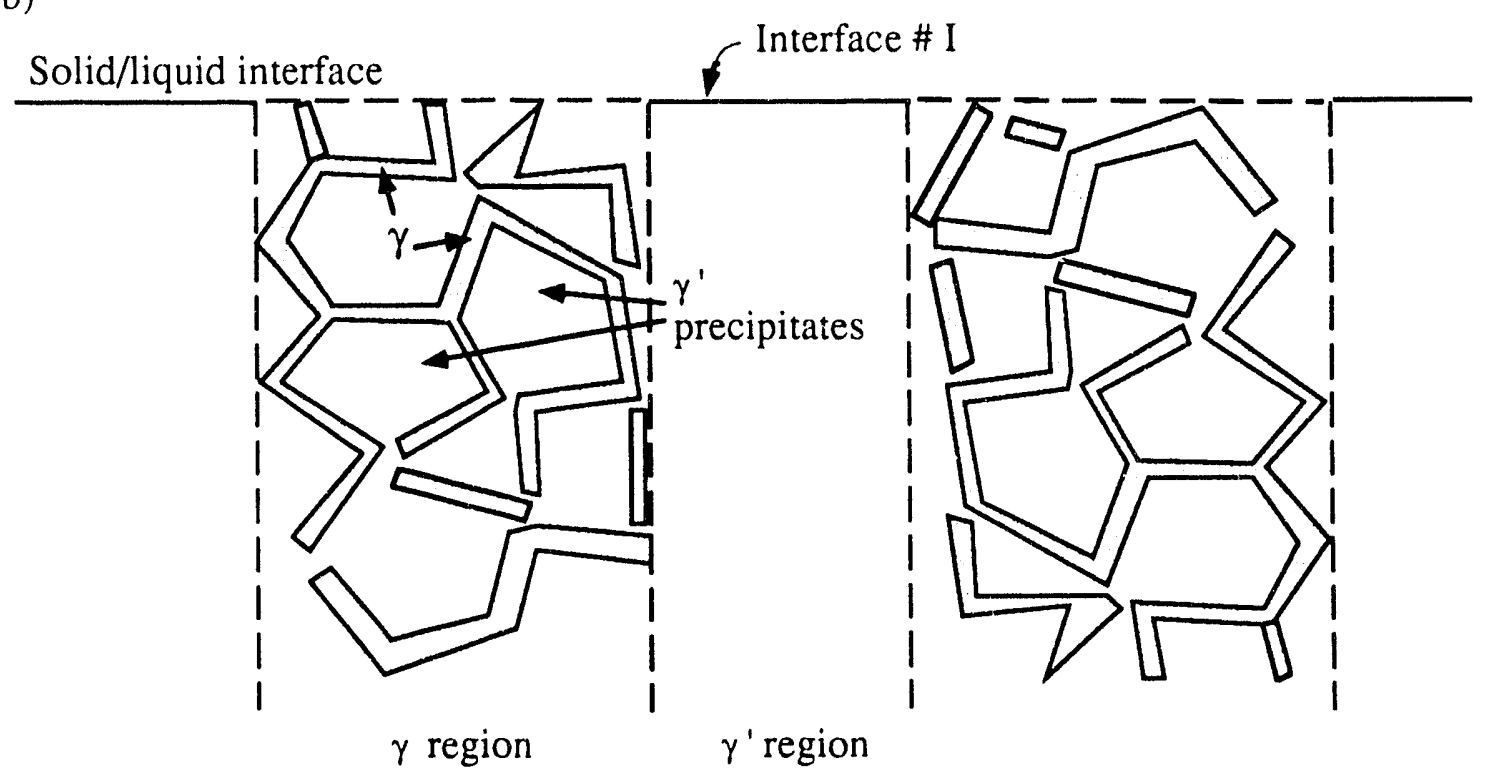

Figure 14. a) Transmitted image patterns in the $\gamma+\gamma^{\prime}$ coupled structure of the sample of Exp.\# 94 , where the $\gamma$ phase region includes $\gamma$ precipitates and its structure is the same with Figure 10 , and b) schematic diagram of the $\gamma+\gamma^{\prime}$ coupled struture 
Composition Analysis on the Mystery Boundaries in the SEM and TEM

The composition change was analyzed at the solid-liquid interface region by EDS in the SEM. It was analyzed from the $\gamma$ planar interface to the quenched liquid through the $\gamma^{\prime}$ lower band and mystery interface \# II on the center region of Figure 3(a). No significant composition change was found across the mystery boundary \# II, while there was about a 3 at.\% Al difference at the $\gamma^{\prime}$ lowerband / $\gamma$ planar interface, as shown in Figure 15 . The composition change across the mystery interface \# I (on the edge region of Figure 3(a)) was examined but a composition change could not detected, where the EDS was used at $15 \mathrm{kV}$. The diameter of sample volume producing $\mathrm{x}$-ray was about $2 \mu \mathrm{m}$ at this voltage [1]. If it is a very local composition change (less than $1 \mu \mathrm{m}$ ) on the mystery interface, it is too small to pick up on regular EDS.

The composition variations on the interfaces \#I and \#II were analyzed in steps of 0.2 $\mu \mathrm{m}$ by EDS in the TEM, where the beam size was $52 \mathrm{~nm}$. Figure 16(a) shows that the relative Al composition was high on the interface \# II. However, any significant composition variation on the interface \# I was not detected. It was difficult to analyze on the interface \# I in this foil because the edge of the hole was nearly parallel to the interface \#I, as shown in Figure 13. In order to cross the interface \# I at right angles, the sample thickness varied significantly along the direction of beam motion. The result shown in Figure 16(b) revealed no local composition change at the interface, perhaps because the relative $\mathrm{Al}$ intensity was decreased according to the thickness.

A low voltage technique was used in EDS of the SEM and a maximum volume size producing $x$-ray could be expected by the following equation [1],

$$
R \rho=0.064\left(E_{0}^{1.68}-E_{c}^{1.68}\right)
$$

where $R$ is a $x$-ray production range in $\mu \mathrm{m}, \rho$ is the density of the element in $g / \mathrm{cm}^{3}, E_{c}$ is the critical ionization energy for characteristic $x$-rays in $\mathrm{kV}$, and $\mathrm{E}_{0}$ is the accelerating voltage in $\mathrm{kV}$. The expected maximum range was about $0.3 \mu \mathrm{m}$ at $5 \mathrm{kV}$, while it was $2.2 \mu \mathrm{m}$ at $15 \mathrm{kV}$. 


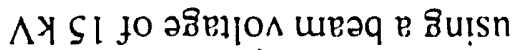

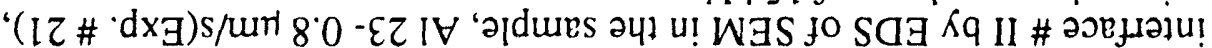

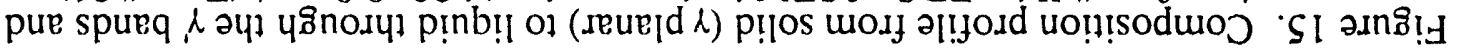

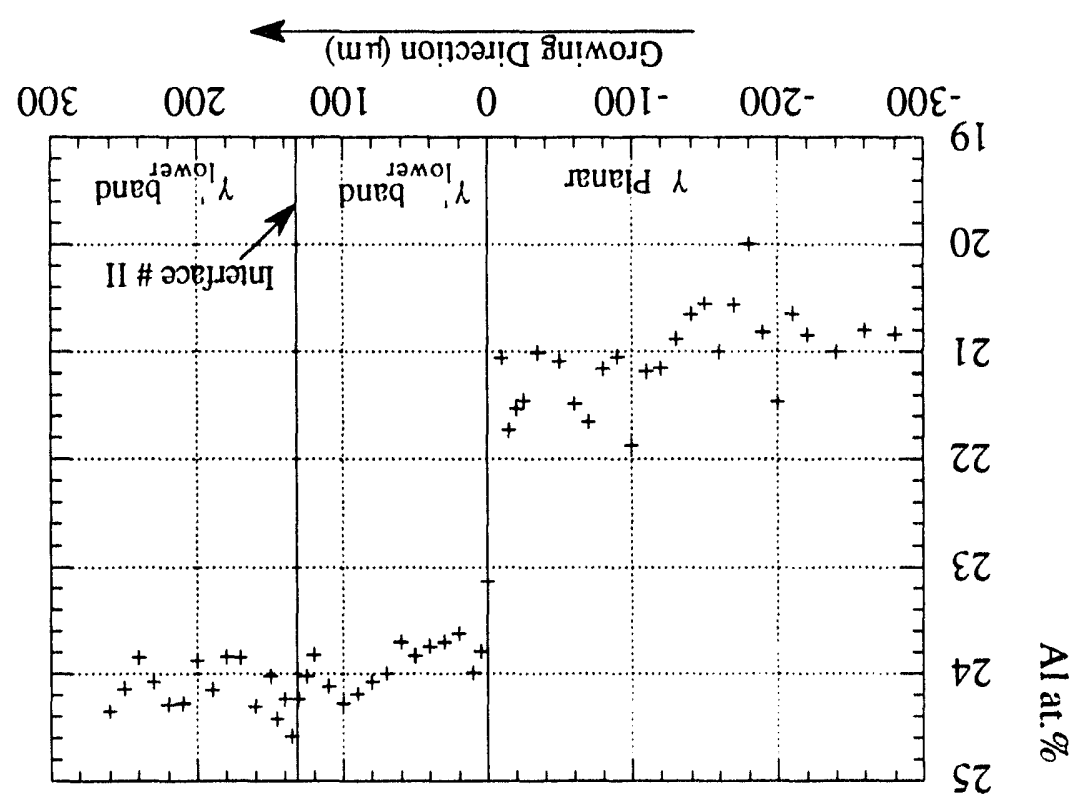


a)

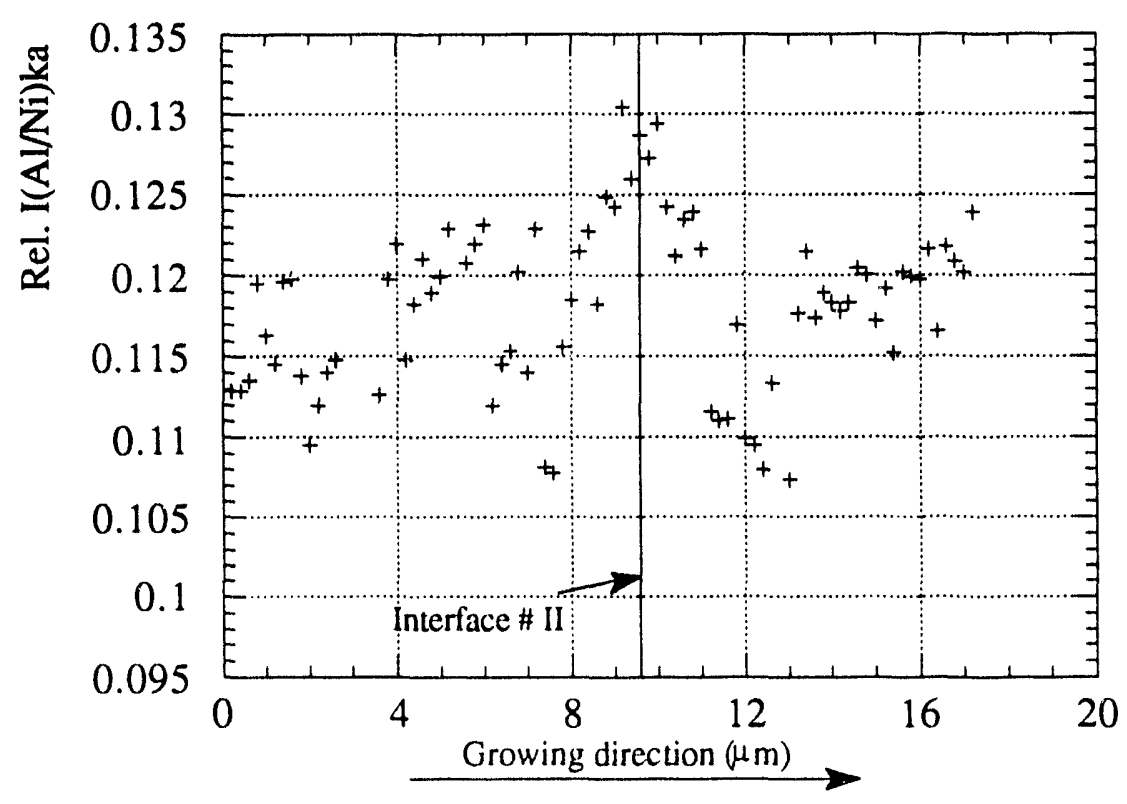

b)

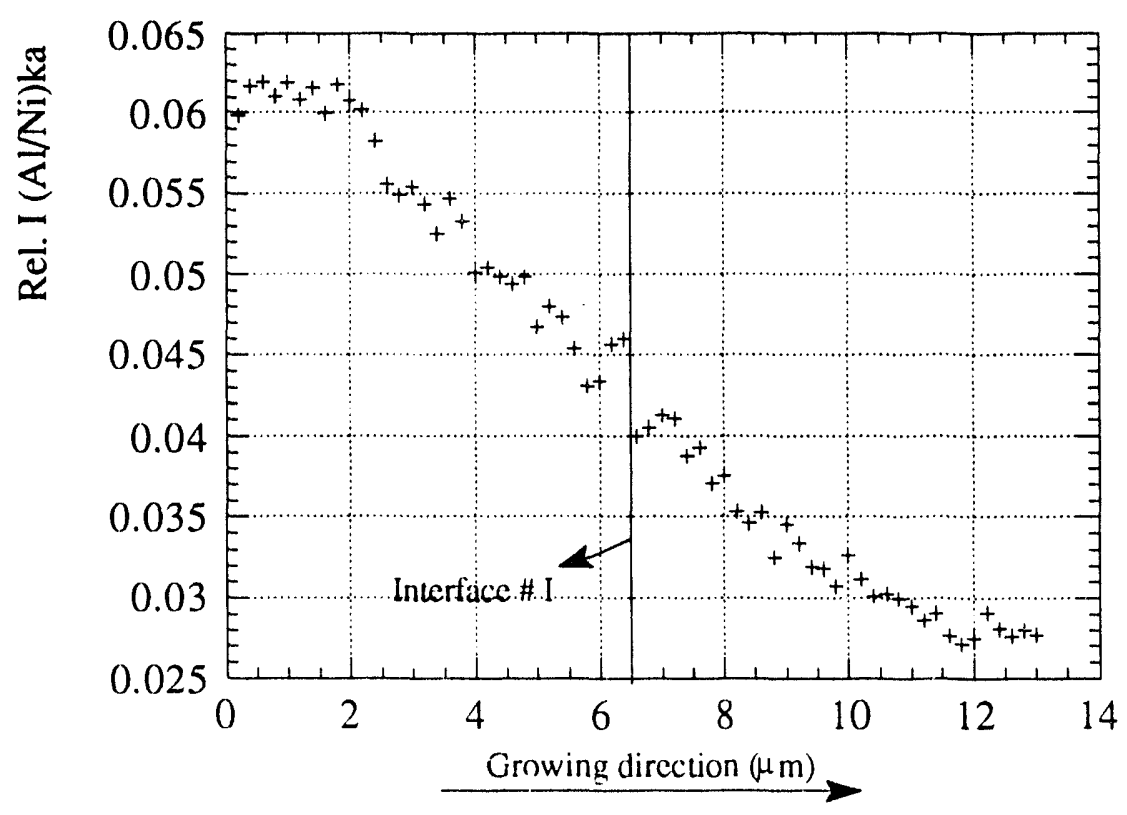

Figure 16. Composition variation a) at the interface \# II and b) \# I in the foil of Figure 13(Exp.\# 94) by EDS in the TEM 
a)

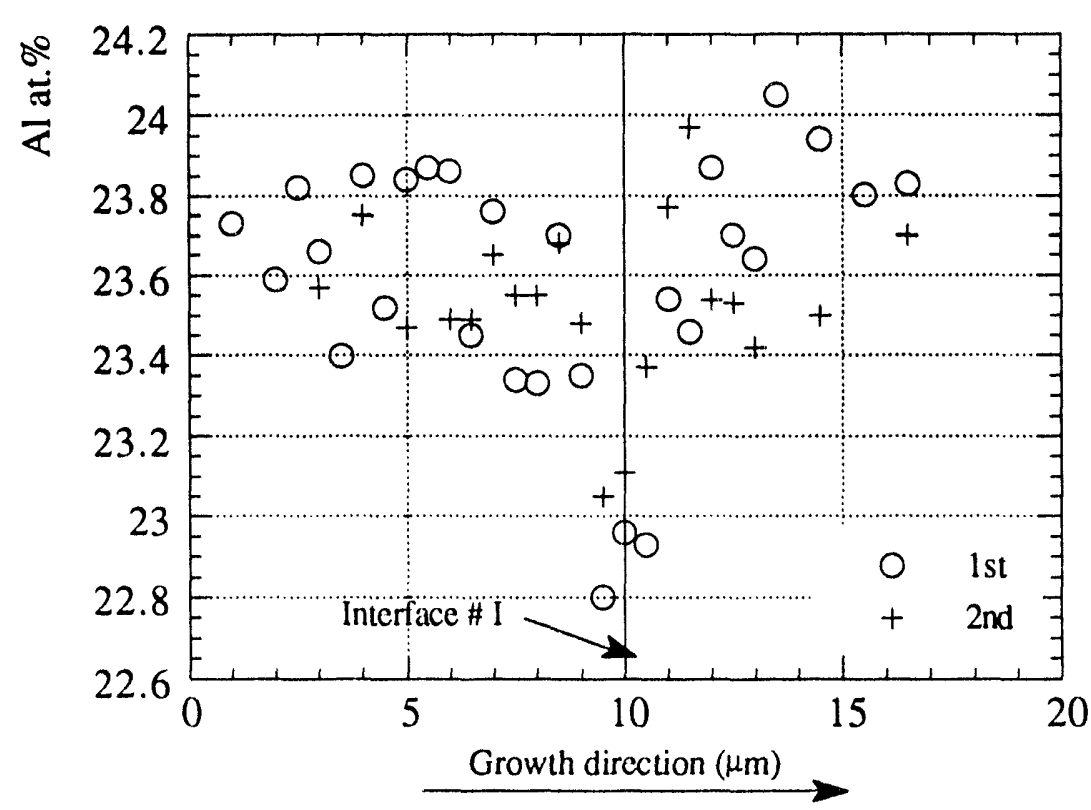

b)

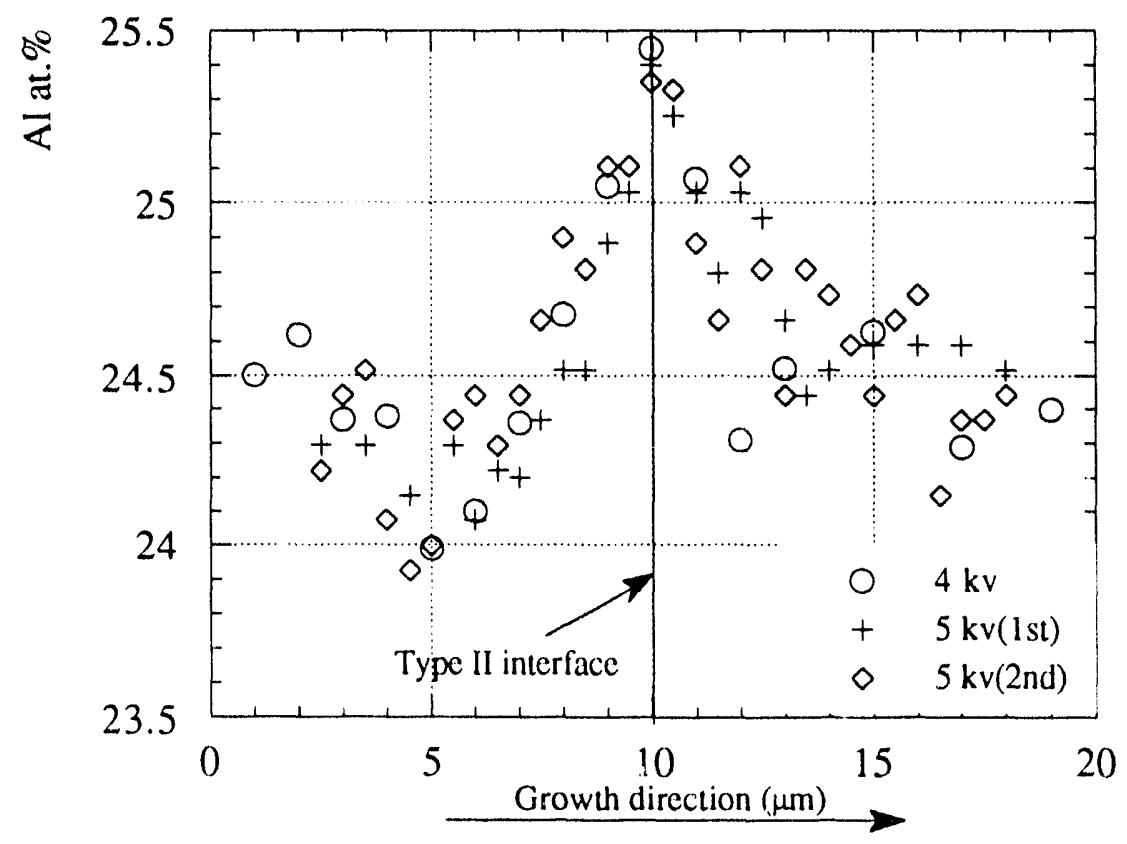

Figure 17. Composition variation along different locations at a) the interface \# I and b) \# II by EDS in the SEM at 4 and $5 \mathrm{kV}$ 
Figure 17 shows the EDS results on the interfaces \# I and II at 4 and $5 \mathrm{kV}$. The Al

composition decreased on the interface \# I and increased on the interface \# II. It was deduced from the data that the range of segregat on at interface \# II was less than $3 \mu \mathrm{m}$ and it was less than $1 \mu \mathrm{m}$ at interface \# I. If these mystery interfaces results from a local composition variation, they should be removed by heat-treatment. The mystery interfaces were heat-treated to see how these interfaces would change.

\section{Heat-treatment}

A series of heat-treatments was carried out in the samples of Exp.\# $64(1.3 \mu \mathrm{m} / \mathrm{s})$, Exp.\# $65(1.3 \mu \mathrm{m} / \mathrm{s})$, and $\operatorname{Exp} \# 94(2 \mu \mathrm{m} / \mathrm{s})$ on Al 23 alloys. These results are summarized in Table 3. The mystery boundaries disappeared at $1000-1200^{\circ} \mathrm{C}$. Figure 18 shows the $\gamma^{\prime}$ band regions before and after heat-treatment. The orientations of the interface region were also examined before and after heat-treatment by SACP to observe any orientation change at the original boundary location. The orientations of the region $A, B, C$, and D (in Figure 18) remain the same before and after heat-treatment, as shown in Figure 19. Region A is the "up growing solid", region B is the $\gamma_{\text {upper }}$ band, region C is the $\gamma^{\prime}$ lower band, and region D is the solidified $\gamma$ region. Recrystallization occurred in the "up gorwing solid" region (region $\mathrm{A}$ ) as well as UQL (upper quenched liquid) at $1200^{\circ} \mathrm{C}$, but the mystery boundaries dissapeared at $1000^{\circ} \mathrm{C}$ without changing orientation at the locations of the mystery interfaces. The interface region near the edge of this sample is compared before and after heat-treatment in Figure 20, where SACP showed that the positions of $\mathrm{B}, \mathrm{C}, \mathrm{D}$, and $\mathrm{D}^{\prime}$ maintained the same orientation and recrystallization occurred in the "up growing solid" and $U Q L$ regions $A, A^{\prime}$, and $A^{\prime \prime}$ after heattreatment. The interface \# II could be also removed at $1090^{\circ} \mathrm{C}$ in the sample of Exp. 65 .

Figure 21 shows clearly that both interfaces \# I and \# II have disappeared after heat treatment at $1000{ }^{\circ} \mathrm{C}$ in the $\gamma+\gamma^{\prime}$ planar interface of Exp. 94. These experiments show that the mystery interfaces have disappeared upon heating even though the crystallographic orientation at the 
original boundary locations has not changed. This result supports the local composition change as the cause of interfaces \# I and \# II.

Table 3 Heat-treatment of the mystery interfaces

\begin{tabular}{|c|c|c|c|c|c|}
\hline Exp.\# & Interface & Temp $\left({ }^{\circ} \mathrm{C}\right)$ & Time(h) & Interface \# I & Interface \# II \\
\hline \multirow[t]{7}{*}{64} & $\begin{array}{l}\gamma \text { planar with } \\
\gamma+\gamma^{\prime} \text { coupled }\end{array}$ & 400 & 1 & No change & No change \\
\hline & & 490 & 2 & " & " \\
\hline & & 590 & 4 & $"$ & $"$ \\
\hline & & 700 & 4 & " & $"$ \\
\hline & & 900 & 4 & Dim & Dim \\
\hline & & 1000 & 1 & Disappeared & Disappeared \\
\hline & & 1200 & 3 & " & $"$ \\
\hline \multirow[t]{4}{*}{65} & $\gamma^{\prime}$ planar & 1000 & 0.5 & -... & No change \\
\hline & & 1050 & 0.8 & $-\cdots$ & Dim \\
\hline & & 1070 & 0.8 & $\cdots$ & Dim \\
\hline & & 1090 & 1 & $\ldots$ & Disappeared \\
\hline 94 & $\gamma+\gamma^{\prime}$ planar & 1000 & 2 & Disappeared & Disappeared \\
\hline
\end{tabular}


(e wodf

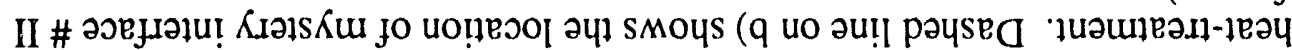

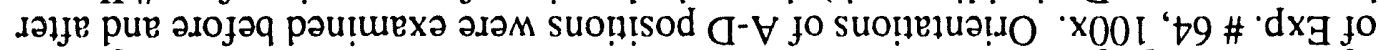

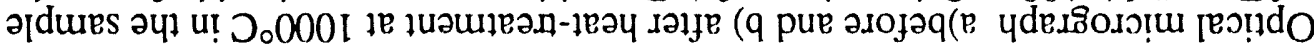

8โ ว.กถิโป

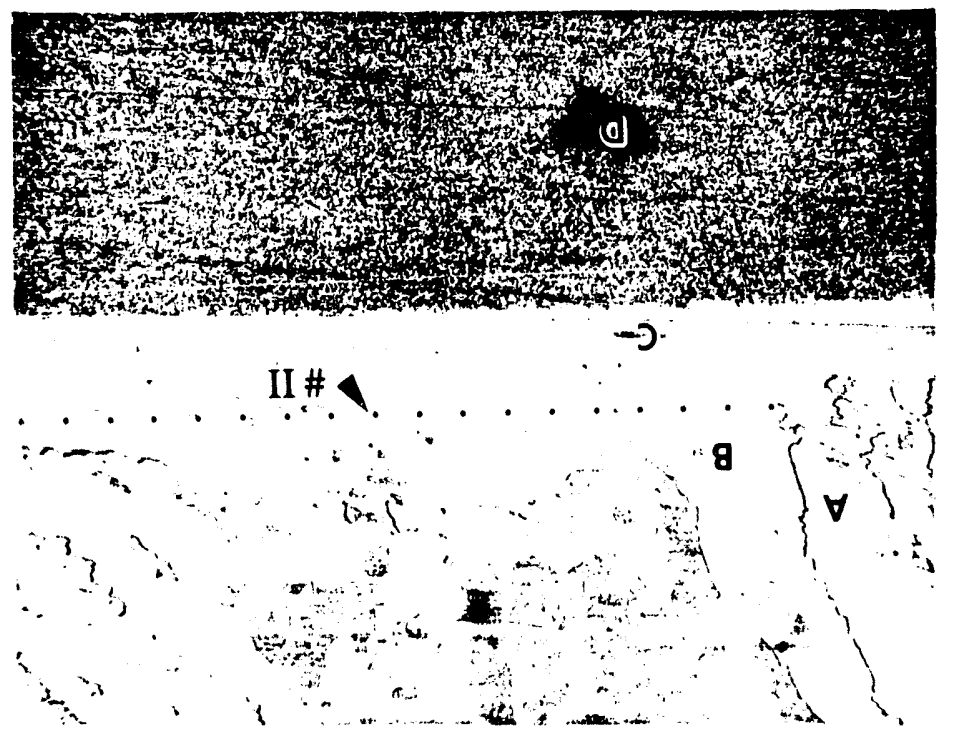

$(9$

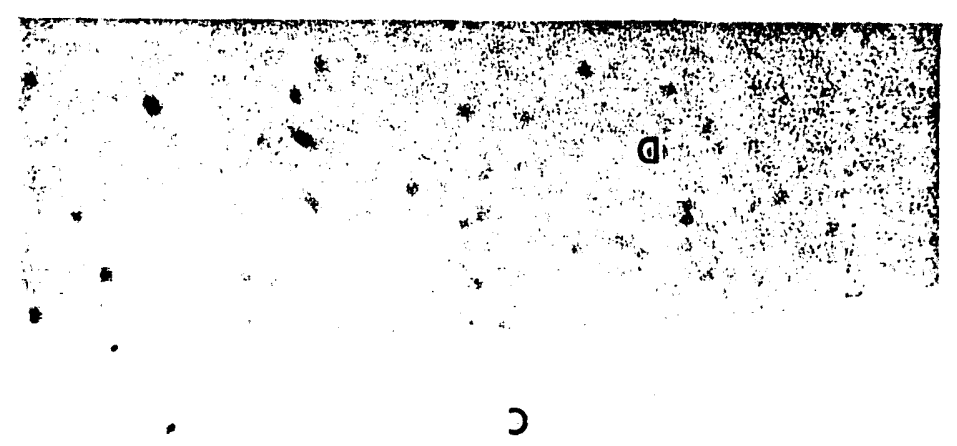

II\# 4

8

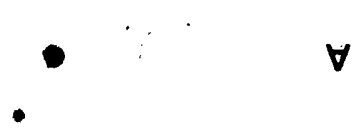


a)

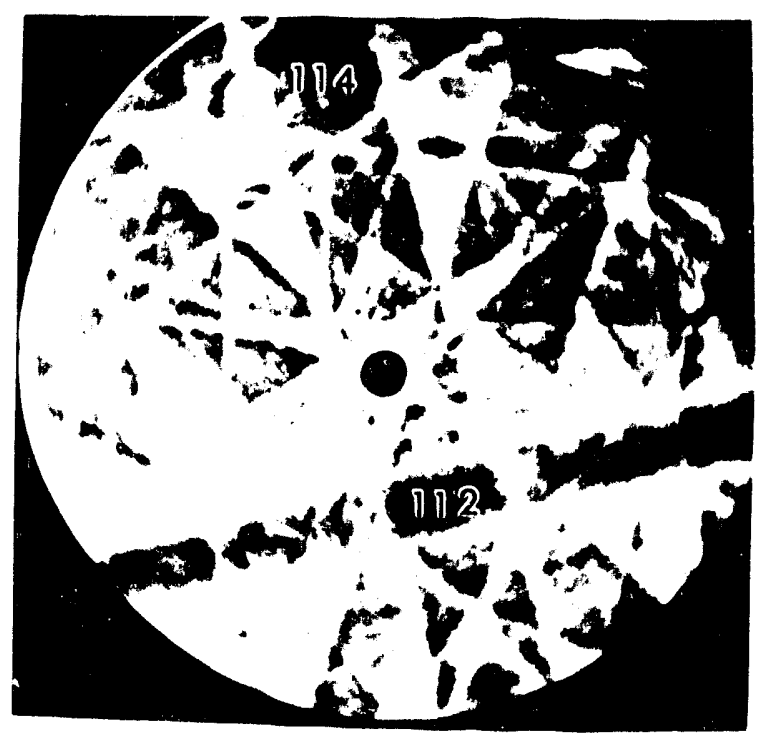

b)

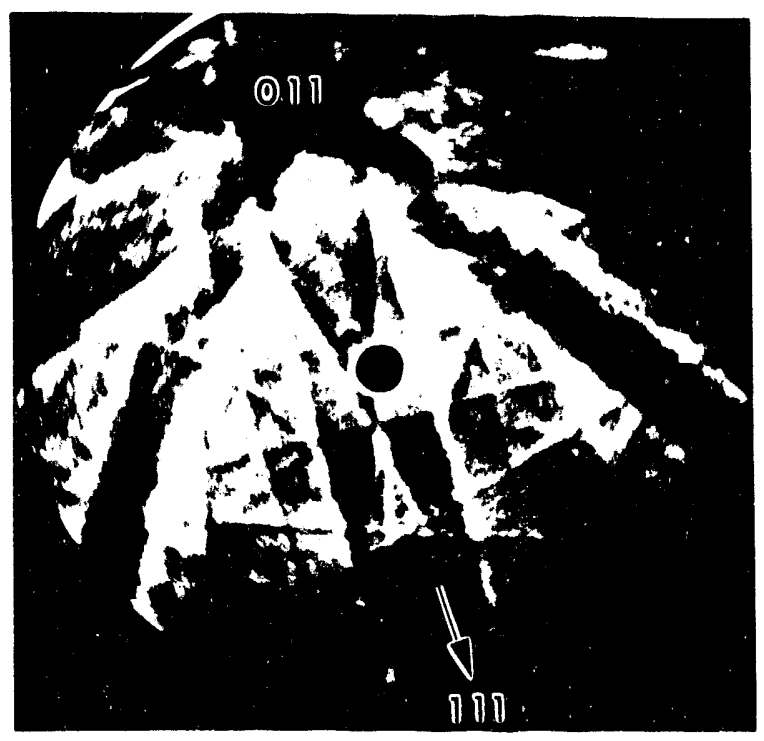

Figure 19 SACP's on each position of Figure 18 at a) A, b) B,C, and D 


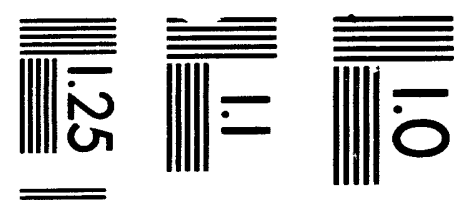

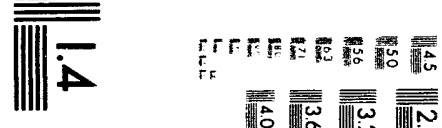

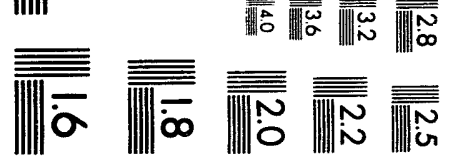



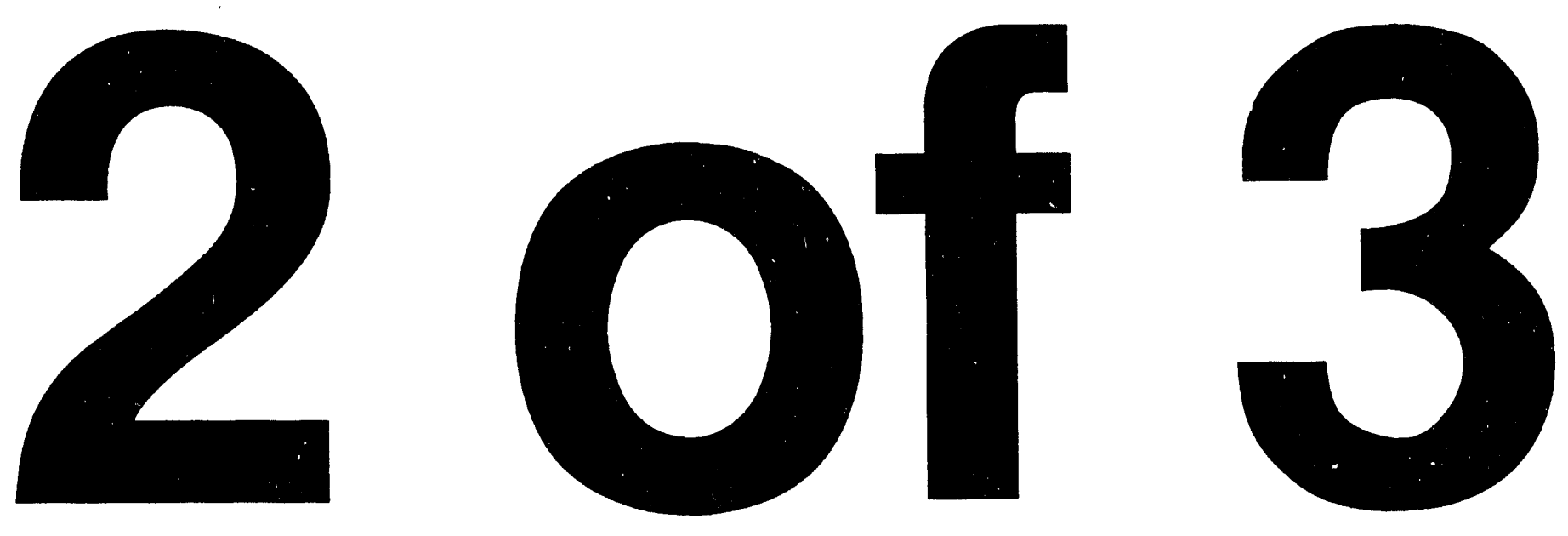
a)

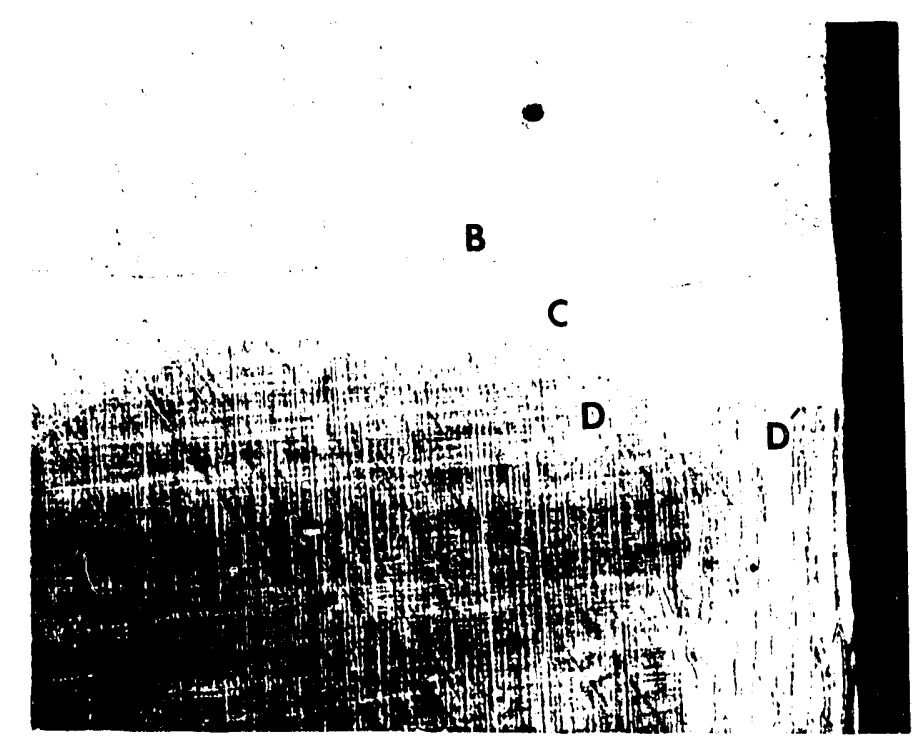

b)

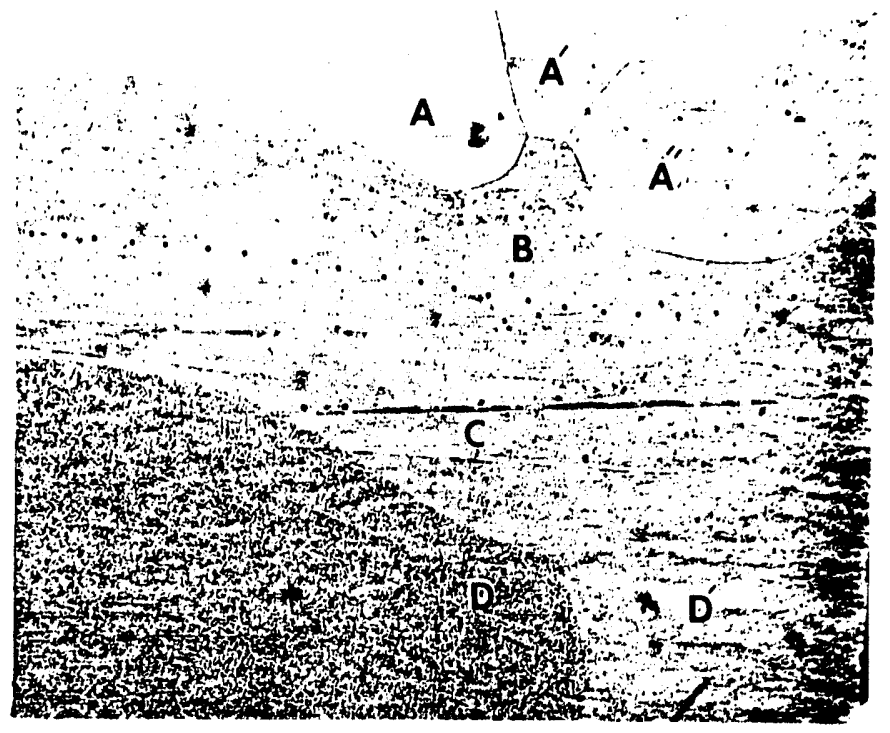

Figure 20. Optical micrograph a)before and b) after heat treatment in the sample of Exp.\# 64 at $1200^{\circ} \mathrm{C}$. Orientations of A-D positions were examined before and after heat-treatment. Dashed line on b) shows the locations of theoriginal interfaces \# I and II. a) $50 x$ and b) $100 x$ 
a)

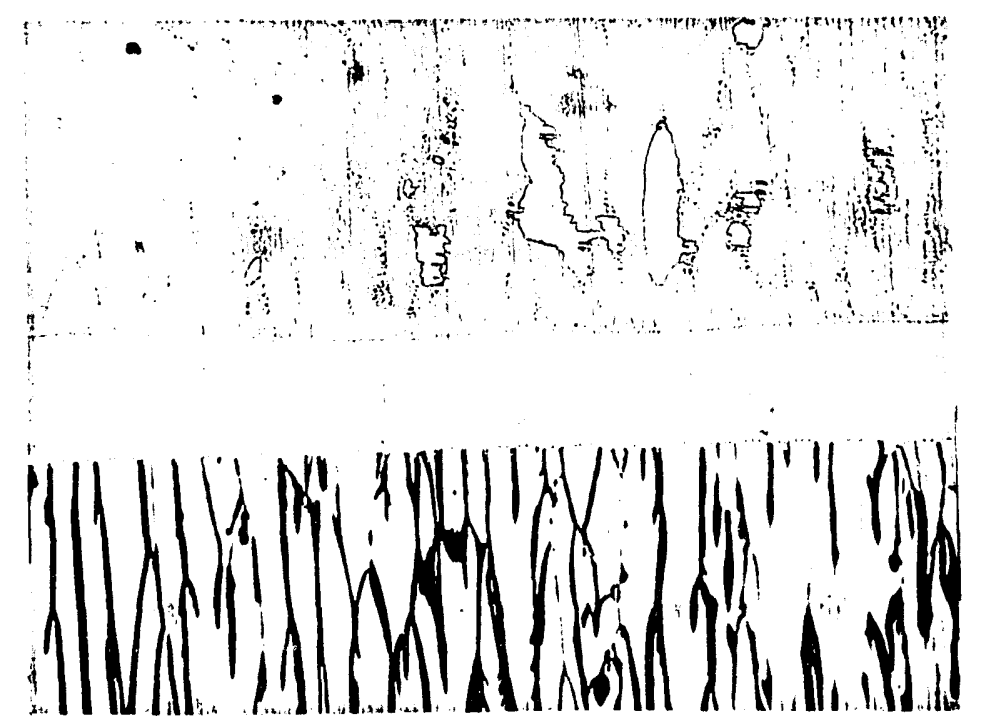

b)

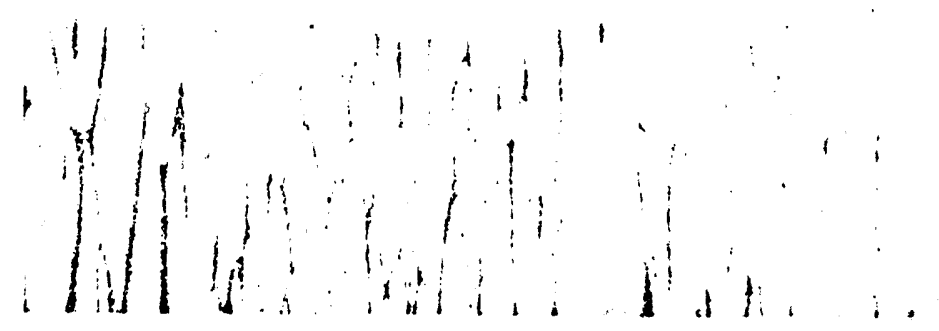

Figure 21. Optical micrographs a) before and b) after heat-treatment in the sample of Exp.\# 94. a) $100 x$, b) $100 x$ 
Janssen[2] has evaluated the mutual diffusion coefficient in the $\gamma^{\prime}$ phase and reports the following value: $\mathrm{D}=4 \operatorname{Exp}(-32200 / \mathrm{T}) \mathrm{cm}^{2} / \mathrm{s}$. The diffusion which is expected to occur away from the interfaces on heating may be modeled by assuming that a concentration of $\mathrm{C}_{0}$ above the concentration in the neighboring regions of the boundary is present at the boundary to a thickness of $h$ on either side of the boundary. The concentration at the boundary will decay with time according to the following solution of Fick's second law [3].

$$
\mathrm{C} / \mathrm{C}_{\mathrm{o}}=\operatorname{Erf}[\mathrm{h} /(2 \sqrt{\mathrm{D} t})]
$$

Taking values of $\mathrm{h}$ as $1 / 4,1 / 2$ and $1 \mu \mathrm{m}$, the predicted decay of the concentration in a 4 hour anneal as a function of the temperature of the anneal is shown in Fig. 22. The data of Table 3 indicate that the boundary has begun to disappear at $900^{\circ} \mathrm{C}$ after a $4 \mathrm{~h}$ anneal. For a $4 \mathrm{~h}$ anneal at $900{ }^{\circ} \mathrm{C}$ the plots shown in Fig. 22 indicate a reduction in concentration at the boundary region to $21 \%$ of the original $C_{0}$ value for $h=1 \mu \mathrm{m}$, and to 11 and $6 \%$ at $h=1 / 2$ and $1 / 4 \mu \mathrm{m}$. The estimated thickness of the segregation at the interfaces determined in the above section was 1 to $3 \mu \mathrm{m}$ or less, which would correspond to h values of $1 / 2$ to $1.5 \mu \mathrm{m}$.

In summary, the diffusion model clearly predicts that the boundary should not disappear at temperatures up to 650 to $700^{\circ} \mathrm{C}$, which agrees with the data. In order for the diffusion model to fully explain the beginning of the disappearance of the boundaries at $900^{\circ} \mathrm{C}$ requires that the boundaries maintain their visibility until the reduction of segregation is on the order of 10 to $20 \%$ of the original value. This requirement does not seem unreasonable and it is concluded that the heat treat experiments offer strong evidence that the presence of the boundaries is due to the segregation effect detected by the low voltage EDS experiments. 


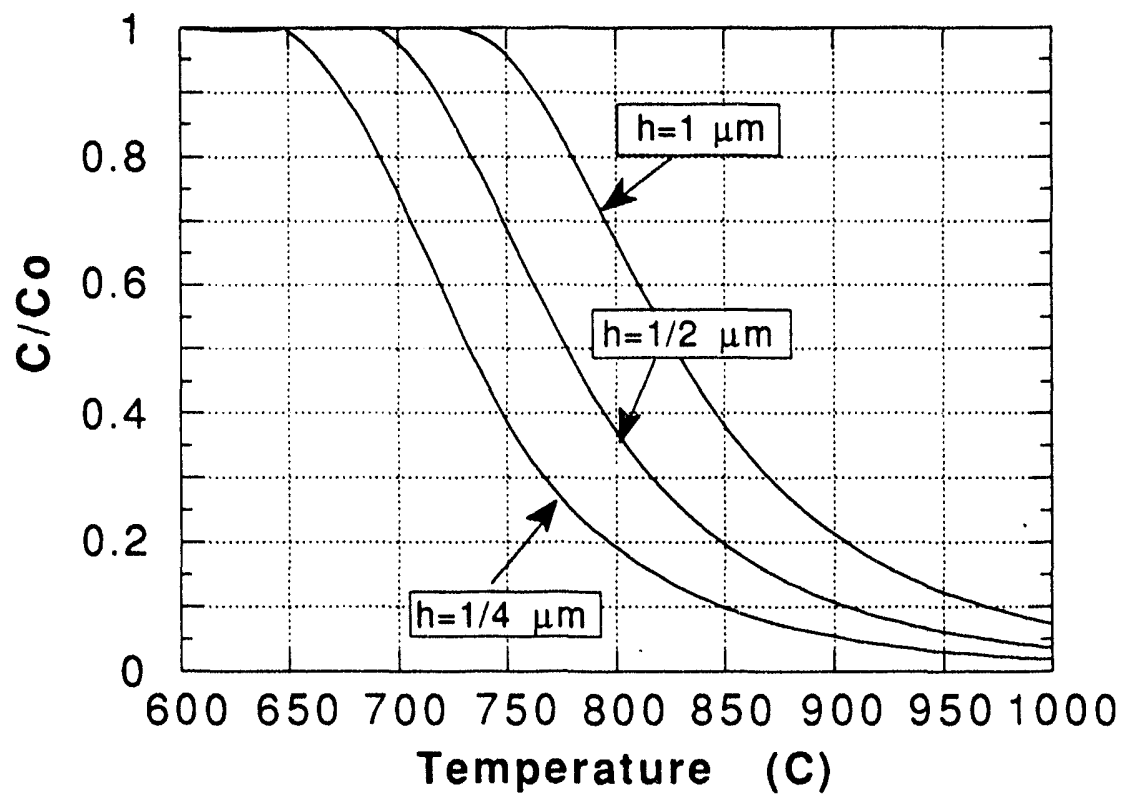

Figure 22. The predicted decay of the concentration in a 4 hour anneal as a function of the temperature of the anneal 


\section{DISCUSSION}

The quench rate control experiments lead to the conclusion that the original solid-liquid interface was the interface on the lower side of the $\gamma^{\prime}$ band (the interface \# I). From a series of experiments, a mechanism for formation of the $\gamma^{\prime}$ bands and the origin of mystery interfaces can be proposed with aid of Figure 4(b). Upon quenching the following sequence might occur: Step 1 - The $\gamma^{\prime}$ lower phase forms directly on the original solid-liquid interface in a sequence which starts at the outer wall and moves radially inward. Step 2 - The $\gamma^{\prime}$ lower band then grows longitudinally up the rod axis as single phase $\gamma^{\prime}$ until suddenly the $\beta$ phase begins to form. Then, the longitudinal growth continues as a cellular eutectic front. Step 3 - This eutectic growth developes into planar sheet arrays growing upward at several different orientations with each sheet having a eutectic microstructure. At the same time the liquid above the advancing solid-liquid interface nucleates solid in it, probably at the wall, and this solid grows radially inward with the cellular eutectic interface. These quenched liquid grains stop growth at the "UQL/Up growing solid" boundary when they meet with the upward moving eutectic front. Step 4 - If the quench is fast enough the $\beta$ phase at and above the top of the

$\gamma^{\prime}$ lower band does not decompose on cool down and therefore no $\gamma^{\prime} / \gamma^{\prime}$ interface \# II forms, but now a $\gamma^{\prime} /$ eutectic interface is present at the top of the $\gamma^{\prime}$ lower band (i.e., $\delta_{2}=0$ ). If the quench is not too fast (such as normal water quench of Figure 3(a)), the eutectic structure formed in Steps $\mathbf{2}$ and $\mathbf{3}$ transforms to the $\gamma^{\prime}$ phase during cooling down to room temperature. Therefore, the interface \# II is a $\gamma^{\prime} / \gamma^{\prime}$ boundary located at the point where the upward growing $\gamma^{\prime}$ lower band changed from single phase growth to eutectic growth involving the $\beta$ phase.

\section{Models for The $\gamma^{\prime}$ lower Band Formation}

An important question is how the $\gamma^{\prime}$ lower band forms upon the growing solid interface during the quench as proposed in Step 1. Three models might be suggested for formation of 
the $\gamma^{\prime}$ band, as shown in Figure 23. Model 1. The $\gamma^{\prime}$ nucleates at the $\gamma^{\prime}$ of the $\gamma+\gamma^{\prime}$ two phase region, which is near the outer wall, and grows laterally across the original interface in the radial direction without additional nucleation. Model 2.The $\gamma^{\prime}$ nucleates unifor mly along the growing $\gamma^{\prime}$ solid phase and gro'ws upward. Model 3. The $\gamma^{\prime}$ nucleates at cusps on the solid-liquid interface. Such cusps ocurr at the following possible locations: a. Liquid/wall/outer solid. This is the outside surface of the sample in contact with the wall of the container tube. b. Liquid $/ \gamma / \gamma^{\prime}$. These cusps occur whenever $\gamma+\gamma^{\prime}$ grow together into the liquid (annular regions of Figs. 3(a), 3(b), and 3(d), and entire interfaces of Figs. 3(c) amd 3(e)). c. Liquid $/ \gamma / \gamma$. These cusps occur when $\gamma / \gamma$ grain boundaries grow into the liquid (center regions of Figs. 3(d) and 24(a)). d.Liquid/ $\gamma$. Here there are no trijunctions but the cusps occur in the $\gamma$ growth front, as shown in Figure 25(b).

\section{The $y$ planar interface with an outer annular $y+y^{\prime}$ region}

Experiment 51 provided important information. It used the fastest quench (Ga-In) and was the only one which did not show the $\gamma^{\prime}$ lower band extending all the way to the center of the sample rod. Here the solid of the solidifying interface is pure $\gamma$ phase in the center of the rod with an outer annular ring of 2 phase $\gamma+\gamma^{\prime}$, see Figure 3(d). Figure 3(d) shows clearly that the $\gamma^{\prime}$ band formed initially at or very near the container wall and then grew radially inward toward the center of the rod. Figure 24 presents models for the mode of growth of the $\gamma^{\prime}$ lower band. In Model 3 of Figure 24(a) the $\gamma^{\prime}$ lower band nucleates first in the outer 2-phase annular region as discrete patches which grow together to form an initial outer band of $\gamma^{\prime}$. This would then be followed by additional nucleation of patches on the $\gamma$ surface at grain boundary junctions and at cusps in the $\gamma$ surface nearer the center of the sample. Figure 24(b) presents Model 1, where the outer $\gamma^{\prime}$ lower band nucleates first at the container wall and grows inward radially from that point. In either case there would be more longitudinal growth near the outer wall to account for the larger values of $\delta_{1}$ found there. It is not possible to determine the relative amount of radial 


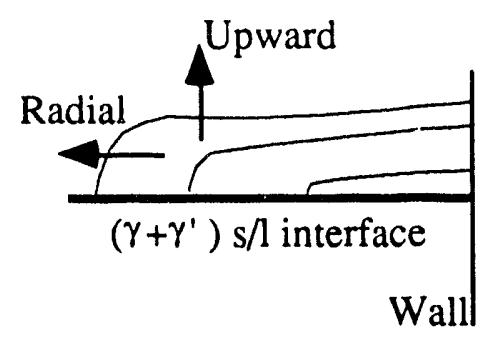

Model 1

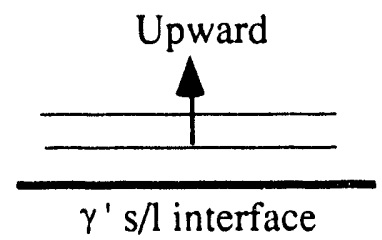

Model 2

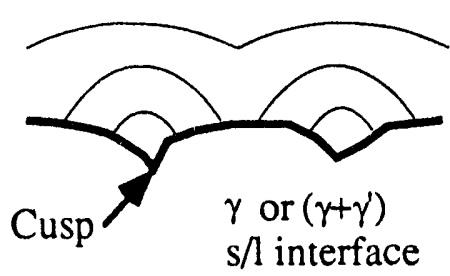

Model 3

Figure 23. Models to nucleate the $\gamma^{\prime}$ phase at the solid-liquid interface during quench procedure 
a)

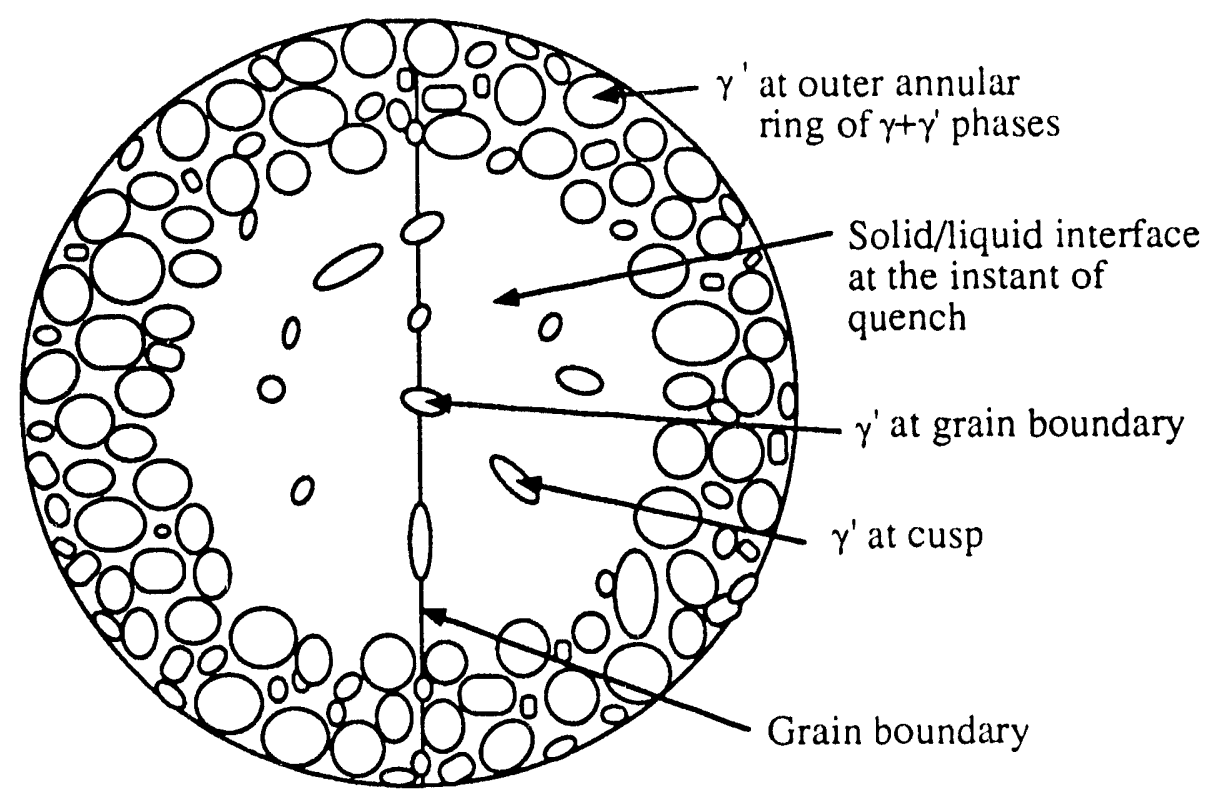

b)

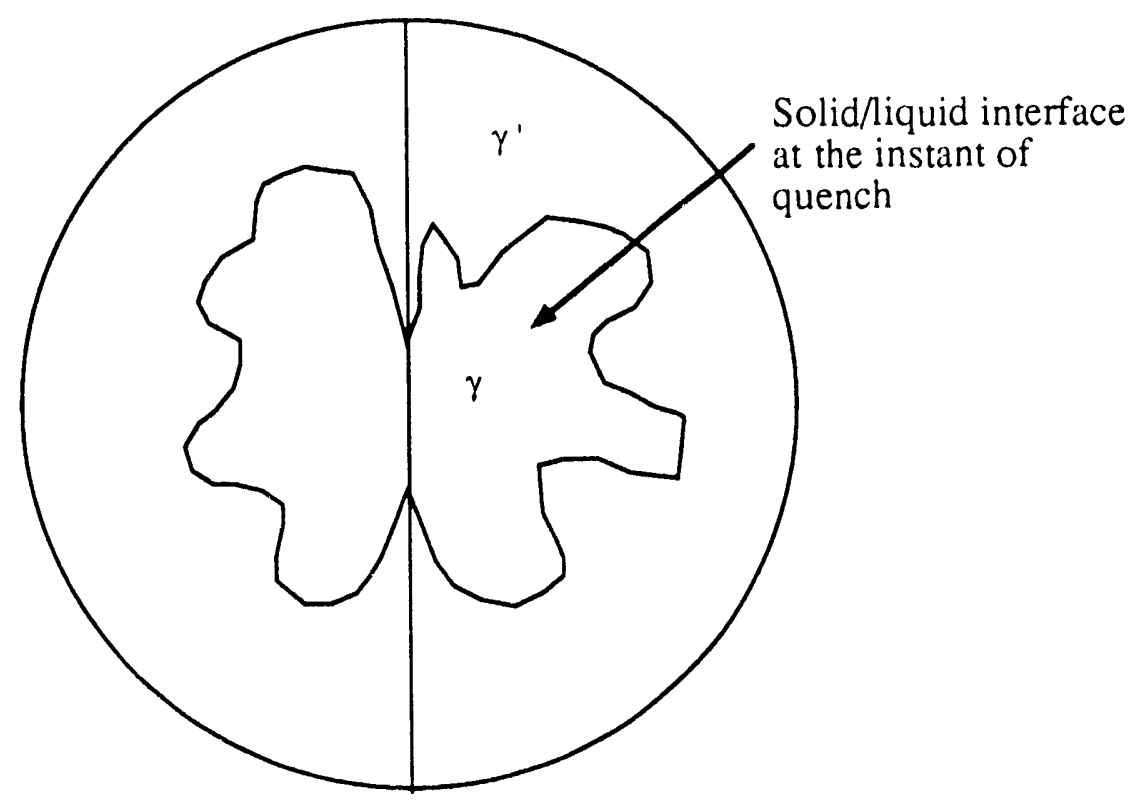

Figure 24. Schematic diagrams of the $y$ formation at the solid-liquid interface following the quench. (a) Model 3 and (b) Model 1 as transverse views 
a)

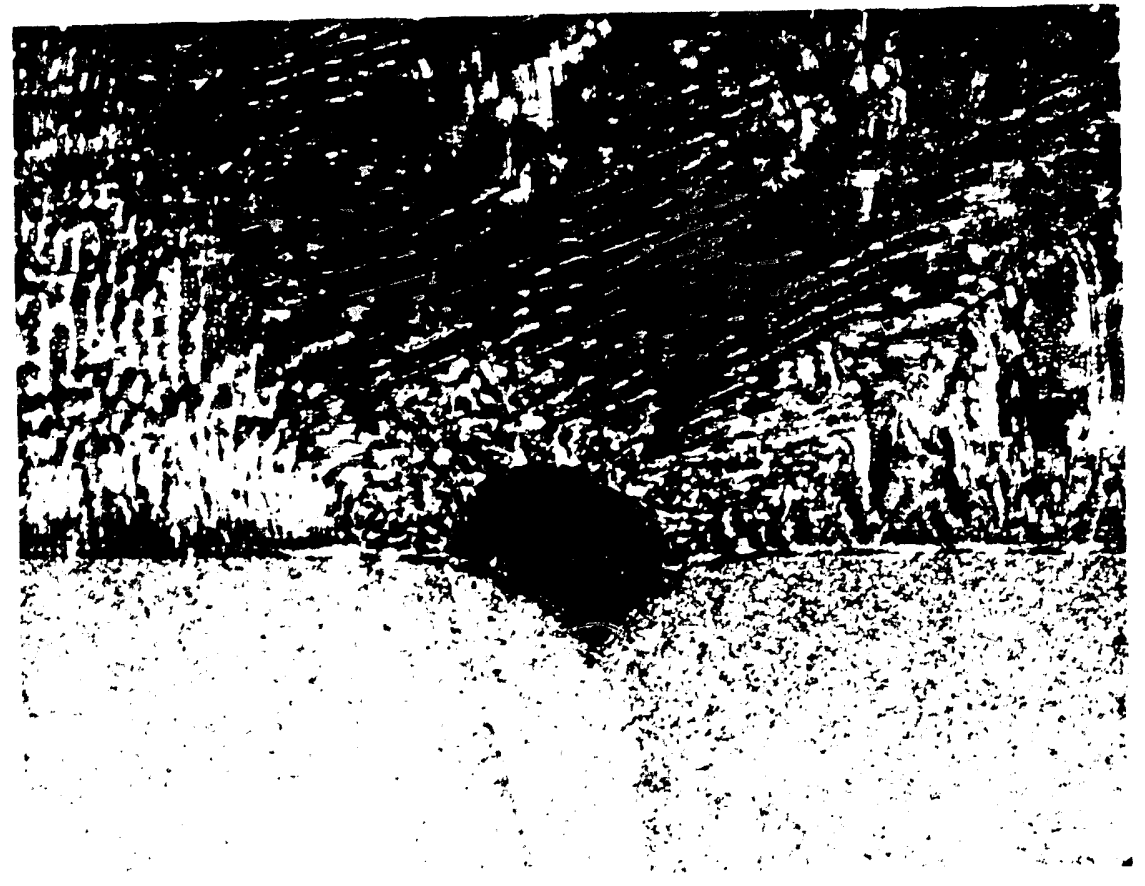

b)

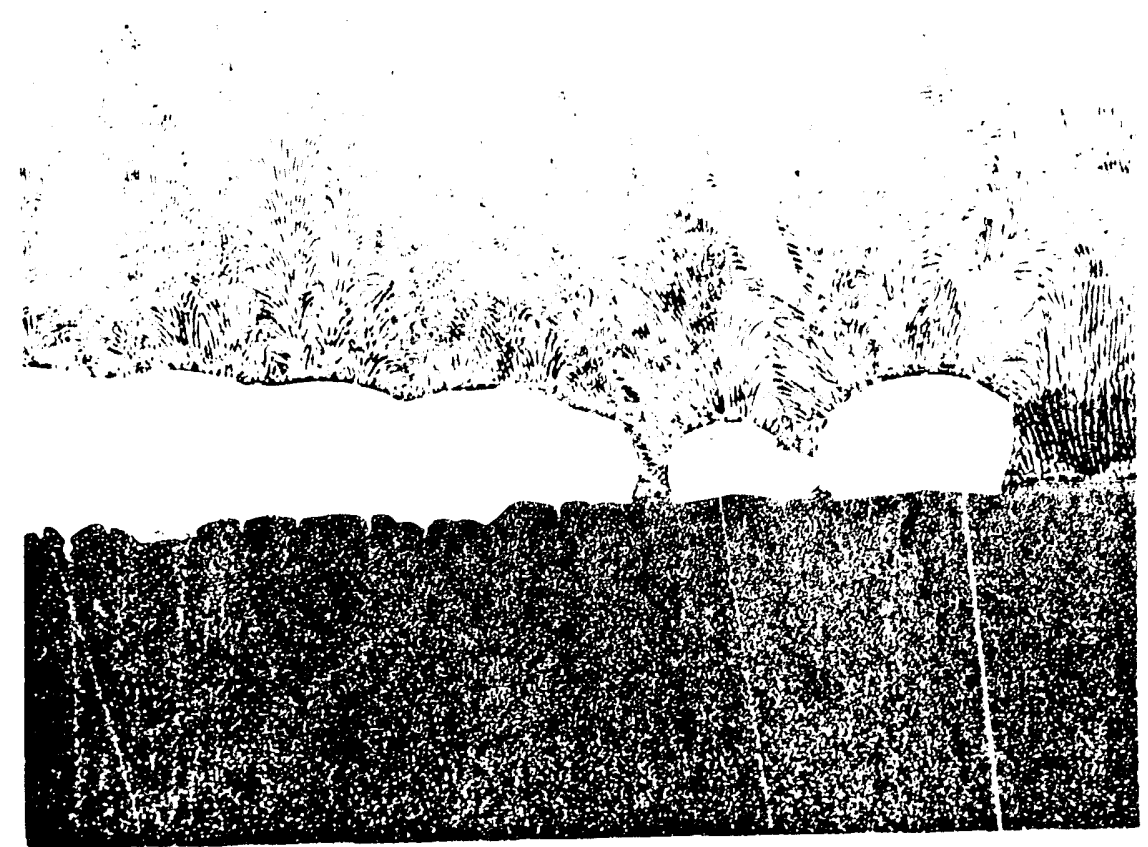

Figure 25. The nucleation of $\gamma^{\prime}$ at the $\gamma$ solid-liquid interface in fast quench of Ga-In (Exp. \# 511. a) At a grain boundary in the center region of Figure 3(d), 50)() DIC. b) At the cusps in the yplanar interface, 2()()$x$ 
growth, Model 1, versus cusp growth, Model 3, because the apparent radial growth seen in Figure 3(d) could be accomplished by a time sequence of nucleation at cusps. In other words, Model 3 could account for the results of Figure 3(d) if all growth of the $\gamma^{\prime}$ lower band occurred by nucleation at cusps sequenced in time such that fewer nucleations occured toward the center of the rod where the cooling rate was the slowest.

The crystallographic orientation of the band was compared to that of the $\gamma$ solid in Exp. \# 51 of Figure 3(d) by SACP in the SEM. There were two crystallographic orientations of the $\gamma$ at the solid-liquid interface with a vertical boundary separating them as seen on Figure 3(d). The left side and right side orientations of the $\gamma$ phase were different, but the orientations of the $\gamma^{\prime}$ lower band matched that of the $\gamma$ substrate on each side of the sample in Figure 3(d). The $\gamma^{\prime}$ in the 2-phase region at the edge of the sample also had the same orientation as the immediately neighboring $\gamma$. These results indicate that no matter where the $\gamma^{\prime}$ band nucleates it does so with epitaxy for the underlying solid $\gamma$ or $\gamma$ phase. Hence, although the crystallographic data prove that the $\gamma^{\prime}$ band must nucleate upon the growing $\gamma$ or $\gamma^{\prime}$ phases, it does not clearly distinguish between Model 1 and 3.

An important question has to do with the location of the solid-liquid interface at the instant of quench. In both Figures 26 and 5 one sees a planar $\gamma$ interface next to a cusped $\gamma$ interface. Consider first Figure 26. The right hand side of the apparent solid-liquid interface lies nearer the outer edge of the sample and it contains several cusps, whereas the left hand side has a planar $\gamma$ interface. One can make two arguments for the position of the $\gamma /$ liquid interface at the instant of quenching:

1) The interface was located essentially at the position of the $\gamma$ front seen in Figure 26.

2) The interface was located at the base of the cusps and during the quench the right hand side(in Figure 26) broke down into a cellular structure as it started to move upward. The cusps developed and the $\gamma^{\prime}$ nucleated in the cusps and stopped the upward growth of the cells. The center of the sample cooled slowly and therefore it did not break down into the cellular 


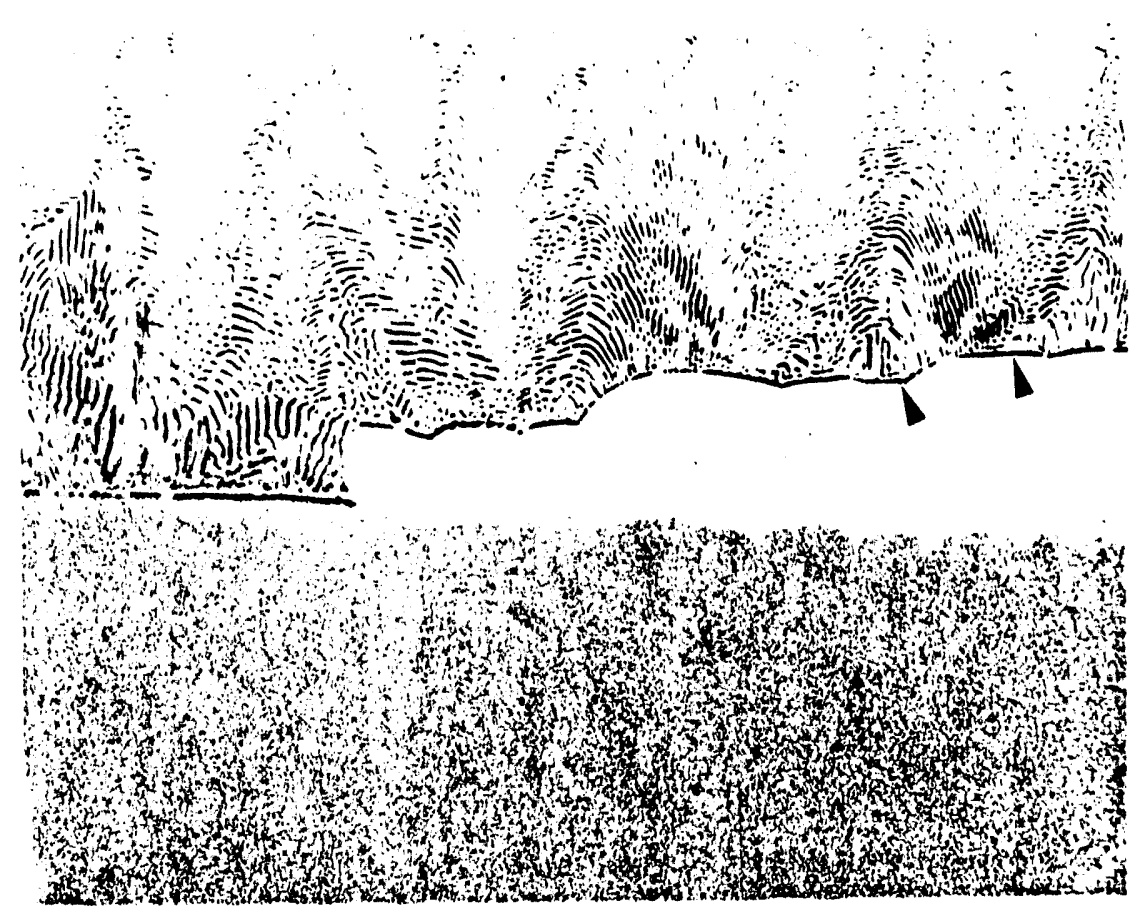

Figure 26. High magnigication in the center region of Figure 3(d). Left direction is to center and right direction is to edge. The $\gamma^{\prime}$ planar interface without cusps is shown in the center region. $250 \mathrm{x}$ 
structure with cusps. Therefore, it was able to grow upward further than the cusped regions before it was stopped by the formation of a film of $\beta$ phase. Explanation 2 seems logical and would require the $\gamma /$ liquid interface at the instant of quench to have been located at the base of the cusps and would mean that the cusps seen on Figure 26 were not present during solidification, but are an artifact of the quenching process.

The data of Figure 5 must also be considered. This case differs in two ways from Figure 26. The planar region here lies closer to the container wall, and the sample was quenched at a slower rate. For Figure 26 it was postulated that the reason the cusped region broke down into the cellular morphology was because it moved upward faster after the quench since it was closer to the quenched container wall. In Figure 5 however the planar region is closer to the container wall and it should have become cellular before the center region did. Also, the quench was slower with Figure 5 so that if the cusped regions were forming on quenching one might expect some differences in the cellular morphology and none is apparent. Another factor that needs to be considered is possible effects from radial composition changes. The depression of the $\gamma$ interface near the container wall indicates a higher $\mathrm{Al}$ content there. The higher Al content would give rise to more constitutional supercooling and a greater possibility of interface breakdown to cells in the outer regions. However, the cells appears only in the inner regions. Hence, these arguments all indicate that the cellls were not formed during the quench and were probably present along the $\gamma /$ liquid interface at the instant of quenching.

\section{The $y+y^{\prime}$ Planar Interface}

When the entire solid-liquid interface is a coupled $\gamma+\gamma^{\prime}$ planar morphology (Figures $3(\mathrm{c})$ and 3(e)), the $\gamma^{\prime}$ might nucleate from the growing $\gamma^{\prime}$ phase and grow upward by Model 2 and laterally by Model 1. The $\gamma^{\prime}$ might also nucleate at cusps of the solid-liquid interface by Model 3. The upward growth by Models $\mathbf{2}$ and $\mathbf{3}$ is thought to be dominant. This 
conclusion is suggested by results such as shown in Figure 27(a,b) whicn are high magnifications of the edge sides of Figure 3(c), where the near vertical grain boundaries in the $\gamma^{\prime}$ lower band have grown a little askew to the vertical direction.

\section{The $y^{\prime}$ Planar Interface}

For the case of the planar $\gamma^{\prime}$ interface the interface \# I is not seen in Marbles etch (Fig. 6(b)) and it is only after severe etching in HF that a very diffuse interface \# I is revealed. The interface \# II shows up clearly with the HF etch and again one sees that the longitudinal growth of the $\gamma^{\prime}$ band on quenching is significantly larger near the container wall (i.e., $\delta_{1 \mathrm{e}}>$ $\left.\delta_{1 \mathrm{c}}\right)$. As in the case of the $100 \% \gamma+\gamma^{\prime}$ interface, vertical grain boundaries in the $\gamma^{\prime}$ band suggest that the dominant growth mode is vertical, i.e., either Models 2 or 3. In addition, it is seen on Figure 27 that the vertical $\gamma^{\prime} / \gamma^{\prime}$ grain boundaries begin to slant radially inward at the location of interface \# I. Therefore, in the case of Figure $6(\mathrm{~b})$ one can infer that the original interface was located at the point where the vertical $\gamma^{\prime} / \gamma^{\prime}$ boundary on the right turns radially inward.

\section{The y Planar Interface}

When the solid-liquid interface was pure $\gamma$ planar where it was quenched at low solidification fractions, as shown in Figure 5(a,b) for Al 23 dlloy, the mystery interfaces were not apparently found. However, upon further etching and cureful examination of the pure $\gamma$ planar interface of the $\mathrm{Al} 23$ alloy, see Figure 28, it is seen that a $\gamma^{\prime}$ lower band has formed with a distinct cusped \# II interface. In this case it is unlikely that there was any lateral growth radially in from the wall because of the absence of $\gamma^{\prime}$ from the annular region. Apparently, the $\gamma^{\prime}$ lower band forms dominantly by nucleation at cusps as in Model 3 when no $\gamma^{\prime}$ phase is present in the solid. 
a)

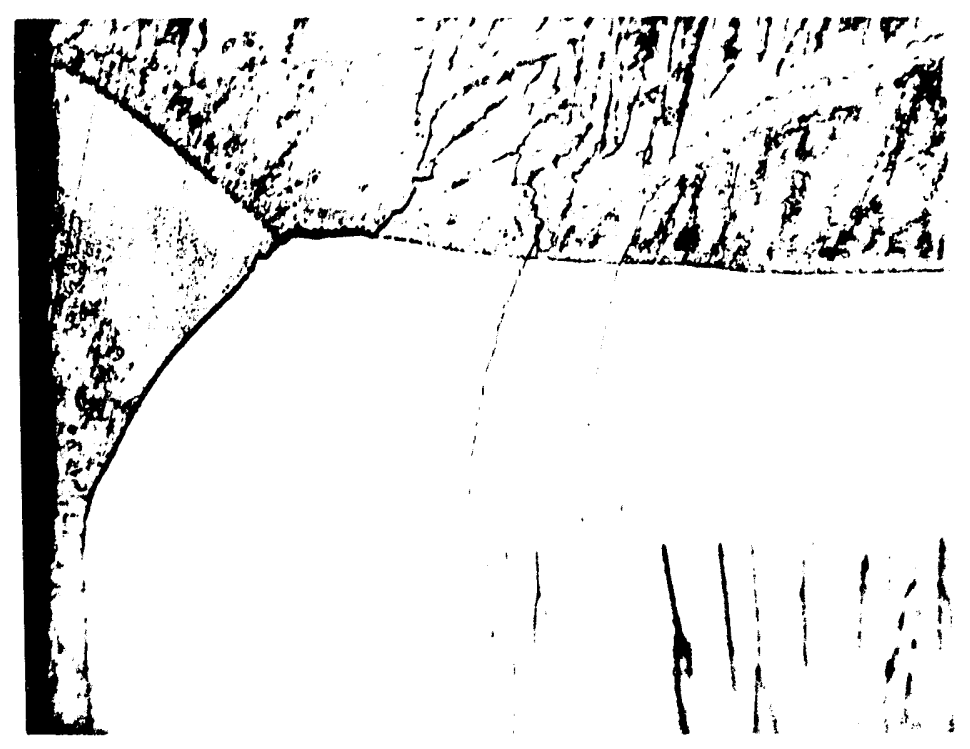

b)

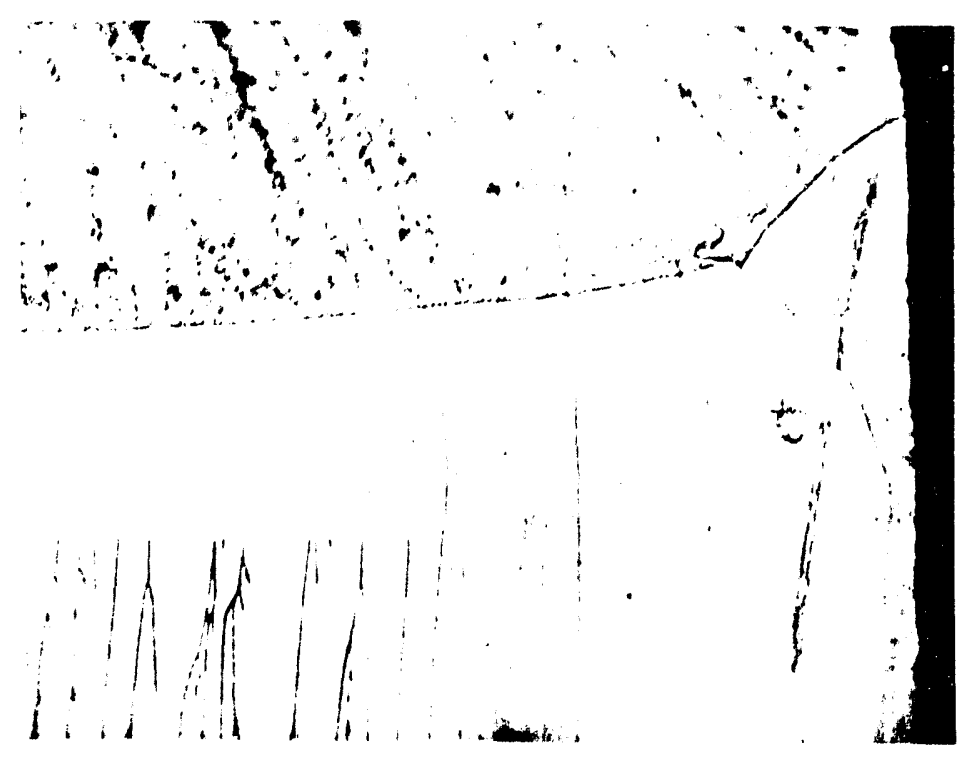

Figure 27. The $\gamma^{\prime}$ formation at the $\left(\gamma+\gamma^{\prime}\right)$ planar interface upon quenching. a) Left edge side and $b$ ) right edge side of Figure 3(c), 100x 


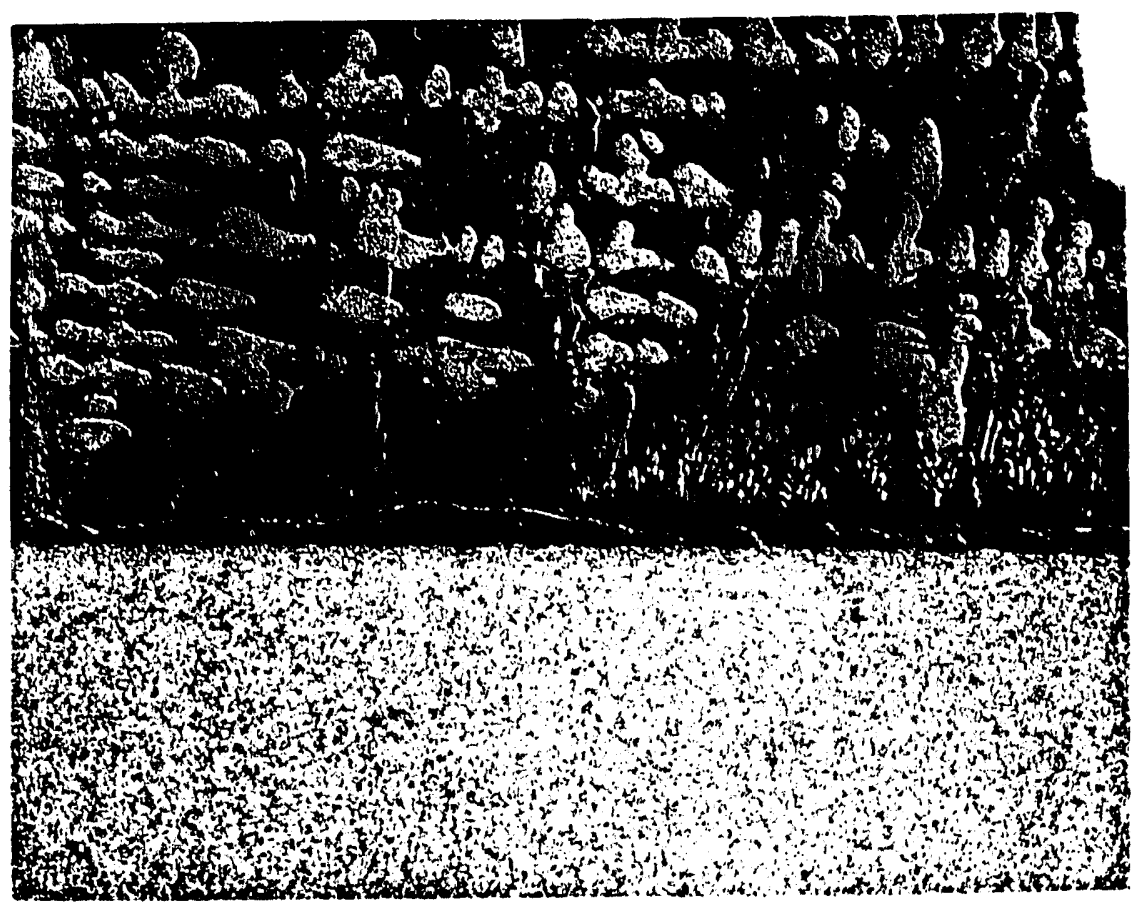

Figure 28. The $\gamma^{\prime}$ formation at the $\gamma$ planar solid-liquid interface upon quenching. This is a high magnification of Figure 5(b), 200x, DIC 
Formation of the Interface \# II

The experimental data presented above suggest the following model for formation of the mystery interface II. Upon quenching single phase $\gamma^{\prime}$ forms at the solid-liquid growth front and grows longitudinally with a cusped but flat interface as suggested by Steps 1 and 2 . Then, a layer of $\beta$ phase forms on the planar $\gamma^{\prime}$ front because an $\mathrm{Al}$ solute build-up occurs in front of the advancing $\gamma^{\prime}$ solid interface. This $\beta$ phase, which is rich in $\mathrm{Al}$, later may become the interface \# II. Additional upward growth occurs with a eutectic structure containing the $\beta$ phase, see Figures 25(b), 26, and right hans side of Figure 28. If the cooling rate is not so fast (i.e., normal water quench), upon cooling the $\beta$ phase in the fine eutectic above interface \# II is transformed into $\gamma^{\prime}$ phase having the same composition as the $\gamma^{\prime}$ immediately below the interface \# II. The layer of $\beta$ phase located at the interface \# II (such as that preserved by the fast $\mathrm{Ga}$-In quench, see arrows in Figure 26) also decomposes into $\gamma^{\prime}$ upon cooling, but produces a higher Al composition along the interface \# II location that is responsible for the appearence of the interface \# II.

If this model is correct, one would expect that as the $\gamma^{\prime}$ lower band grew longitudinally upward its $\mathrm{Al}$ composition would increase due to solute build-up in the adjacent liquid lying above it. EDS analysis shows that the composition increases across the $\gamma^{\prime}$ lower band, as expected, see Figure 15. This resuii was confirmed in three other samples examined with EDS in the SEM and TEM. These results support the idea that the interface \# II results because of the nucleation of a eutectic structure containing the $\beta$ phase after growth of the $\gamma^{\prime}$ lowcr band.

Formation of The $\gamma_{\text {upper }}^{\prime}$ Band and The $\gamma^{\prime}$ Polycristalline Region

As the eutectic grows upward from the $\beta$ phase at the top of the $\gamma^{\prime}$ lower band, the growth front developes into planar arrays of parallel sheets having eutectic microstructures. These sheets appears to grow along specific crystallographic directions (see Figures 3(b, c, d)) suggesting that they might be the initial form of a dendritic structure. However, since there is 
no evidence of side branch formation these structures will be called 2-phase cells. These cellular eutectic sheets stop growth when they meet with the upper quenched liquid (UQL) at the "UQL/Up growing solid" boundary. Figure 29(a) shows the microstructures of the UQL, the up growing solid, and their boundary at the fast quench of Ga-In, where all the sheets display eutectic structures. The UQL appears to grow radially inward also as cellular eutectic sheets. In order to confirm that the cellular eutectic was really a sheet morphology, a transverse section of the UQL and up growing solid regions was observed. The transverse view also showed the same sheet morphology as seen in Figure 29(a) and therefore the cellular eutectic must be the sheet morphology.

The $\gamma_{\text {upper }}^{\prime}$ band, the elongated $\gamma^{\prime}$ polycrystalline region, and the UQL are all initially eutectic structures before solid state transformation on cooling. The eutectic of the $\gamma^{\prime}$ upper band is the same crystallographic orientation with the $\gamma^{\prime}$ lower band, but the cellular eutectic sheets of the $\gamma$ ' polycrystalline and UQL regions have several different orientations. Figures 29 and 30 show good evidence that the $\gamma^{\prime}$ phases of these three regions is transformed from the eutectic. Figures 29(a) shows the UQL/Up growing solid boundary illustrating that solidification has occurred by growth of cellular eutectic sheets in both regions. The Ga-In quench was fast enough to preserve some $\beta$ phase at most locations. This cellular eutectic sheet structure transforms to the single phase elongated $\gamma^{\prime}$ polycrystalline region at the normal water quench as shown in Figure 29(b). Figure 30 provides additional informations about the mystery interfaces as well as about the transformation of the eutectic to the $\gamma$ single phase. At the relatively slow quench rates of normal water quench the $\gamma_{\text {upper }}^{\prime}$ band is the $\gamma^{\prime}$ single phase(see Figure 3(a)) but a trace of eutectic structure remains in Figure 30. The eutectic looks to be transformed to $\gamma$ single phase because even though the eutectic microstructure appears, no $\beta$ phase remains (the $\beta$ phase is etched black). Also, no evidence of $\beta$ phase was observed in the $\gamma_{\text {upper }}$ band in the TEM studies on water quenched samples The trace of eutectic microstructure seen in Figure 30 is thought to be the result of the composition variation in the 

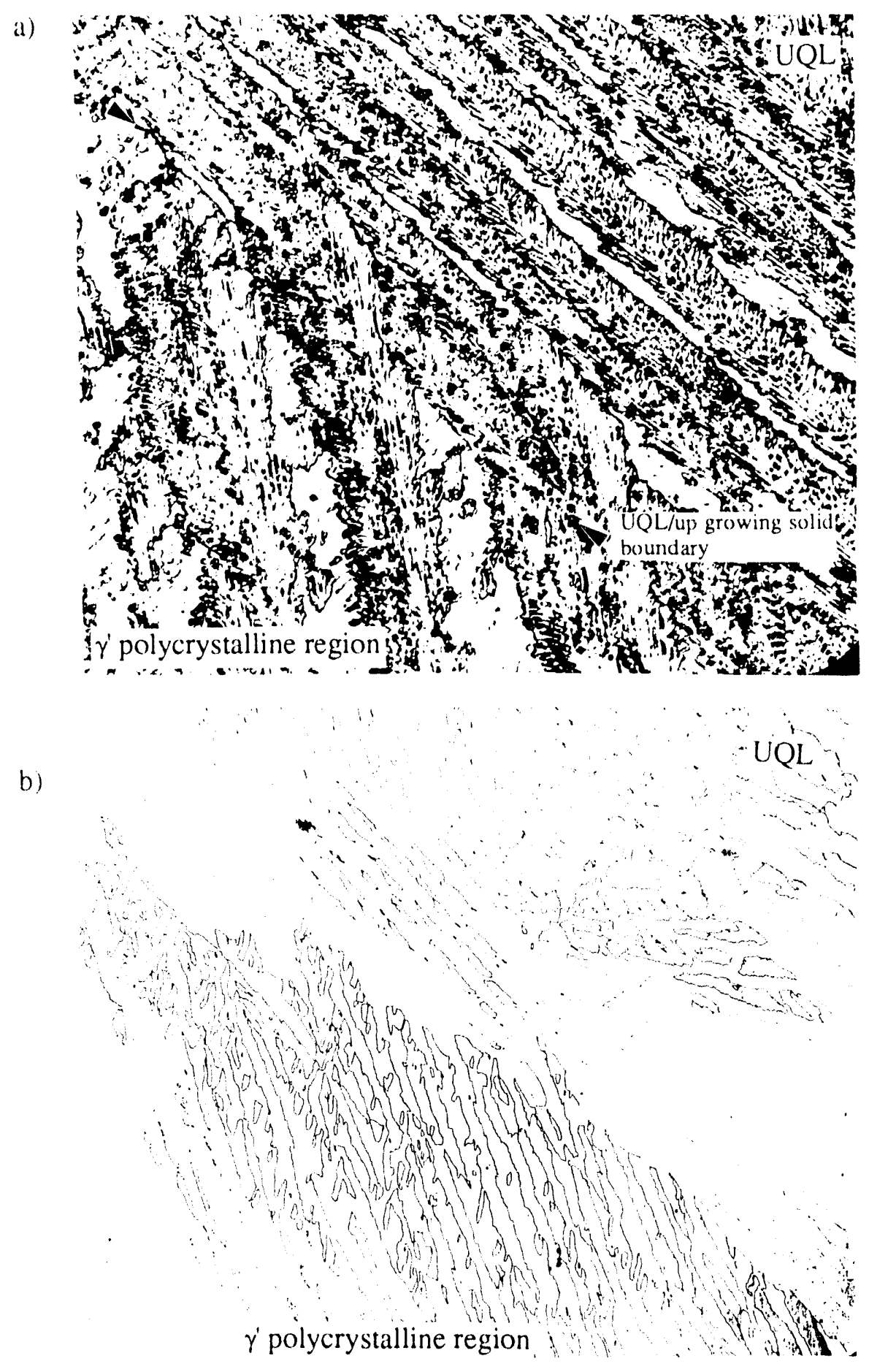

Figure 29. The microstructures of polycrystalline and UQL regions a) at fast quench of Ga-

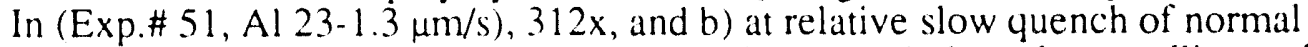

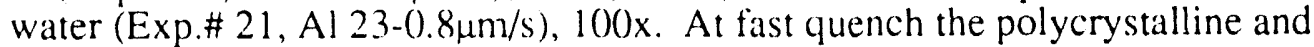
UQL region consist of the planar arrays of parallel sheets having eutectic microstructures 


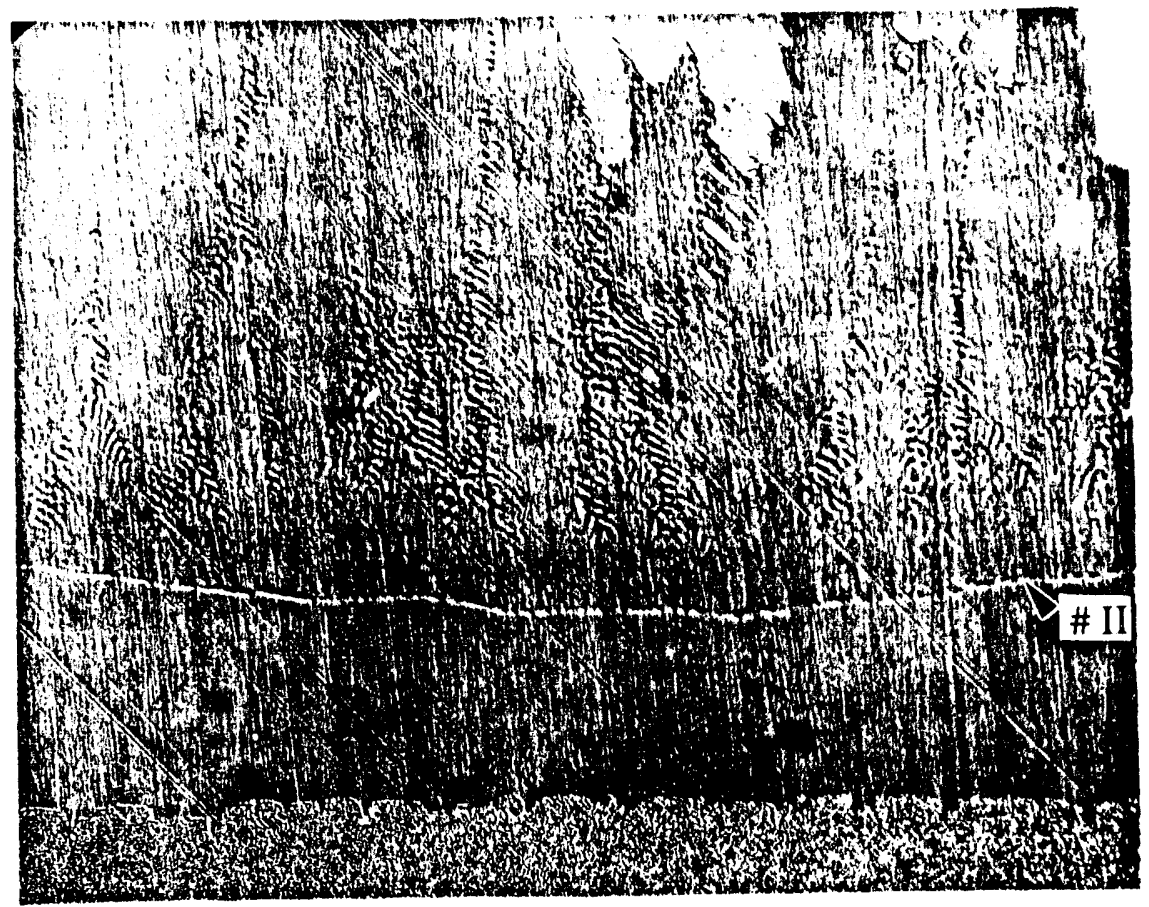

Figure 30. A trace of the eutectic in the $\gamma_{\text {upper }}$ band in Exp. \#21(Al 23-0.8-water Q). This figure is a high magnification in the $\gamma_{\text {upper }}^{\prime}$ band region of Figure 3(a). 250x, DIC 
$\gamma^{\prime}$ phase. The fact that the composition variation is able to produce an observable microstructural variation is additional evidence that mystery interfaces \# I and \# II are due to composition variations.

When the quench rates are increased, the $\delta 2$ values are reduced, as shown in Table 2. The $\delta 2$ region(the $\gamma^{\prime}$ upper band) is suppressed at such a fast quench of Ga-In. At slow quenches larger $\delta_{2}$ values could be obtained by solid state transformation. The cooling rate to room temperature of the up and ice-water quench must be faster than that of the normal drop quench because the $\delta 2$ regions are zero in the up quench or close to zero in the ice-water quench. At very slow quench of the air, recrystallization might also occur on cooling, as shown in Figure 3(e).

In conclusion, the $\gamma^{\prime}$ lower band ( $\delta_{1}$ region) is expected to be formed early in the quench and the $\gamma_{\text {upper }}$ band ( $\delta_{2}$ region) must be formed later by solid state transformation during cool down.

\section{Formation of the Interface \# I}

A clear interface \# I was observed for coupled growth of the $\gamma+\gamma^{\prime}$ phases, such as in the annular region for Exp. 21, shown in Fig. 4(a). In addition, similar phenomenon was sometimes found for eutectic growth. It was not found at the $y^{\prime}$ phase of the eutectic in the two phase eutectic growth front of $\gamma^{\prime}+\beta$. But, it always occured at three phase eutectic of $\left(\gamma+\gamma^{\prime}+\beta\right)$, as shown in Figure 31 (The eutectic structure will be discussed in Paper IV). This result indicates that in order for the interface \# I to appear the presence of at least some $\gamma$ phase is required at the solid-liquid interface.

The EDS result found an AI depletion at the interfice \# I in the coupled $\gamma+\gamma^{\prime}$ structure, as shown in Figure 17(a). The Al was also found to be depleted at the interface \# I in the three phase eutectic. The thickness of an Al depletion was less than $1 \mu \mathrm{m}$. The models of the formation of the $\gamma^{\prime}$ band show that the interface \# I is formed by lateral and upward growth of 

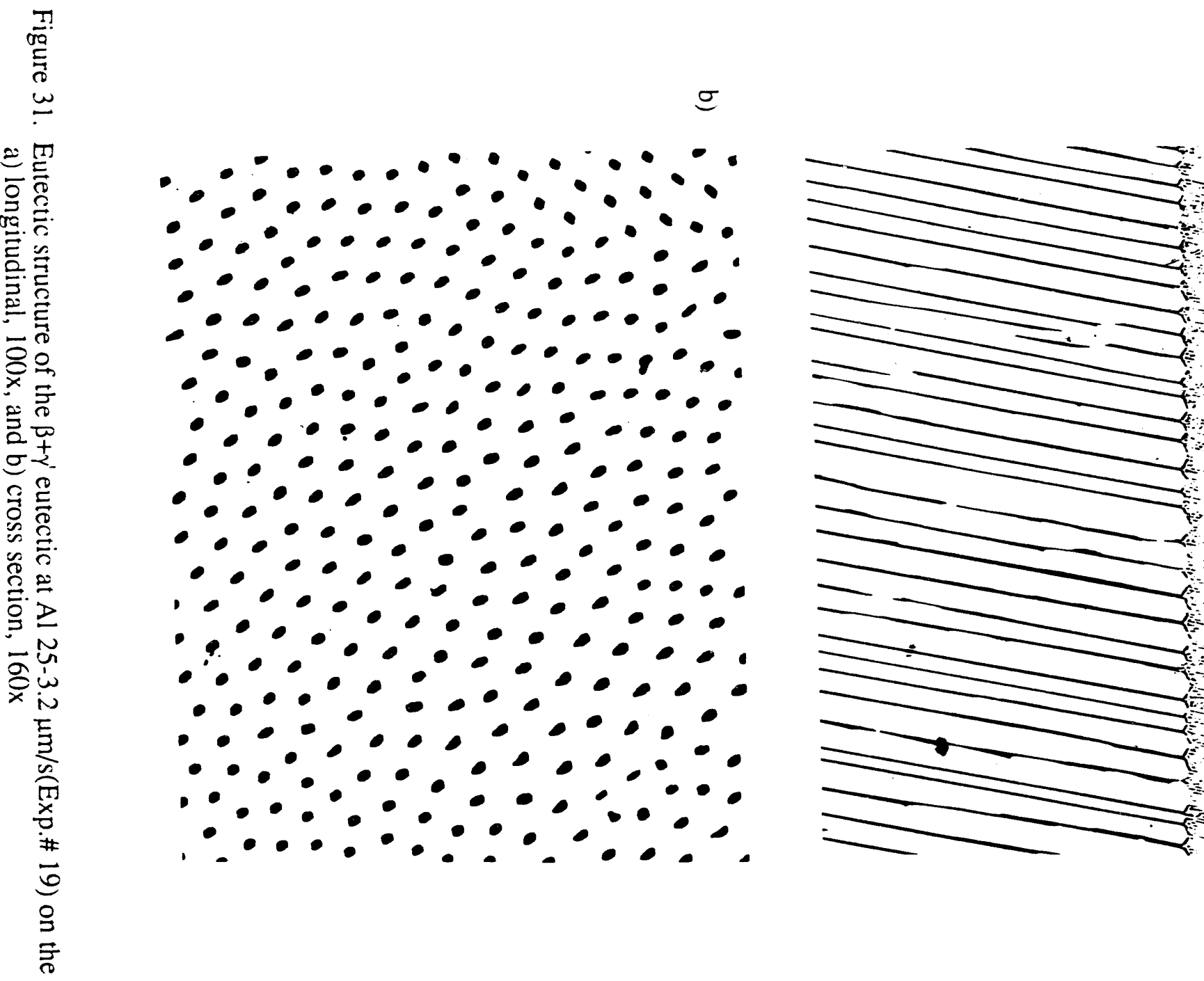
c)

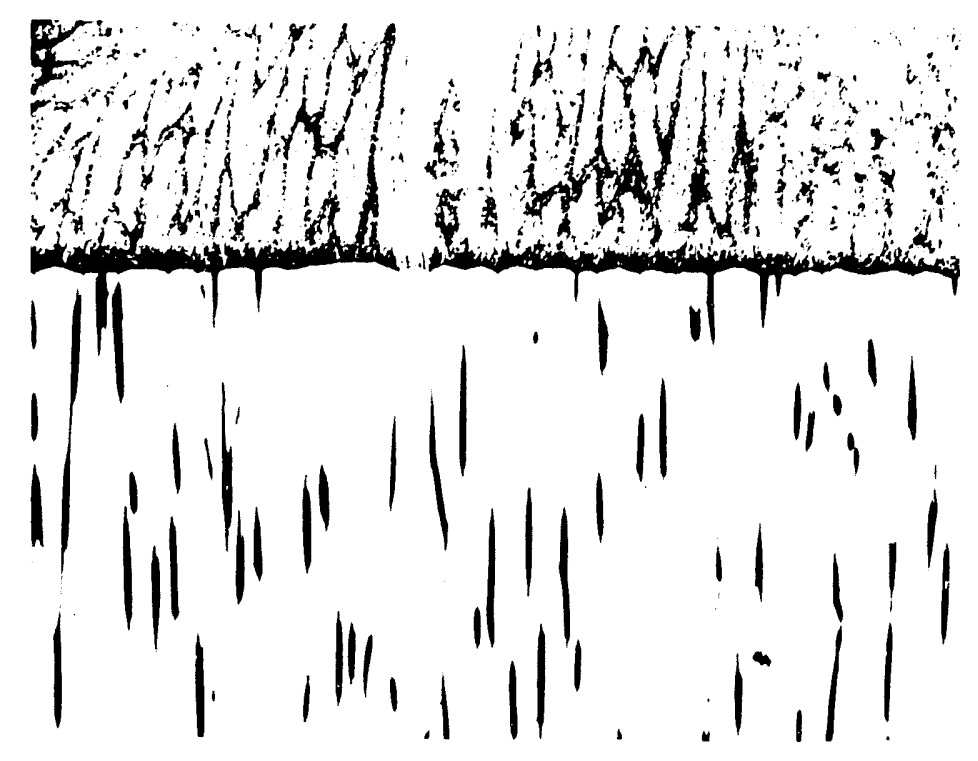

d)

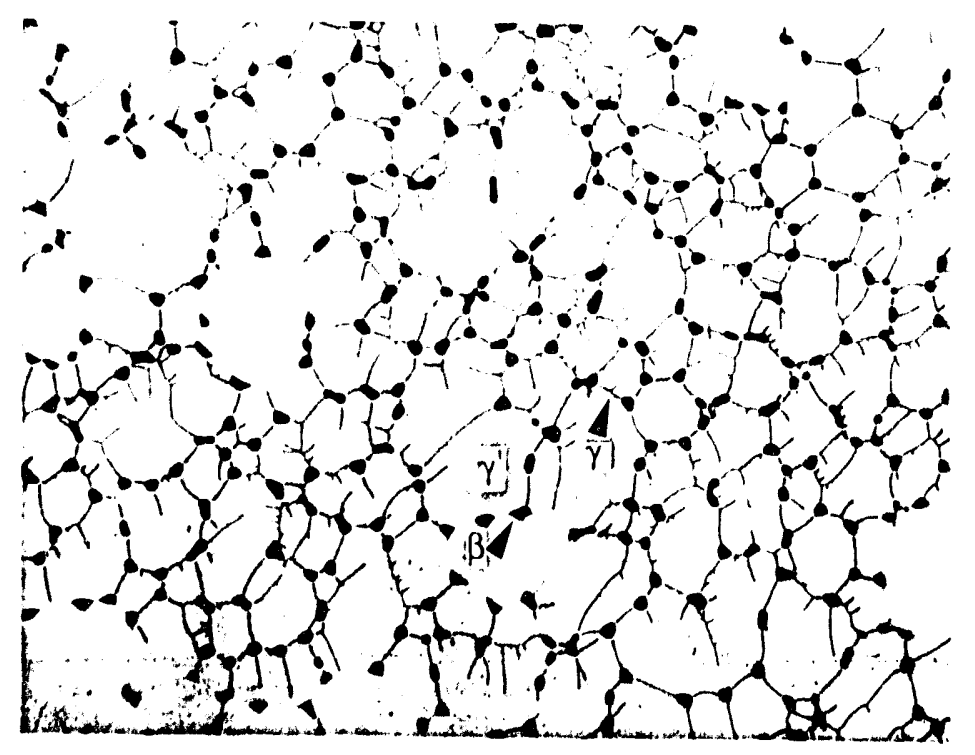

Figure 31. (continued) The $\beta+\gamma+\gamma$ eutectic at $\mathrm{Al} 24.5-3.2 \mu \mathrm{m} / \mathrm{s}(\operatorname{Exp} . \# 59)$ on the $\mathrm{c})$ longitudinal view, $5(\mathrm{x}$, and d) transverse view, 10() $\mathrm{x}$ 
a new thin band of $\gamma^{\prime}$ across a pre-existing liquid $/ \gamma^{\prime}$ interface. Follnwing is a proposed model for the formation of mystery interface \#I: First, a very thin layer of disordered $\gamma$ solid nucleates at the $\gamma / \gamma^{\prime} /$ liquid trijunctions and rapidly spreads laterally over the $\gamma^{\prime}$ phase. This layer will be low in $\mathrm{Al}$ as is characteristic of the disordered $\gamma$ phase. Second, upward growth from this thin $\gamma$ layer is continued by solidification of the ordered $\gamma^{\prime}$ phase with its higher $\mathrm{Al}$ composition. During cool down the original thin $\gamma$ layer transforms to $\gamma$ unless the cooling rate is very fast. The idea of lateral growth of $\gamma$ phase could be deduced by the results that there was no interface \# I on $100 \% \gamma^{\prime}$ planar interfaces but the Al depleted \# I interfaces were found when $\gamma^{\prime} / \gamma /$ liquid trijunctions were present, such as with the $\gamma+\gamma^{\prime}$ and $\gamma+\gamma^{\prime}+\beta$ interface. In addition, this idea is supported by the fastest quench(Ga-In) of Exp. 51, as shown in Figure 32 , where it appears that untransformed $\gamma$ phase is present along the interface \# I, see arrow.

In the $\gamma+\gamma^{\prime}+\beta$ eutectic interface of Figure 31(c), the $\gamma^{\prime}$ band is thickest at the center line of the $\gamma^{\prime}$ lamellae, even though the initial thin $\gamma$ layer must have reached this center line last. One can account for the thicker $\gamma^{\prime}$ layer at the centerline by considering the effect of $\beta$ phase growth on quenching. It appears that the $\beta$ phase propagates rapidly into the liquid upon quenching. It seems likely that upon quenching the thickness of the $\gamma^{\prime}$ band formed is controlled by $\beta$ growth as may be explained with Figure 33. The schematic diagram of Figure 33 shows that the $\beta$ grows laterally outward and cuts off the longitudinal growth of the $\gamma^{\prime}$ band. Since the $\beta$ reaches the centerline of the $\gamma^{\prime}$ lamellae last the $\gamma^{\prime}$ band ends up thickest at the center line.

It was initially thought that perhaps the appearence of the interfaces \#I and \#II might require specific orientations of the FCC phase. For example, in Part II it was shown that the etching caracteristics of $\gamma^{\prime}$ depends strongly on the crystallographic orientation. Therfore, the orientation dependence of the appearence of the interface \# I was examined in the $\left(\gamma+\gamma^{\prime}\right)$ coupled and $\left(\gamma+\gamma^{\prime}+\beta\right)$ eutectic structures, where the interface \# I was clearly shown, and in the $\gamma^{\prime}$ planar and $\left(\gamma^{\prime}+\beta\right)$ eutectic structures, where the interface \# I was not shown, by SACP in the 


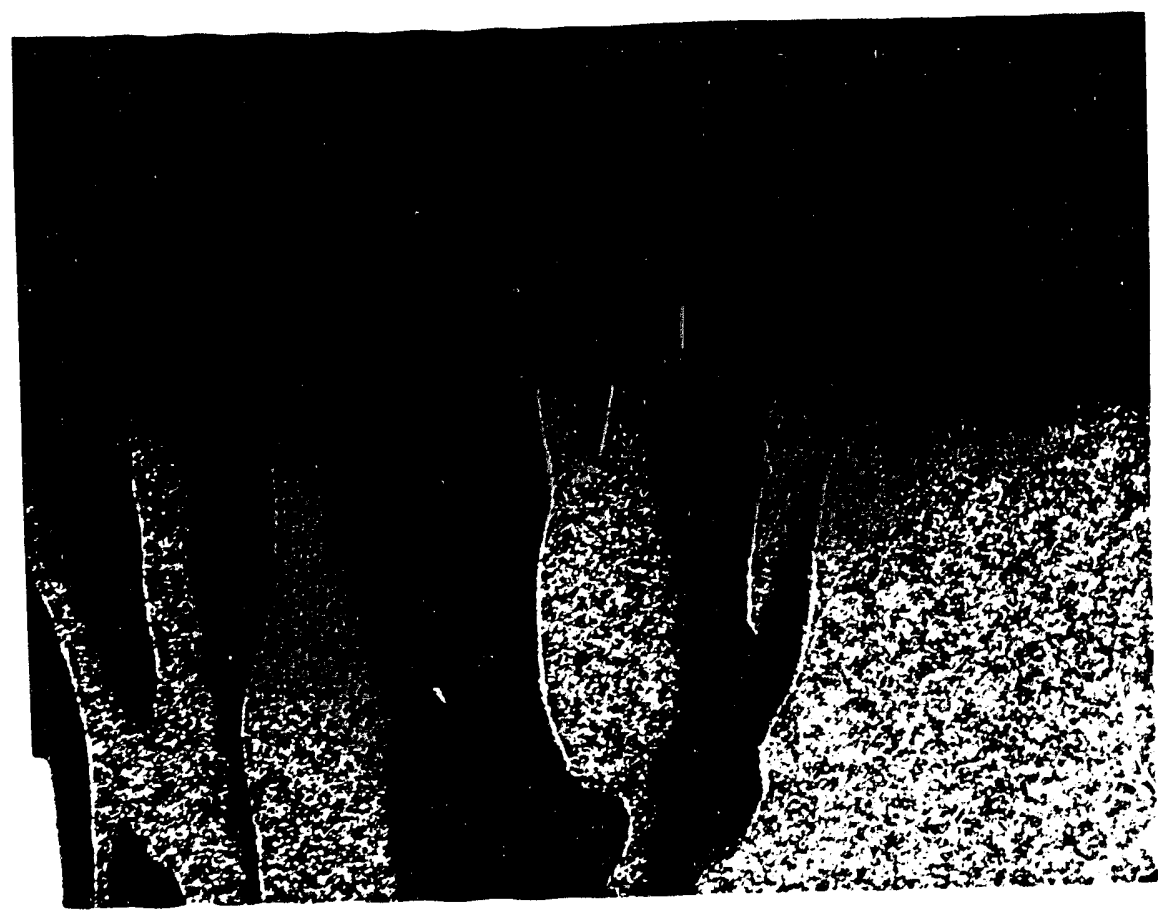

Figure 32. The coupled $\gamma+\gamma$ ' solid-liquid interface in Ga-In quench (Exp. \# 51). Fine $\gamma$ phases(arrow) line up in front of $\gamma^{\prime}$ phase, 500x, DIC 


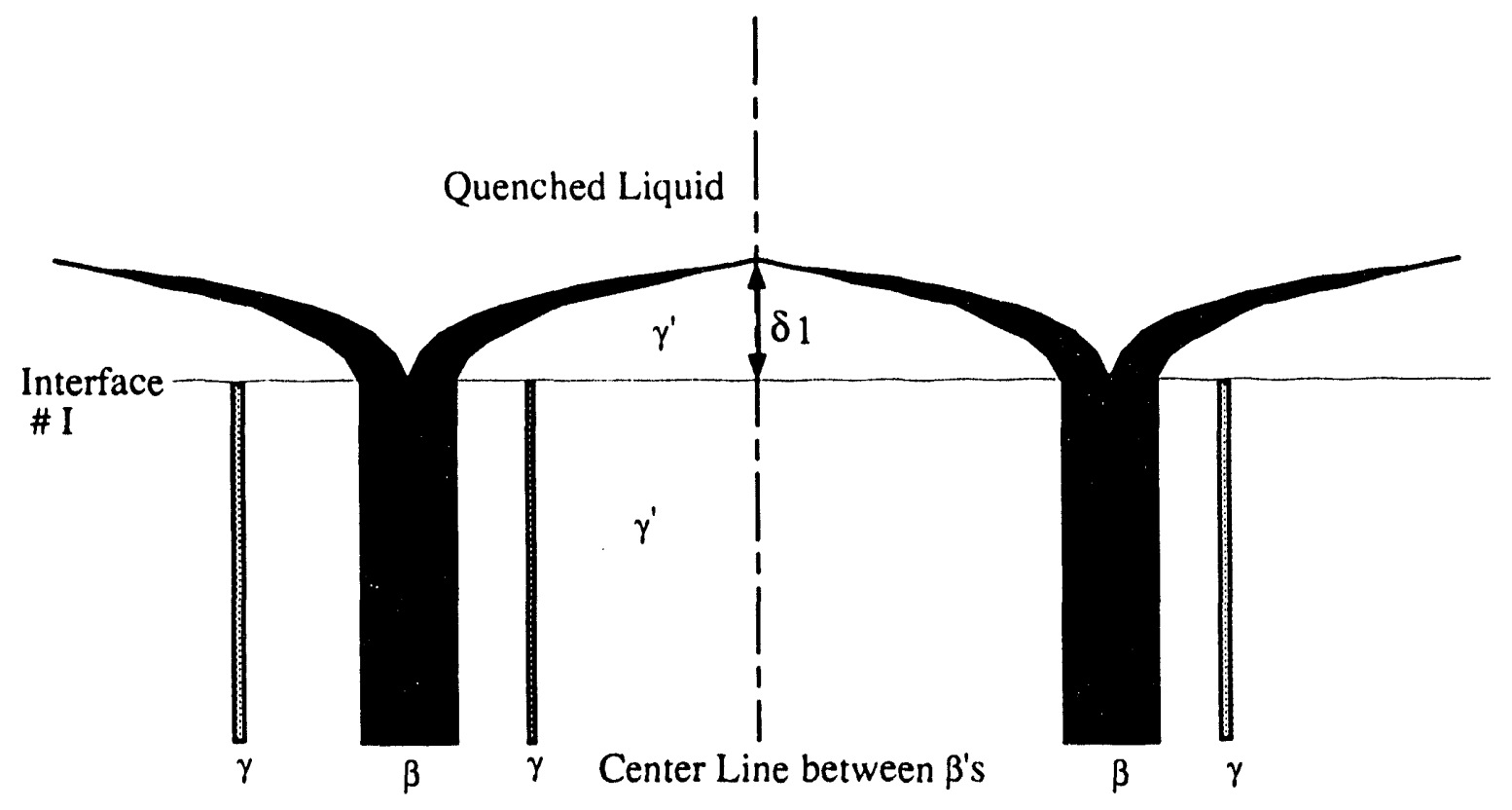

Figure 33. Schematic diagram of the $\left(\gamma^{\prime}+\gamma+\beta\right)$ eutectic growth front of Figure 31(c) 
SEM. Exp. \# $5 \mathrm{I}(\mathrm{Al} 23-1.3 \mu \mathrm{m} / \mathrm{s})$ and Exp. \# 56(Al 24-3.2 $\mu \mathrm{m} / \mathrm{s})$ were used for the $\gamma+\gamma^{\prime}$ coupled structure, Exp.\# 59(Al $24.5-3.2 \mu \mathrm{m} / \mathrm{s})$ for the $\left(\gamma^{\prime}+\gamma+\beta\right)$, Exp. \# (Al 25-6.3 $\left.\mu \mathrm{m} / \mathrm{s}\right)$ for the $\left(\gamma^{\prime}+\beta\right)$ eutectic, and Exp. \# 57(Al 24-1.5 $\left.\mu \mathrm{m} / \mathrm{s}\right)$ for the $\gamma^{\prime}$ planar. The crystallographic orientations were examined on the surfaces of longitudinal sections. Figure 34 shows that there is no special orientation relationship between existance and absence of a interface \# I.

The above experiments indicate that the mystery interfaces of \# I and \# II were formed by sharp composition changes extending over less than 1-3 $\mu \mathrm{m}$. Such a change in composition would produce only a gradual change in the structure of the lattice. That could be the reason no boundary or fringe patterns were found at the mystery boundaries in the TEM. 


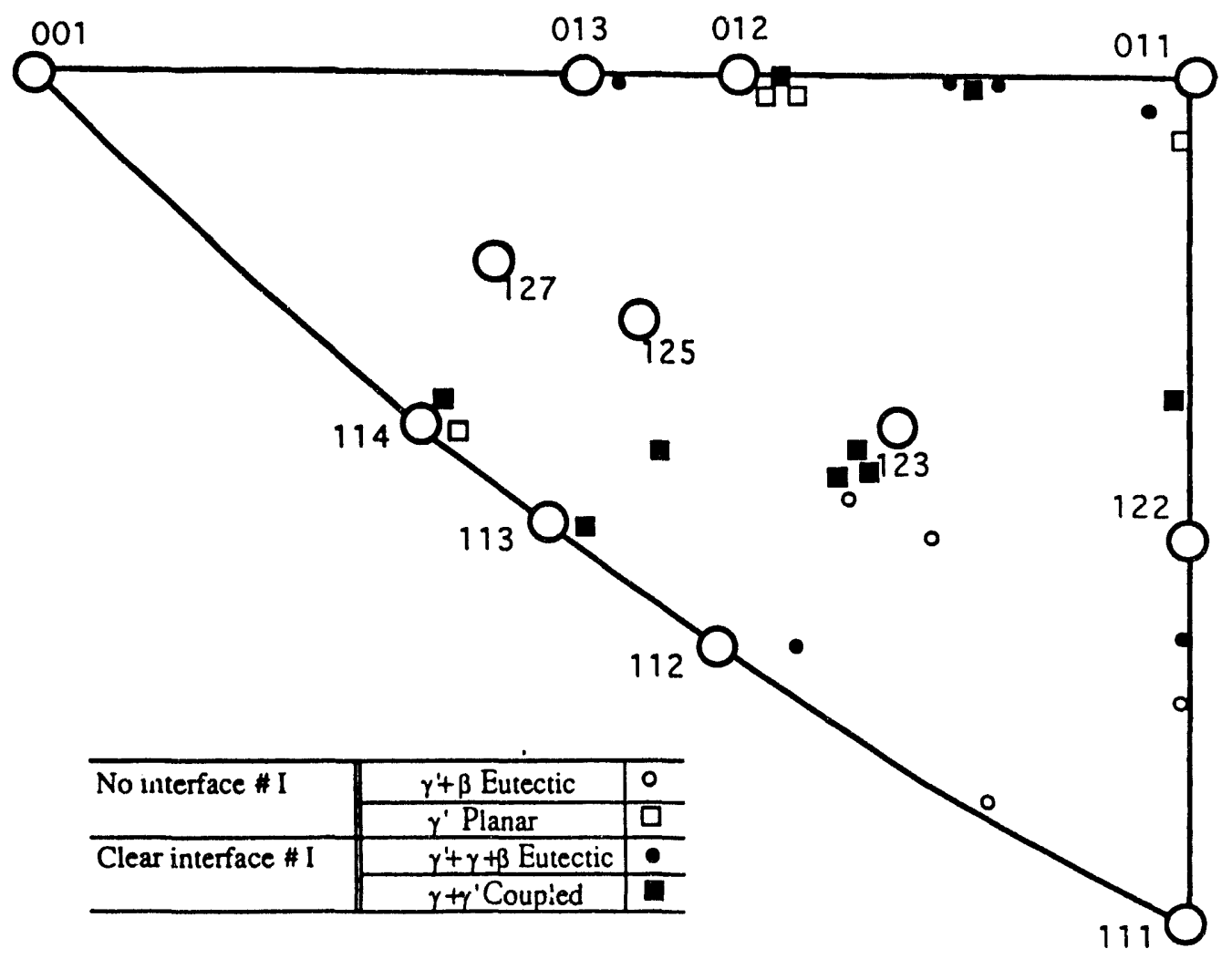

Figure 34. The crystallographic orientations of the grains with and without the interface \# I. The $\left(\gamma+\gamma^{\prime}\right)$ coupled and $\left(\gamma+\gamma^{\prime}+\beta\right)$ eutectic structures showed the clear interface \# I, but the $\gamma^{\prime}$ planar and $\left(\gamma^{\prime}+\beta\right)$ eutectic structures did not show that 


\section{CONCLUSIONS}

1. Two distinct interfaces (\#I and \#II) occurs in the region of the solid-liquid growth front upon quenching of directionally solidified alloys of $\mathrm{Ni}$ plus $\mathrm{Al}$ having compositions near the $\mathrm{Ni}_{3} \mathrm{Al}$ compound. The interfaces occur with etching in a variety of etchants, with electropolishing, and with ion beam etching. Both interfaces are $\gamma^{\prime} / \gamma^{\prime}$ interfaces.

2. Experiments which varied the quench rate have shown that upon quencing two bands of $\gamma^{\prime}$ form upon the solid growth front, and interface \# II is located between these two $\gamma^{\prime}$ bands, the $\gamma^{\prime}$ lower band and the $\gamma_{\text {upper }}$ band. Interface \# I is the bottom of the $\gamma^{\prime}$ lowerband for the case where the growing solid is $\gamma^{\prime}$ phase, but for the case where the growing solid is $\gamma$ phase the bottom of the $\gamma^{\prime}$ lower quench band is a $\gamma^{\prime} / \gamma$ boundary.

3. SACP experiments in the SEM have have shown that there is no crystallographic orientation change across these boundaries. Standard diffraction experiments in the TEM as well as convergent beam diffraction experiments have confirmed that there is no orientation change across the interface and have found no fringe patterns to give evidence of any type of a disorder boundary at these interfaces.

4. Carful EDS experiments using low voltage excitation to minimize beam spreading in an SEM as well as thin foil TEM experiments have shown that interface \# II contains a small increase of Al while interface \# I contains a small depletion of Al. Models of the formation of these interfaces account for the observed composition changes as follows: Interface \# II is thought to form as a result of the nucleation of a thin $\beta$ phase layer which forms at the growth front of the $\gamma^{\prime}$ lower band that has grown upward from the solid-liquid interface with a planar or a cusped planar front upon quenching. A eutectic solid then nucleates on the $\beta$ film and 
developes a cellular eutectic sheet morphology which grows upward with clusters of parallel sheets of different orientations until they meet with the eutectics sheets which grow inward as clusters of parallel orientations from the quenched liquid above the advancing solid-liquid interface. Then, all the eutectic structures are transformed to the $\gamma^{\prime}$ phase on cooling to room temperature if the quench rate is not so fast. The $\beta$ film which forms at the top of the $\gamma^{\prime}$ lower band is thought to occur because of an $\mathrm{Al}$ build up in front of the advancing $\gamma^{\prime}$ lower band. This $\beta$ phase film is transformed to $\gamma^{\prime}$ on cooling but leaves an Al-rich boundary that causes the interface \# II to appear on etching. Interface \# I does not appear unless some $\gamma$ phase is growing with the $\gamma^{\prime}$ phase. It is postulated that the presence of this $\gamma$ phase nucleates a thin layer of $\gamma$ solid which grows laterally over the neighboring $\gamma^{\prime}$ phase followed by growth of $\gamma^{\prime}$ phase above it, and that this thin $\gamma$ layer transforms to $\gamma^{\prime}$ upon cooling except when the cooling rate is very fast. The thin layer of lateral growing $\gamma$ solid is the disordered FCC phase. It has alower Al content which results in the visibility of interface \# I after etching.

5. The occurrence of interface \# I is an interesting and very useful phenomenon because it outlines the position of the $\gamma$ growth front at the instant of the quench. Hence, it may be used to show the position of the growth front in coupled growth experiments involving the $\gamma$ and $\gamma^{\prime}$ phases.

6. It is found that the etching characteristics of $\mathrm{Ni} 3 \mathrm{Al}\left(\gamma^{\prime}\right)$ are very sensitive to small composition varitations as well as orientations. This result provides an important information to characterize the microstructure of $\mathrm{Ni}_{3} \mathrm{Al}$. 


\section{REFERENCES}

1. Goldstein, J.I.; Newbury, D.E.; Echlin, P.; Joy, D.C.; Fiori, C.; Lifshin, E. "Scanning Electron Microscopy and X-ray Microanalysis," Plenum Press, New York, NY, $1981,108$.

2. Janssen, M.M.P., Met. Trans., 1973, 4, 1623.

3. Jost, W., Diffusion in Solids, Liquids, Gases, Academic Press, New York, NY, 1952, 23. 
PAPER IV. CHARACTERISTICS OF EUTECTIC MICROSTRUCTURES NEAR $\mathrm{Ni}_{3} \mathrm{Al}$ COMPOSITIONS 


\section{INTRODUCTION}

The eutectic transformation is very common in metallurgy. A lot of study has been done and its theoretical model is well established. The eutectic structure has produced increased activity because of its good mechanical properties of aligned composites. The in-situ composite which includes intermetallic lamellae has been of special interest for high temperature services [1-4].

The nickel aluminide eutectic on the $\mathrm{Al}$ rich side $\left(\mathrm{Al}-\mathrm{Al}{ }_{3} \mathrm{Ni}\right)$ has been well studied [1, 58]. However, the eutectic near $\mathrm{Ni}_{3} \mathrm{Al}$ composition has never been studied and it was not well established whether the eutectic was composed of $\mathrm{Ni}_{3} \mathrm{Al}\left(\gamma^{\prime}\right)-\mathrm{NiAl}(\beta)$ or $\mathrm{Ni}_{3} \mathrm{Al}\left(\gamma^{\prime}\right)-\mathrm{Ni}(\gamma)$. The reason for the uncertainty is the interesting features of the phase diagram near $\mathrm{Ni}_{3} \mathrm{Al}$ compositions. The composition range of the $\gamma^{\prime}$ phase lies directly below the eutectic composition at high temperatures in the phase diagram. It causes the eutectic structure to change to the $\gamma^{\prime}$ single phase by solid state transformation on cooling and the eutectic could not be observed at room temperature. Another interesting feature is that the compositions and temperatures of eutectic and peritectic are very close and one of the eutectic phases is a peritectic phase. This feature might cause a meta-stable eutectic structure by reactions between the eutectic and a meta-stable phase or between the meta stable eutectic and a peritectic phase. Because the eutectic might transform to $\gamma^{\prime}$ single phase and react with the peritectic or metastable phase, directional solidification is a powerful technique to observe formation and transformation of eutectic at the solid liquid interface.

It was found that the $\gamma^{\prime}$ and $\beta$ phases form the eutectic transformation in MS thesis [9], but that study was not adeguate to explain some unusual eutectic structures that were found. Several questions arose here. The solid-liquid interface morphology of the eutectic was a cellular interface (not exact planar) and the eutectic spacing was unusually large. Also, the $\gamma^{\prime}$ phase in $\gamma^{\prime}+\beta$ eutectic included $\gamma$ precipitates in the center region of $\gamma^{\prime}$ at fast grow rates but did 
not include $\gamma$ precipitates at slow growth rates. This $\gamma^{\prime}$ phase including $\gamma$ precipitates $\left(\gamma+\gamma^{\prime}\right.$ mixed phase) could be originated from either the $\gamma$ phase or the $\gamma^{\prime}$ phase at the growth front because the precipitate phases could be forming in either primary phase during the quench procedure. If the mixed phase at high rates is the $\gamma^{\prime}$ phase before solid state transformation, it is strange not to find the mixed phase in the $\gamma$ phase of the eutectic at low rates. If the $\gamma^{\prime}+\gamma$ mixed phase was the $\gamma$ phase originally, then pure $\gamma$ phase must be present at the growth front and the growth front must be the ternary eutectic of $\gamma+\gamma^{\prime}+\beta$. A ternary eutectic in a binary has not previously been reported and it violates the equilibrium requirements of Gibbs phase rule. The possibility of the metastable $\gamma+\beta$ eutectic could be suggested if the $\left(\gamma+\gamma^{\prime}\right)$ mixed phase was all $\gamma$ phase initially and the $\gamma^{\prime}$ phase formed between $\gamma$ and $\beta$ during the quench procedure.

Any possibility could not be rejected because the solid state transformation is too fast to suppress the phase transformation during quench procedure in this system. Also, a meta-stable phase might be formed because of the closeness of the eutectic and peritectic temperature in this system.

It was interesting to find several different types of eutectics near the eutectic composition in this system. The various structures are shown in Figure 1 where a code has been assigned to them. In terms of numbers of phases, the eutectics of two phases $\left(\gamma^{\prime}+\beta\right)$ and three phases $\left\{\gamma^{\prime}+\beta+\left(\gamma+\gamma^{\prime}\right)\right.$ mixed $\}$ were found and termed as $2 \mathrm{P}$ and $3 \mathrm{P}$ respectively. In terms of morphologies in the cross section views, the eutectics of the lamellar, close packed rod, and cellular rod were found and termed as $L, R(c p), R(c)$ respectively. The $R(c)$ structure was unusual as the eutectic formed around a $y^{\prime}$ cell in cross section but the growth front was planar in longitudinal section. The eutectic structure was found at the eutectic composition as well as hypo and hyper eutectic compositions. Paper I showed that only the 3P-L eutectic was found as interdendritic structure at high solidification rate of $61 \mu \mathrm{m} / \mathrm{s}$ in hypo and hyper eutectic compositions. 


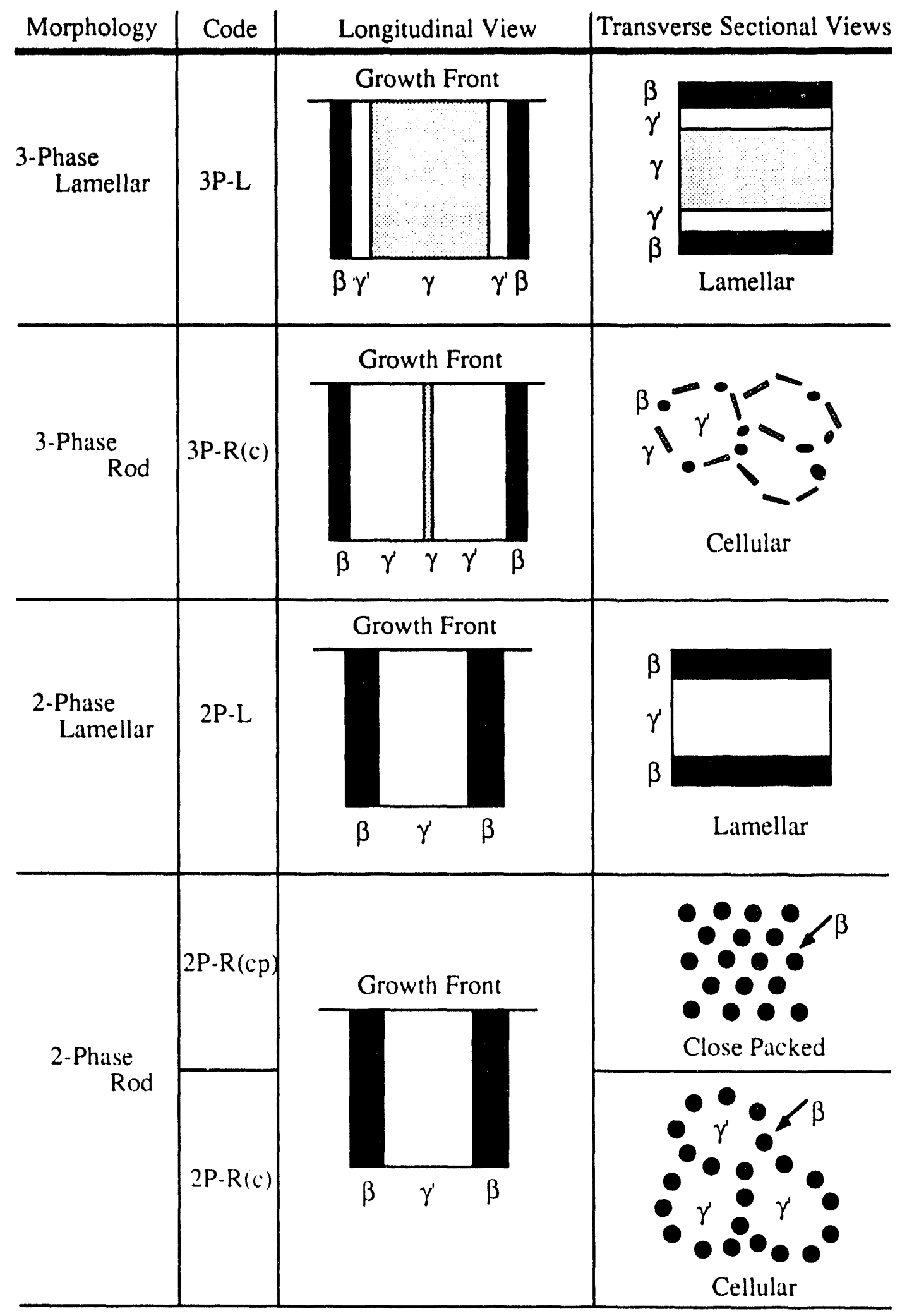

Figure 1. Schematic diagram of several kinds of eutectic morphologies 
However, several eutectic structures were found at low rates $(0.8-3.2 \mu \mathrm{m} / \mathrm{s})$. In hyper eutectic alloys (Al 25, 26, and 27), the $2 \mathrm{P}$ eutectics of the $\mathrm{R}(\mathrm{cp})$ and $\mathrm{L}$ types, which are classical eutectic structures, were found as a planar off eutectic structure or a interdendritic structure. In the eutectic alloy ( $\mathrm{A} \mid 24.5)$, the 3P eutectics of $\mathrm{L}$ and $\mathrm{R}(\mathrm{c})$ types were found as a planar interface. In hypo eutectic alloys(Al 23 and 24 alloys), a $\left(\gamma+\gamma^{\prime}\right)$ coupled structure was found at normal solidification fraction $(f s \approx 0.40)$ as a interdendritic or as a planar interface. The $\gamma+\gamma^{\prime}$ coupled structure was a different structure to the eutectic structure. The longitudinal section looked like the eutectic structure but the cross section was $\gamma^{\prime}$ cellular type. The $\gamma+\gamma^{\prime}$ couple structure appeared as the coupled growth of peritectic phases. At high G/V (gradient/rate) conditions of $0.8 \mu \mathrm{m} / \mathrm{s}$ the $\gamma+\gamma^{\prime}$ band structure was also found instead of the $\gamma+\gamma^{\prime}$ coupled structure. At higher solidification fractions ( $\mathrm{fs}>0.55$ in $\mathrm{Al} 23$ alloy and $\mathrm{fs}>0.44$ in $\mathrm{Al} 24$ alloy) the $\gamma+\gamma^{\prime}$ coupled peritectic structure (or the band structure at high $\mathrm{G} / \mathrm{V}$ ) changed to the $y^{\prime}$ planar and then the eutectic structures.

It was an important result to find the coupled growth in the solidification of a peritectic system. It is widely accepted in the literature that although coupled growth of eutectic alloys at an isothermal growth front is a common growth mode, the coupled growth of the two phases of a peritectic alloy at an isothermal growth front does not occur [10-12]. Directional solidification in peritectic systems have consistently found a band structure, where first one phase of the peritectic reaction occurs followed by the second phase. The possibility of the coupled growth of peritectic has been of special interest, but only initial experiments have been completed. Directional solidification experiments on the $\gamma^{\prime} / \gamma$ peritectic in hypo eutectic alloys have shown that a transition between the two mode is controlled by the ratio of $G / V$, the ratio of temperature gradient to growth velocity. However, further experiments are required to understand the mechanisms and conditions for the coupled growth and band structures in hypo eutectic alloys. This study is currently underway as another subject, so only the eutectic study is presented in this paper. 
In order to understand the mechanism of unusual eutectic structures formed near $\mathrm{Ni}_{3} \mathrm{Al}$ compositions, directional solidification experiments were done at various velocities with compositions near $\mathrm{Ni}_{3} \mathrm{Al}$. The possibility of the metastable eutectic or three phase eutectic, which might be formed in this system, was investigated. The effect of impurities on the eutectic was also investigated. 


\section{EXPERIMENTS AND RESULTS}

Directional solidification experiments were accomplished by controlling growth rates and compositions, as described in Paper I. Several different temperature gradients were also obtained here, as described in the Appendix.

The alloys were prepared near $\mathrm{Ni}_{3} \mathrm{Al}$ compositions; $23,24,24.5,25,25.5,26$, and 27 at\% $\mathrm{Al}$ (these alloys will be designated $\mathrm{Al} 23, \mathrm{Al} 24, \mathrm{Al} 24.5$, and so on), where the alloy preparation procedure was described in Part I. Experiments were done at various growth rates near the eutectic compositions. Table 1 shows all the experiments studied to find eutectic structures in the eutectic, hypo, and hyper eutectic compositions. Two different batches of $\mathrm{Al}$ 23.5 and 24.5 alloys, made using different sources of $\mathrm{Ni}$, were prepared and these are called Al 23.5(I and II) and 24.5(I and II) in Table 1. The 23.4(I) alloy was shown to have been contaminated with impurities and it will be discussed later. A pure eutectic structure with no dendrites was only found in the Al 24.5 (I) and (II) alloys at the highest rate studied, $61 \mu \mathrm{m} / \mathrm{s}$. Therefore, the compositions of 24.5 at $\%$ Al will be considered to be the eutectic composition. The eutectic structure was found as an interdendritic phase in both hypo and hyper eutectic composition ranges at high growth rates. The off-eutectic plane front structure was found at low rates in hyper eutectic compositions of $\mathrm{Al} 25$ and 26 alloys. The plane front eutectic structure was also obtained in hypo eutectic alloys at low rates at the end position of directionally solidified rods after the primary planar $(\gamma)$ and secondary planar $\left(\gamma^{\prime}\right)$ structures had formed. The eutectic structures were formed with the $\gamma^{\prime}$ phase between the dendrites as a separated $\gamma^{\prime}$ cell (named $\gamma^{\prime}$ cell in Table 1) or the $\gamma^{\prime}$ phase around the dendrites (expressed as dendrite $+\gamma^{\prime}$ in Table 1) at relatively high rates (above $3.2 \mu \mathrm{m} / \mathrm{s}$ ), where the growth front was almost the same or the r' phase lead a little bit.

Several kinds of eutectic morphologies were found here; two phase $\left(\gamma^{\prime}+\beta\right)$ or three phase $\left(\gamma^{\prime}+\beta+\left(\gamma+\gamma^{\prime}\right)\right.$ mixed) eutectics of lamellar, rod, and cellular types. In the cellular type, 
Table 1 All the eutectic structures near the eutectic composition

\begin{tabular}{|c|c|c|c|c|c|c|}
\hline Alloy & $\mathrm{V}(\mu \mathrm{m} / \mathrm{s})$ & Exp.\# & $\begin{array}{l}\mathrm{G} / \mathrm{V}\left(10^{5}\right. \\
\left.\mathrm{Cs} / \mathrm{cm}^{2}\right)\end{array}$ & fs & Interface & Eutectic Structure \\
\hline Al 27 & $\begin{array}{l}0.8 \\
3.2 \\
61 \\
\end{array}$ & $\begin{array}{l}25 \\
24 \\
18 \\
\end{array}$ & $\begin{array}{l}3.14 \\
0.78 \\
0.04 \\
\end{array}$ & $\begin{array}{l}0.45 \\
0.60 \\
0.51 \\
\end{array}$ & $\begin{array}{c}\beta \text {-Planar } \\
\beta \text {-dend }+\gamma \\
\beta \text {-dend., } \gamma \text { cell } \\
\end{array}$ & $\begin{array}{c}2 \mathrm{P} \cdot \mathrm{L}+2 \mathrm{P} \cdot \mathrm{R}(\mathrm{cp}) \\
3 \mathrm{P} \cdot \mathrm{L}\end{array}$ \\
\hline Al 26 & $\begin{array}{l}0.8 \\
3.2 \\
61\end{array}$ & $\begin{array}{l}38 \\
32 \\
29\end{array}$ & $\begin{array}{l}3.14 \\
0.78 \\
0.04\end{array}$ & $\begin{array}{l}0.32 \\
0.75 \\
0.34\end{array}$ & $\begin{array}{l}\text { Eut. Planar } \\
\beta \text {-dend. }+\gamma \\
\beta \text {-dend. } \gamma \text { cell }\end{array}$ & $\begin{array}{c}2 \mathrm{P} \cdot \mathrm{L} \\
2 \mathrm{P} \cdot \mathrm{L}+2 \mathrm{P} \cdot \mathrm{R}(\mathrm{cp}) \\
3 \mathrm{P} \cdot \mathrm{L}\end{array}$ \\
\hline Al 25 & $\begin{array}{l}0.8 \\
3.2 \\
5.0 \\
6.3 \\
10 \\
25 \\
25 \\
61\end{array}$ & $\begin{array}{c}4 \\
19 \\
54 \\
3 \\
53^{* *} \\
52^{* *} \\
23 \\
8\end{array}$ & $\begin{array}{l}3.14 \\
0.78 \\
0.50 \\
0.39 \\
0.25 \\
0.09 \\
0.09 \\
0.04\end{array}$ & $\begin{array}{l}0.30 \\
0.44 \\
0.49 \\
0.74 \\
0.56 \\
0.58 \\
0.40 \\
0.57\end{array}$ & $\begin{array}{c}\text { Eut. Planar } \\
" " \\
" " \\
\beta \text {-dend. }+\gamma \\
\beta \text {-dend., } \gamma \text { cell } \\
\beta \text {-dend., } \gamma \text { cell } \\
\beta \text {-dend., } \gamma \text { cell }\end{array}$ & $\begin{array}{c}2 \mathrm{P}-\mathrm{R}(\mathrm{cp}) \\
2 \mathrm{P}-\mathrm{R}(\mathrm{cp})+2 \mathrm{P}-\mathrm{L} \\
2 \mathrm{P}-\mathrm{R}(\mathrm{cp})+2 \mathrm{P}-\mathrm{L}+2 \mathrm{P}-\mathrm{R}(\mathrm{c}) \\
2 \mathrm{P}-\mathrm{L}+2 \mathrm{P}-\mathrm{R}(\mathrm{cp}) \\
2 \mathrm{P}-\mathrm{R}(\mathrm{c}) \\
3 \mathrm{P}-\mathrm{L}+2 \mathrm{P}-\mathrm{R}(\mathrm{c}) \\
3 \mathrm{P}-\mathrm{L}+2 \mathrm{P}-\mathrm{R}(\mathrm{c}) \\
3 \mathrm{P}-\mathrm{L}\end{array}$ \\
\hline Al $24.5(1)^{*}$ & $\begin{array}{l}0.8 \\
0.8 \\
3.2 \\
3.2 \\
5.0 \\
5.0 \\
25 \\
61 \\
\end{array}$ & $\begin{array}{c}121 \\
119 \\
59 \\
98 \\
61 \\
62 \\
58 \\
129 \\
\end{array}$ & $\begin{array}{l}5.18 \\
5.18 \\
0.78 \\
1.28 \\
0.50 \\
0.50 \\
0.09 \\
0.05 \\
\end{array}$ & $\begin{array}{l}0.60 \\
0.47 \\
0.42 \\
0.42 \\
0.42 \\
0.44 \\
0.40 \\
0.65 \\
\end{array}$ & $\begin{array}{c}\text { Eut. Planar } \\
\gamma \text { Planar, Eut. Planar } \\
\text { Eut. Planar } \\
" " \\
" ~ \\
\gamma^{\prime} \text { cell, Eut. Planar } \\
\text { Eut. Planar }\end{array}$ & $\begin{array}{c}2 \mathrm{P}-\mathrm{R}(\mathrm{c})+3 \mathrm{P}-\mathrm{R}(\mathrm{c}) \\
3 \mathrm{P}-\mathrm{L}+3 \mathrm{P}-\mathrm{R}(\mathrm{c}) \\
2 \mathrm{P}-\mathrm{R}(\mathrm{c})+3 \mathrm{P}-\mathrm{R}(\mathrm{c}) \\
" \\
" \\
3 \mathrm{P}-\mathrm{L} \\
3 \mathrm{P}-\mathrm{L} \\
\end{array}$ \\
\hline $\mathrm{Al} 24.5$ (II) & $\begin{array}{l}0.8 \\
0.8 \\
0.8 \\
3.2 \\
5.0 \\
10 \\
61 \\
\end{array}$ & $\begin{array}{l}115 \\
117 \\
126 \\
118 \\
110 \\
116 \\
128 \\
\end{array}$ & $\begin{array}{l}5.18 \\
5.18 \\
5.18 \\
1.28 \\
0.81 \\
0.39 \\
0.05 \\
\end{array}$ & $\begin{array}{l}0.40 \\
0.40 \\
0.21 \\
0.47 \\
0.34 \\
0.50 \\
0.65 \\
\end{array}$ & $\begin{array}{l}\text { Eut. Planar } \\
\text { " } \\
\text { " } \\
" \\
" \\
"\end{array}$ & $\begin{array}{c}3 \mathrm{P}-\mathrm{L} \\
" \\
" \\
3 \mathrm{P}-\mathrm{L}+3 \mathrm{P}-\mathrm{R}(\mathrm{c}) \\
3 \mathrm{P}-\mathrm{L}+3 \mathrm{P}-\mathrm{R}(\mathrm{c})+2 \mathrm{P}-\mathrm{R}(\mathrm{c}) \\
" 1 \\
3 \mathrm{P}-\mathrm{L}\end{array}$ \\
\hline $\mathrm{Al} 24$ & $\begin{array}{l}0.8 \\
3.2 \\
61 \\
\end{array}$ & $\begin{array}{l}125 \\
105 \\
25 \\
\end{array}$ & $\begin{array}{l}5.18 \\
1.28 \\
0.04 \\
\end{array}$ & $\begin{array}{l}0.91 \\
0.90 \\
0.64 \\
\end{array}$ & $\gamma$ dend., $\gamma$ cell & $\begin{array}{c}2 \mathrm{P}-\mathrm{R}(\mathrm{c}) \\
2 \mathrm{P}-\mathrm{L}+2 \mathrm{P}-\mathrm{R}(\mathrm{c}) \\
3 \mathrm{P}-\mathrm{L}\end{array}$ \\
\hline $\begin{array}{l}\text { Al } 23.5(\mathrm{I})^{*} \\
\text { Conta- } \\
\text { minated }\end{array}$ & $\begin{array}{l}0.8 \\
0.8 \\
2.0 \\
2.0 \\
3.2 \\
3.2 \\
3.2 \\
\end{array}$ & $\begin{array}{c}99 \\
107 \\
100 \\
103 \\
95 \\
92 \\
102 \\
\end{array}$ & $\begin{array}{l}5.18 \\
5.18 \\
2.06 \\
2.06 \\
1.28 \\
1.28 \\
1.28 \\
\end{array}$ & $\begin{array}{l}0.49 \\
0.83 \\
0.41 \\
0.91 \\
0.24 \\
0.39 \\
0.68\end{array}$ & $\begin{array}{c}\text { Eut. Planar } \\
" \\
" \\
" \\
\gamma \text { dend. } \\
\text { Eut. Planar } \\
\text { " } \\
\end{array}$ & $\begin{array}{c}\text { 3P-L } \\
" \\
" \\
" \\
" \\
" \\
"\end{array}$ \\
\hline $\mathrm{Al} 23.5(\mathrm{II})$ & $\begin{array}{l}0.8 \\
3.2 \\
\end{array}$ & $\begin{array}{l}120 \\
111 \\
\end{array}$ & $\begin{array}{l}5.18 \\
1.28 \\
\end{array}$ & $\begin{array}{l}0.74 \\
0.81\end{array}$ & $\begin{array}{l}\text { Eut. Planar } \\
\text { " }\end{array}$ & $\begin{array}{l}2 \mathrm{P}-\mathrm{R}(\mathrm{c})+3 \mathrm{P}-\mathrm{R}(\mathrm{c}) \\
2 \mathrm{P}-\mathrm{R}(\mathrm{c})+3 \mathrm{P}-\mathrm{R}(\mathrm{c})\end{array}$ \\
\hline Al 23 & $\begin{array}{l}0.8 \\
0.8 \\
1.1 \\
61\end{array}$ & $\begin{array}{l}106 \\
97 \\
85 \\
10 \\
\end{array}$ & $\begin{array}{l}5.18 \\
3.55 \\
3.76 \\
0.04\end{array}$ & $\begin{array}{l}0.87 \\
0.75 \\
0.98 \\
0.62\end{array}$ & $\begin{array}{l}\text { Eut. Planar } \\
", \\
\gamma \text { dend., } \gamma \text { cell }\end{array}$ & $\begin{array}{c}2 \mathrm{P}-\mathrm{L}+2 \mathrm{P}-\mathrm{R}(\mathrm{cp}) \\
2 \mathrm{P} \cdot \mathrm{L}+3 \mathrm{P}-\mathrm{R}(\mathrm{c}) \\
2 \mathrm{P}-\mathrm{R}(\mathrm{c}) \\
3 \mathrm{P}-\mathrm{L}\end{array}$ \\
\hline
\end{tabular}

INCO Ni was used for alloy preparation, but AO Nı was used for the * marked alloys of $23.5(\mathrm{I})$ and $24.5(\mathrm{I})$. The $23.5(\mathrm{l})$ alloy was contaminated by $\mathrm{Fe}, \mathrm{Cr}, \mathrm{Mn}, \mathrm{Si}$, and $\mathrm{Nb}$.

** were quenched to the Ga-In and the others were quenched to water. 
the two or three phase eutectics form around a $\gamma^{\prime}$ cell. These eutectic morphologies are classified in Figure 1 and the code shown in this figure has been used in Table 1.

Several unusual eutectic characteristics were found when these eutectic structures were compared with conventional eutectic structure. The solid-liquid interface morphology was planar but the quenched interfaces had a cellular appearance (see Figures 2(e), 2(g), 3). As will be shown later, the spacing of the $\gamma^{\prime}+\beta$ eutectic phase is larger than that of other eutectic systems by at least 10 times. The cellular type of rod eutectic was a new morphology of eutectic structure. It was also interesting to find a three phase eutectic $\left(\gamma^{\prime}+\beta+\left(\gamma^{\prime} \gamma^{\prime}\right)\right.$ mixed) in a binary system.

Classical Eutectic Structures and Solid-liquid Interface Morphology As $G / V$ increased to $(G / V)_{\mathrm{cr}}$, the $\beta$ dendrites shortened and disappeared in hyper eutectic alloys, as seen in Table 1. The classical morphologies of rod and lamellar types (2P-L and $2 \mathrm{P}-\mathrm{R}(\mathrm{cp})$ ) were found as planar interfaces at low rates in the hyper eutectic alloys of $\mathrm{Al} 25$ and 26, as shown in Figures 2 and 3. These eutectics must be off-eutectic plane front morphologies which form without $\beta$ dendrites. The $1(0) \%$ lamellar eutectic (2P-L) was found in Al 26 alloy and the lamellar+rod mixed structures were found in Al 25 alloy. The composition dependence of rod/lamellar morphologies is roughly as expected because the lamellar type eutectic is preferred at high volume fraction of the $\beta$ phase of AI 26 alloy, see phase diagram of Figure 9.

Even though the classical morphologies of the rod and lamellar eutectics were shown in the transverse section, the longitudinal morphology shows that the $\gamma^{\prime}$ phase leads the $\beta$ phase with the classical cellular shape, as shown in Figure 3. However, the cells did not disappear as the solidification rate was decreased by factor of $10\left(\right.$ from $6.3 \mu \mathrm{m} / \mathrm{s}$ to $(0.8 \mu \mathrm{m} / \mathrm{s})$, and the $\gamma^{\prime}$ cell with the $\beta$ phase at $6.3 \mu \mathrm{m} / \mathrm{s}$ changed to the $\beta$ dendrite at $10 \mu \mathrm{m} / \mathrm{s}$ in $\mathrm{Al} 25$ alloy, where if it were $\gamma^{\prime}$ cellular interface the $\gamma^{\prime}$ dendrite would form instead of the $\beta$ dendrite. 
a)

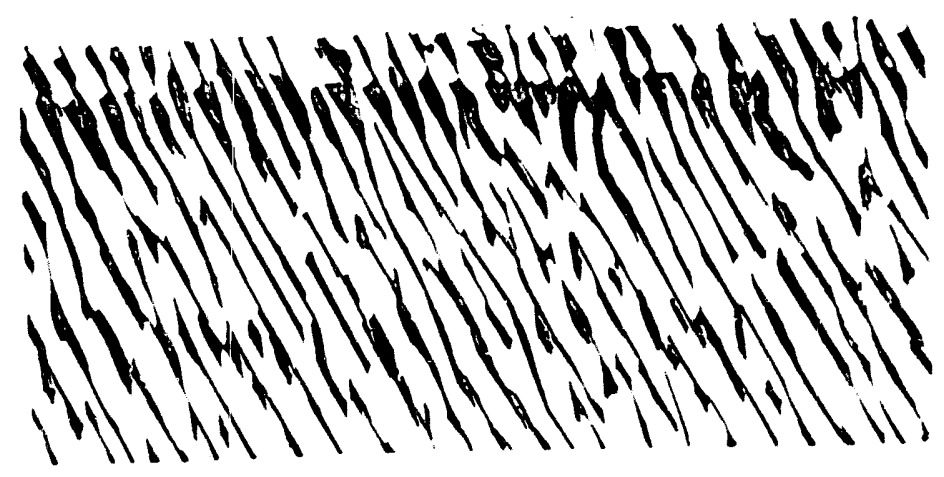

b)

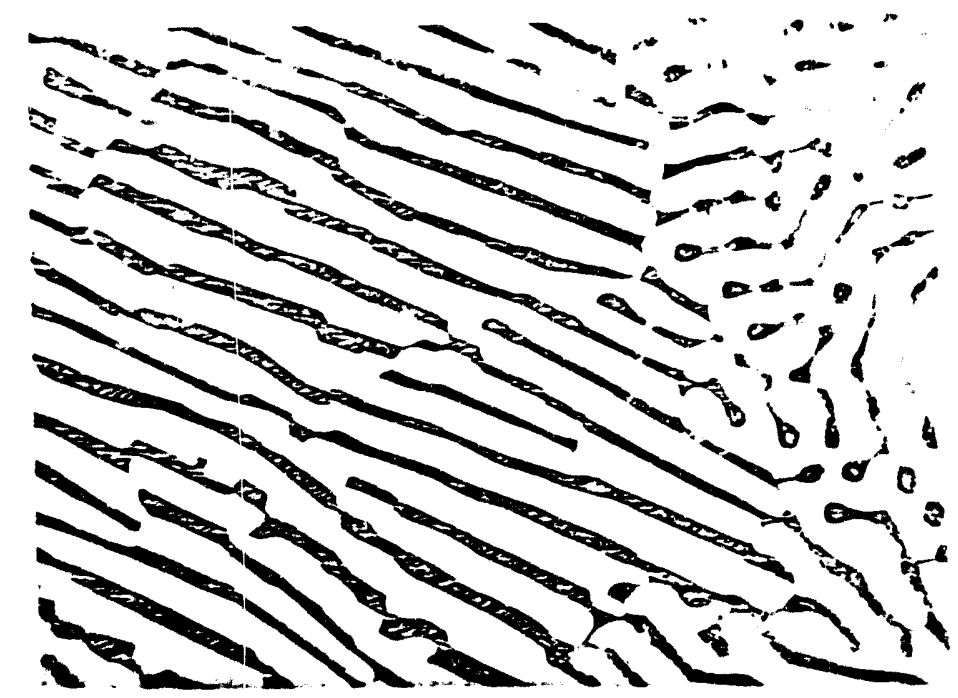

Figure 2. The eutectic morphologies of rod and lamellar types in the off-eutectic compositions of $\mathrm{Al} 25$ and 26 alloys. a) Longitudinal and b) transverse views in Exp.\# 38 (Al 26$0.8 \mu \mathrm{m} / \mathrm{s}), 50 \mathrm{x}$, 

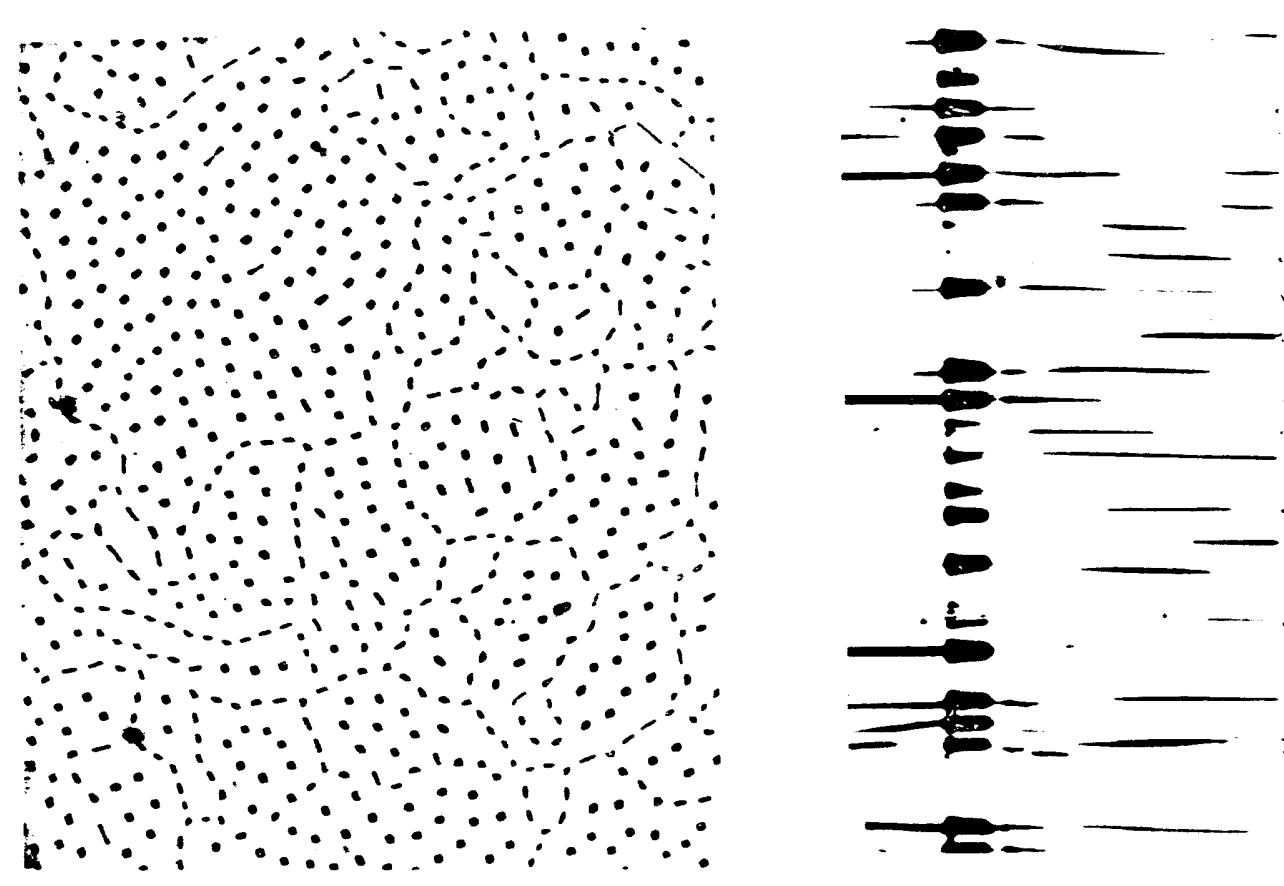

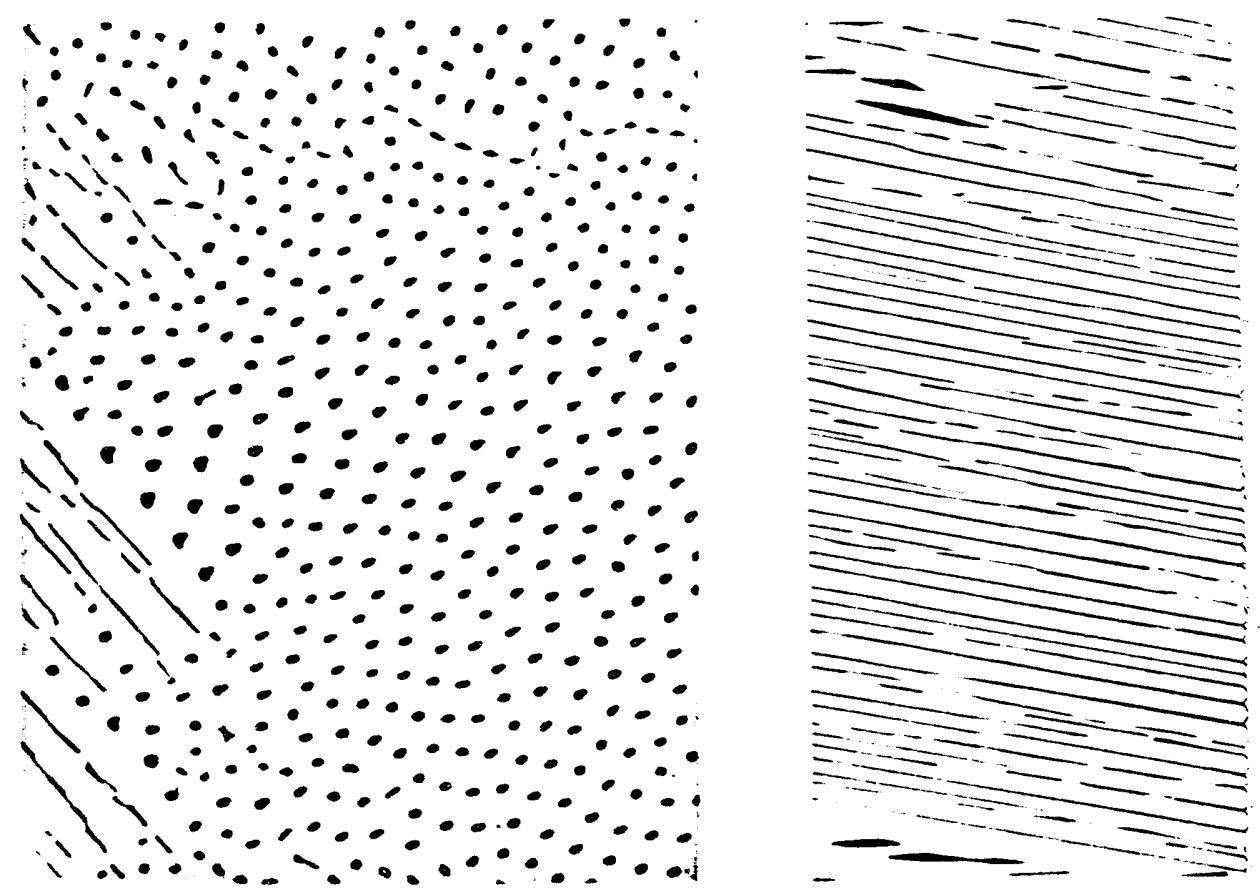
g)

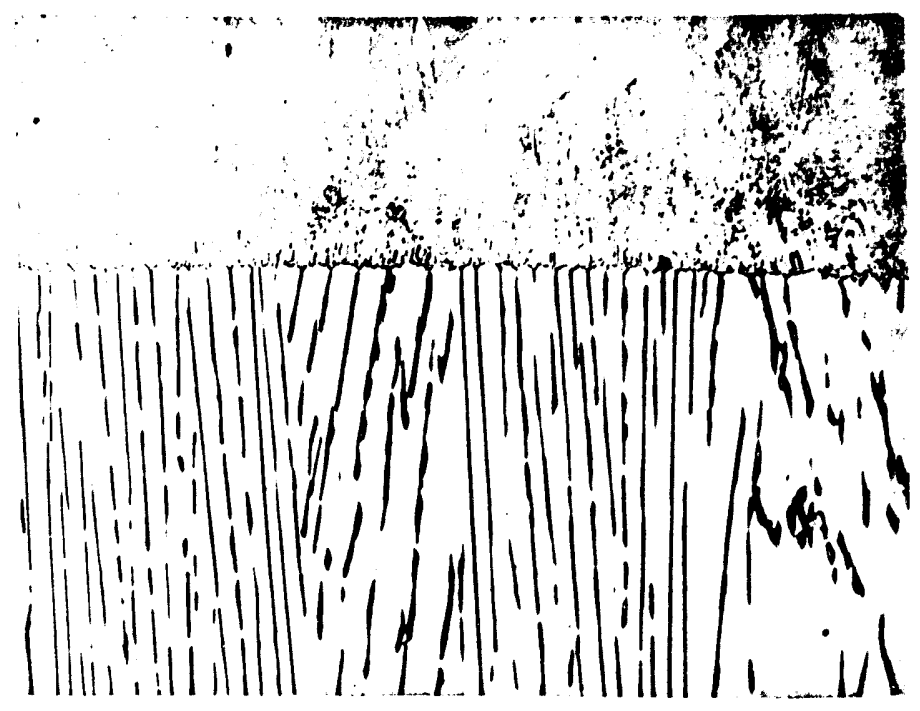

h)

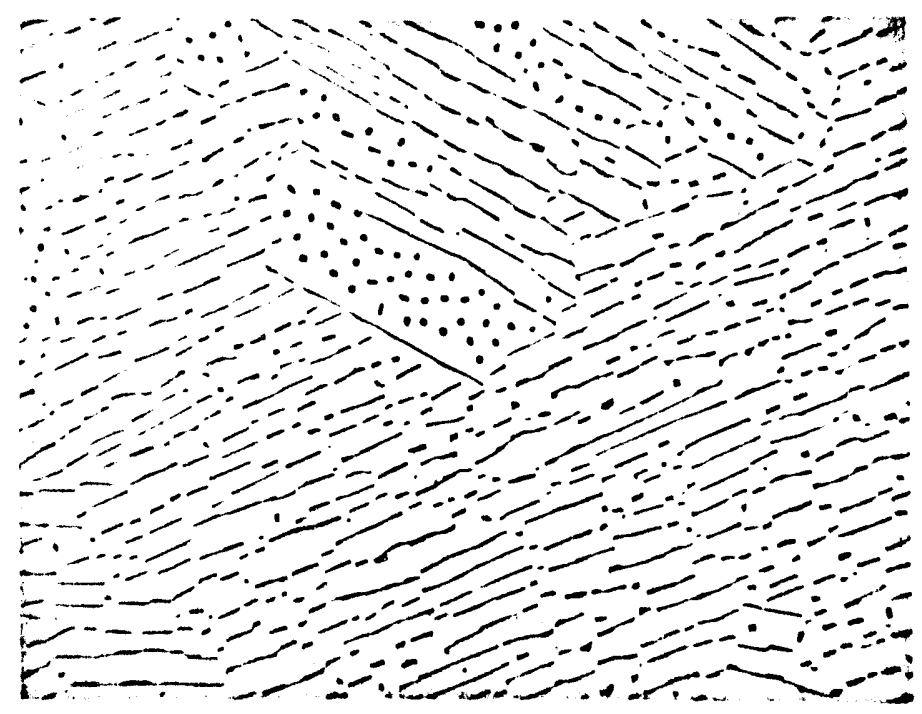

Figure 2. (continued) g) longitudinal and h) transeverse views in Exp.\# 3 (Al 25-6.3 $\mu \mathrm{m} / \mathrm{s}$ ), $50 \mathrm{x}$ 

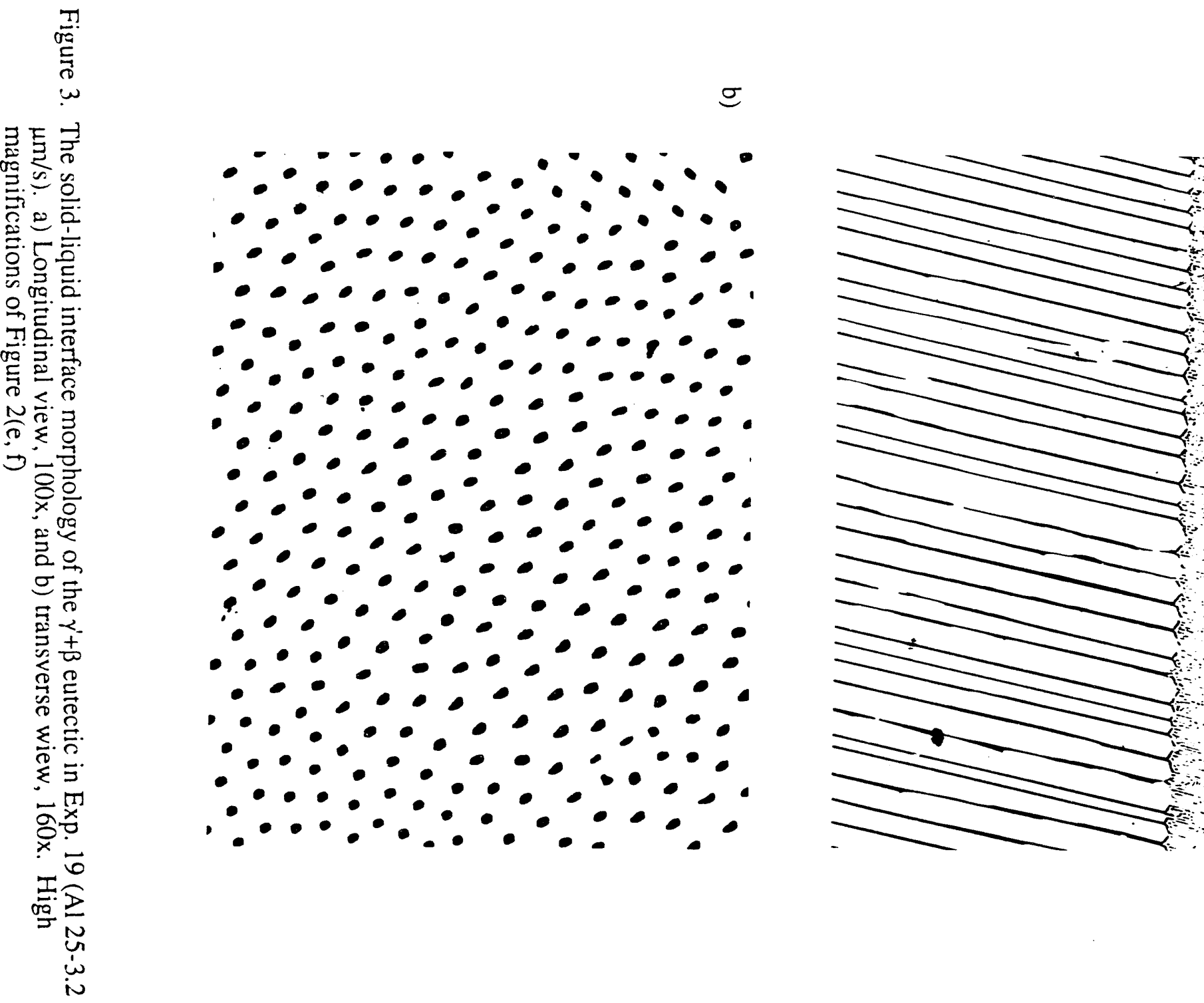
After studying the mystery interface \# I in the three phase eutectic (3P-R(c)) in Paper III, it could be concluded that the original solid-liquid interface of the eutectic was planar. A clear mystery interface \# I was not found at the $\gamma^{\prime}$ phase of the eutectic in the two phase eutectic growth front of $\gamma^{\prime}+\beta$. But, it always occurred at the three phase eutectic of $\left(\beta+\gamma^{\prime}+\left(\gamma+\gamma^{\prime}\right)\right.$ mixed phase), as shown in Figure 4(c) for a structure termed 3P-R(c). It was concluded in Part III that the $\gamma$ phase at the solid-liquid interface helps to show the original quenched interface by its fast lateral growth on quenching.

In conclusion, this morphology at the solid-liquid interface is thought to be a classical eutectic shape with a planar interface. The cellular appearance of the quenched interfaces results from $\gamma^{\prime}$ caps that form during quenching.

\section{Three Phase Eutectic}

While the conventional eutectic type of $\gamma^{\prime}+\beta$ eutectics was found at low rates with hyper eutectic compositions (Al 25 to 27 alloys), three phase eutectics of lamellar type (3P-L), Figures 4(a) and 4(b), or cellular type (3P-R(c)), Figures 4(c) and 4(d), were found at the eutectic composition alloy (AI 24.5 alloy). Always, the 3P-L eutectic was formed in AI 23.5 (I) alloy at either high rates and low rates as a planar or interdendritic structure. The 3P-L eutectic was also found as interdendritic structure at high solidification rates (above $25 \mu \mathrm{m} / \mathrm{s}$ ) of all alloy compositions. In hypo eutectic alloys (Al 23,23.5(II), 24 alloys), all kinds of eutectics, 3P-L, 3P-R(c), 2P-L, 2P-R(cp), and 2P-R(c), were found at low rates in the final solidification fraction after the growth of the $\gamma^{\prime}$ planar interface.

It was very strange to find three phase eutectic in binary and it has not been reported. Figure 4 shows two types of three phase eutectics, cellular and lamellar types. 


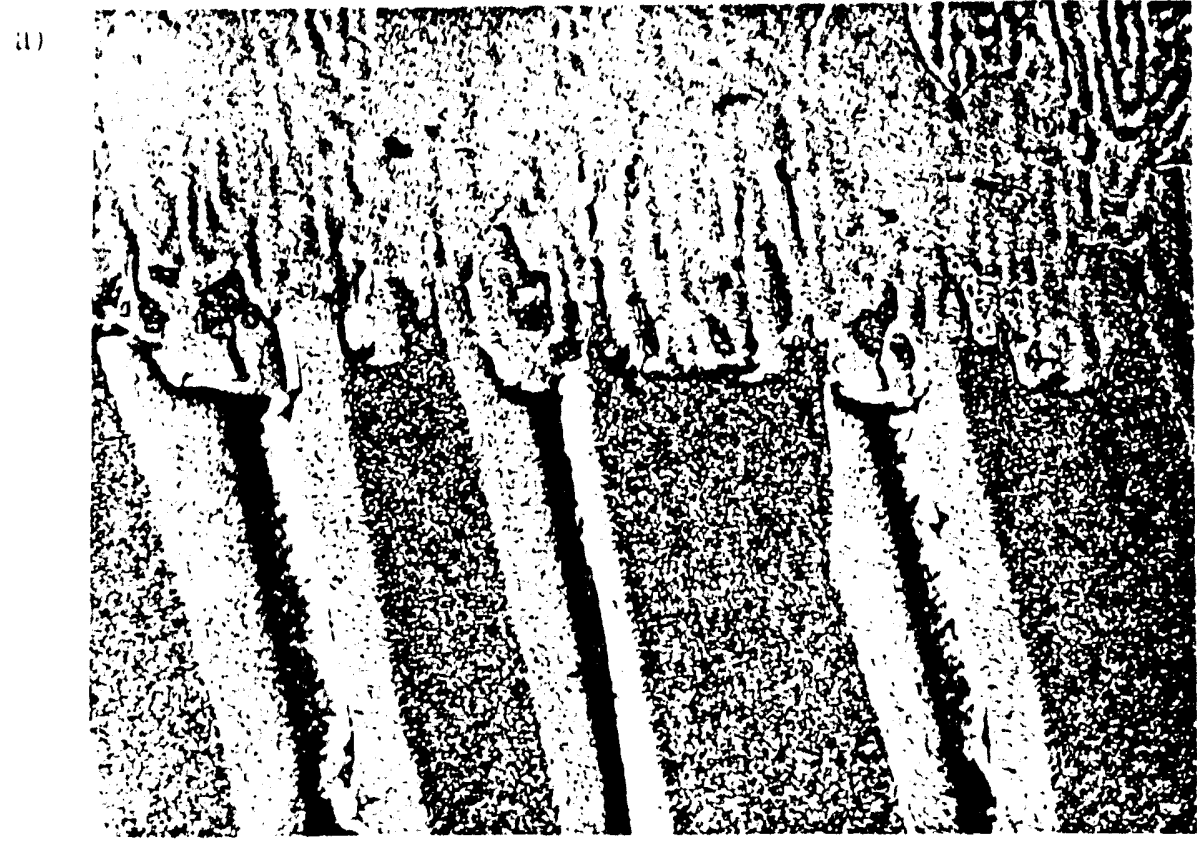

b)

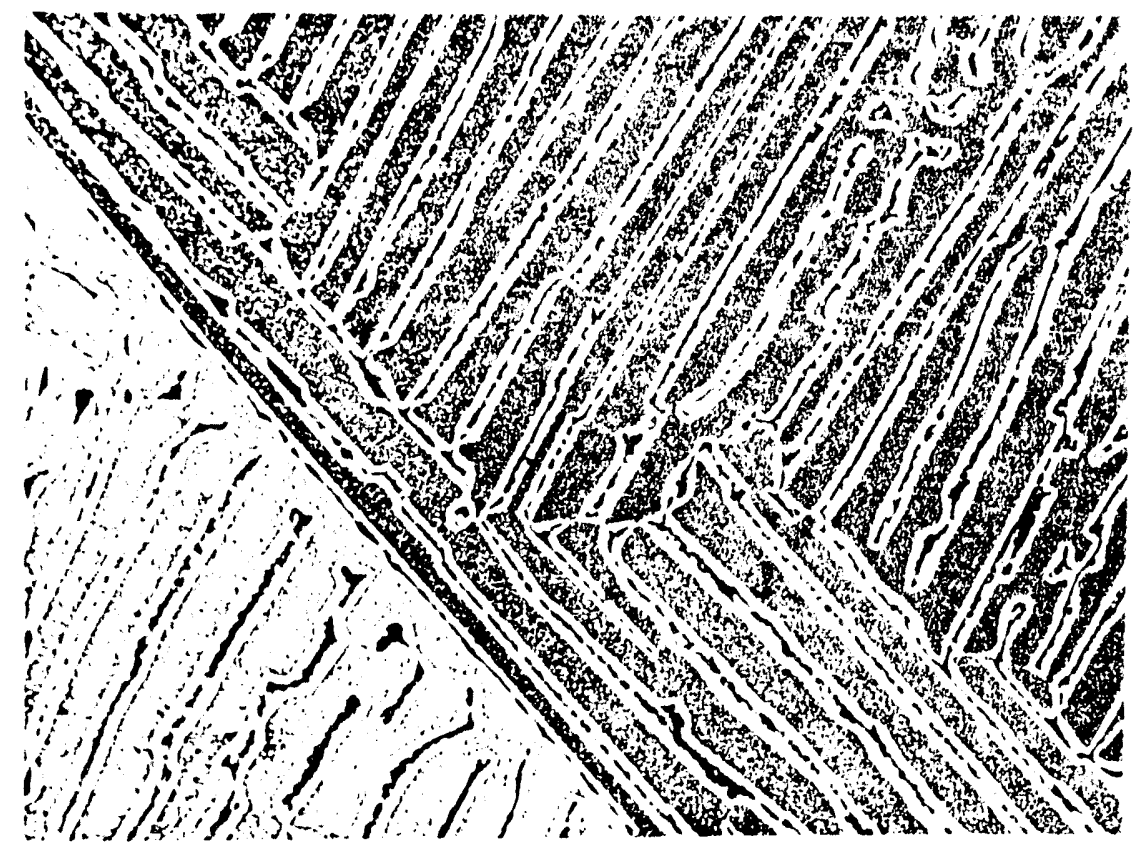

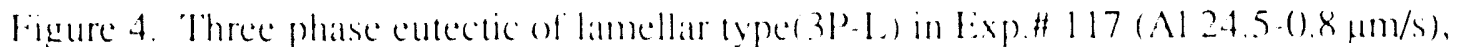

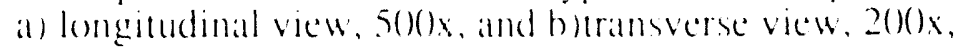


(c)

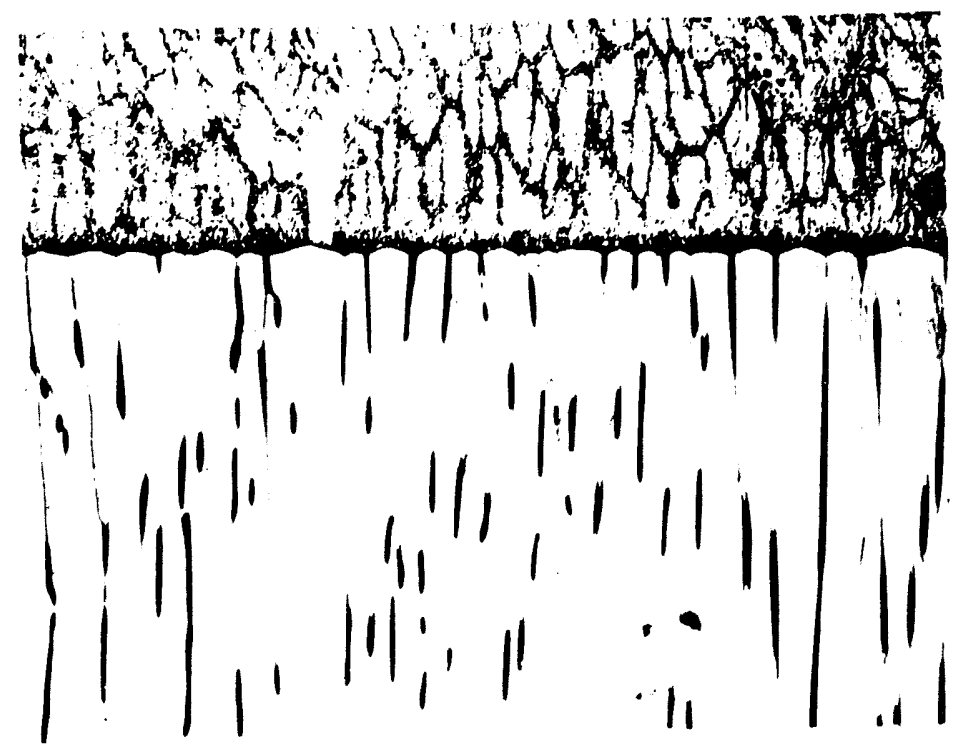

d)

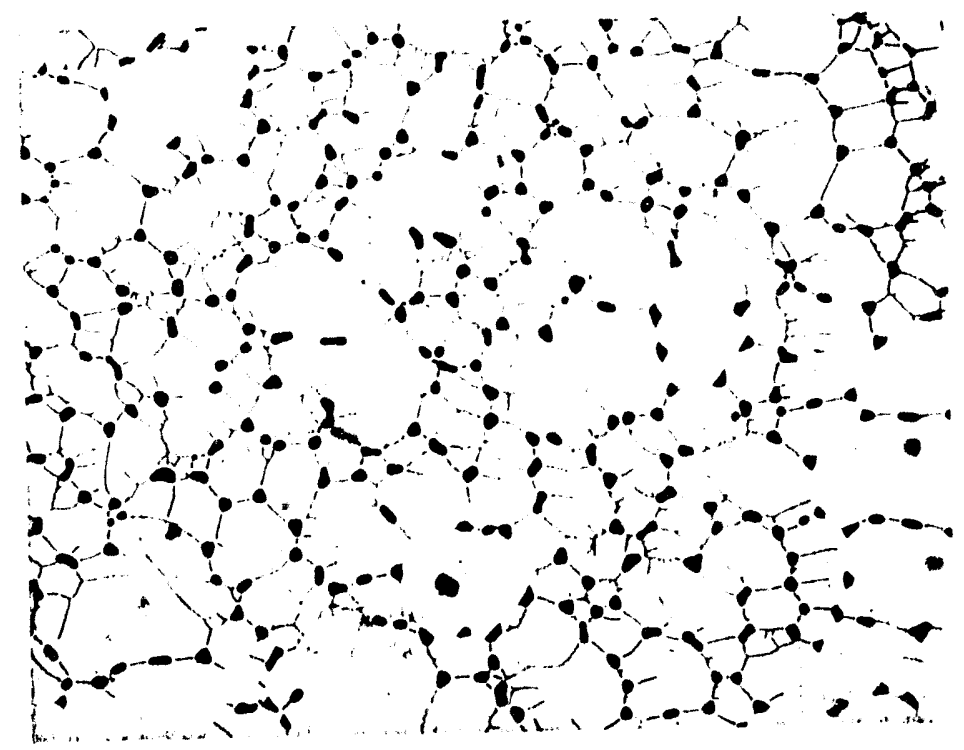

Figure 4. (continued) and three phase eutectic of cellular type(3P-R(c)) in Exp.\# 61(AI 24.5-5 $\mu \mathrm{m} / \mathrm{s}), \mathrm{c}$ ) longitudinal view, 5() $\mathrm{x}$, and transverse view, 10() $\mathrm{x}$ 


\section{Characterization of three phase eutectic}

The three phase eutectic samples were studied in the SEM and compositions were analyzed using EDS. The phase identification was evaluated from the etching characteristics of the three phases. While the $\beta$ phase was etched very easily, the $\gamma$ ' phase was attacked only a little and the $\gamma$ phase was not etched out in Marbles solution [9]. The tilted SEM micrograph in Figure 5 illustrates the etching characteristics of the three phases. It could be expected that the modeled phase labeled 1 is the $\gamma$ phase with $\gamma^{\prime}$ precipitates, the uniform phase labeled 2 is the $\gamma^{\prime}$ phase, and the deep etched phase is the $\beta$ phase. It could be also deduced that the major phase of the eutectic must be the $\gamma$ phase because the microstructure of the $\gamma$ dendrite tip of Figure 6 shows the same modeled structure as the major phase of the eutectic. The compositions of three regions were analyzed by EDS. Figure 7(a) shows the burn marks caused when the $x$-ray hit the surface, where low voltage $(5 \mathrm{kV})$ was used to reduce the diameter of sample volume producing $x$-rays. The expected maximum range was $0.3 \mu \mathrm{m}$ at 5 $k V$, as discussed in Paper III. The composition change between the $\beta$ phases shows that there is $\gamma^{\prime}$ region (about $24.5 \mathrm{at} \% \mathrm{Al}$ ) and $\gamma+\gamma^{\prime}$ mixed region (about 21.5 at\% $\mathrm{Al}$ ), as shown in Figure 7(b). The $\gamma+\gamma^{\prime}$ mixed phase must have been the $\gamma$ phase before the $\gamma^{\prime}$ phases precipitated on cooling. The $\beta$ phase region also included $\gamma^{\prime}$ precipitates which resulted from the quench procedure. The $\gamma^{\prime}$ precipitates in the $\beta$ phase region were so coarse that the average composition of the $\beta$ phase before solid state transformation could not be obtained. However, the $\gamma^{\prime}$ precipitates in the $\gamma$ phase region were fine enough to get the average composition of the $\gamma$ phase.

Three phase eutectic of cellular type (3P-R(c)) was also observed and analyzed in the SEM. Figure 8 shows that three phases form at the same growth front in the cellular type. The average composition of the $\gamma^{\prime}$ phase was $\mathrm{Al} 24 .()$ at.\% and the $\gamma$ phase (included $\gamma^{\prime}$ precipitates) was Al 21.1 at. \%. 


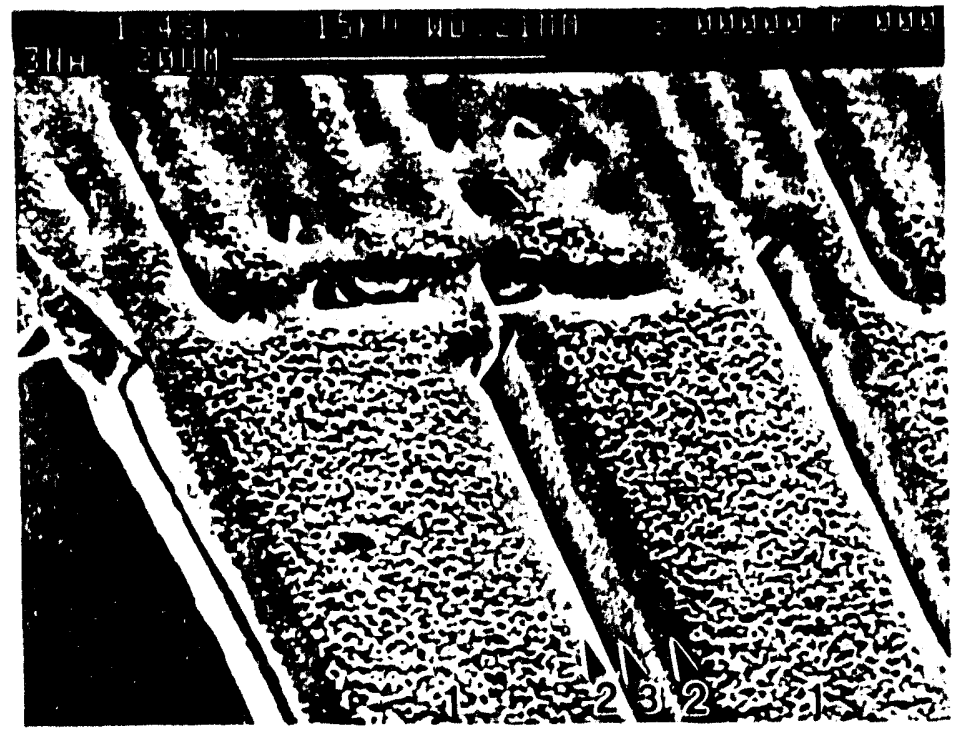

Figure 5. The tilted SEM micrograph in three phase lamellar type eutectic(3P-L) in Exp. 95 (Al $23.5(\mathrm{I})-3.2 \mu \mathrm{m} / \mathrm{s})$ 
a)

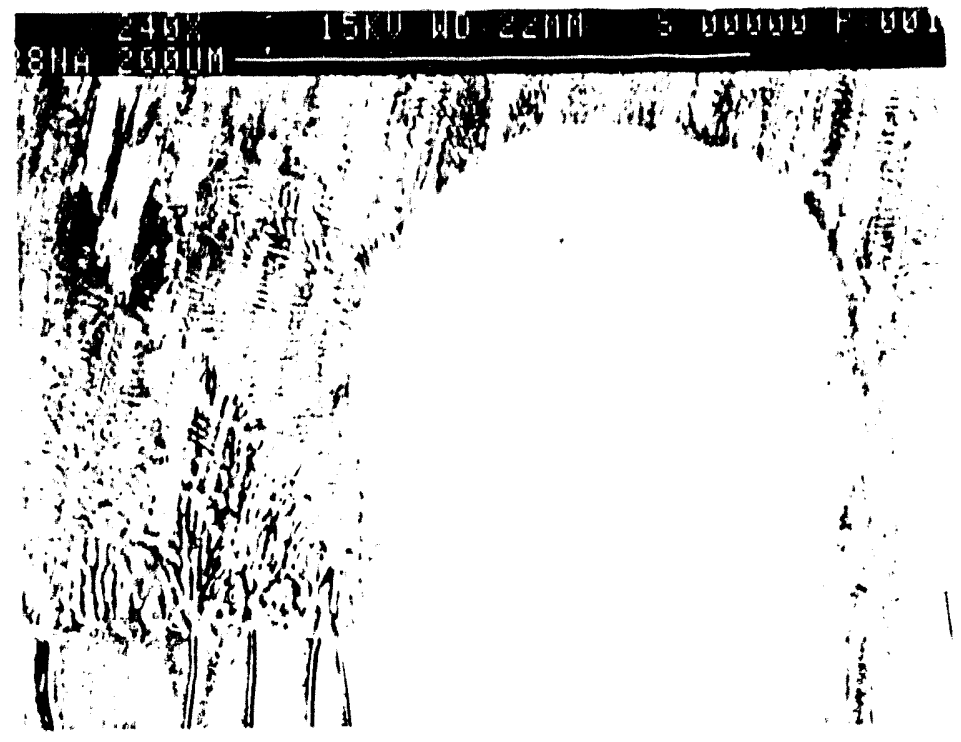

b)

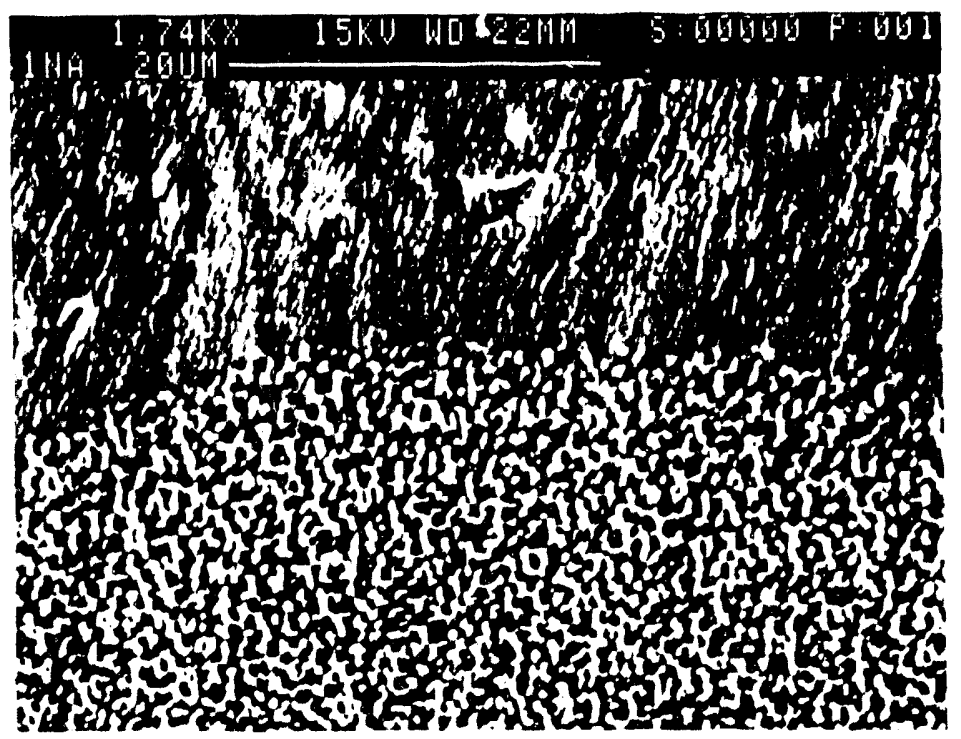

Figure 6. The SEM micrographs at the growth front of three phatse eutectic and $\gamma$ dendrite in Exp.\# 95 (Al 2.3.5(I) - $3.2 \mu \mathrm{m} / \mathrm{s})$. a) low magnification at the interface, b) at dendrite tip, 


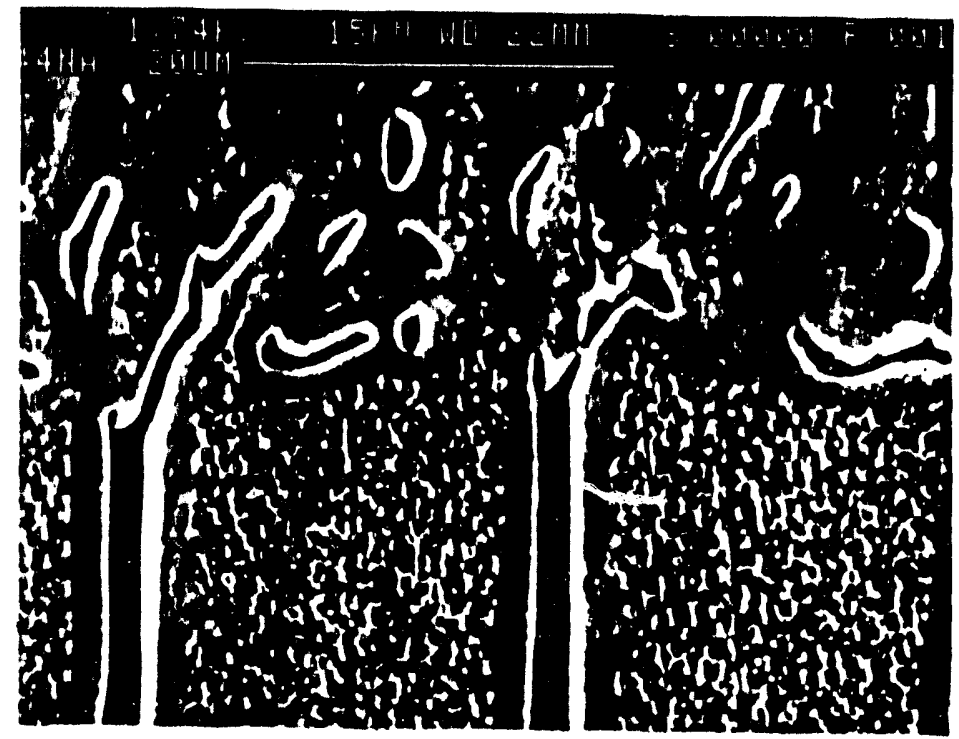

Figure 6. (continued) and c) at three phase eutectic between $\gamma$ dendrites 
a)

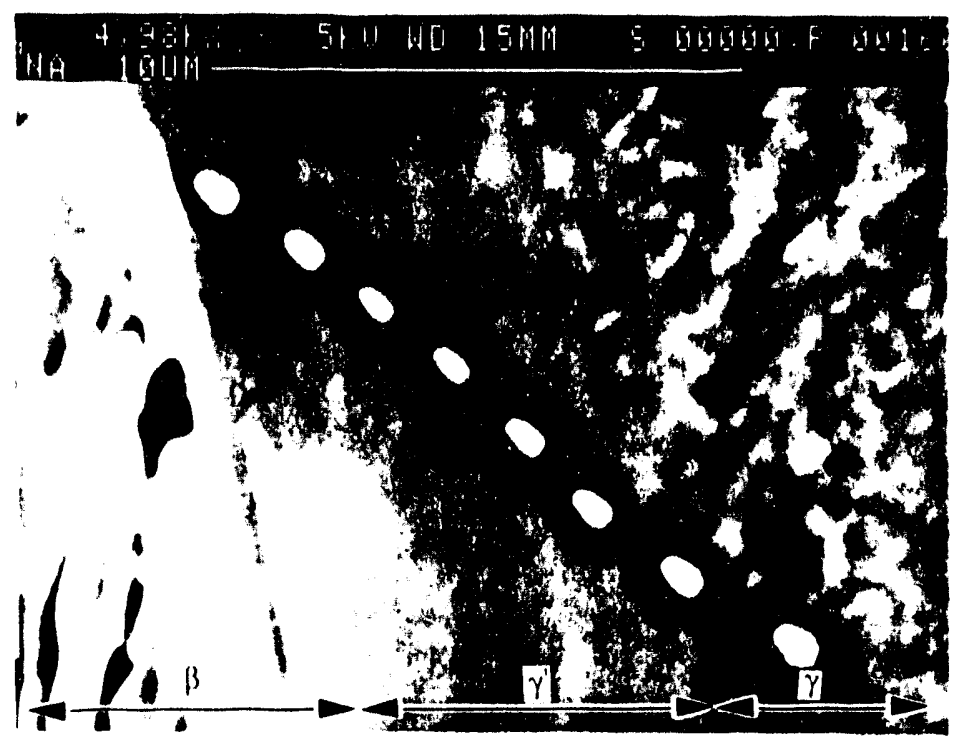

b)

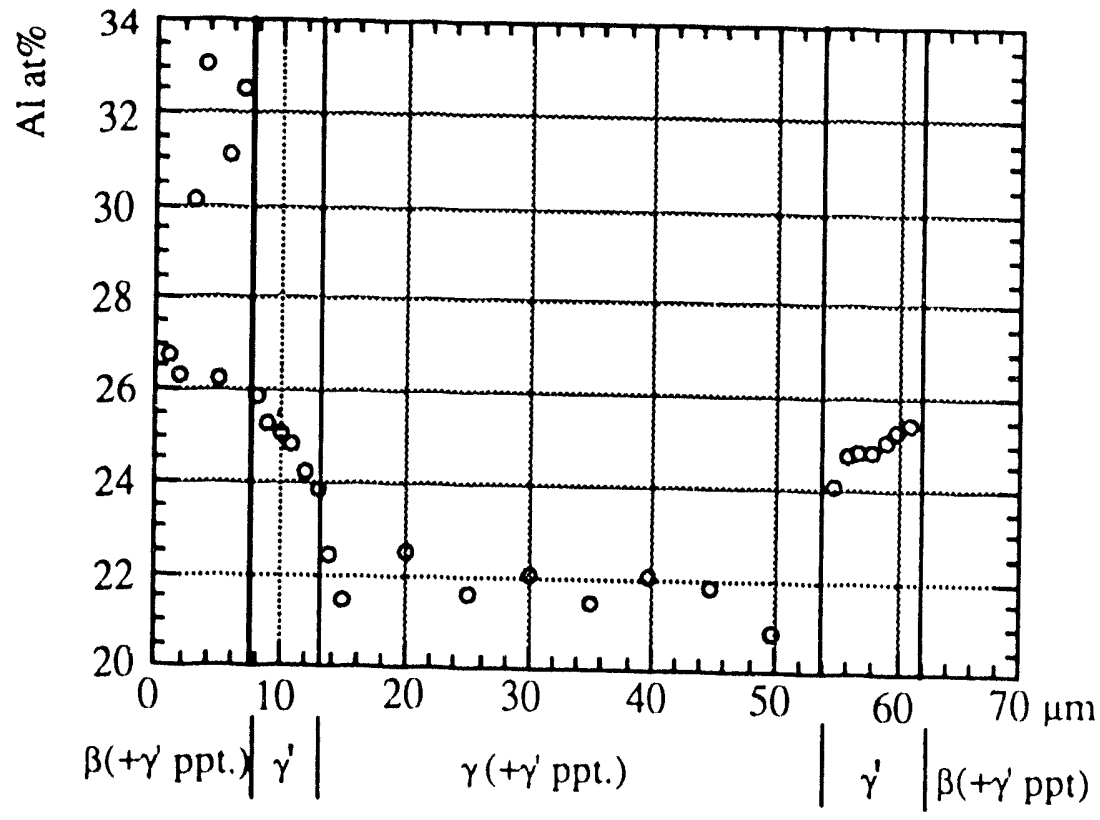

Figure 7. Composition analysis on three phases. a) The burn marks show the positions of analysis on the $\gamma^{\prime}$ phase and $b$ ) compositions in the $\beta, \gamma^{\prime}$, and $\gamma$ phase regions 
a)

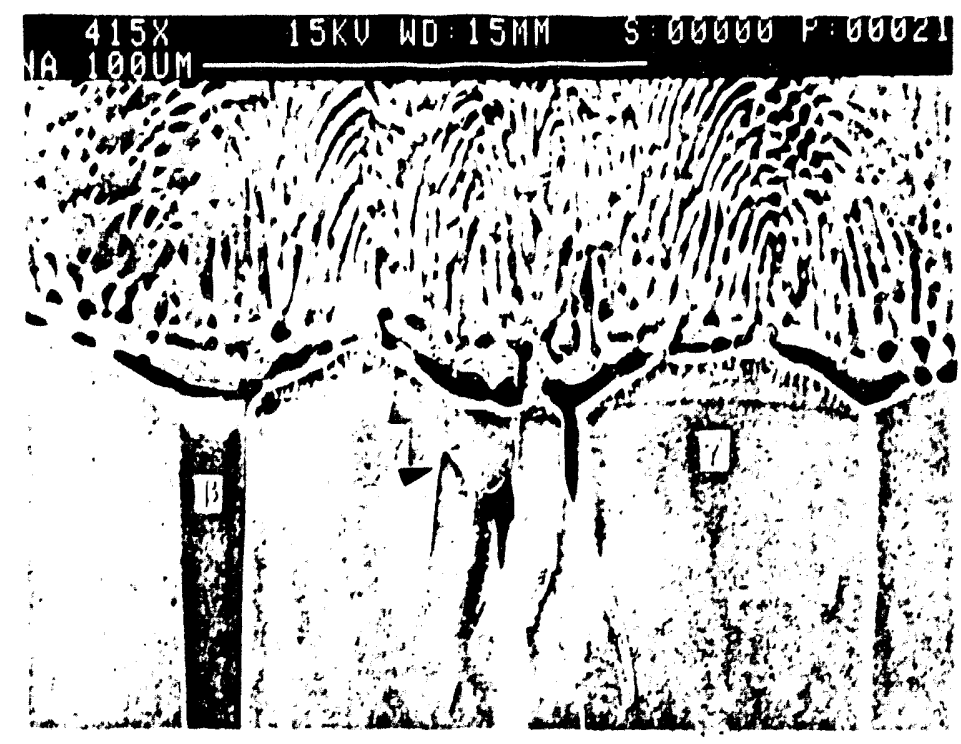

h)

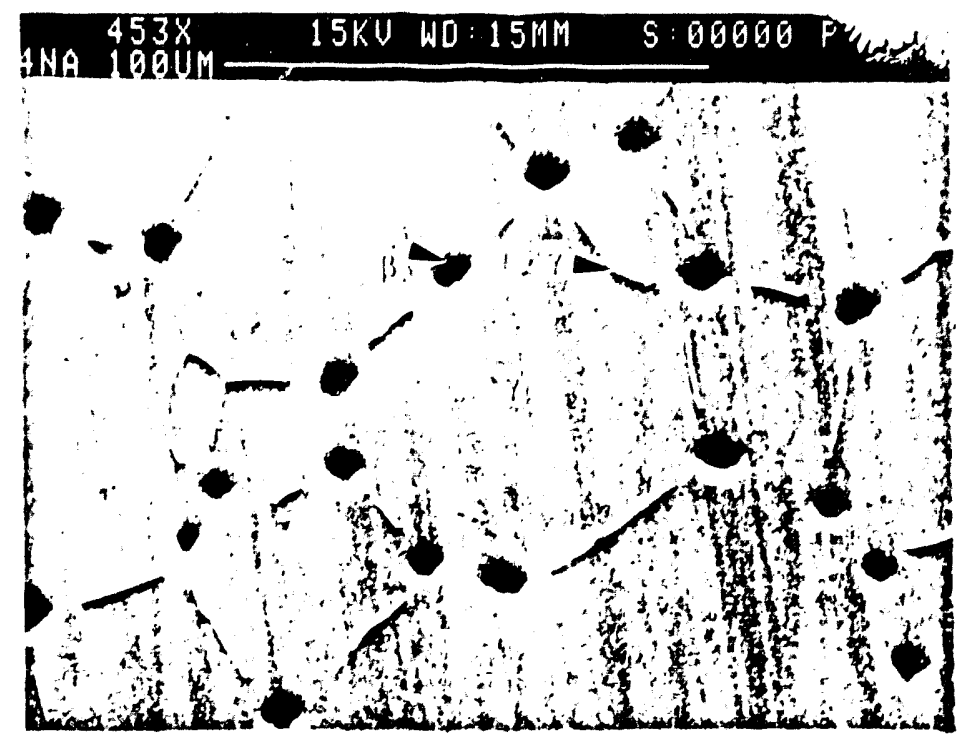

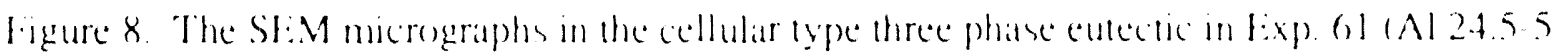
$\mu \mathrm{m} / \mathrm{s})$ a) longitudinal and b) tramsverse views 
The initial microstructural analysis and EDS analysis indicateded that three distinct phases $\left(\gamma+\gamma^{\prime}+\beta\right)$ were formed at the same growth front in both the 3P-L and 3P-R(c) eutectics. Later work with faster quench rates found that this was true only in the $3 \mathrm{P}-\mathrm{R}(\mathrm{c})$ case.

\section{Impurity effect}

Microstructural analysis revealed a strong occurrence of 3-phase eutectic structures, 3P$\mathrm{L}$ and 3P-R(c), in the two alloys, $\mathrm{Al} 23.5(\mathrm{I})$ and $\mathrm{Al}$ 24.5(I). The results of the Al 23.5(I) alloy were not consistent with the results of the $\mathrm{Al} 23$ and 24 alloys. For these reasons an impurity effect was suspected in $\mathrm{Al} 23.5(\mathrm{I})$ and $24.5(\mathrm{I})$ alloys. After the history of alloy preparation was examined on each alloy, it was found that Al 23,24,26, and 27 alloys were made of INCO $\mathrm{Ni}$ and the more recent two alloys of $\mathrm{Al} 23.5(\mathrm{I})$ and $24.5(\mathrm{I})$ were made from a the different source of $\mathrm{Ni}$ which is called $\mathrm{AO} \mathrm{Ni}$. The impurity levels on the INCO and $\mathrm{AO} \mathrm{Ni}$ were obtained from MPC (Metal Preparation Center) using two different mass spectroscopy techniques, as shown in Table 2. It appears that the INCO Ni was more pure than the AO Ni. However, the differences found in Table 2 could be due in part to the different mass spectrometry techniques used on the two samples.

In order to be consistent in alloy preparation, the alloys of $\mathrm{Al} 23.5$ and 24.5 were prepared again using the INCO Ni which was used in the alloys of Al 23, 24, 25, 26, and 27. The newly prepared Al 23.5 and 24.5 alloys, which were made of INCO Ni, are called Al 23.5(II) and $\mathrm{Al} 24.5$ (II) alloy, and the old alloys, which were made of $\mathrm{AO} \mathrm{Ni}$, are called $\mathrm{Al}$ 24.5(I) and Al 23.5(I) alloy. The results of Al 24.5(II) alloy showed the 3P-L, 3P-R(c), and 2P-R(c) eutectics, and the 2P-R(c) and 3P-R(c) eutectics were found at low rates in Al 23.5(II) alloy. The morphologies in Al 24.5(II) alloy were similar to the results in Al 24.5(I) alloy. However, the results of $\mathrm{Al} 23.5(\mathrm{I})$ and $\mathrm{Al}$ 23.5(II) alloys were different, but those of $\mathrm{Al}$ 23.5(II) alloy were consistent to the results of Al 23 and 24 alloys. These results indicated that the alloy $\mathrm{Al} 23.5$ (I) had something wrong with it. 
Table 2. Impurity analysis in INCO Ni and $\mathrm{AO} \mathrm{Ni}+$

\begin{tabular}{|c|c|c|c|c|}
\hline Impurity & INCO Ni* & AO Ni** & Unit & Method \\
\hline \hline $\mathrm{C}$ & 42 & 51 & ppm wt.\% & Combustion \\
$\mathrm{O}$ & 29 & 26 & & \\
$\mathrm{H}$ & 0 & 2 & & \\
$\mathrm{~N}$ & 2 & 0 & & \\
\hline $\mathrm{F}$ & $<0.1$ & 12 & ppm at.\% & Mass Spec. \\
$\mathrm{Al}$ & $<0.03$ & 3 & & \\
$\mathrm{Si}$ & $<1$ & 30 & & *INCO Ni by \\
$\mathrm{P}$ & $<7$ & 7 & & Spark Source \\
$\mathrm{S}$ & $<0.1$ & 8 & & \\
$\mathrm{Cl}$ & 1 & 45 & & **AO Ni by \\
$\mathrm{Sc}$ & 0.03 & 2 & & Laser Source \\
$\mathrm{K}$ & $<0.1$ & 1.2 & & \\
$\mathrm{Fe}$ & 0.9 & 3.8 & & \\
$\mathrm{Cr}$ & 0.29 & $<0.1$ & & \\
$\mathrm{Cu}$ & $<1$ & 1.7 & & \\
$\mathrm{Sn}$ & $<1$ & 8 & & \\
$\mathrm{Nb}$ & $<4$ & 5.3 & & \\
$\mathrm{~B}$ & $<0.1$ & 8.6 & & \\
$\mathrm{Mn}$ & $<0.5$ & 2.0 & & \\
$\mathrm{Mg}$ & $<0.2$ & 0.24 & & \\
\hline
\end{tabular}

+ Data from MPC(Matal Preparation Center) 
In order to investigate the impurities in each alloy, the impurities were analyzed on the Al 23, 23.5(I,II), 24, and 24.5(I,II) alloys. Tables 3 and 4 show the results of the impurity analyses by the mass spectrometric method using the laser source. The $\mathrm{C}$ and $\mathrm{N}$ were analyzed by combustion technique. The result shows that the master ingot of $\mathrm{Al} 23.5(\mathrm{I})$ alloy was contaminated by $\mathrm{Fe}, \mathrm{Cr}, \mathrm{Mn}, \mathrm{Si}$, and $\mathrm{Nb}$. This alloy looks to be contaminated by a stainless steel because the major constituents are $\mathrm{Fe}, \mathrm{Cr}, \mathrm{Si}$, and $\mathrm{Mn}$, and $\mathrm{Nb}$ is an additive in some stainless steels. It is expected that a contaminated crucible was used or some stainless scraps were accidentally included in Al 23.5 (II) alloy. These impurity analyses also indicate that there is no significant difference in the impurity levels between INCO Ni and AO Ni because the results of $\mathrm{Al} 24.5$ (I) and (II) are almost the same.

Comparing the results of $\mathrm{Al} 23.5(\mathrm{I})$ and $\mathrm{Al} 23.5$ (II) in Table 1 it is seen that the impurities of $\mathrm{Al} 23.5$ (I) change the eutectic microstructure to the 3P-L form from a mixed form. At first, it was thought that maybe the impurities were producing a 3-phase ternary eutectic. This ternary eutectic is thought to be induced by the $2000 \mathrm{ppm}$ level of impurities. However, these ppm levels of $\mathrm{Fe}$ and $\mathrm{Cr}$ are too small to form the equilibrium ternary eutectic phases because it has been reported that at least 3-5 at.\% is necessary to form the equilibrium ternary eutectics with $\mathrm{Cr}$ or $\mathrm{Fe}[14,15]$. Also, there still appears to be a three phase eutectic morphology in the low impurity alloy of $\mathrm{Al} 23.5$ (II) where it is a $3 \mathrm{P}-\mathrm{R}(\mathrm{c})$ type.

In order to prove that the 3P-L eutectic would be induced by impurities ( $\mathrm{Fe}$ and $\mathrm{Cr}$ ), the impurities were added to the sample of the alloy Al 23.5(II) on purpose. A sheet of 304 stainless steel, which includes 71 at.\% Fe and 18 at\% $\mathrm{Cr}$, was added as much as the alloy had about 1500 ppm Fe and 500 ppm $\mathrm{Cr}$. Ternary lamellar type eutectic (3P-L) was obtained at 3.2 $\mu \mathrm{m} / \mathrm{s}$ and it was the same structure to the elitectic structure of the contaminated old alloy $\mathrm{Al}$ 23.5 (I). These results shows that the low levels of $\mathrm{Cr}$ and Fe impurities do affect the type of eutectic morphology which occurs. 
Table 3. Impurity analysis by Mass spectometric method by Laser source

\begin{tabular}{|c|c|c|c|c|c|c|c|c|c|c|c|}
\hline \multirow[t]{2}{*}{ Alloy } & \multirow[t]{2}{*}{$V, \mu \mathrm{m} / \mathrm{s}$} & \multirow[t]{2}{*}{ Exp\# } & \multirow{2}{*}{ Position } & \multicolumn{8}{|c|}{ Impurity (ppm at\%) } \\
\hline & & & & $\mathrm{Fe}$ & $\mathrm{Cr}$ & $\mathrm{Si}$ & $\mathrm{Mn}$ & $\mathrm{Mg}$ & $\mathrm{Nb}$ & $\mathrm{B}$ & $\underline{\underline{s}}$ \\
\hline $24.5(1)$ & 3.2 & 98 & Liq & 7.0 & 0.36 & 24 & $<0.08$ & 15 & $<0.05$ & 4.1 & $<10$ \\
\hline \multirow[t]{4}{*}{$24.5(\mathrm{II})$} & \multirow[t]{3}{*}{3.2} & \multirow[t]{3}{*}{118} & Liq & 10 & 0.64 & $<5$ & 0.2 & 3.0 & $<0.03$ & 2.9 & $\leq 3$ \\
\hline & & & Sol(A)* & 7.5 & 0.37 & $<9.0$ & 0.19 & 4.0 & 0.14 & 0.65 & $<0.7$ \\
\hline & & & Sol(B)* & 13 & 0.63 & 15 & 0.29 & 5.0 & 0.27 & 4.9 & $<0.7$ \\
\hline & 0.8 & 117 & Liq & 11 & 0.50 & $<10$ & 0.20 & 3.0 & 0.14 & 7.5 & $<2$ \\
\hline 24 & 3.2 & 96 & Liq & 7.5 & $<0.2$ & 25 & $<0.07$ & 8.5 & $<0.05$ & 4.0 & $\leq 4$ \\
\hline \multirow[t]{3}{*}{$23.5(\mathrm{I})$} & \multicolumn{3}{|c|}{ Master Ingot } & 2800 & 1200 & $\leq 100$ & 65 & 0.37 & 29 & 1.5 & $<0.4$ \\
\hline & \multirow[t]{2}{*}{3.2} & \multirow[t]{2}{*}{92} & Liq & 1400 & 660 & 90 & 39 & 17 & 22 & 1.2 & 12 \\
\hline & & & Sol & 1100 & 550 & 90 & 25 & 12 & 17 & 2.4 & $<6$ \\
\hline $23.5(\mathrm{II})$ & 3.2 & 111 & Liq & 11 & 0.85 & $<20$ & 0.24 & 8.0 & $<0.05$ & 2.2 & $<20$ \\
\hline \multirow[t]{2}{*}{23} & \multirow[t]{2}{*}{3.2} & \multirow[t]{2}{*}{104} & Liq & 9.0 & 0.45 & 20 & 1.5 & 6.0 & $<0.05$ & 2.8 & $<2$ \\
\hline & & & Sol & 7.5 & $<0.4$ & 20 & 1.2 & 9.0 & $<0.05$ & 1.6 & $<1$ \\
\hline
\end{tabular}

* Sol(A) is the center region and sol(B) is the edge region of the directionally solidified rod $1 \mathrm{~mm}$ below the solid-liquid interface.

Table 4. $\mathrm{C}, \mathrm{O}$, and $\mathrm{N}$ analysis by combustion technique

\begin{tabular}{|c|c|c|c|c|c|c|}
\hline Alloy & \multirow{2}{*}{$\mathrm{V}(\mu \mathrm{m} / \mathrm{s})$} & \multirow{2}{*}{ Exp.\# } & Position & \multicolumn{3}{|c|}{ Impurity (ppm wt.\%) } \\
\cline { 4 - 7 } & & & & $\mathrm{O}$ & $\mathrm{N}$ & $\mathrm{C}$ \\
\hline \hline $24.5(\mathrm{I})$ & 3.2 & 98 & Liquid & 24 & $<1$ & 42 \\
\hline 24 & 3.2 & 96 & Liquid & 20 & $<1$ & 47 \\
\hline $23.5(\mathrm{I})$ & 3.2 & 92 & Liquid & 19 & $<1$ & 51 \\
\cline { 4 - 7 } & & & Solid & 17 & $<1$ & 46 \\
\hline 23 & \multirow{2}{*}{3.2} & \multirow{2}{*}{104} & Liquid & 27 & $<1$ & 51 \\
\cline { 4 - 7 } & & & Solid & 4 & $<1$ & 48 \\
\hline
\end{tabular}


An interesting result was found in contaminated alloy of $\mathrm{Al}$ 23.5(I). The usual solidification sequence at low rates in hypo eutectic alloys was the successive formation of $\gamma$ planar, $\gamma^{\prime}$ planar, and eutectic structure. In Al 23.5(I) alloy, however, the sequence did not include the $\gamma^{\prime}$ planar, but went directly from $\gamma$ to the eutectic before $\gamma^{\prime}$ nucleates. It means the impurities cause the liquid composition at the growth front to follow the $\gamma$ liquidus all the way to the eutectic temperature without nucleation of the pure $\gamma^{\prime}$ phase. Figure 9 shows the metastable $\gamma$ liquidus line is extrapolated to the eutectic region. It appears that these impurities suppress the formation of $\gamma^{\prime}$ until eutectic forms.

\section{Velocity effect}

The interdendritic eutectics at high growth rates $(61$ and $25 \mu \mathrm{m} / \mathrm{s})$ were always the $3 \mathrm{P}-\mathrm{L}$ eutectic in all alloy compositions, as shown in Figure 10. Initial analysis in the eutectic of 61 $\mu \mathrm{m} / \mathrm{s}$ presented in Paper I indicated that this eutectic was $\gamma^{\prime}+\beta$ eutectic and $\gamma$ was precipitated in the $\gamma^{\prime}$ phase. This explanation caused a problem in the $\gamma^{\prime}+\beta$ eutectic at low rates because the $\gamma^{\prime}$ phase in $\gamma^{\prime}+\beta$ eutectic of low rates did not include the $\gamma$ precipitates.

After the 3P-L eutectic was found in contaminated alloy of $\mathrm{Al} 23.5(\mathrm{I})$ at low rates, it was suggested that the eutectic of $61 \mu \mathrm{m} / \mathrm{s}$ might be the same kind of three phase eutectic(3P-L) which was thought to be the $\gamma^{\prime}+\beta$ eutectic with $\gamma$ precipitates in the $\gamma^{\prime}$ phase of the eutectic in Paper I.

\section{Composition Variations and Eutectic Morphologies}

Even though there are no impurities (low levels of $\mathrm{Cr}$ and $\mathrm{Fe}$ ), three phase eutectics(3P. $\mathrm{L}$ and 3P-R(C)) were formed at low rates in $\mathrm{Al} 24.5(\mathrm{I}), 24.5(\mathrm{II})$, and 23.5(II) alloys. The 3PL eutectic, which is the same structure in the contaminated alloy ( $\mathrm{Al} 23.5(\mathrm{I})$ ), was found at 0.8 $\mu \mathrm{m} / \mathrm{s}$ in Al 24.5(II) alloy, as shown in Figure 4. Figure 11 shows that the 3P-L eutectic forms in the center region of the rod and $3 P-R(c)$ and $2 P-R(c)$ eutectics form in the edge region at 


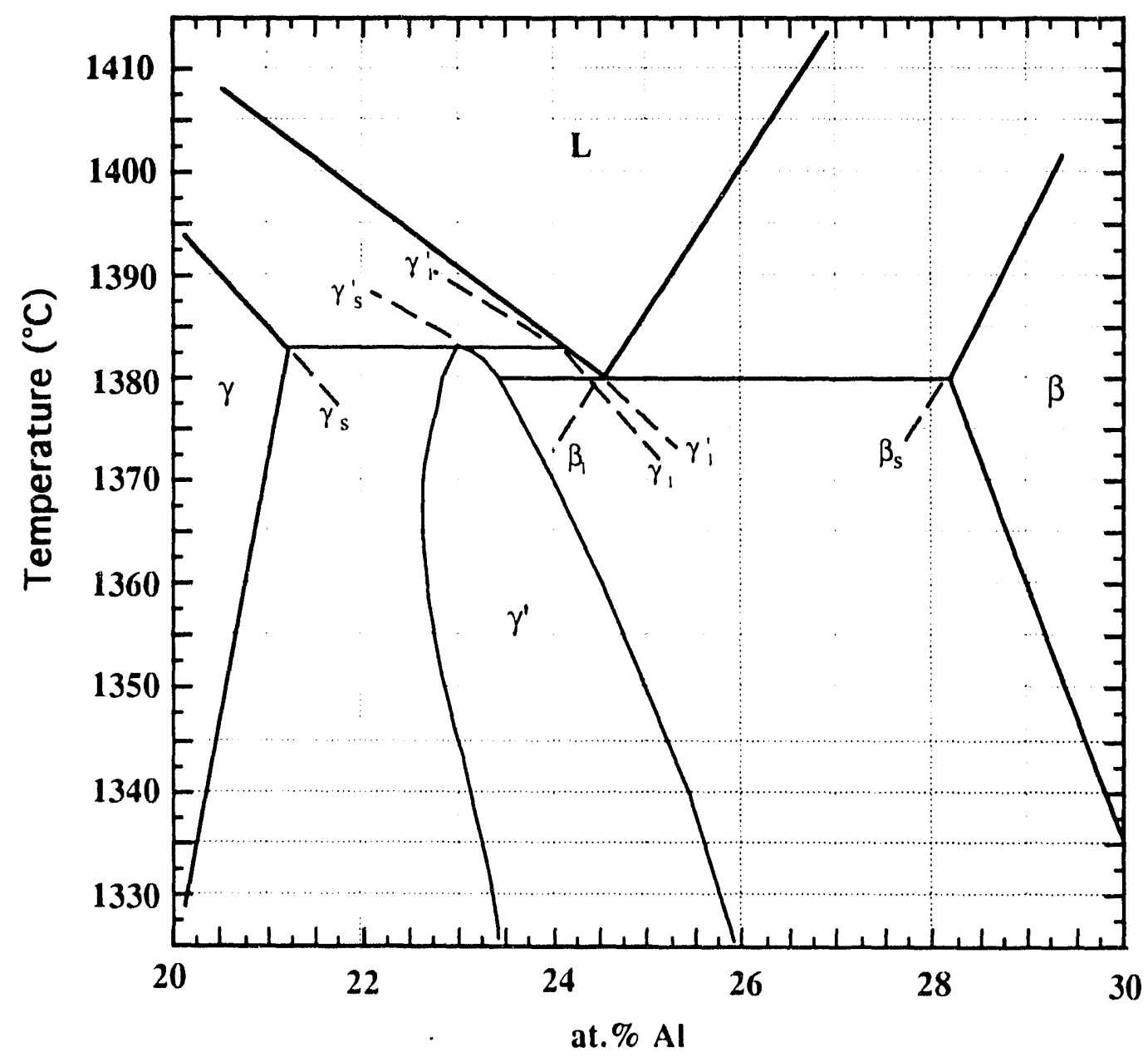

Figure 9. Equilibrium and metastable phase diagram near $\mathrm{Ni} 3 \mathrm{Al}$ compositions from Figure 13 in Paper I 
a)

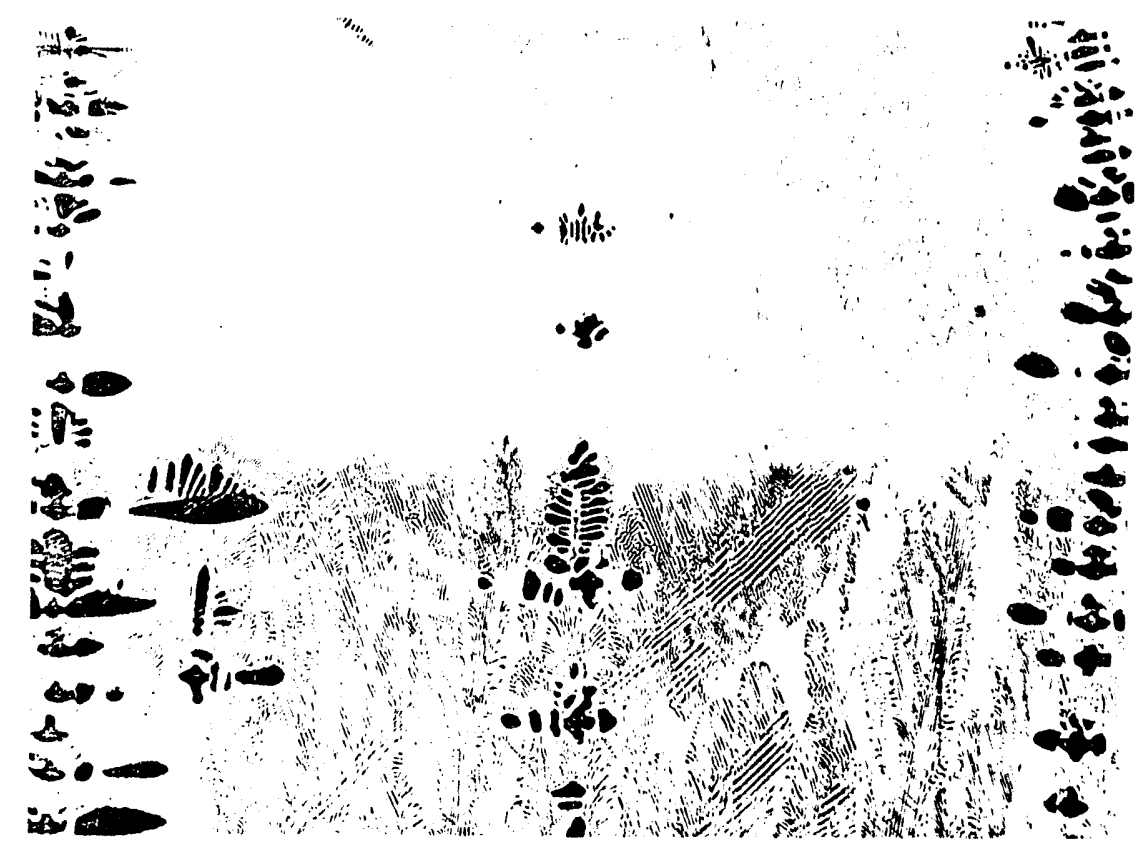

b)

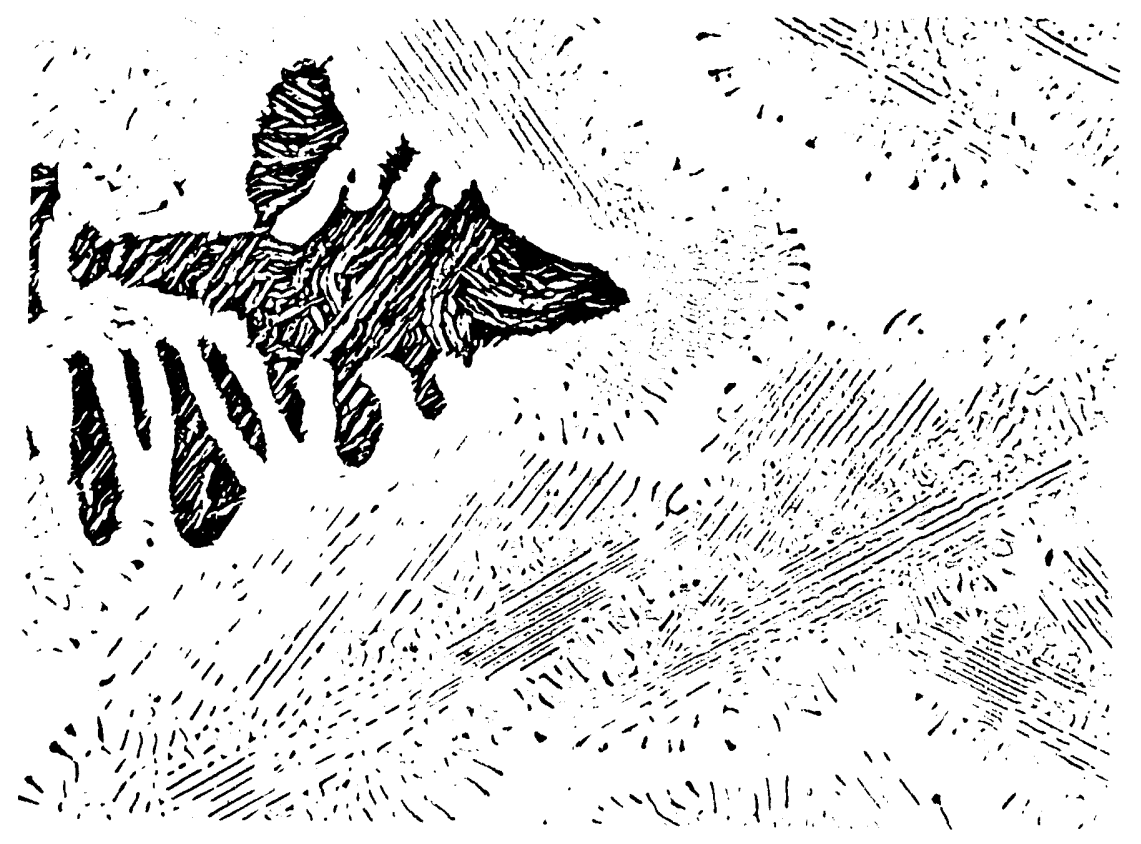

Figure 10. Three phase eutectic between dendrites at $61 \mu \mathrm{m} / \mathrm{s}$ in $\mathrm{Al} 25$ alloy (Exp. 8).

a) Longitudinal view, $40 \mathrm{x}$ and b) transverse view, $200 \mathrm{x}$ 
a)

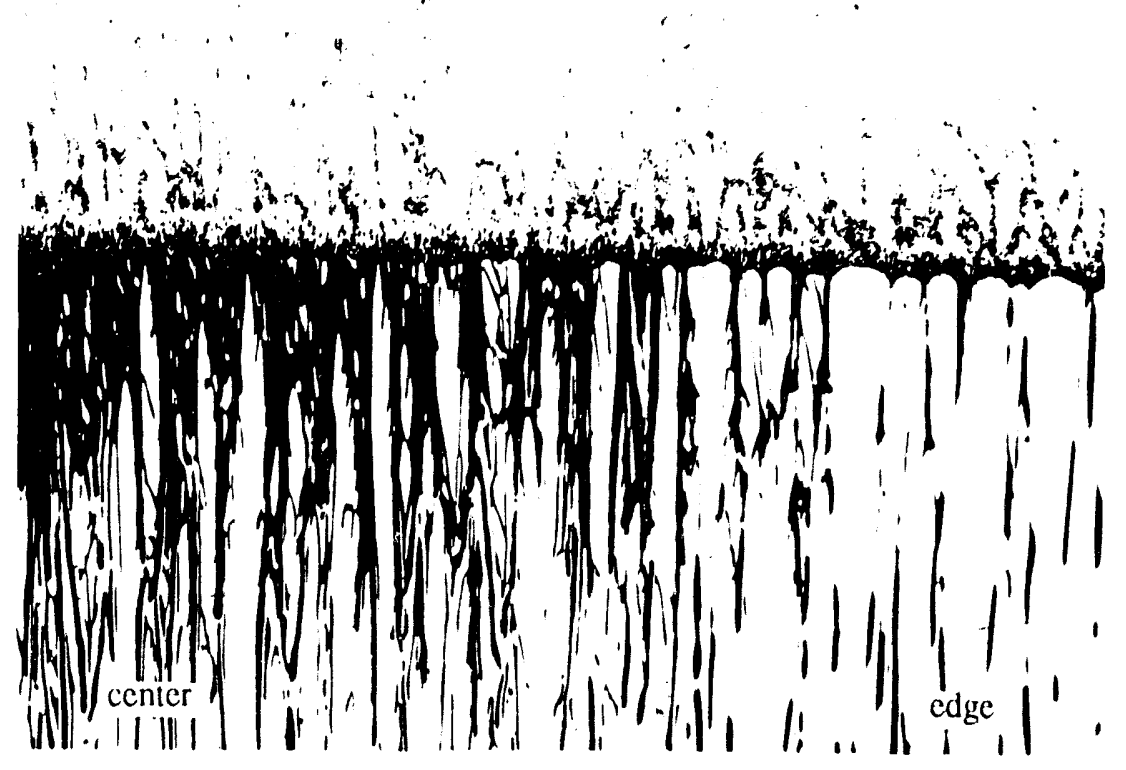

b)

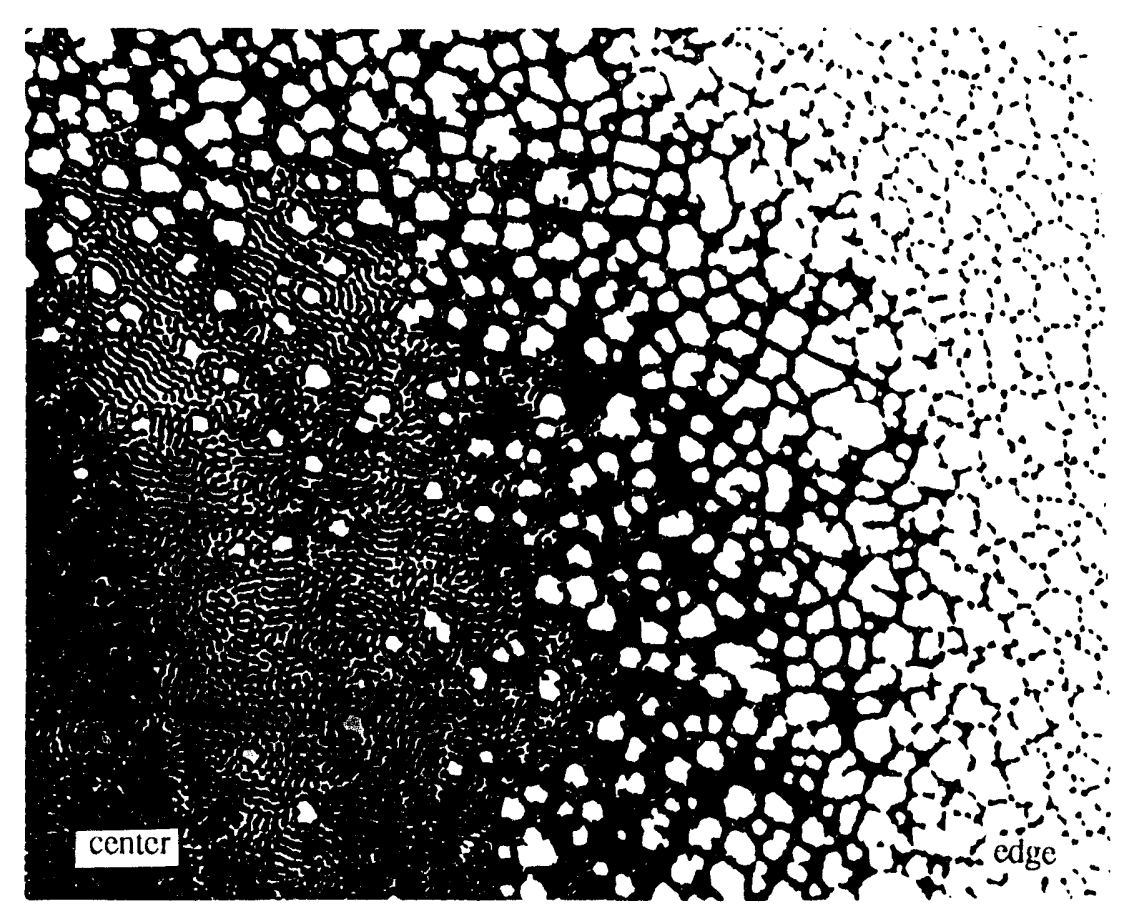

Figure 11. Optical micrographs in Al 24.5(II) alloy, 50x. a) Longitudinal and b) transverse views in Exp.\# $118(3.2 \mu \mathrm{m} / \mathrm{s})$, 


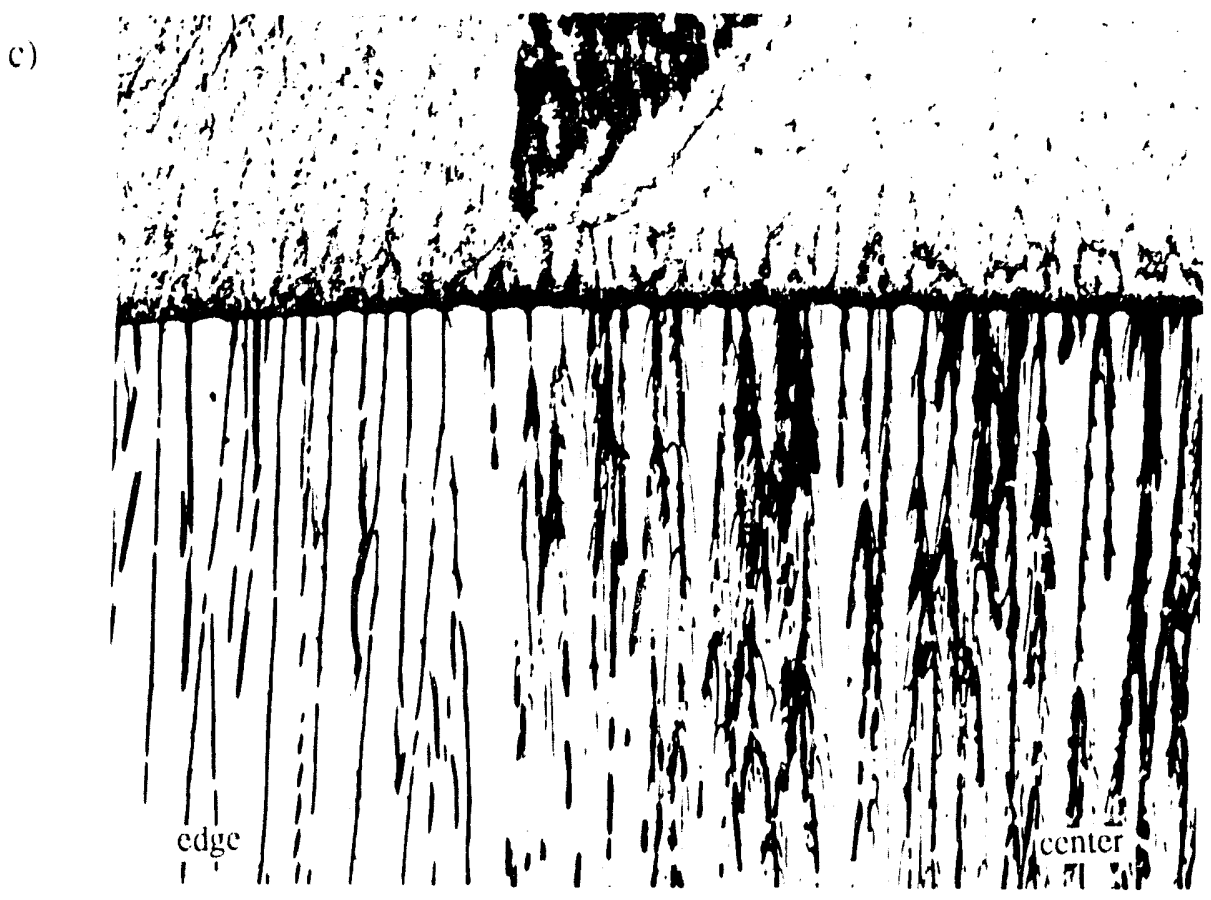

d)

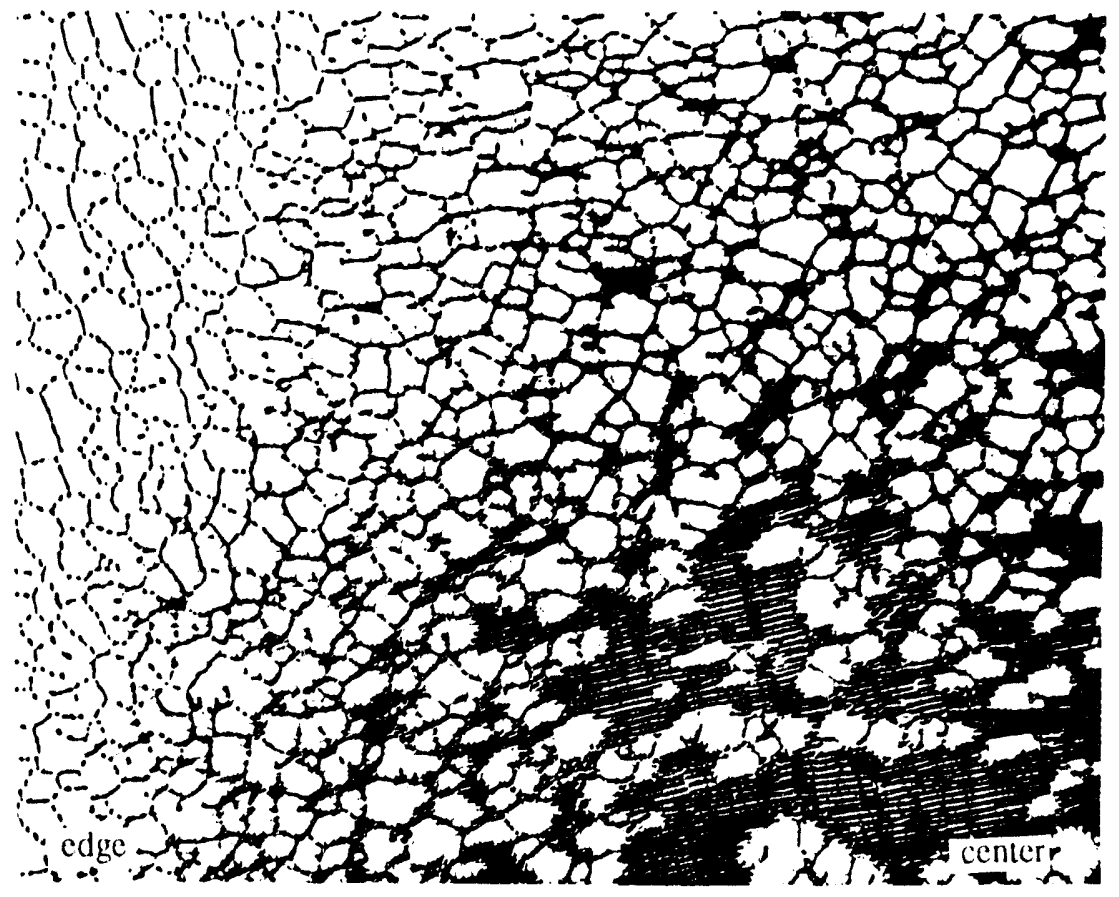

Figure 11. (continued) c) longitudinal and d) transverse views in Exp.\#110 $(5 \mu \mathrm{m} / \mathrm{s})$, 


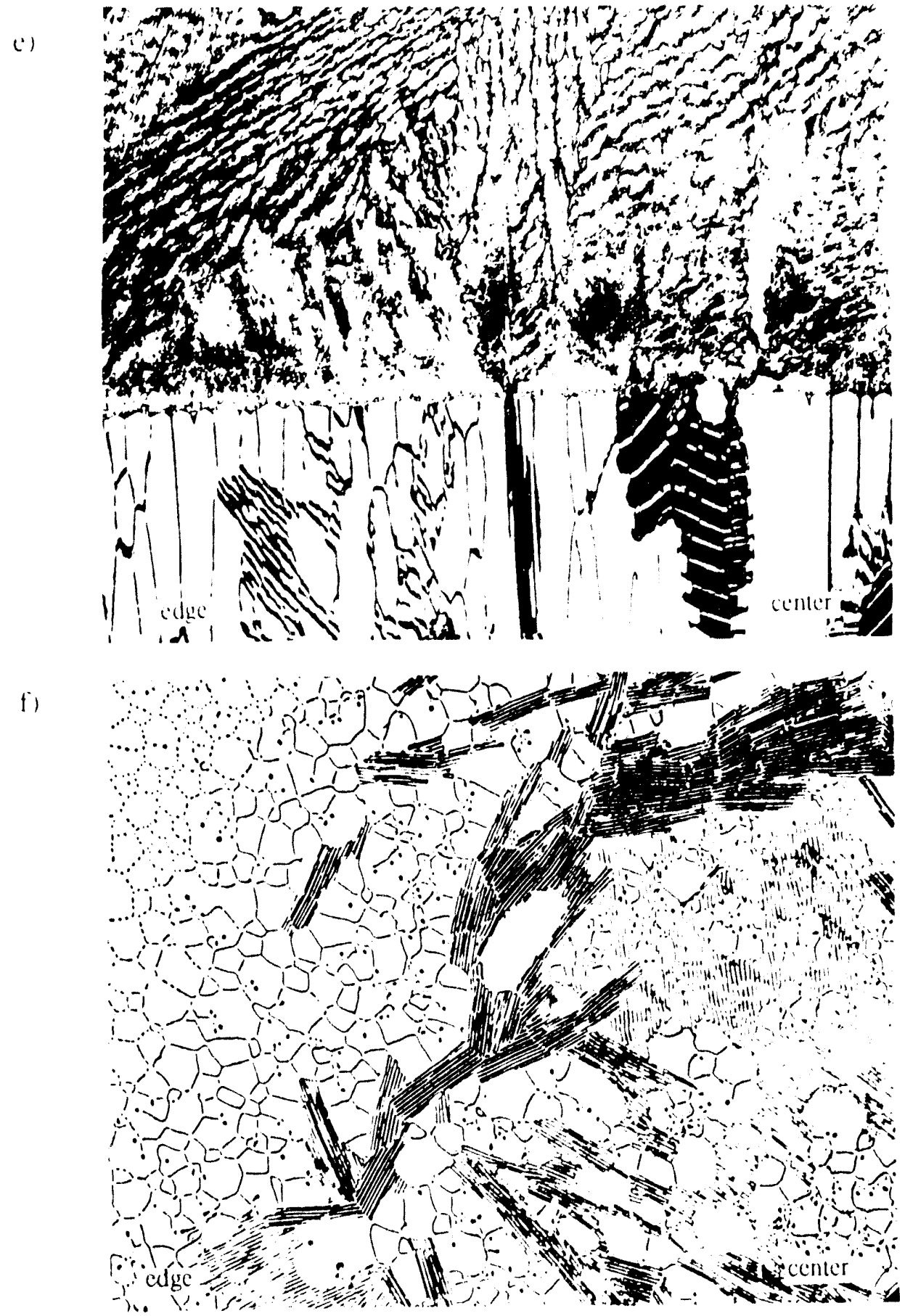

Figure 11. (continued) e) longitudinal and f) transverse views in Exp. \#116(10 $\mu \mathrm{m} / \mathrm{s})$ 
3.2 .5 , and $10 \mu \mathrm{m} / \mathrm{s}$ in alloy $\mathrm{Al} 24.5$ (II). These results indicate that a small composition shift may cause different types of eutectic structure. Even hough the solid-liquid interface is planar, the edge side of the rod is a little lower than the center. In order to determine if there were impurity variations between the $3 \mathrm{P}-\mathrm{L}$ and $3 \mathrm{P}-\mathrm{R}(\mathrm{c})$ eutectics, the impurities of the center(lamellar) and edge(cellular) region in Figure 11(b) were analyzed. As shown in Table 3, there were no significant differences in impurities between these two structures.

The 2P-R(c) and 3P-R(c) eutectics were found at 3.2 and $5 \mu \mathrm{m} / \mathrm{s}$ in $\mathrm{Al} 24.5(\mathrm{I})$ alloy, as shown in Figure 12. Figures 13(a) and (b) show the 3P-L and 3P-R(c) eutectic structures, which has just started to form at $f s=0.47$ and the $3 P-R(c)$ eutectic structure at $f s=0.60$ at 0.8 $u m / s$ in $\mathrm{Al} 24.5$ (I) alloy. This result indicates that there is composition variations according to the solidification fractions $\left(f_{\mathrm{S}}\right)$ by convection and it may change the eutectic morphology. It is also thought that there is a little difference in composition between $\mathrm{Al}$ 24.5(I) and $\mathrm{Al}$ 24.5(II) alloys. The 3P-L eutectic was formed at $f_{S}$ as low as 0.2 in Al 24.5(II), whereas Figure 13 shows that the eutectic does not form in the Al 24.5(I) alloy until $\mathrm{fs}=0.47$ at $0.8 \mu \mathrm{m} / \mathrm{s}$. This result indicates that the $\mathrm{Al} 24.5$ (l) alloy has a little lower $\mathrm{Al}$ than the $\mathrm{Al} 24.5$ (II) alloy because the initial phase was the $y$ planar at low rates in the Al 24.5(I) alloy. It is expected that the composition of $\mathrm{Al} 24.5(\mathrm{I})$ is a little lower than the eutectic composition and that of $\mathrm{Al} 24.5$ (II) is at the eutectic composition or slightly higher

The eutectic formed at the end part of solidification after $\gamma$ and $\gamma$ planar growth at low rates in hypo eutectic alloys (AI 22, 23.5(II), 24 alloys). Several kinds of eutectics, 2P-L, 2P$R(c p), 2 P-R(c), 3 P-R(c)$ occured, as shown in Figure 14. It was interesting to find the $\beta$ layer at the end of $\gamma^{\prime}$ planar and the beginning of the eutectic in the same manner as in Figure 13(a). This result indicates that the $\gamma$ planar interface is undercooled to the hyper eutectic composition side to form the $\beta$ phase and then the eutectic forms.

The experimental results show that all the eutectic structures must be formed around the eutectic composition but small composition variations might result in several kinds of eutectic 
a)

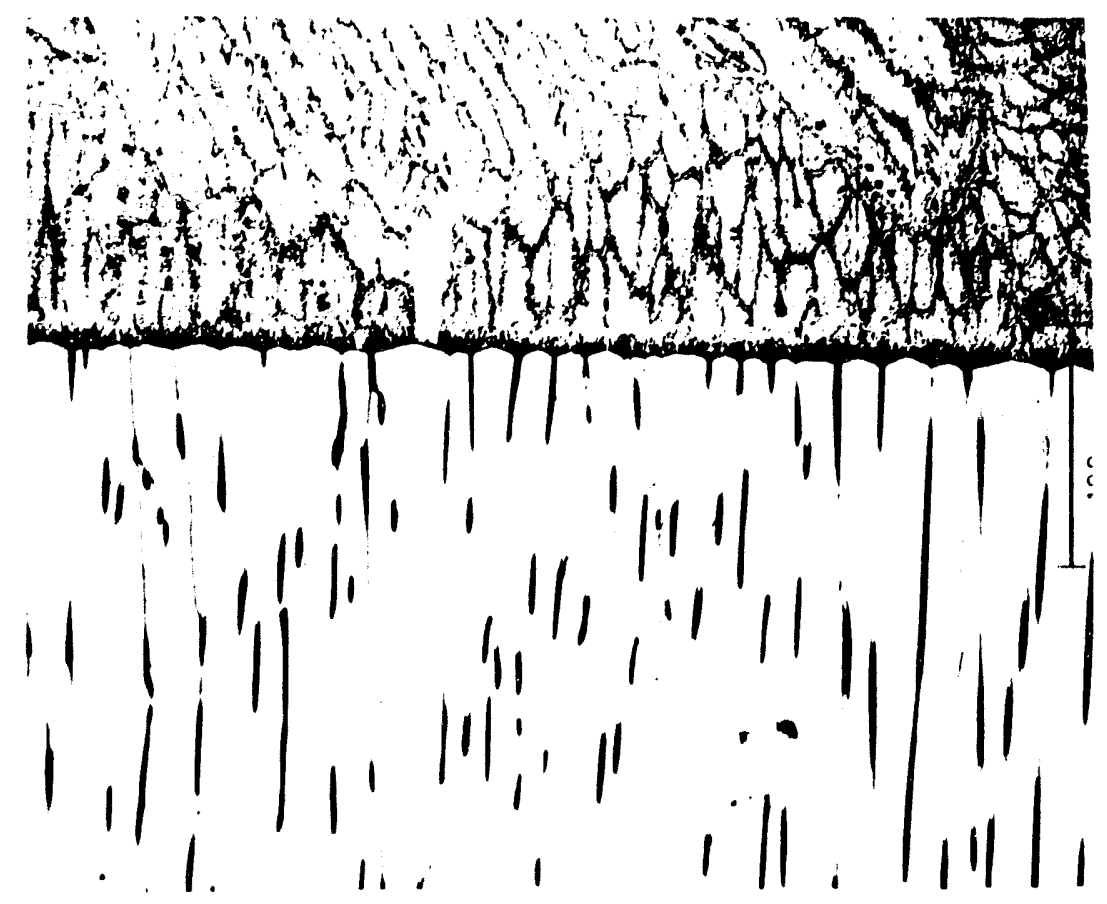

b)

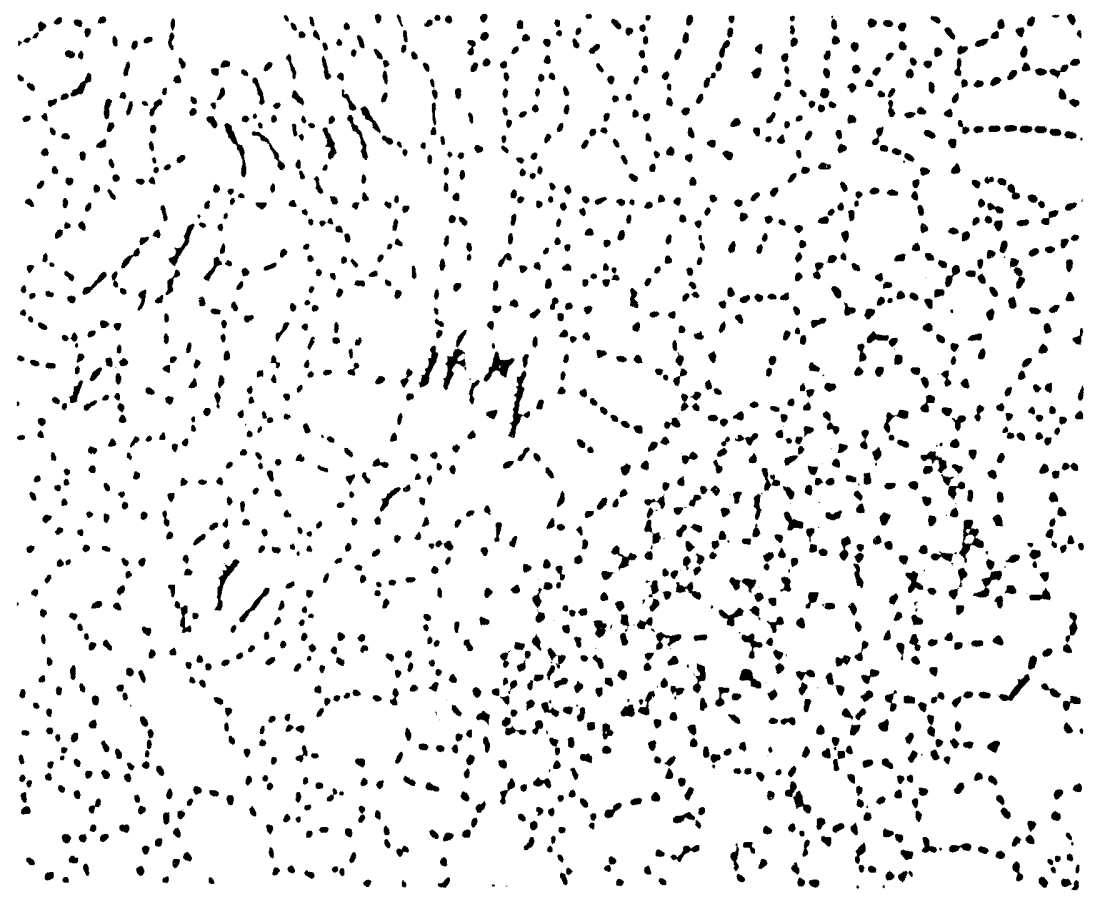

Figure 12. Optical micrographs of the solid-liquid interfaces in Al 24.5 (I) alloy, 50x. a) Longitudinal and b) transverse views in Exp.\# $59(3.2 \mu \mathrm{m} / \mathrm{s})$, 
c)

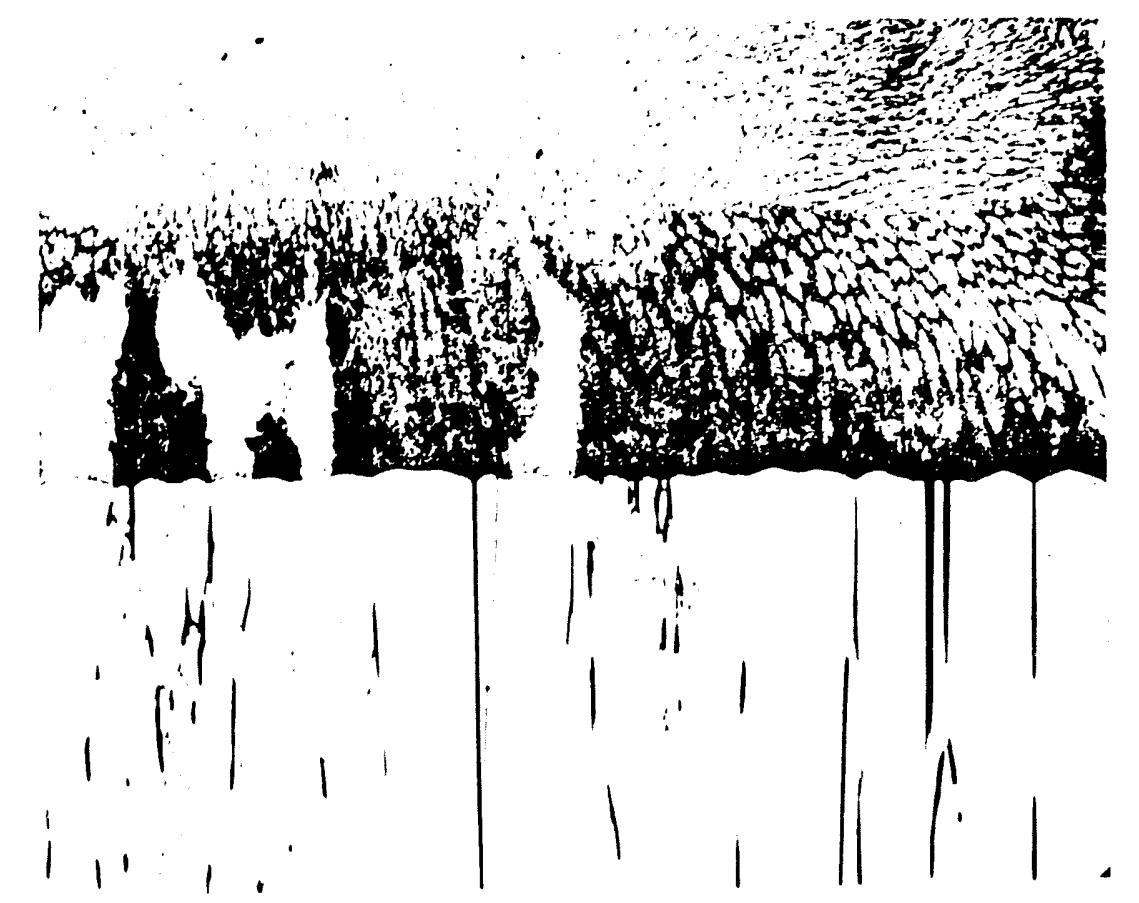

d)

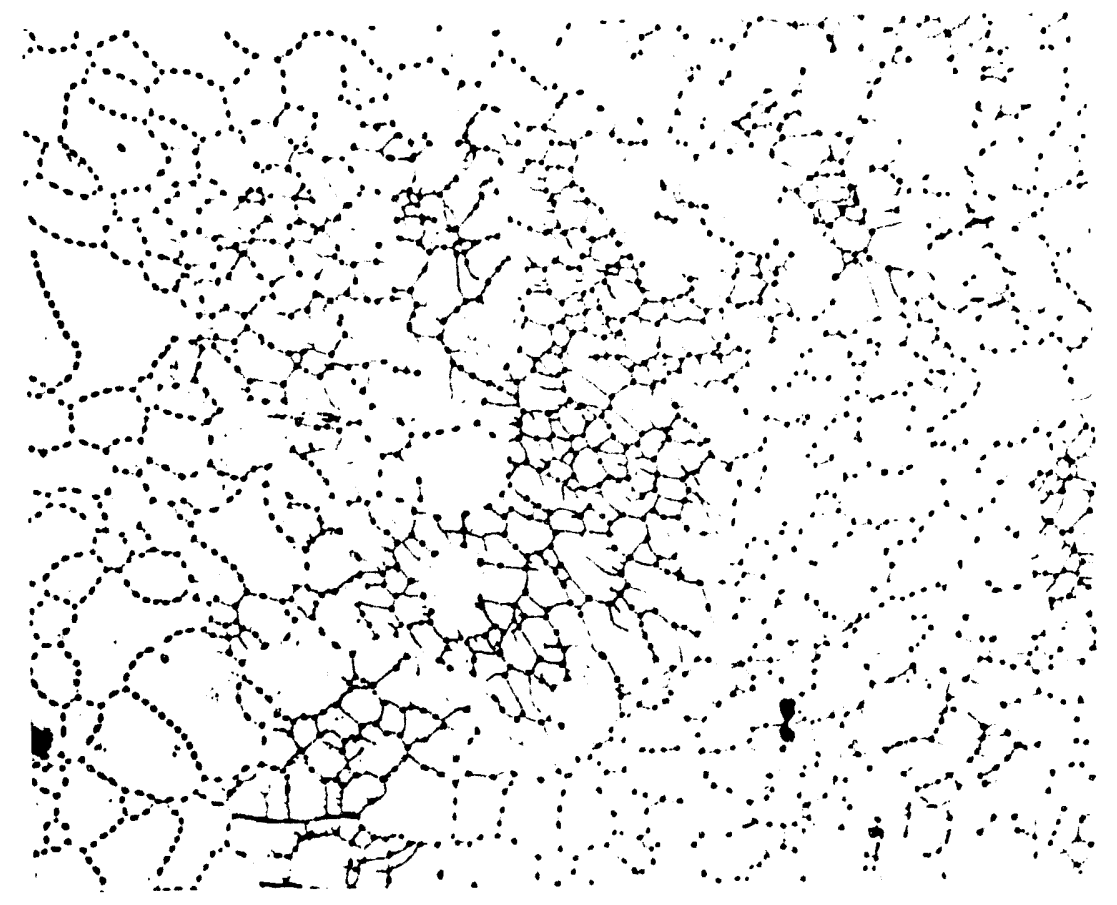

Figure 12. (continued) and c) longitudinal and d) transverse views in Exp.\#61 $(5 \mu \mathrm{m} / \mathrm{s})$ 
a)

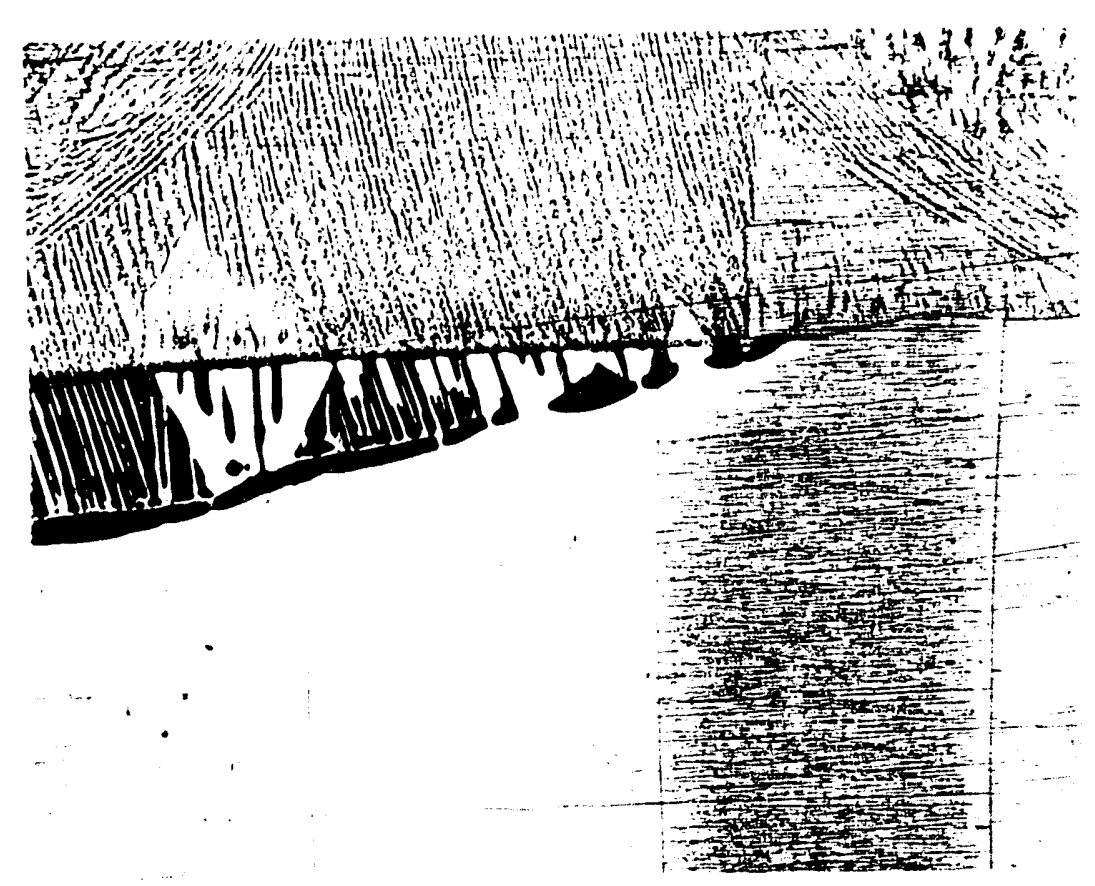

b)

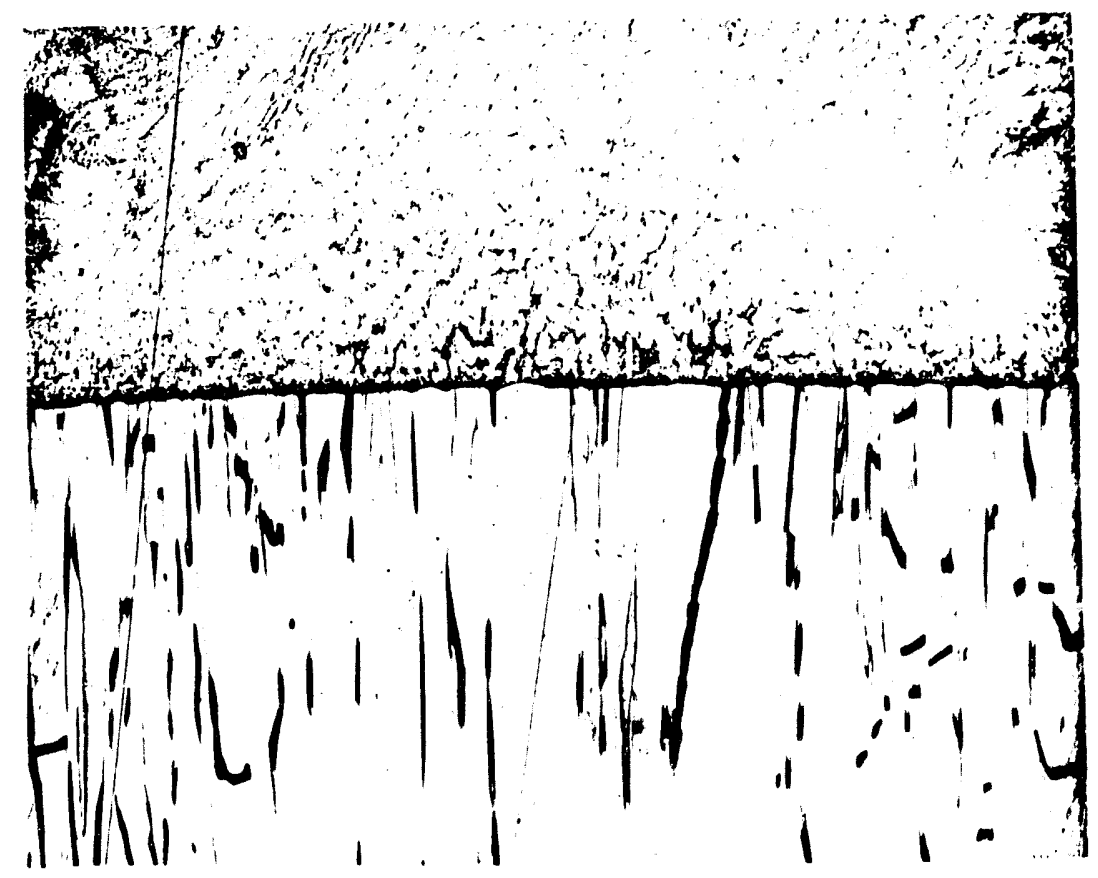

Figure 13. The eutectic structures at $(0.8 \mu \mathrm{m} / \mathrm{s}$ in $\mathrm{Al} 24.5$ (1) alloy according to the solidification fractions. 25x. a) $f s=() .47$ (Exp. \#119) and b) $f s=0.60($ Exp. $\# 121$ ) 
a)
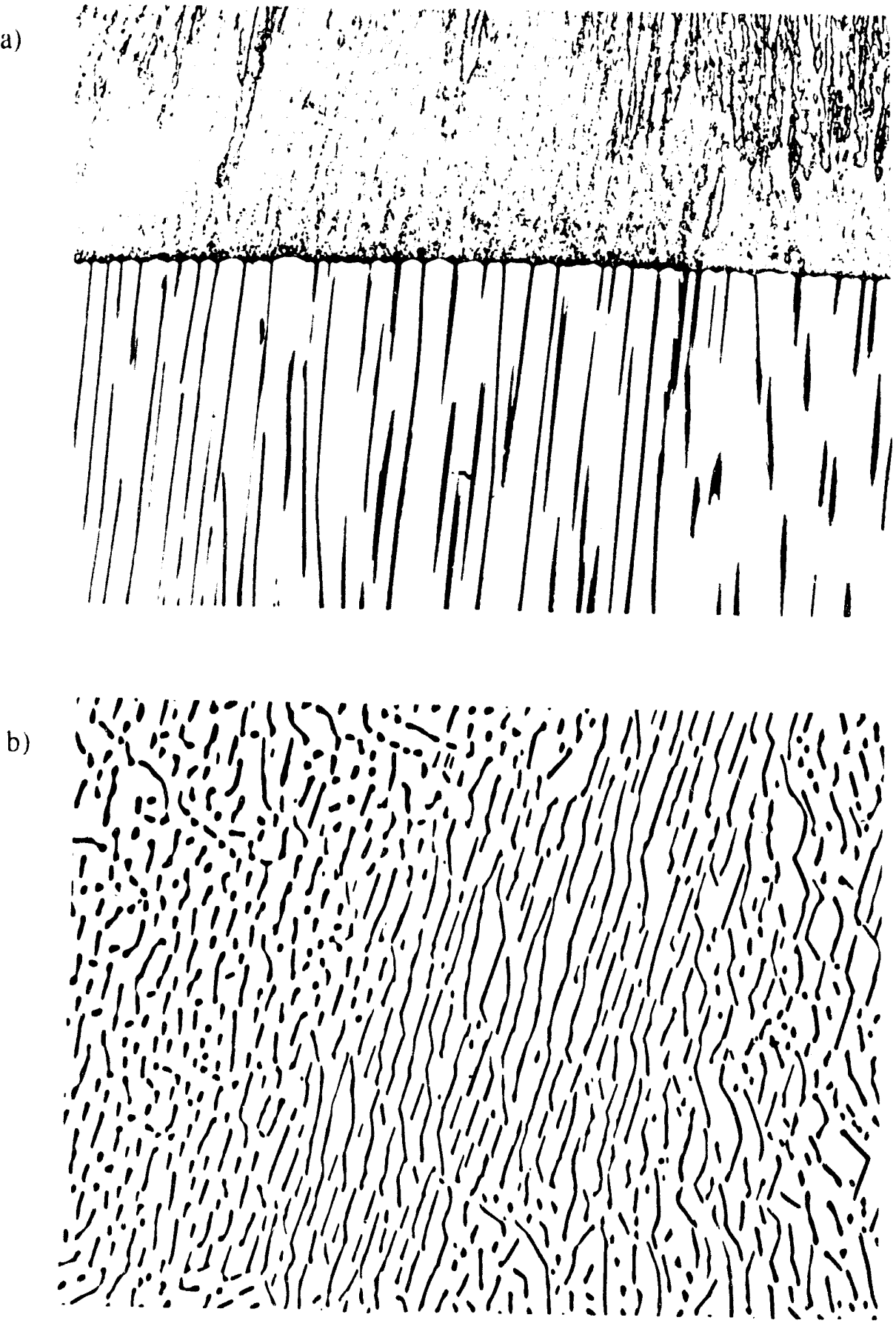

Figure 14. The eutectic structures in hypo eutectic alloys, 40x. a) Longitudinal and b) transverse views in Exp.\#106 (Al 23-0.8 $\mu \mathrm{m} / \mathrm{s}$, fs=0.87), 

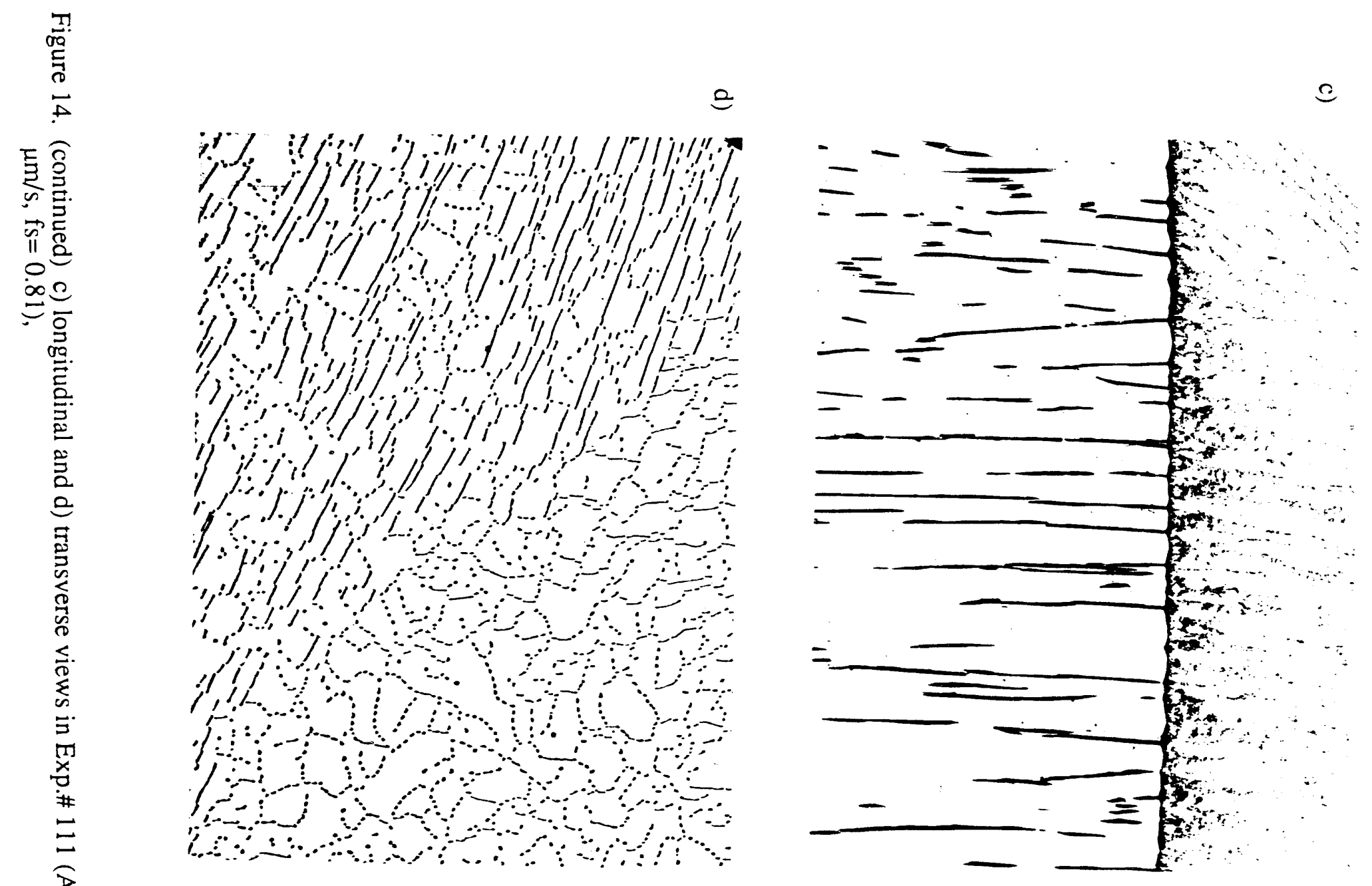
e)

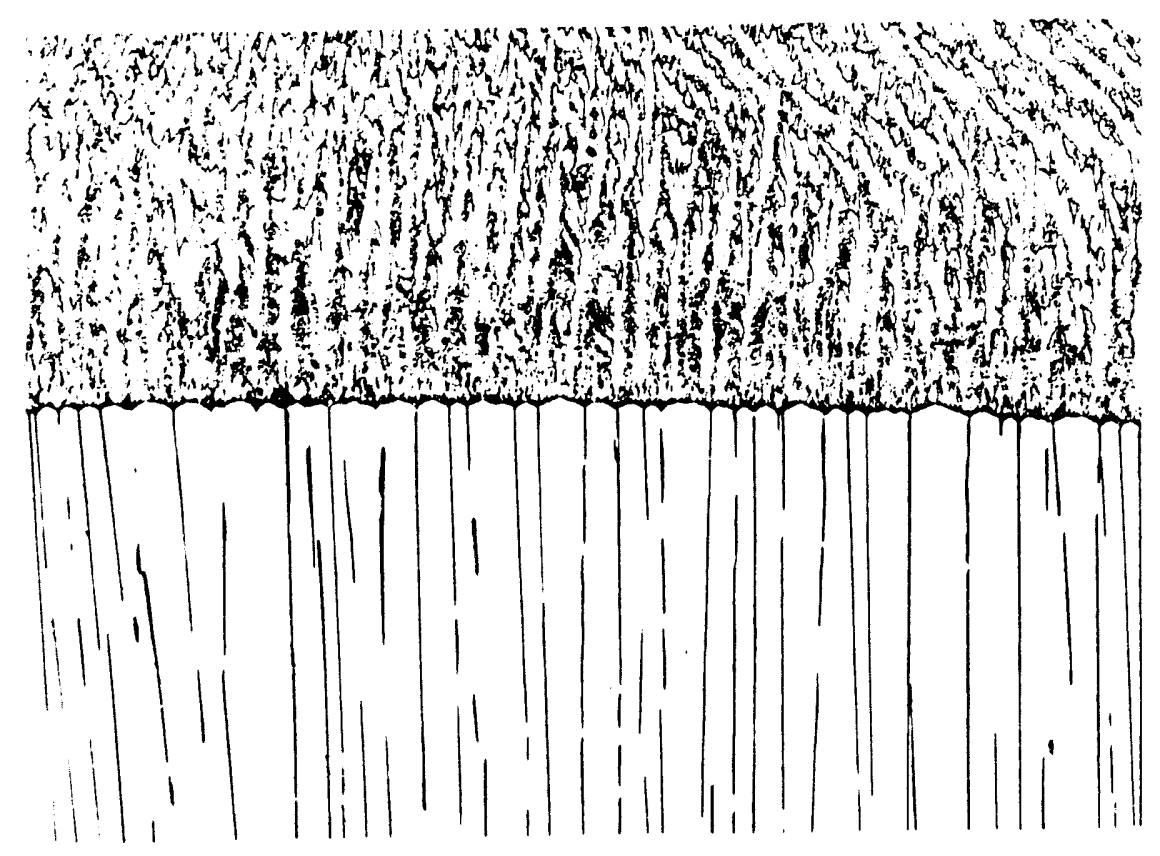

f)

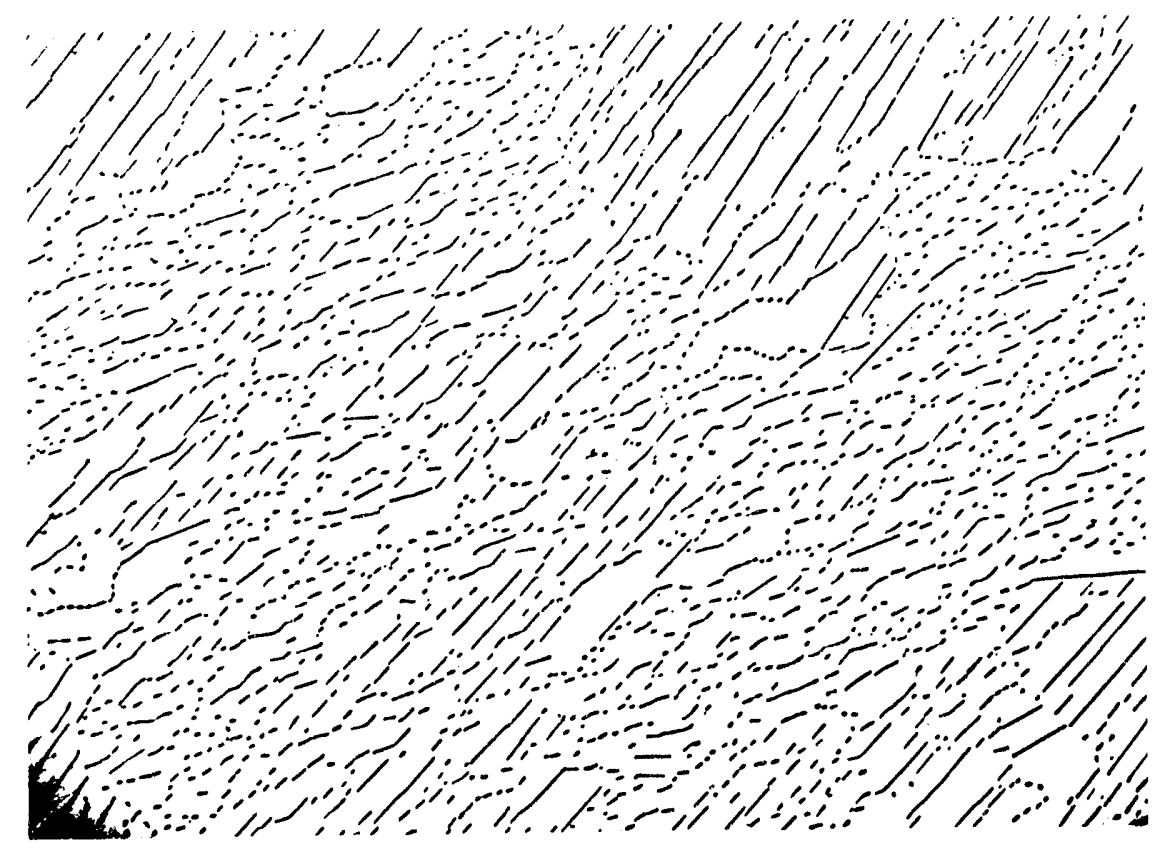

Figure 14. (continued) e) longitudinal and f) transverse views in Exp.\#105 (Al 24-3.2 $\mu \mathrm{m} / \mathrm{s}$, $f s=() .9)$ 
morphologies. When the closeness of the eutectic and peritectic compositions and temperatures are considered, this small composition variation seems a likely cause of the formation of different types of eutectics. The compositions of all different kinds of eutectics, which were found in hypo-eutectic alloys ( $\mathrm{Al} 23,23.5$ (I), 23.5(II), 24 alloys), eutectic composition alloys (Al 24.5(I), 24.5(II) alloys), and hyper-eutectic alloys (Al 25, 26 alloys), were analyzed by ICP (Induction Coupled Plasma) method, as shown in Table 5. The ICP method has a advantage to the wet chemical method by its small volume of sample analyzed. A piece of 50 $\mathrm{mg}$ size was required for an analyses for ICP, while $500 \mathrm{mg}$ was needed for a wet chemical analyses. A sample was cut $1 \mathrm{~mm}$ below the eutectic solid-liquid interface. Then, it was divided into two halves sections and both were analyzed to evaluate and increase the precision of this analysis. When the structures of center and edge were different, it was cut and analyzed at the center region and edge region. The compositions at the beginning of eutectic(position $\mathrm{A}$ in Table $5, \mathrm{fs}=0.85$ ) and the eutectic at the quenched solid-liquid interface (position $\mathrm{B}$, $\mathrm{fs}=0.98)$ were also compared in the sample of Exp.\# 85(Al 23-1.1 $\mu \mathrm{m} / \mathrm{s})$. The eutectic structure in sample A was almost changed to the $\gamma^{\prime}$ phase, but it could be distinguished by a layer of the $\beta$ phase which was formed at the beginning of the eutectic, similar to that shown in Figure 13(a)

The relation between the composition and eutectic morphology is shown in Figure 15, where the results of contaminated alloy, $\mathrm{Al} 23.5(\mathrm{I})$, were removed. The regular eutectics of 2P-R and 2P-L formed as off-eutectic planar interface above 24.9 at\% Al. The 2P-R(c) and 3P-R(c) eutectics were found as cellular type at 24.3-24.6 at\% Al and the 3P-L eutectic was found at 24.7 at $\%$ Al. These results indicate that the cellular type eutectics form at the lower side of the eutectic composition and regular types of rod and lamellar eutectics form at the higher side of the eutectic composition, where the transition occurs at the composition between 24.6 and 24.7 at $\%$ Al. The data also show that the 3-phase eutectic occurs only at compositions from 24.5 to 24.7 at $\% \mathrm{Al}$, whereas the 2-phase eutectics occur over the entire 
Table 5 Compositions at the different types of eutectics

\begin{tabular}{|c|c|c|c|c|c|c|c|c|}
\hline \multirow[t]{2}{*}{ Alloy } & \multirow{2}{*}{$\begin{array}{c}\text { Rate } \\
(\mu \mathrm{m} / \mathrm{s})\end{array}$} & \multirow[t]{2}{*}{ Exp.\# } & \multirow[t]{2}{*}{ fs } & \multirow[t]{2}{*}{ Position } & \multirow[t]{2}{*}{ Morphology } & \multicolumn{3}{|c|}{ Composition (Al at.\%) } \\
\hline & & & & & & Avg. & $\# 1$ & $\# 2$ \\
\hline \multirow[t]{4}{*}{23} & 0.8 & 106 & 0.85 & & $2 \mathrm{P}-\mathrm{L}+2 \mathrm{P} \cdot \mathrm{R}(\mathrm{cp})$ & 24.9 & 24.8 & 25.0 \\
\hline & 0.8 & 97 & 0.75 & & $2 \mathrm{P} \cdot \mathrm{L}+3 \mathrm{P}-\mathrm{R}(\mathrm{c})$ & 24.6 & 24.5 & 24.7 \\
\hline & 1.1 & 85 & 0.85 & $A(f s=0.85)$ &..${ }^{* \star}$ & 24.8 & 24.8 & + \\
\hline & & & 0.98 & $\mathrm{~B}(\mathrm{fs}=0.98)$ & $2 \mathrm{P}-\mathrm{R}(\mathrm{c})$ & 24.5 & 24.5 & + \\
\hline \multirow[t]{2}{*}{$23.5(\mathrm{I})^{\star}$} & 2 & 103 & 0.42 & $A(f s=0.42)$ & $3 \mathrm{P} \cdot \mathrm{L}^{++}$ & 24.3 & 24.3 & 24.3 \\
\hline & & & 0.91 & $B(f s=0.91)$ & $3 \mathrm{P} \cdot \mathrm{L}$ & 24.4 & 24.4 & 24.4 \\
\hline $23.5(\mathrm{II})$ & 3.2 & 111 & 0.81 & & $3 P-R(C)+2 P-R(c)$ & 24.5 & 24.4 & 24.5 \\
\hline 24 & 0.8 & 125 & 0.92 & & $2 P-R(c)$ & 24.6 & 24.5 & 24.7 \\
\hline \multirow[t]{4}{*}{$24.5(\mathrm{I})$} & 5 & 61 & 0.42 & Center & $3 P \cdot R(c)$ & 24.5 & 24.6 & 24.4 \\
\hline & & & & Edge & $2 \mathrm{P}-\mathrm{R}(\mathrm{c})$ & 24.3 & 24.5 & 24.1 \\
\hline & 2.5 & 58 & 0.40 & & $\gamma^{\prime}+3 P \cdot L$ & 24.4 & 24.5 & 24.2 \\
\hline & 61 & 129 & 0.65 & & $\gamma^{\prime}+3 P-L$ & 24.6 & 24.6 & 24.5 \\
\hline \multirow[t]{6}{*}{$24.5(\mathrm{II})$} & 0.8 & 117 & 0.40 & & $3 \mathrm{P} \cdot \mathrm{L}$ & 24.7 & 24.6 & 24.8 \\
\hline & 3.2 & 118 & 0.47 & Center & 3P-L & 24.7 & 24.7 & 24.7 \\
\hline & & & & Edge & $3 P-R(c)$ & 24.6 & 24.6 & 24.6 \\
\hline & 5 & 110 & 0.34 & Center & $3 P-R(c)+3 P-L$ & 24.7 & 24.7 & 24.7 \\
\hline & & & & Edge & $2 \mathrm{P} \cdot \mathrm{R}(\mathrm{c})$ & 24.5 & 24.5 & 24.5 \\
\hline & 61 & 128 & 0.65 & & 3P-L & 24.7 & 24.6 & 24.8 \\
\hline \multirow[t]{3}{*}{2.5} & 0.8 & 4 & 0.30 & & 2P-R(cp) & 25.5 & 25.5 & 25.5 \\
\hline & 3.2 & 19 & 0.44 & & $2 P \cdot R(c p)+2 P \cdot L$ & 25.2 & 25.1 & 25.3 \\
\hline & 6.3 & 3 & 0.74 & & $2 \mathrm{P}-\mathrm{L}+2 \mathrm{P}-\mathrm{R}(\mathrm{cp})$ & 25.0 & 24.8 & 25.1 \\
\hline 26 & 0.8 & 38 & 0.32 & & $2 \mathrm{P}-\mathrm{L}$ & 26.8 & 26.8 & 26.7 \\
\hline
\end{tabular}

+ It was anayzed one time.

++ Eutectic had transformed to $\gamma^{\prime}$, so this is assumed structure.

* This alloy was contaminated by $\mathrm{Fe}, \mathrm{Cr}, \mathrm{Mn}, \mathrm{Si}$, and $\mathrm{Nb}$.

** Eutectic had transformed to all most $\gamma^{\prime}$ (small amount of untransformed $\beta$ ), so it is unknown. 


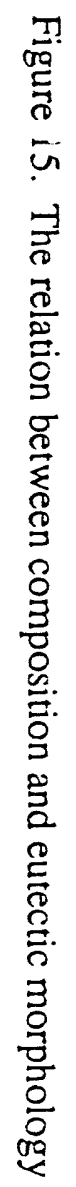

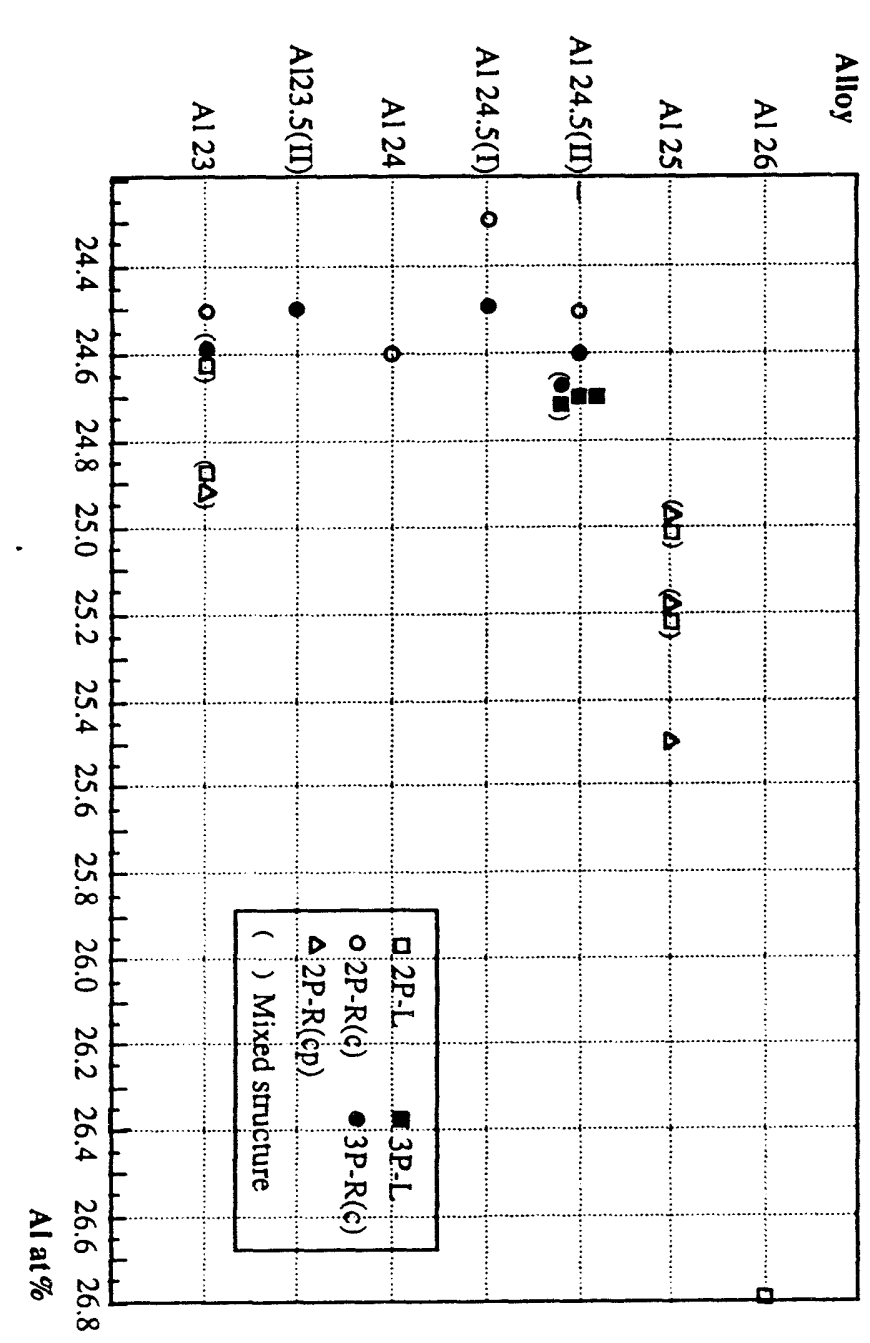


composition range of 24.3 to 26.8 at $\% \mathrm{Al}$.

It was also found that the eutectic formed after the $\gamma^{\prime}$ planar at low rates in hypoeutectic compositions $(23,23.5$ (II), 24, 24.5(I)). The beginning of the eutectic might be formed with hyper-eutectic compositions by undercooling of the $\gamma^{\prime}$ planar interface. This idea is supported by the result that the 3P-L eutectic starts to grow from the $\beta$ layer which is formed at the end of the $\gamma^{\prime}$ planar at low rates, as seen in Figure 13(a). Then, this 3P-L eutectic changes to the 3P-R(c) eutectic which forms at compositions on the lower side of the eutectic composition, when fs increases from 0.47 to 0.60 as seen in Figure 13(b). Note also that Part

III showed that the eutectic was formed from $\beta$ layer formation in front of the $\gamma^{\prime}$ lower band upon quenching. There is good agreement for the formation of the $\beta$ layer after the growth of the $\gamma^{\prime}$ planar here, as the first step in the nucleation of the 3P-L eutectic.

The growth front temperature of the $\gamma^{\prime}$ planar is though to be controlled by the G/V value. The most high G/V condition of Exp.\#106 (Al 23-0.8 $\mu \mathrm{m} / \mathrm{s})$ is expected to reduce the growth front temperature the most before nucleating the $\beta$ phase with the eutectic because the composition of the eutectic was as high as 24.9 at\% Al. The peritectic and eutectic temperatures and compositions are so close that the $G / V$ value is an important factor for the formation of a meta-stable phase. More precise experiments to control G/V values are required and these experiments are under way.

\section{Fast Quench Experiments}

Three phases are clearly formed at the growth front as shown in Figure 4. However, a strong question was raised whether the three phase eutectic could be formed in the binary system. In order for a three phase eutectic to form, the diffusion in front of solid-liquid interface requires the liquid/ $\gamma$ concentration of $\mathrm{Al}\left(\mathrm{C}_{2}\right.$ in Figure 16$)$ to be higher than the liquid/ $\gamma\left(C_{3}\right)$ concentration. However, $C_{2}$ is lower than $C_{3}$ as is seen on the phase diagram of Figure 9. From a simple diffusion requirement three phase coupled growth is unlikely to form. 


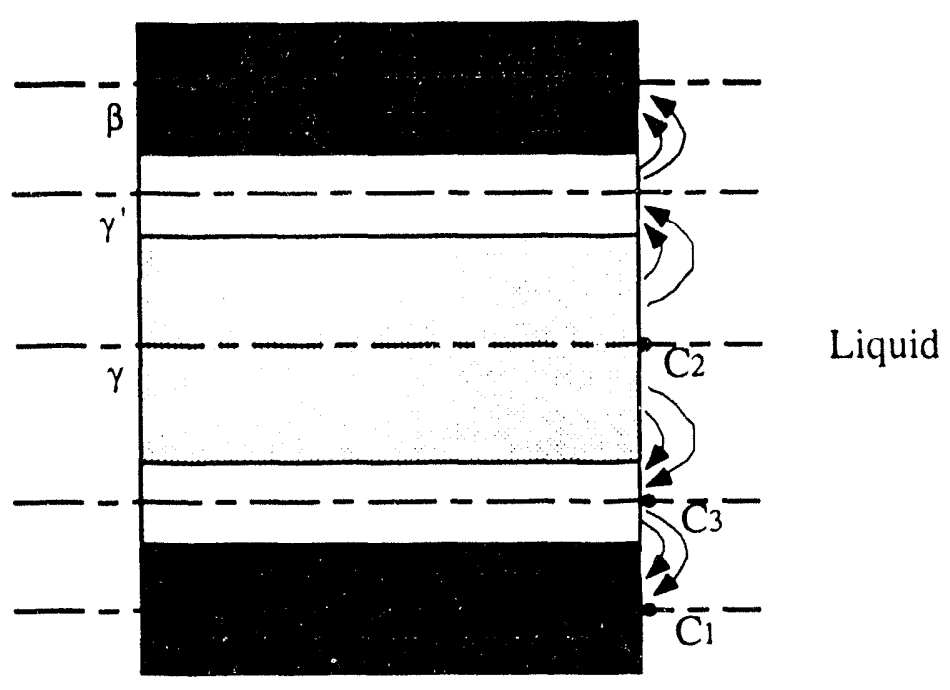

Figure 16. Schematic diagram of the possible solute distribution in liquid for the 3-phase eutectic 
When the fast solid-state transformation is considered in this system, the 3P-L eutectic might be the $\gamma+\beta$ or $\gamma^{\prime}+\beta$ eutectic. In order to reduce the solid state transformation during the quench procedure the Ga-In liquid was used as a quench media. The 3P-L eutectics were

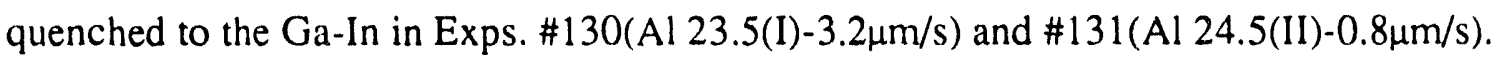

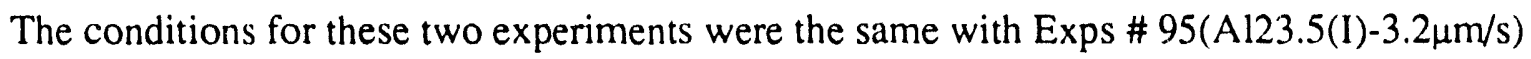
and $126(\mathrm{Al} 24.5(\mathrm{II})-0.8 \mu \mathrm{m} / \mathrm{s})$ of the water quench, which had the $100 \% 3 \mathrm{P}-\mathrm{L}$ eutectics.

The eutectic structures of Exp. \# 131 and Exp. \# 126(Ga-In quenches) were identical to those of Exp.\#130 and Exp.\# 95(water quenches) respectively. However, the thicknesses of $\gamma$ phase between the $\beta$ and $\gamma$ phases were reduced at the solid-liquid interface in the fast quench of Ga-In, as shown in Figure 17(compare with Figure 4(a)). These results indicate that the formation of $\gamma^{\prime}$ layer very depends on the quench rates. In order to obtain more fast quench a smaller diameter of alumina tube $(3 \mathrm{~mm})$ was used while the diameter of usual tube was $5 \mathrm{~mm}$. If one assumes that the thermal conductivities of the alumina tube and the sample are the same, the cooling rate is inversely proportional to the radius of the rod. Then,the quench rate is expected to be increased 5/3 times in the $3 \mathrm{~mm}$ I.D.tube. However, the cooling rate must be higher than $5 / 3$ times in the $3 \mathrm{~mm}$ tube because the thermal conductivity of alumina tube is less than that of $\mathrm{Ni}-\mathrm{Al}$ sample and the wall thickness of the $3 \mathrm{~mm}$ I.D. alumina tube is $1 \mathrm{~mm}$ and that of $5 \mathrm{~mm}$ tube is $1.5 \mathrm{~mm}$. The $\gamma^{\prime}$ layers were reduced in this fastest quench $(3$ $\mathrm{mm}$ diameter in the Ga-In) and this result is shown in Figure 18.

Table 6 shows how the thicknesses of the $\gamma, \gamma^{\prime}$, and $\beta$ phases at the solid-liquid interface are changed according to the quench rates. In order to overcome the variations of the thicknesses resulting from the angle of the sections, transverse sections were cut $0.5 \mathrm{~mm}$ below the solid-liquid interface of Figure 17 and the average spacing was measured on this transverse section. The spacings were quite constant on the transverse sections, where those were measured perpendicular to each phase. The rotated angle at the solid-liquid interface in the longitudinal sections could be deduced from the average spacing on the transverse section. 
a)

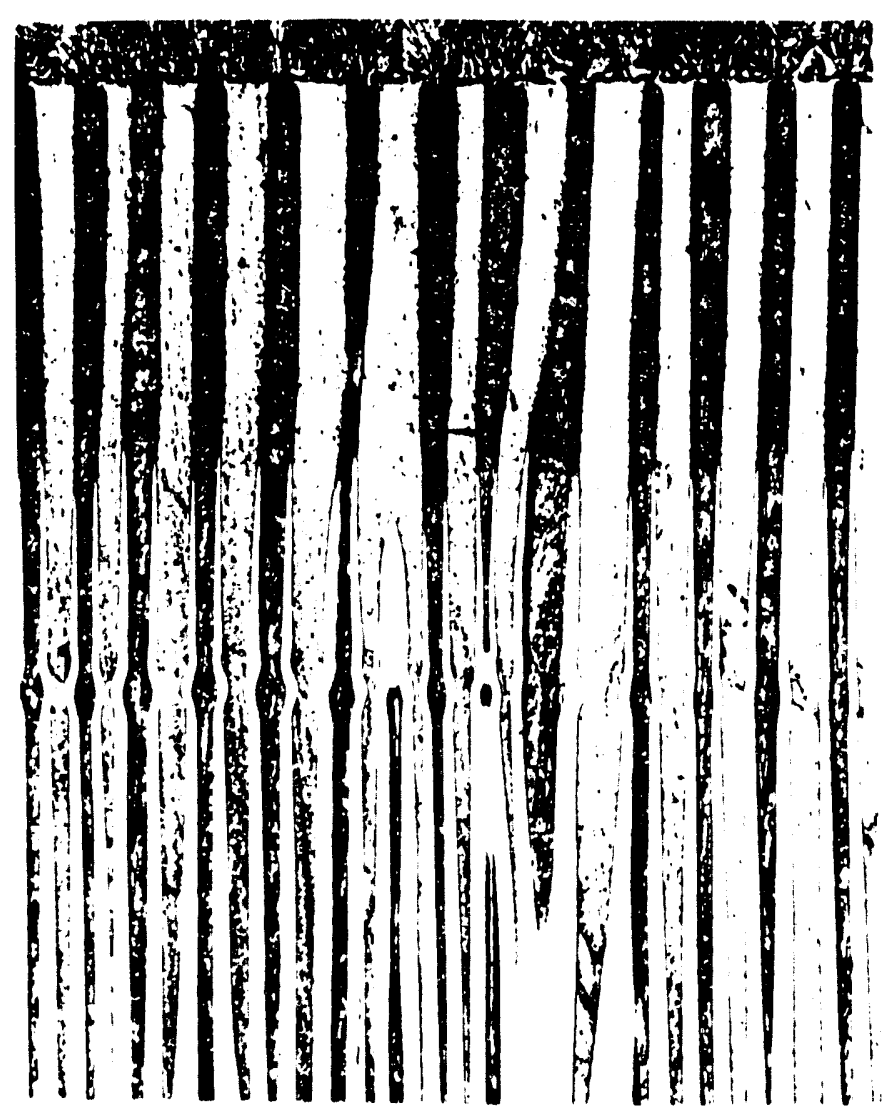

Figure 18. The quenched solid-liquid interface in Exp. 134(Al $24.5-0.8 \mathrm{~mm} / \mathrm{s})$ where it was queched into the Ga-In and the $3 \mathrm{~mm}$ alumina tube was used for a container. This quench was the fastest quench in this study. a) $100 \mathrm{x}$ 
b)

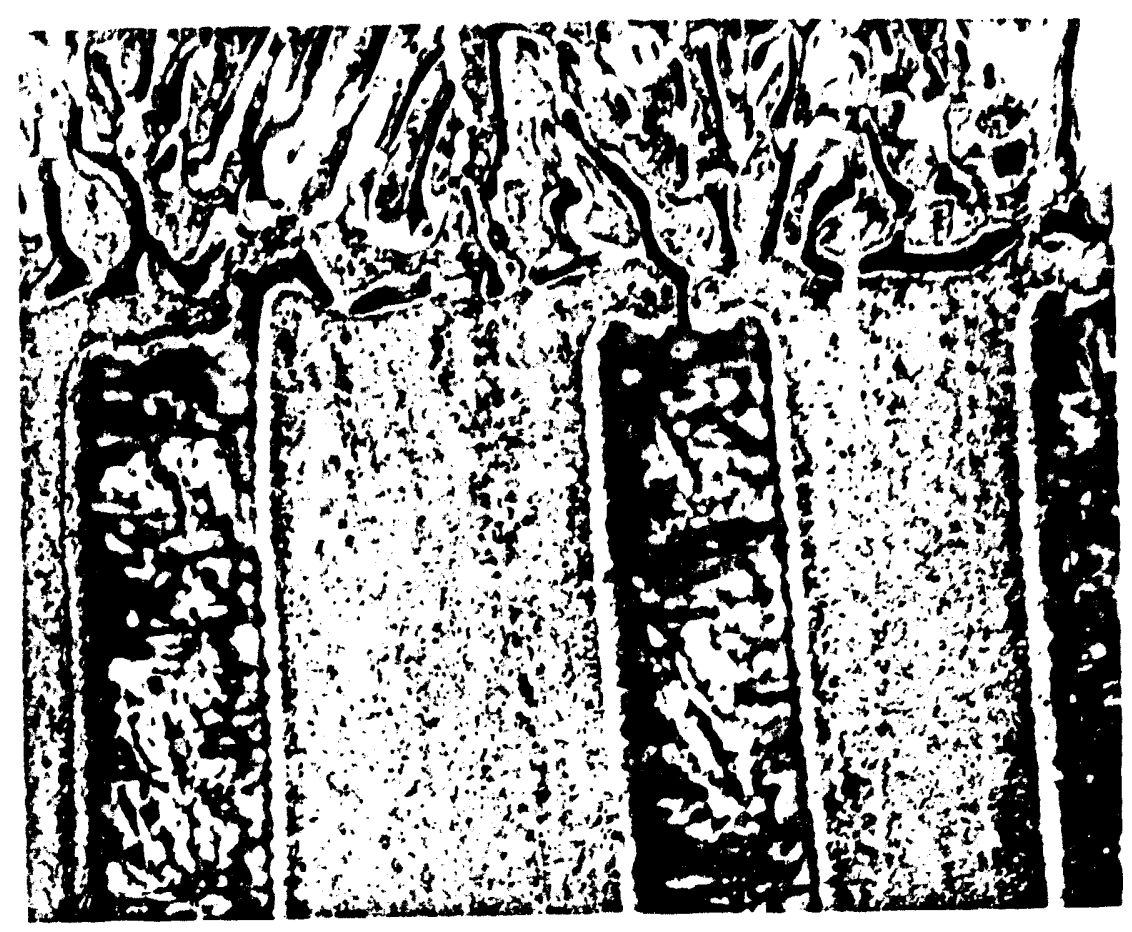

Figure 18. (continued) and b) $1000 x$ 
Table 6. Variations in the thickness of each phase in the eutectic structure at the solid-liquid interface according to the quench rates in some experiments with 3P-L morphology

\begin{tabular}{|c|c|c|c|c|c|c|c|}
\hline Alloy & $\mathrm{V}(\mu \mathrm{m} / \mathrm{s})$ & \multirow{2}{*}{ Exp.\# } & \multirow{2}{*}{ Quench } & \multicolumn{4}{|c|}{ Thickness $(\mu \mathrm{m})$} \\
\cline { 3 - 8 } & & & & $\delta_{\gamma}$ & $\delta_{\beta}$ & $\delta_{\gamma^{\prime}}$ & $\lambda_{(\beta-\beta)}$ \\
\hline \hline \multirow{3}{*}{ Al 23.5(I) } & \multirow{2}{*}{0.8} & 99 & Water & 19 & 8 & 4 & 35 \\
\cline { 3 - 8 } & & 133 & Ga-In* & 21 & 12 & 1 & 35 \\
\cline { 3 - 8 } & \multirow{2}{*}{3.2} & 92 & Water & 14 & 2 & 2 & 20 \\
\cline { 3 - 8 } & & 130 & Ga-In & 14 & 3 & 1 & 20 \\
\hline \multirow{2}{*}{ Al 24.5(II) } & \multirow{2}{*}{0.8} & $115(117)$ & Water & 17 & 9 & 4 & 36 \\
\cline { 3 - 8 } & & 131 & Ga-In & 18 & 13 & 2 & 36 \\
\cline { 3 - 8 } & & 134 & Ga-In* & 20 & 16 & 1 & 38 \\
\hline
\end{tabular}

* These experiments were done in the $3 \mathrm{~mm}$ diameter alumina tube to increase the quench rate. Usual diameter of the tube was $5 \mathrm{~mm}$.

The thicknesses of each phase at the solid-liquid interface in the longitudinal sections were corrected by this rotated angle. Thickness of $\gamma^{\prime}$ was increased while those of $\beta$ and $\gamma$ were reduced at the relative slow quench rate of water, where the $\beta$ phase was reduced more than the $\gamma$ phase. This result indicates that the $\gamma^{\prime}$ phase might be formed from the $\beta$ phase more than the $\gamma$ phase upon quenching. As will be shown in discussion section, these fast quench experiments provide strong evidence that the 3P-L eutectic is the $\gamma+\beta$ eutectic and the $\gamma^{\prime}$ layer between the $\gamma$ and $\beta$ phases at the solid- liquid interface forms during the quench procedure.

\section{Cellular Type Eutectic}

The cellular type eutectic is an unusual morphology in the eutectic system. Figure 15 shows that the cellular type forms on the lower side of eutectic compositions where the composition is close to the $\gamma^{\prime}$ phase. This cellular eutectic structure consists of pure $\gamma^{\prime}$ cells having either $\beta$ phase rods in the cell boundaries as in Figure 12, or both $\beta$ phase rods and $\gamma$ phase sheets in the cell boundaries, as in Figure 8. The former is coded as 2P-R(c) and the latter $3 \mathrm{P}-\mathrm{R}(\mathrm{c})$. 
Figure 19(b) shows the $\gamma^{\prime}$ cells in the eutectic cellular structure at $10 \mu \mathrm{m} / \mathrm{s}$ changing to the separated $\gamma^{\prime}$ cells (white single phase regions) at $25 \mu \mathrm{m} / \mathrm{s}$ as seen in Fig.19(d). The $\gamma^{\prime}$ cells form as a large separated cell and appears to grow with the eutectic at almost the same temperature at $25 \mu \mathrm{m} / \mathrm{s}$ in the 24.5(I) alloy, as shown in Figure 20. It appears that the $\gamma^{\prime}$ cell regions of the cellular eutectic evolve into large single phase cells as the rate increases and the interface becomes more unstable to constitutional supercooling.

The 3P-R(c) is a really strange structure to form 3-phases at the growth front while the 3P-L eutectic is thought to be the $\gamma+\beta$ metastable eutectic which is really a $2 \mathrm{P}-\mathrm{L}$ structure at the growth front. The 3P-R(c) structure looks like the mixed structure of the $\gamma+\beta$ eutectic with the $\gamma^{\prime}+\gamma$ coupled peritectic. The $\gamma+\gamma^{\prime}$ coupled peritectic structure is shown in Figure 21. This coupled peritectic was found as a transition structure between the $\gamma$ planar to the $\gamma$ planar at low rates in hypo-eutectic alloys. The current study showed that the coupled peritectic growth depends on the $G / V$ value. The coupled $\gamma+\gamma^{\prime}$ structure formed at certain range of $G / V(0.78$ $\left.1.28 \times 10^{5}{ }^{\circ} \mathrm{Cs} / \mathrm{cm}^{2}\right)$ whereas the band structure of $\gamma+\gamma^{\prime}$ formed at high $\mathrm{G} / \mathrm{V}$ values $(3.76-5.18$ $\mathrm{x} 10^{5}{ }^{\circ} \mathrm{Cs} / \mathrm{cm}^{2}$ ). The coupled and band structures formed together at the medium $\mathrm{G} / \mathrm{V}$ values between those two conditions $\left(1.93-3.55 \times 10^{5}{ }^{\circ} \mathrm{Cs} / \mathrm{cm}^{2}\right)$. A big question arose how the $\gamma+\gamma^{\prime}$ coupled peritectic forms with the $\gamma^{\prime}+\beta$ eutectic at the same growth front. The $3 \mathrm{P}-\mathrm{R}(\mathrm{c})$ eutectic might also depend on the G/V values if it is really a mixed structure of $\gamma+\gamma^{\prime}$ coupled peritectic and $\gamma^{\prime}+\beta$ eutectic. In order to understand the 3P-R(c) eutectic the study for the coupled peritectic should be continued. This study is currently underway.

\section{Eutectic Spacing}

The eutectic spacings in the various types of morphologies were estimated according to the solidification rates as shown in Table 7. The spacings were measured in the transverse sections $1 \mathrm{~mm}$ below the solid-liquid interface. These values are the average values at different morphologies and rates. The $2 \mathrm{P}-\mathrm{L}, 2 \mathrm{P}-\mathrm{R}(\mathrm{cp})$, and $3 \mathrm{P}-\mathrm{L}(=\gamma+\beta$ lamellar) eutectisis are regular 
a)

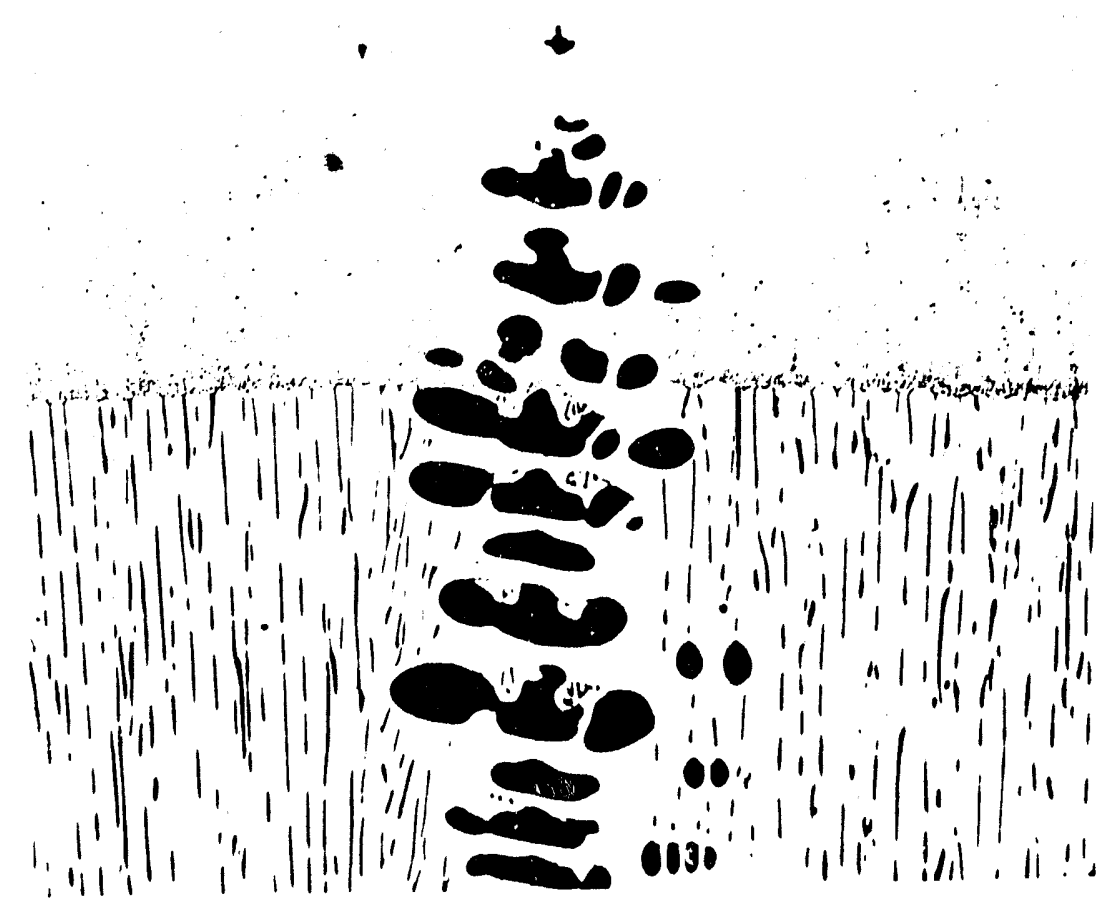

b)

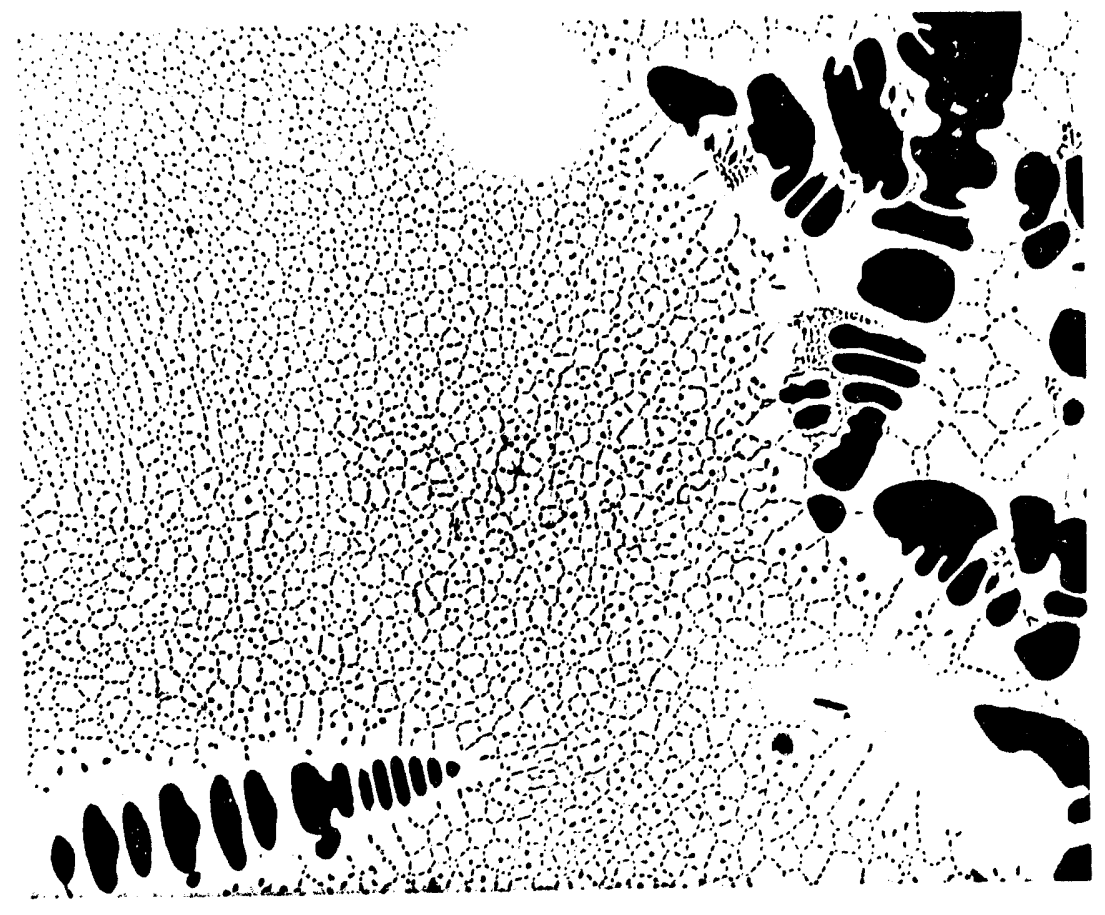

Figure 19. The cellular type eutectics in the $\beta$ interdendrites, 50x. a) Longitudinal and b) transeverse views of the $2 \mathrm{P}-\mathrm{R}(\mathrm{c})$ eutectic in Exp.\# $53(10 \mu \mathrm{m} / \mathrm{s})$, 


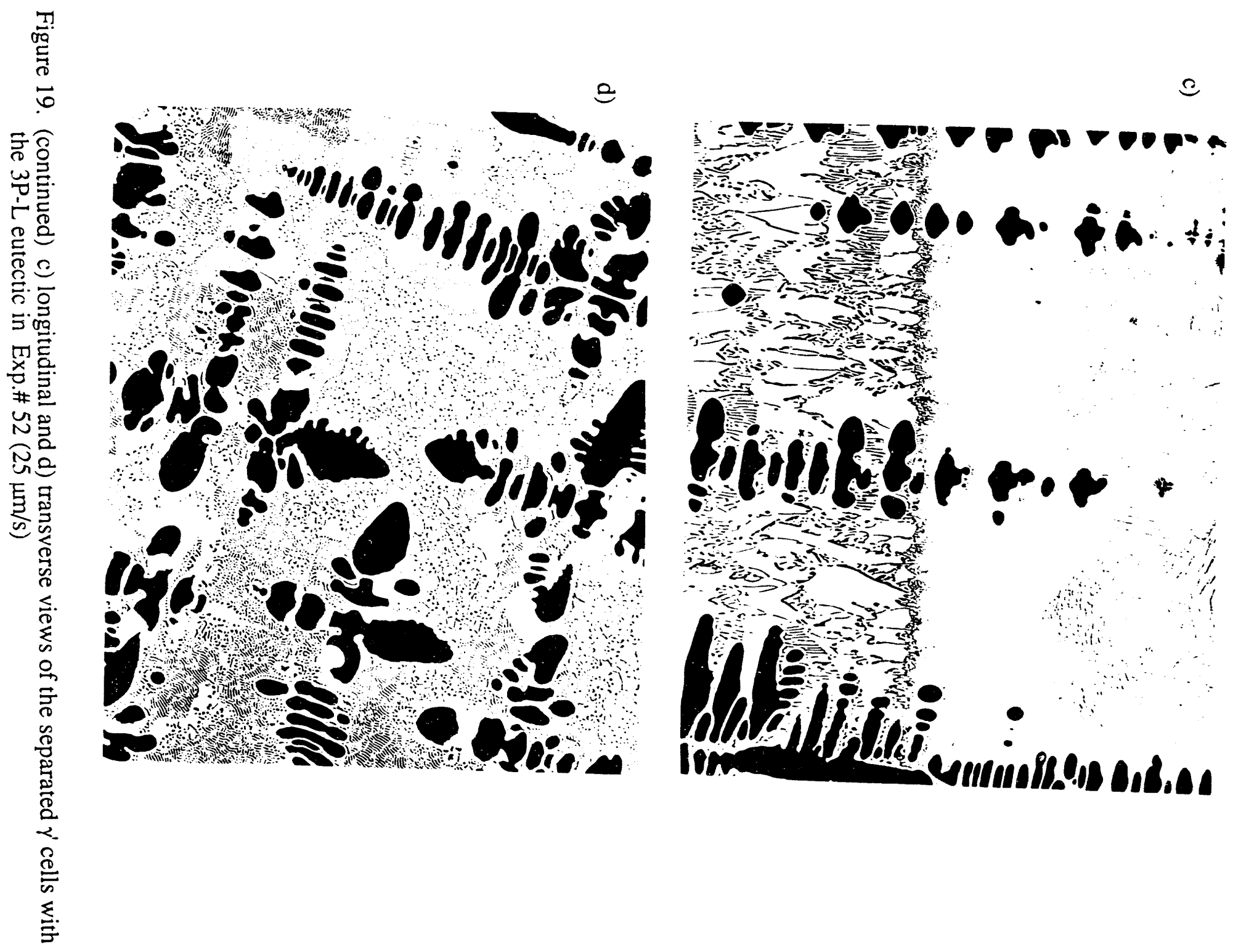



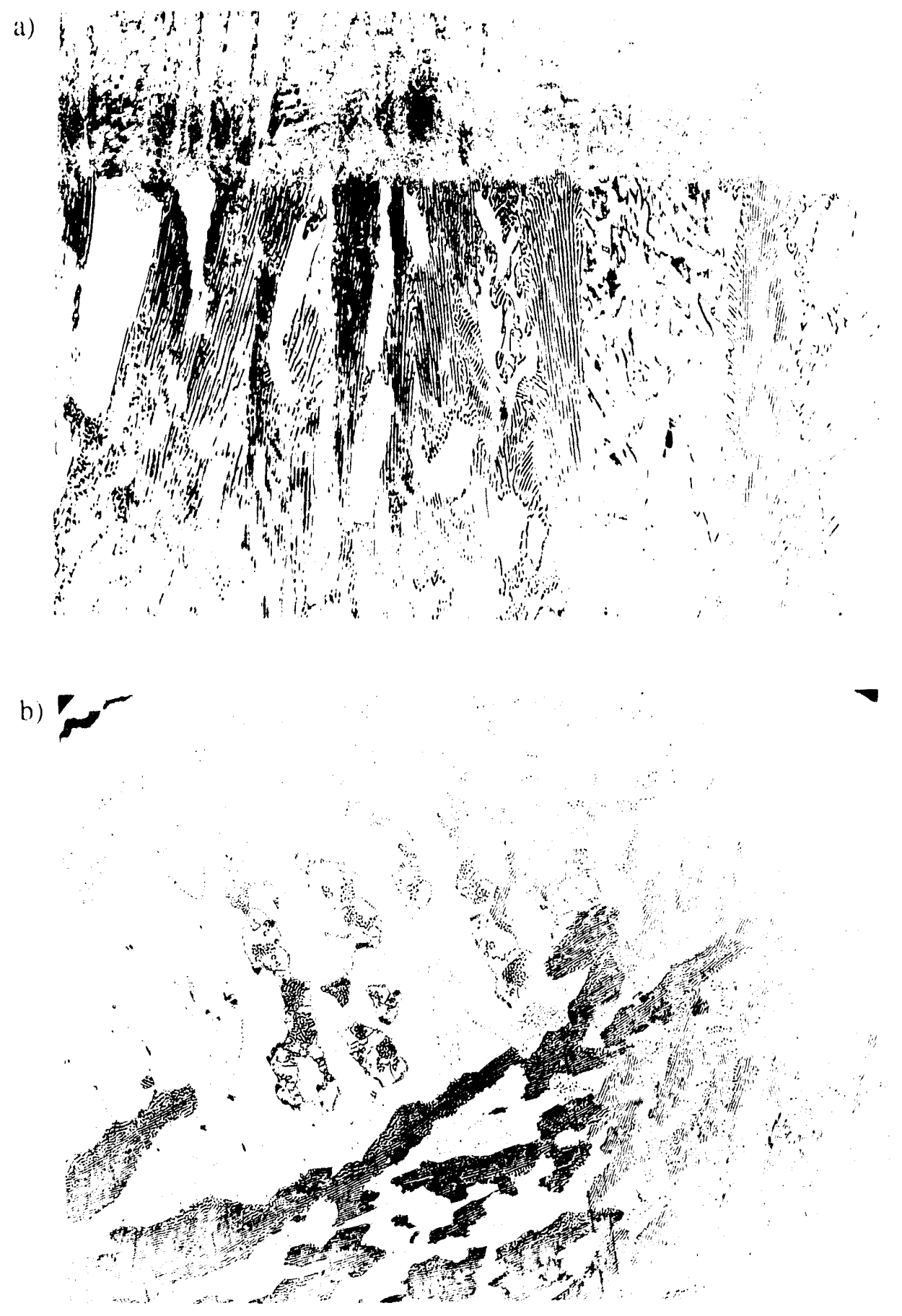

Figure 20. Optical micrographs at $25 \mu \mathrm{m} / \mathrm{s}$ in $\mathrm{Al} 24.5$ (I) alloy (Exp.\# .58). a) Longitudinal and $b$ ) transverse views, $40 x$ 


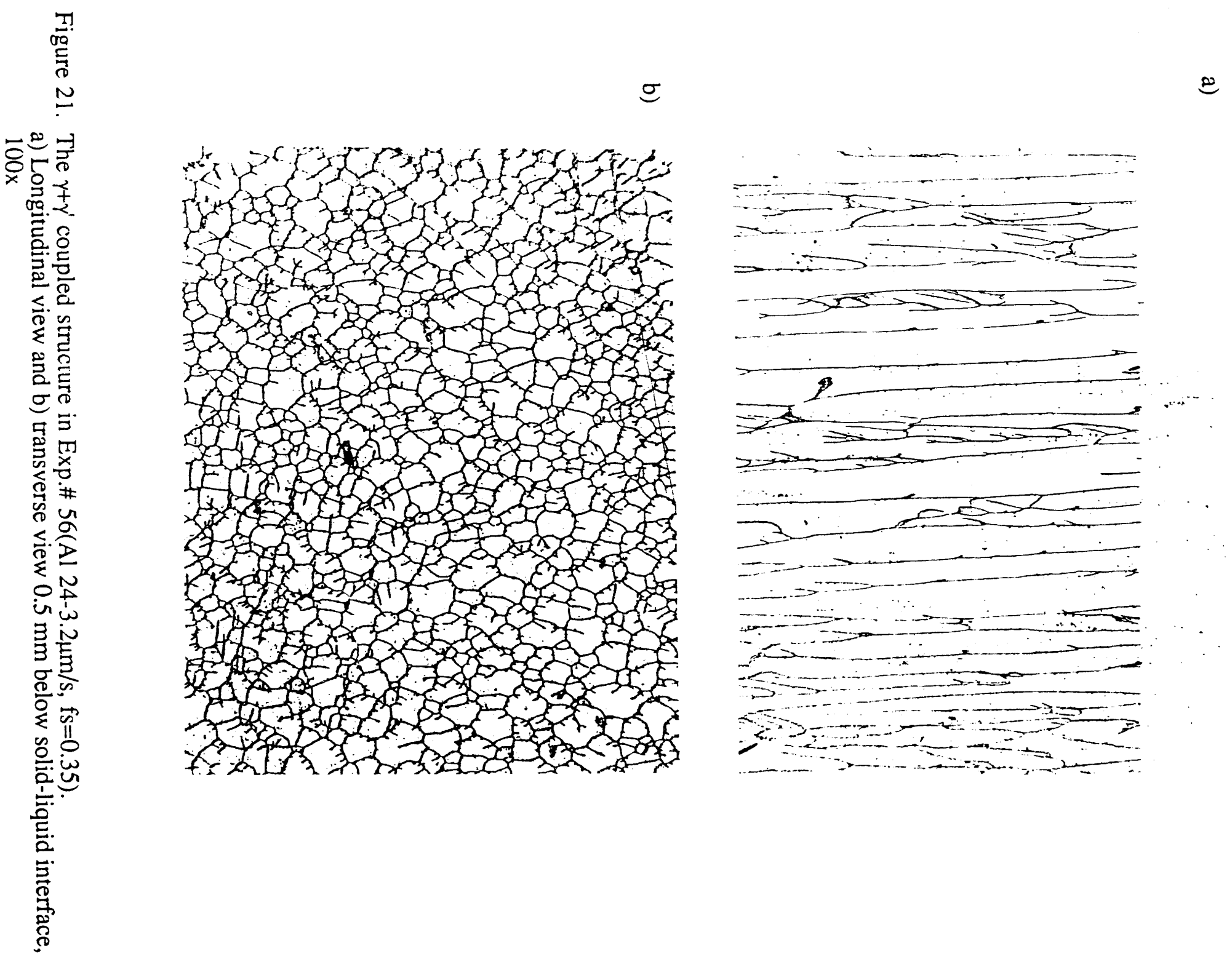


Table 7. The eutectic spacings in the various eutectic structures according to the soldification rates

\begin{tabular}{|c|c|c|c|c|}
\hline Rate $(\mu \mathrm{m} / \mathrm{s})$ & Phases & Type & Exp.\# & Avg. $\lambda(\mu \mathrm{m})$ \\
\hline \multirow[t]{4}{*}{$\overline{0.8}$} & \multirow[t]{3}{*}{$\overline{\gamma^{\prime}+\beta}$} & $\overline{2 \mathrm{P}-\mathrm{L}}$ & $38,97,109$ & $\overline{61.8}$ \\
\hline & & $2 \mathrm{P}-\mathrm{R}(\mathrm{cp})$ & 4,106 & 63.1 \\
\hline & & $2 \mathrm{P}-\mathrm{R}(\mathrm{c})$ & 121,125 & 53.5 \\
\hline & $\gamma+\beta$ & $3 \mathrm{P}-\mathrm{L}$ & $115,99,107$ & 35.7 \\
\hline$\overline{2}$ & $\overline{\gamma^{\prime}+\beta}$ & $3 \mathrm{P}-\mathrm{L}$ & 100,103 & 24.6 \\
\hline \multirow[t]{4}{*}{$\overline{3.2}$} & \multirow[t]{3}{*}{$\gamma^{\prime}+\beta$} & $2 \mathrm{P}-\mathrm{L}$ & $24,32,19,105,111$ & 33.4 \\
\hline & & $2 \mathrm{P}-\mathrm{R}(\mathrm{cp})$ & 24,32 & 32.1 \\
\hline & & $2 \mathrm{P}-\mathrm{R}(\mathrm{c})$ & $59,98,105,111$ & 30.7 \\
\hline & $\gamma+\beta$ & $3 \mathrm{P}-\mathrm{L}$ & $118,92,95,102$ & 20.0 \\
\hline \multirow[t]{4}{*}{5} & \multirow{3}{*}{$\frac{1}{\gamma^{\prime}+\beta}$} & $2 \mathrm{P}-\mathrm{L}$ & 54 & 26.0 \\
\hline & & $2 \mathrm{P}-\mathrm{R}(\mathrm{cp})$ & 54 & 26.5 \\
\hline & & $2 \mathrm{P}-\mathrm{R}(\mathrm{c})$ & $54,61,62,110$ & 24.4 \\
\hline & $\gamma+\beta$ & 3P-L & 110 & 14.5 \\
\hline \multirow[t]{2}{*}{6.3} & \multirow{2}{*}{$\frac{1}{\gamma^{\prime}+\beta}$} & $2 \mathrm{P}-\mathrm{L}$ & 3 & 22.0 \\
\hline & & $2 \mathrm{P}-\mathrm{R}(\mathrm{cp})$ & 3 & 22.5 \\
\hline \multirow[t]{2}{*}{ 10) } & $\overline{\gamma^{\prime}+\beta}$ & $2 \mathrm{P}-\mathrm{R}(\mathrm{c})$ & 53,110 & 18.2 \\
\hline & $\gamma+\beta$ & $3 \mathrm{P}-\mathrm{L}$ & 110 & 10.8 \\
\hline \multirow[t]{2}{*}{25} & $\overline{\gamma^{\prime}+\beta}$ & $2 P-R(c)$ & 23,52 & 14.5 \\
\hline & $\gamma+\beta$ & $3 \mathrm{P}-\mathrm{L}$ & $23,52,58$ & 7.9 \\
\hline 61 & $\overline{\gamma+\beta}$ & $3 \mathrm{P}-\mathrm{L}$ & $\begin{array}{l}8,10,18,25,29 \\
128,129\end{array}$ & $\overline{6.4}$ \\
\hline
\end{tabular}

morphlogies in the eutectic, but the cellular types of $2 \mathrm{P}-\mathrm{R}(\mathrm{c})$ and $3 \mathrm{P}-\mathrm{R}(\mathrm{c})$ are unusual. There is not so much difference in the spacings between the lamellar and rod types (almost the same) in the $\gamma^{\prime}+\beta$ eutectic. In the 2P-R(c) morphology one can consider the cylindrical boundaries of the $\gamma^{\prime}$ cells as containing a rod type $\gamma^{\prime}+\beta$ eutectic around the $\gamma^{\prime}$ cells. The spacings of the $\gamma^{\prime}+\beta$ eutectic of the cellular type (2P-R(c)) was measured around the $\gamma^{\prime}$ cell and these results agreed with the spacings of the regular rod eutectic (2P-R(cp)). The spacings of the 3P-R(c) eutectic were so irregular that those were not measured because the spacings between the $\beta$ phases were disturbed by the $\gamma$ phases. The relations between the eutectic spacings of the equilibrium $\gamma^{\prime}+\beta$ eutectic and meta-stable $\gamma+\beta$ eutectic solidification rates are shown in Figure 22.

It is well known that the eutectic spacings are inversely proportional to square root of the velocity and the value of $\lambda^{2} \mathrm{~V}$ is constant [16], 


$$
\begin{aligned}
& \lambda^{2} \mathrm{~V}= \phi^{2} \mathrm{~K}_{2} / \mathrm{K}_{1}=\text { constant } \\
& \mathrm{K}_{1}=\frac{\bar{m} C_{0}}{D} \frac{P}{f_{\alpha} f_{\beta}} \\
& \mathrm{K}_{2}=2 \bar{m}\left(\frac{\Gamma_{\alpha} \sin \theta_{\alpha}}{\left|m_{\alpha}\right| f_{\alpha}} \frac{\Gamma_{\beta} \sin \theta_{\beta}}{m_{\beta} f_{\beta}}\right)
\end{aligned}
$$

where $\bar{m}=\left|m_{\alpha}\right| m_{\beta} /\left(\left|m_{\alpha}\right|+m_{\beta}\right), \mathrm{m}_{\alpha}$ and $\mathrm{m}_{\beta}$ are liquidus slopes, $\mathrm{C}_{0}=\mathrm{C}_{\beta}-\mathrm{C}_{\alpha}, \mathrm{P}$ is the function of volume fraction $\left(\approx\left[1-0.205 \exp \left(-24 \mathrm{f}_{\alpha} \mathrm{f}_{\beta}\right)\right] \times 0.3251\left(\mathrm{f}_{\alpha} \mathrm{f}_{\beta}\right)^{1.63}\right), \mathrm{f}_{\alpha}$ and $\mathrm{f}_{\beta}$ are volume fractions of $\alpha$ and $\beta$ phases, $\theta_{\alpha}$ and $\theta_{\beta}$ are contact angles at the theree phase junction, $\Gamma_{\alpha}$ and $\Gamma_{\beta}$ are GibbsThomson coefficients ( $=$ ratio of the surface energy to the entrophy, $\gamma_{\alpha \mathrm{L}} / \Delta \mathrm{S}_{\mathrm{f}}$ and $\gamma_{\beta \mathrm{L}} / \Delta \mathrm{S}_{\mathrm{f}}$ ), and $\phi$ is the ratio of actual to extreme spacing which is 1 in the normal eutectic. Figure 22 shows that the experimental results agree with this relationship, as the slopes are -0.5 in the $\gamma^{\prime}+\beta$ equilibrium eutectic and -0.42 in the meta-stable $\gamma+\beta$ eutectic. These results agree with equation (1). Also, the theoretical values of $\lambda^{2} \mathrm{~V}$ by the equation (1) could be compared with the experimental values of Table 8 in the lamellar type of $\gamma^{\prime}+\beta$ and $\gamma+\beta$ eutectics. When the $\Gamma$ and $\theta$ values of $\gamma, \gamma^{\prime}$, and $\beta$ phases are assumed to be the same (because these data could not obtained) the ratio of $\left(\lambda^{2} V\right)_{\gamma-\beta}$ and $\left(\lambda^{2} V\right)_{\gamma \cdot \beta}$ is 2.22 from equation (1), where the values of the parameters involved are given later in this discussion section. This ratio shows good agreement with the experimental ratio of 2.33 from Table 8 .

Table 8 The $\lambda^{2} \mathrm{~V}$ values in this study compared to other systems [16]

\begin{tabular}{|c||c|c|c|c|c|c|}
\hline System & $\gamma^{\prime}+\beta(2 \mathrm{P}-\mathrm{L})$ & $\gamma+\beta(3 \mathrm{P}-\mathrm{L})$ & $\mathrm{Fe}-\mathrm{C}$ & $\mathrm{Fe}-\mathrm{Fe}{ }_{3} \mathrm{C}$ & $\mathrm{Al}-\mathrm{A} l_{2} \mathrm{Cu}$ & $\mathrm{Sn}-\mathrm{Pb}$ \\
\hline$\lambda^{2} \mathrm{~V}\left(\mu \mathrm{m}^{3}-\mathrm{S}\right)$ & 3264 & 1398 & 460 & 410 & 103 & 35 \\
\hline
\end{tabular}

It is intersting to find that the spacings of the equilibrium $\gamma^{\prime}+\beta$ eutectic and the $\gamma+\beta$ meta-stable eutectic, are unusually large compared with conventional eutectic spacings. The values of $\lambda^{2} \mathrm{~V}$ in $\gamma^{\prime}+\beta$ and $\gamma+\beta$ eutectics are 10-100 times higher than in the Fe-C and $\mathrm{Sn}-\mathrm{Pb}$ 


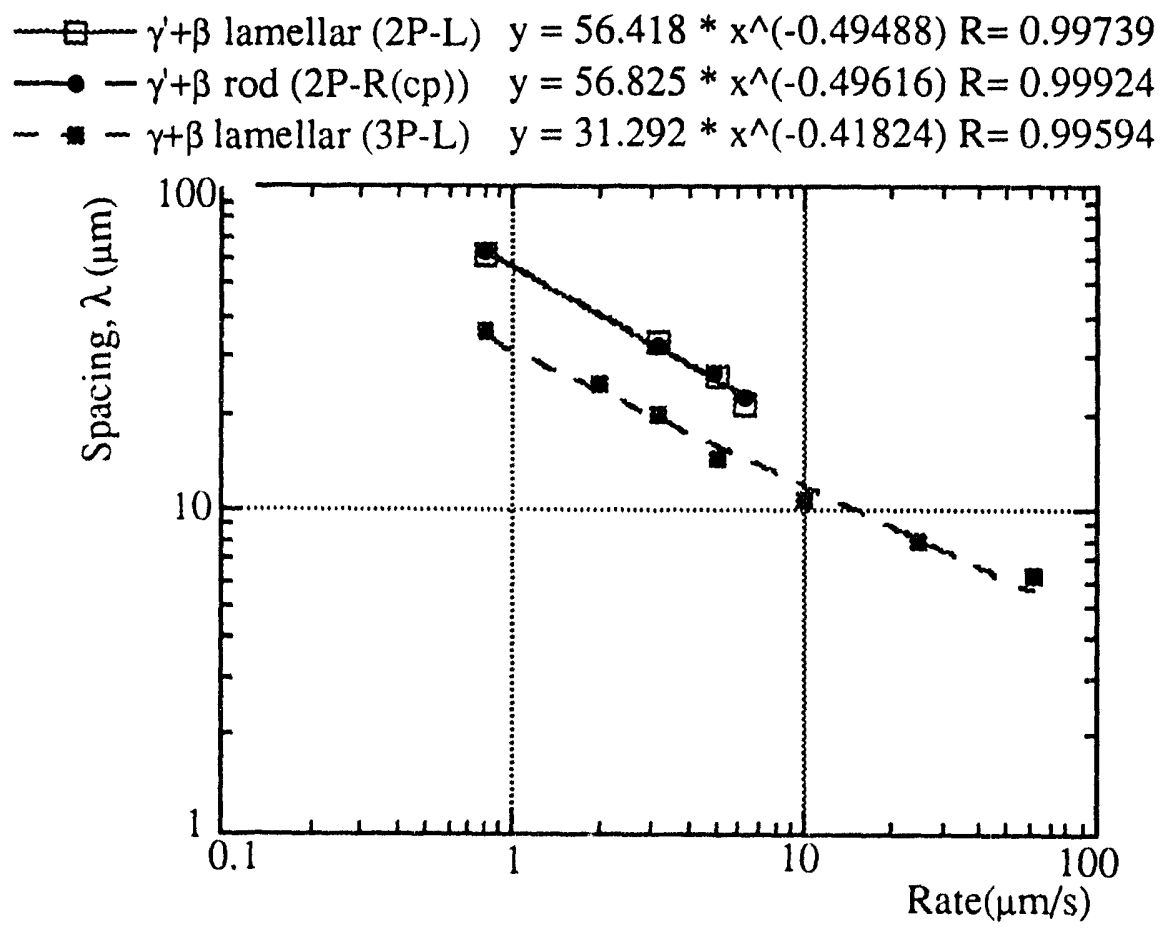

Figure 22. The relation between the eutectic spacing and rate in the equilibrium $\gamma^{\prime}+\beta$ eutectic and the metastable $\gamma+\beta$ eutectic 
systems in Table 8. The mutual liquid diffusion coefficient, $D_{1}$, is suspected to be high because the eutectic transformation is mainly controlled by diffusion. The $D_{1}$ value could be deduced in this experiment by the critical supercooling criterion for the off-eutectic planar interface[15],

$$
\mathrm{D}=\mathrm{m}_{\beta} \mathrm{V}_{\mathrm{cr}}\left(\mathrm{C}_{\mathrm{E}}-\mathrm{C}_{\mathrm{s}}^{*}\right) / \mathrm{G}
$$

where $m_{\beta}$ is the slope of the $\beta$ liquidus, $V_{c r}$ is the critical velocity, $C_{E}$ is the eutectic composition, $\mathrm{C}_{\mathrm{S}}{ }^{*}$ is the solid composition at the solid-liquid interface, and $\mathrm{G}$ is the temperature gradient in the liquid. The $\gamma^{\prime}+\beta$ off-eutectic planar interface changed to the dendritic interface at growth rates between $6.3 \mu \mathrm{m} / \mathrm{s}$ and $10 \mu \mathrm{m} / \mathrm{s}$ in the Al 25 alloy and between $0.8 \mu \mathrm{m} / \mathrm{s}$ and 3.2 $\mu \mathrm{m} / \mathrm{s}$ in the Al 26 alloy in Table 1. The Cs* at $6.3 \mu \mathrm{m} / \mathrm{s}$ of Al 25 alloy was 25 at.\% Al and that at $0.8 \mu \mathrm{m} / \mathrm{s}$ of Al 26 alloy was 26.8 at.\% Al in Table 5. The Cs* at $10 \mu \mathrm{m} / \mathrm{s}$ of Al 25 alloy and $3.2 \mu \mathrm{m} / \mathrm{s}$ of Al 26 alloy were assumed as 25 and 26 at.\% Al. The temperature gradient in the liquid was $2.5^{\circ} \mathrm{C} / \mathrm{mm}$ in these rate ranges $(0.8-10 \mu \mathrm{m} / \mathrm{s})$ as shown in the Appendix. The diffusion coefficients were obtained from equation (2) and these are compared with literature data in Table 9. There is reasonable agreement and the $D$ values are on the order of $10^{-4}$

Table 9. Estimated diffusion coefficient in liquid by the critical supercooling criterion for $\gamma^{\prime}+\beta$ eutectic and comparison with the other data in solidification of nickel-base superalloys

\begin{tabular}{|l|l|l|l|}
\hline Alloy & $V_{c}(\mu \mathrm{m} / \mathrm{s})$ & $D\left(\mathrm{~cm}^{2} / \mathrm{s}\right)$ & Reference \\
\hline \hline Al 25 & $6.3-10$ & $1.4 \times 10^{-4}-2.2 \times 10^{-4}$ & This study \\
\hline Al 26 & $0.8-3.2$ & $9.8 \times 10^{-5}-2.5 \times 10^{-4}$ & This study \\
\hline MAR-M246 & $<1.67$ & $8.3 \times 10^{-5}$ & {$[14]$} \\
\hline MAR-M200 & 1 & $1.4 \times 10^{-4}$ & {$[15]$} \\
\hline
\end{tabular}

$\mathrm{cm}^{2} / \mathrm{s}$. This value is around 10 times higher than usual liquid diffusion coefficients $\left(1-5 \times 10^{-5}\right.$ $\mathrm{cm}^{2} / \mathrm{s}$ ). This high diffusion coefficient in the liquid produces the large spacings of the eutectic structures in this system. Between the $\gamma^{\prime}+\beta$ and $\gamma+\beta$ eutectics, the $\lambda^{2} V$ value of $\gamma+\beta$ eutectic is 
about 2 times smaller than that of the $\gamma^{\prime}+\beta$ eutectic. When the $C_{0}$ in the equation(1) is considered the larger value of $C_{0}$ in the $\gamma+\beta$ eutectic may be the main reason of the smaller value of $\lambda^{2} V$ in the $\gamma+\beta$ eutectic. 


\section{DISCUSSION}

\section{Metastable $\gamma+\beta$ Eutectic}

The experimental results found evidence that the 3P-L eutectic is the metastable $\gamma+\beta$ eutectic initially and the $\gamma^{\prime}$ forms between these two phases at the solid-liquid interface during the quenching procedure. This $\gamma^{\prime}$ layer grows between the $\gamma$ and $\beta$ phases and finally changes to the $\gamma^{\prime}$ single phase $3.5 \mathrm{~mm}$ below the solid-liquid interface in Exp.\#131(Al 24.5(II)-0.8 $\mu \mathrm{m} / \mathrm{s}-\mathrm{Ga}-\mathrm{In})$, as shown in Figure 23.

Suppose that the eutectic at the growth front in the 3P-L structure actually consisted of only $\gamma+\beta$ plates and the $\gamma^{\prime}$ plates seen at room temperature have formed by layer growth on cooling down to room temperature. In this case, the $\gamma$ growth into the $\gamma$ and $\beta$ phases in the $\gamma+\beta$ eutectic could be calculated by a diffusion model. From the solution of Wagner[19], the $\gamma$ formation between the $\beta$ and $\gamma$ phases was obtained, see the schematic diagram is shown in Figure 24,

$$
\begin{aligned}
& \mathrm{Z}_{\beta}=2 \mathrm{a} \sqrt{D_{Y} t} \quad \text { (3) and } \frac{C_{3}-C_{2}}{C_{4}-C_{3}}=\sqrt{\pi} a e^{a^{2}}(\operatorname{erf} a+\operatorname{erf} b) \\
& \mathrm{Z}_{\gamma}=2 b \sqrt{D_{\gamma} t} \quad \text { (5) and } \frac{C_{3}-C_{2}}{C_{2}-C_{1}}=\sqrt{\pi} b e^{b^{2}}(\operatorname{erf} a+\operatorname{erf} b)
\end{aligned}
$$

where $a$ and $b$ are constant. The total thickness of $\gamma^{\prime}$ between the $\gamma$ and $\beta$ phases could be expressed as

$$
\mathrm{Z}_{\mathrm{tot}}=\mathrm{Z}_{\beta}+\mathrm{Z}_{\gamma}
$$

The experiment at $0.8 \mu \mathrm{m} / \mathrm{s}$ with the fast quench of Ga-In(Exp.\#131) was analyzed with this diffusion model. The $\gamma$ phase disappeared $2 \mathrm{~mm}$ below the solid-liquid interface as shown in Figure 23(a). Figures 23(b,c, and d) shows the transverse sections from $0.5 \mathrm{~m}$ a to $3.2 \mathrm{~mm}$ belo" the solid-liquid interface where the $\gamma$ grows between the $\gamma$ and $\beta$ phases. All the eutectic phases $(\gamma$ and $\beta$ ) transformed to $\gamma$ 'single phase $3.5 \mathrm{~mm}$ below the solid-liquid interface. The diffusion coefficient, D, in $\gamma^{\prime}$ has been determined as $4 \times 10^{8} \times \exp (-32200 / \mathrm{T})$ 120). The $D$ at the liquid interface $\left(138()^{\circ} \mathrm{C}\right)$ is $1.387 \mu \mathrm{m}^{2} / \mathrm{s}$ and it falls to $1.262 \mu \mathrm{m}^{2} / \mathrm{s} 2 \mathrm{~mm}$ 
a)

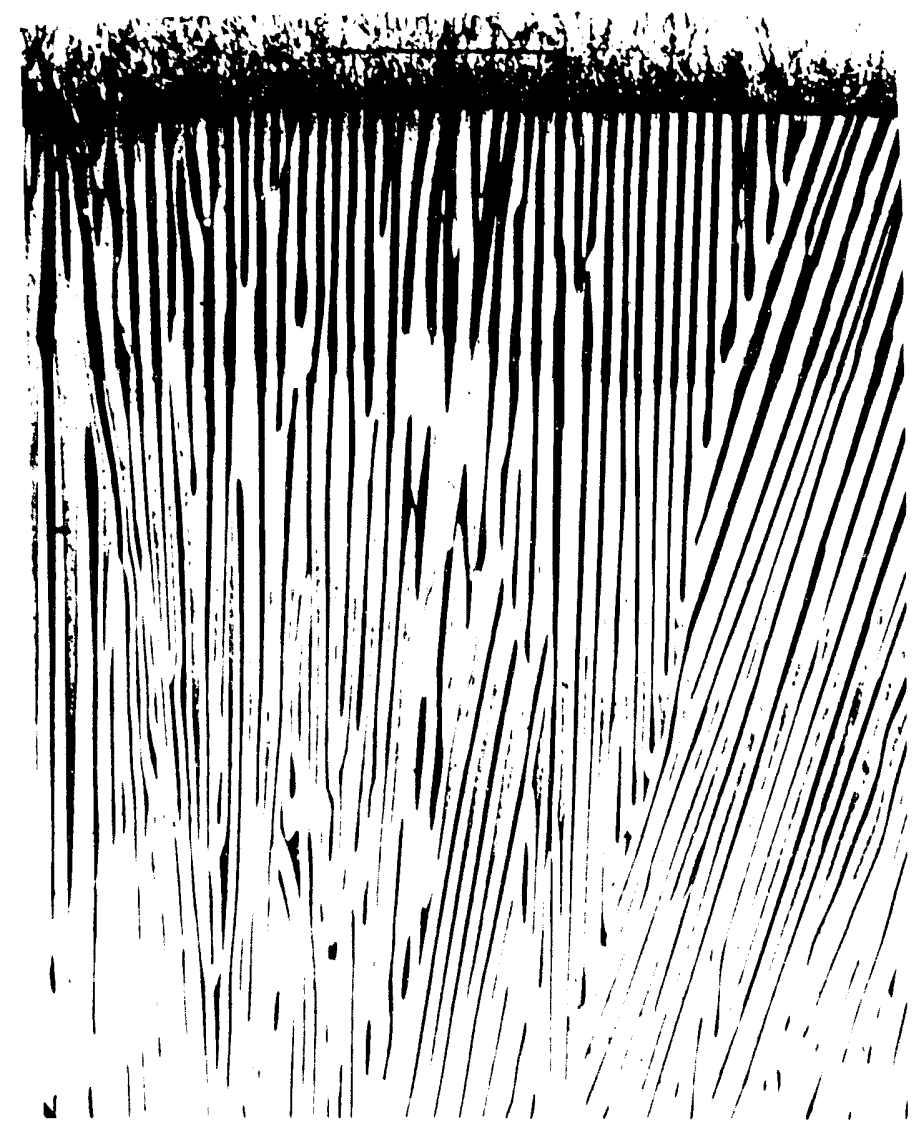

Figure 23. The solid state transformation in the $\gamma+\beta$ eutectic Exp.\#131(Al 24.5-0.8 $\mu \mathrm{m} / \mathrm{s}, \mathrm{Ga}-$ In quench) The eutectic phases change to the $\gamma^{\prime}$ single phase according to the distance from the solid-liquid interface. a) Longitudinal view, $50 \mathrm{x}$ 


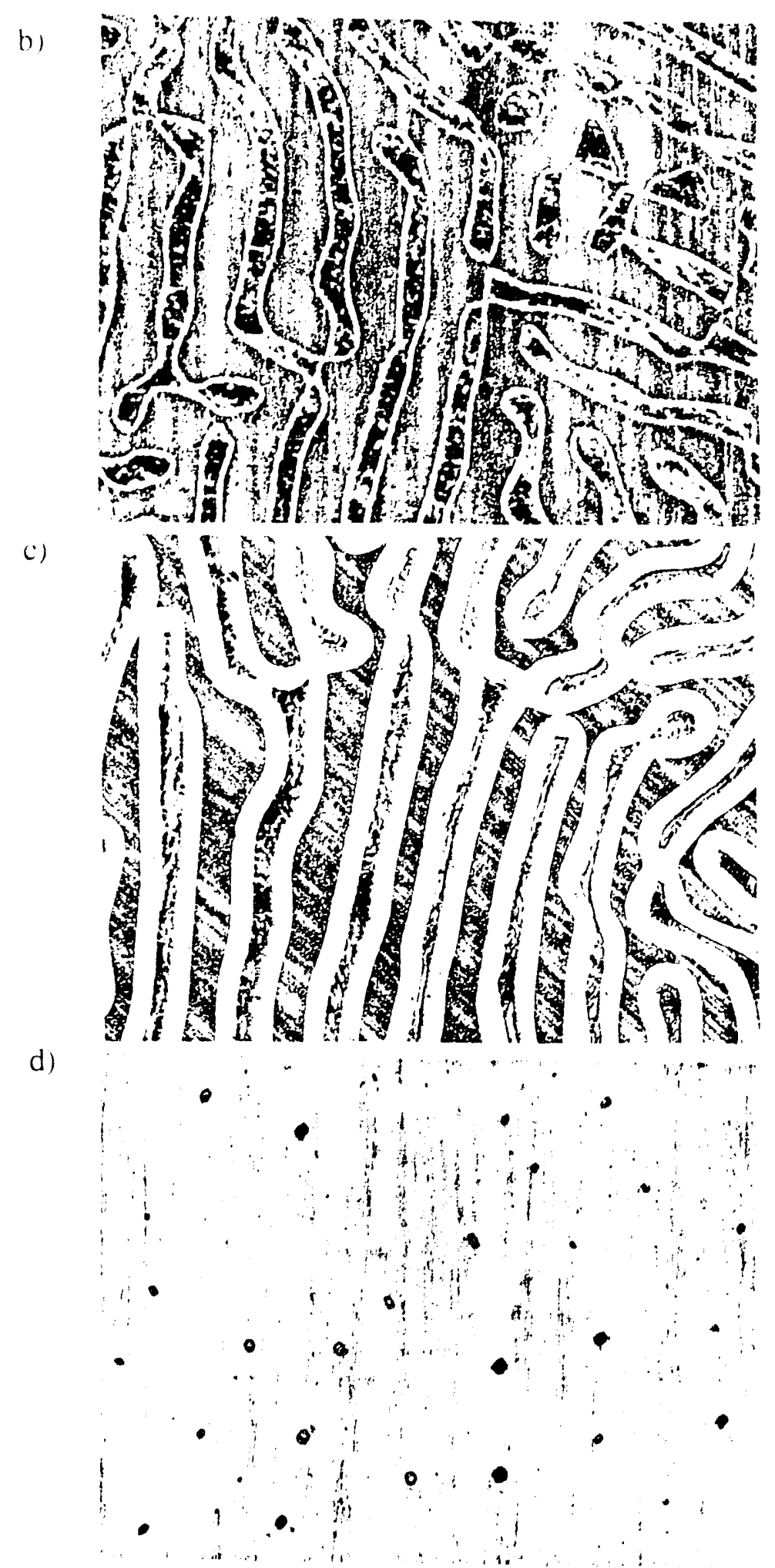

Figure 23. (continued) and b), c), and d) transverse views $(0.5 \mathrm{~mm}, 1 \mathrm{~mm}$, and $3.2 \mathrm{~mm}$ below the solid-liquid interface, $100 \mathrm{x}$ 
a)

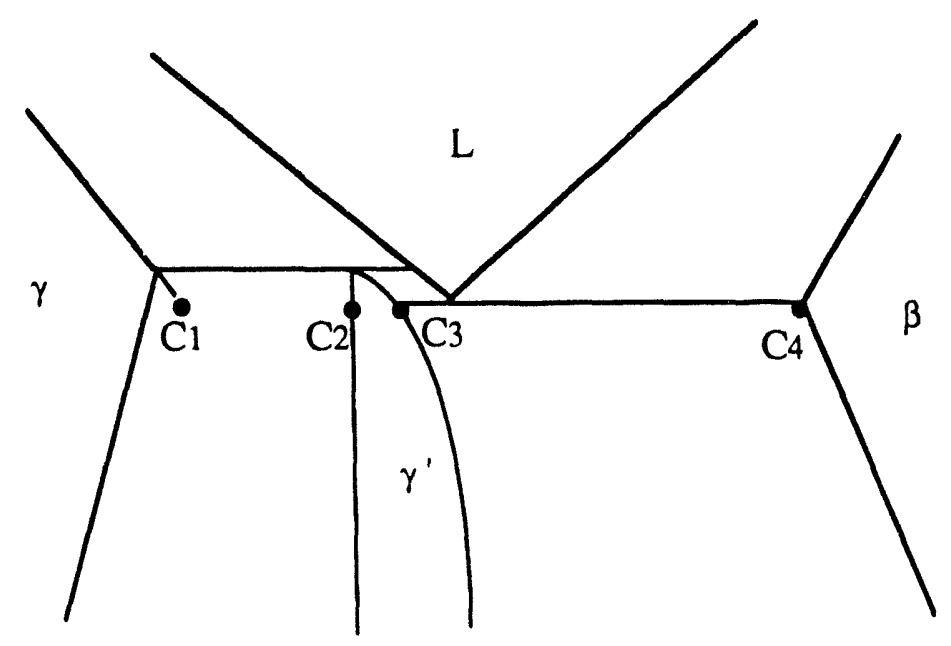

b)

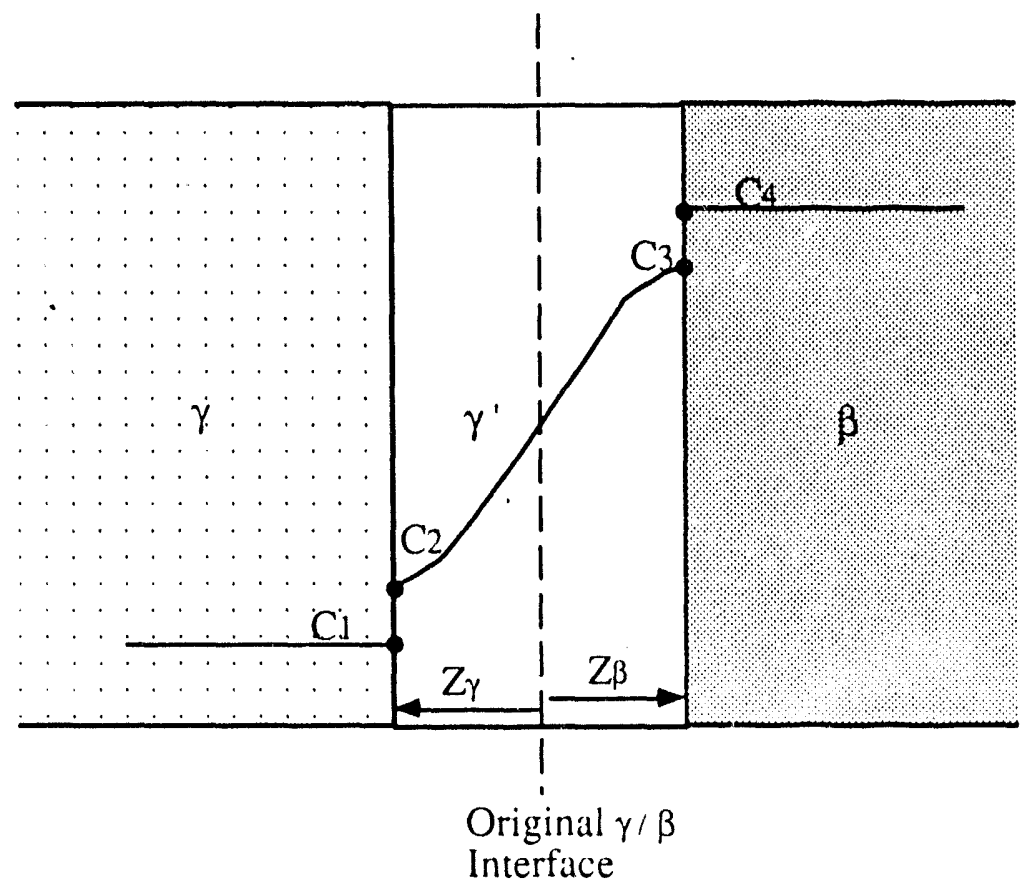

Figure 24. Diffusion in three phase system from Wagner's solution. a) The boundary compositions in the schematic phase diagram and b) concentration distribution at each phase 
below the interface $\left(1372^{\circ} \mathrm{C}\right)$, where the solid temperature gradient was taken to be $4.2^{\circ} \mathrm{C} / \mathrm{mm}$ using the data in the Appendix. Hence D was assumed constant as $1.3 \mu \mathrm{m}^{2} / \mathrm{s}$ in the following calculations.

A problem was found in applying this diffusion model. The $Z_{\beta}$ and $Z_{\gamma}$ depend on the width of $\gamma^{\prime}$ phase field $\left(\Delta C=\right.$ the composition range of $\left(C_{3}-C_{2}\right)$ in the phase diagram. However, this $\Delta C$ is not well established, while the $C_{1}$ and $C_{4}$ are well established, see Figure 9. Different $\Delta C^{\prime}$ s were applied to Equations (4) and (6) from 0.3 to 0.1 at $1380^{\circ} \mathrm{C}$ and the constants a and $\mathrm{b}$ were obtained on each $\Delta \mathrm{C}$ as shown in Table 10. The $\mathrm{Z}_{\text {tot }}$ was calculated

Table 10. The calculated values of constants, $a$ and $b$, from Equations (4) and (6) for several expected phase diagrams

\begin{tabular}{|c|c|c|c|c|c|c|c|c|}
\hline Temp. $\left({ }^{\circ} \mathrm{C}\right)$ & Diagram \# & $\mathrm{C}_{1}$ & $\mathrm{C}_{2}$ & $\mathrm{C}_{3}$ & $\mathrm{C}_{4}$ & $\Delta \mathrm{C}_{(2-3)}$ & $\mathrm{a}$ & $\mathrm{b}$ \\
\hline \hline \multirow{3}{*}{1380} & 1 & 21.6 & 23.3 & 23.4 & 28.2 & 0.1 & 0.052 & 0.146 \\
\cline { 2 - 9 } & 2 & $"$ & 23.2 & 23.4 & $"$ & 0.2 & 0.073 & 0.212 \\
\cline { 2 - 9 } & 3 & $"$ & 23.1 & 23.4 & $"$ & 0.3 & 0.089 & 0.267 \\
\hline \multirow{3}{*}{1300} & 4 & 21.6 & 23.8 & 23.6 & 28.2 & 2.5 & 0.532 & 0.482 \\
\cline { 2 - 9 } & 5 & 19.5 & 23.8 & 26.3 & 31.0 & 2.5 & 0.345 & 0.370 \\
\hline
\end{tabular}

from the solid-liquid interface position to $2 \mathrm{~mm}$ below the solid-liquid interface by Equation (7) and the results are given in Figure 25(a). Another assumption should be involved because the $\Delta \mathrm{C}$ changes according to the temperature. The $\Delta \mathrm{C}$ needs to be constant between the interface temperature $\left(1380^{\circ} \mathrm{C}\right)$ and the position $2 \mathrm{~mm}$ below solid-liquid interface $\left(1372^{\circ} \mathrm{C}\right)$ for application of equations (3-6). The thicknesses of $\gamma^{\prime}$ were measured from the solid-liquid interface to the position $2 \mathrm{~mm}$ below the solid-liquid interface and applied to the estimated thickness of $\gamma^{\prime}$. The diffusion model gives roughly the right magnitudes on the distance of disappearance. However, the thickness of $\gamma^{\prime}$ in the initial $480 \mu \mathrm{m}$ was constant at $2 \mu \mathrm{m}$. After this constant region the thickness of $\gamma^{\prime}$ was increased in a similar manner to the estimated 


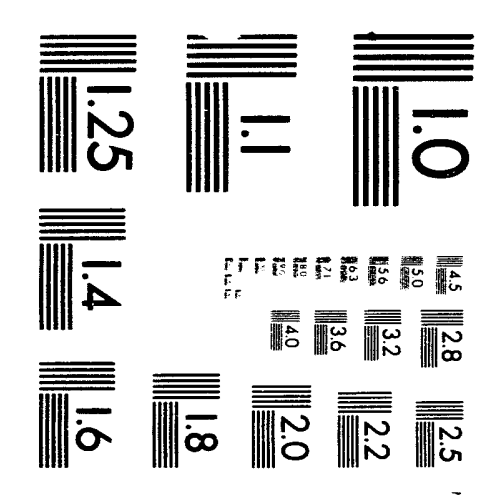



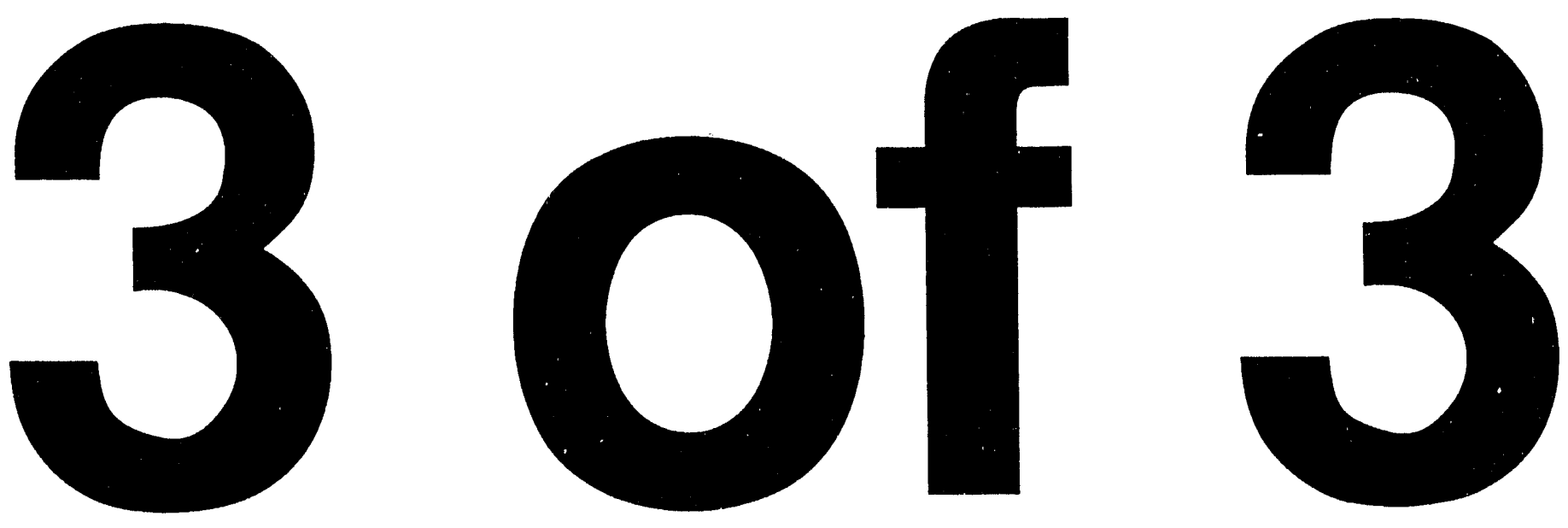
a)

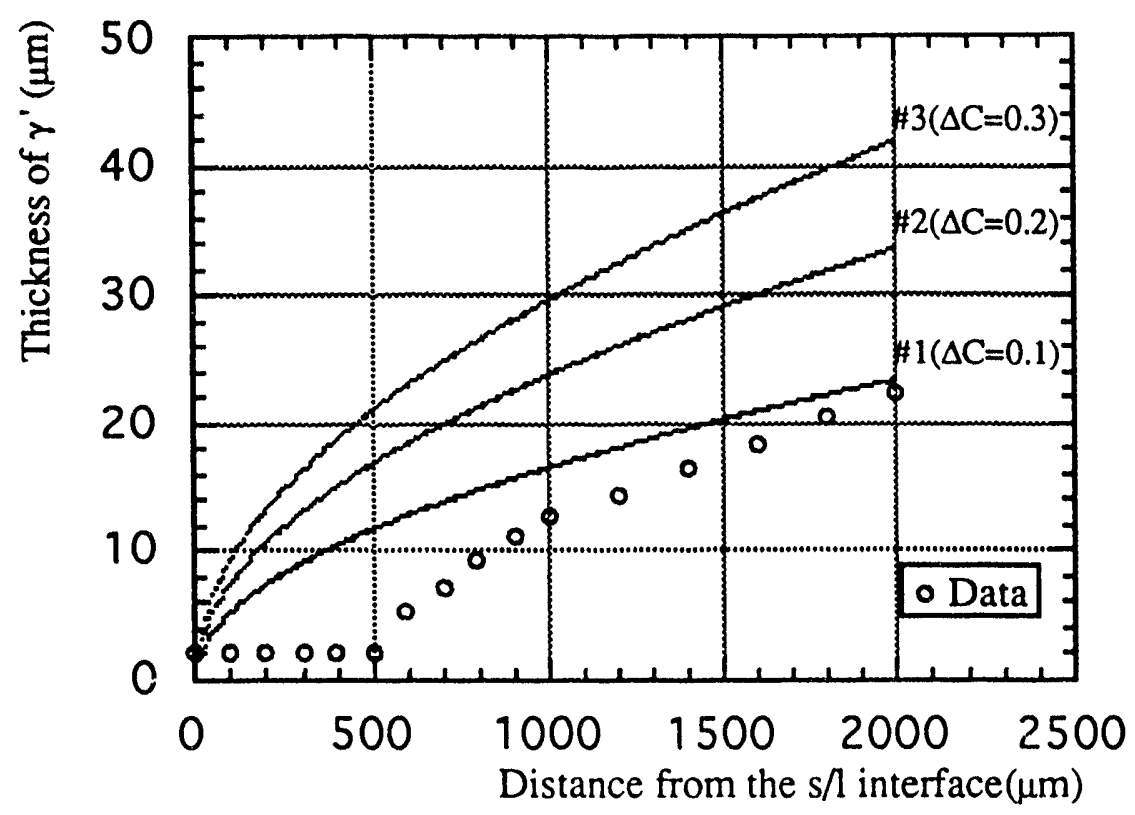

b)

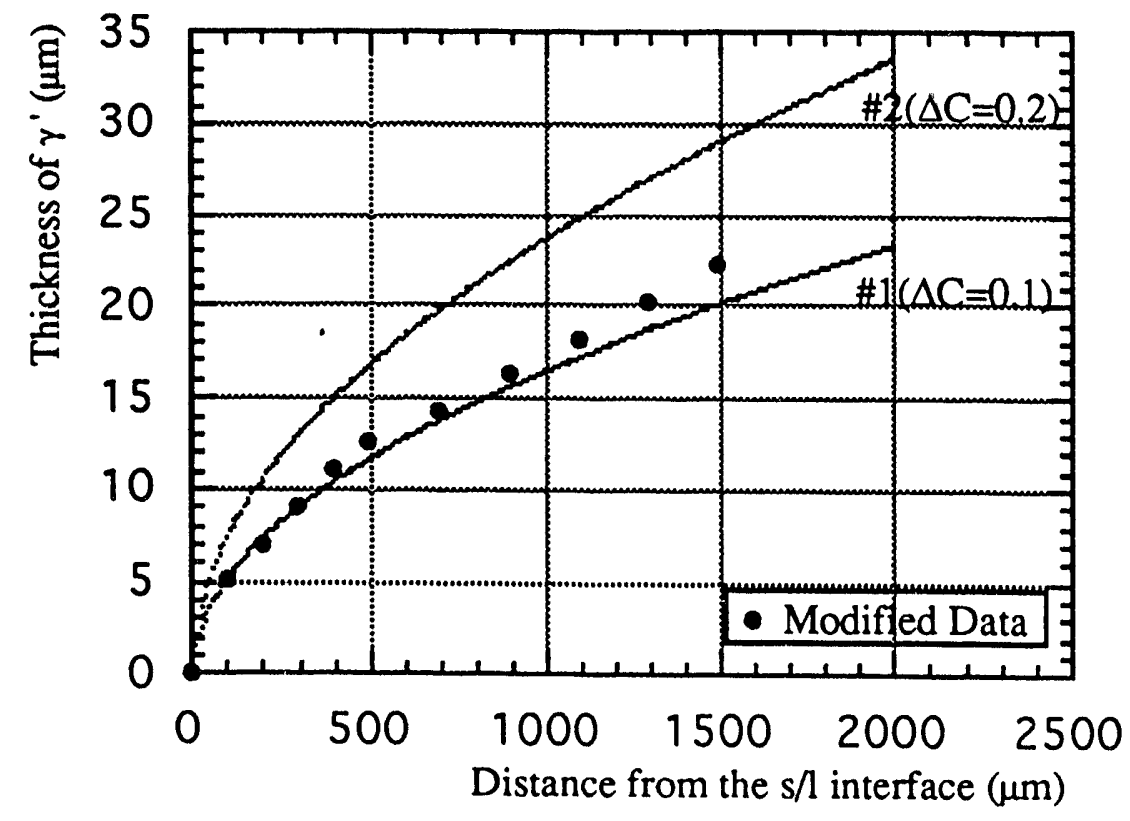

Figure 25. a) The growth of the $\gamma^{\prime}$ phase between the $\gamma$ and $\beta$ phases by the diffusion model and the experimental data. b) The modified data was applied to the diffusion model 
values. It is thought that the $\gamma^{\prime}$ in the constant region might result from the solid state transformation upon quenching. This idea is suggested by Table 6 where the thickness of $\gamma^{\prime}$ is reduced at faster quenching. Also, the fact that even with the Ga-In the $\gamma^{\prime}$ precipitates formed in all the $\beta$ and $\gamma$ phases, indicates that there is a strong possibility that a significant formation of $\gamma^{\prime}$ occurs during the quenching procedure.

An important question arises as to why the $\gamma^{\prime}$ starts to form $480 \mu \mathrm{m}$ below the solidliquid interface if the initial constant $\gamma^{\prime}$ is really formed on the quenching procedure. The diffusion model assumed that the $\gamma^{\prime}$ phase nucleates right at, or just below, the solid-liquid front. If it needs some time to nucleate, the $\gamma^{\prime}$ might start to form $480 \mu \mathrm{m}$ below solid-liquid interface which corresponds to 600 seconds and the $2 \mu \mathrm{m}$ thick $\gamma^{\prime}$ might be nucleated and formed on the quenching. The driving force for formation of $\gamma^{\prime}$ must be high on quenching because it is more easy to form $\gamma^{\prime}$ at lower temperature and the composition range of $\gamma^{\prime}$ is larger at lower temperature. If the average phase transformation temperature on quenching is assumed as about $1300^{\circ} \mathrm{C}$, the $\Delta \mathrm{C}_{(\mathrm{C} 3-\mathrm{C} 2)}$ is 2.5 at\% in the phase diagram of Figure 9 . The following calculation shows that the $\Delta \mathrm{C}$ may cause formation of $\gamma^{\prime}$ in only 1 second. The $\gamma^{\prime}$ layer formation during the quench procedure is estimated in Figure 26 where the constants are calculated at $1300^{\circ} \mathrm{C}$ in Table 10 . The phase diagram \#5 in Table 10 corresponds to the $C_{1}$ and $\mathrm{C}_{4}$ equilibrium compositions at $1300^{\circ} \mathrm{C}$. However, if the compositons of $\gamma$ and $\beta$ phases in the eutectic are maintained upon quenching at their $1380^{\circ} \mathrm{C}$ values, then the phase diagam \#4 is more probable than the phase diagram \#5. In either case the $\gamma^{\prime}$ grows at least $1 \mu \mathrm{m}$ within $1 \mathrm{sec}$, as shown in Figure 26.

After this analysis was completed on exp 131 it was decided that an experiment with a faster quench rate might be able to show the absence of the $\gamma^{\prime}$ phase at the quench interface. Hence, the experiments in the smaller tube diameters, exps 133 and 134 were carried out as has been described above. Figure 18(a) show the $\gamma^{\prime}$ phase beginning to form as a layer between the $\beta$ and $\gamma$ phases at a distance of $250 \mu \mathrm{m}$ below the interface. The higher magnification 


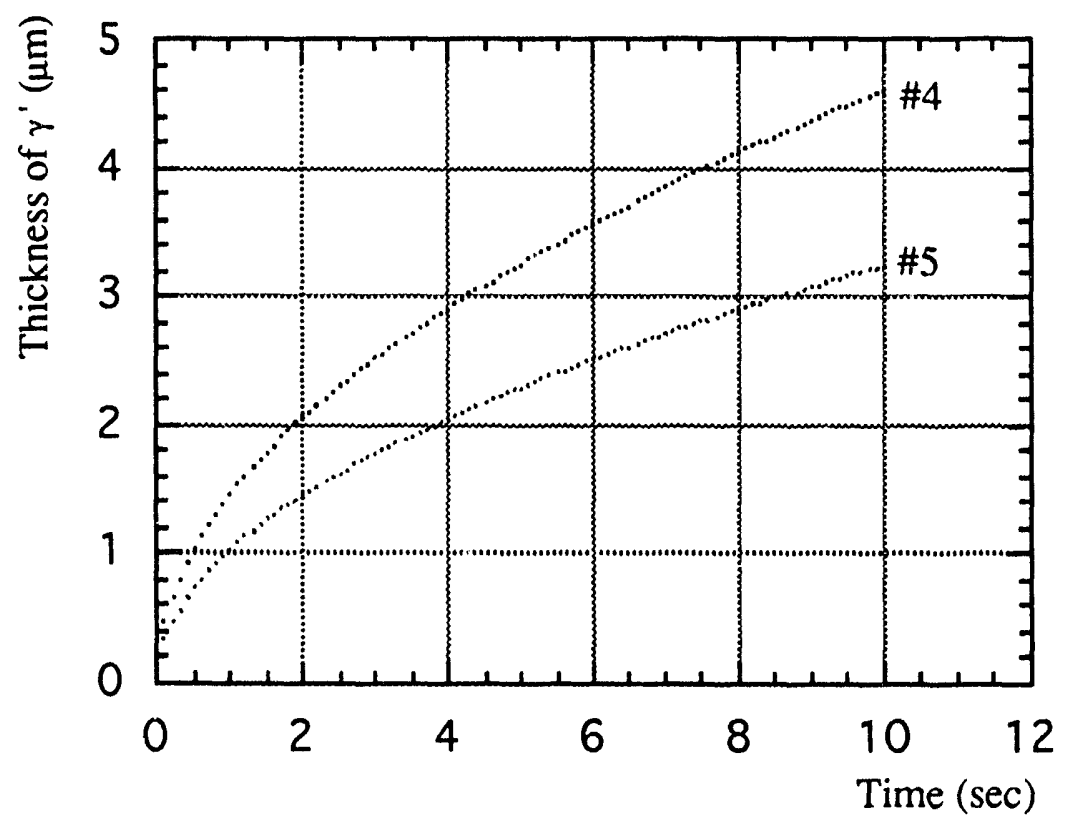

Figure 26. The expected $\gamma^{\prime}$ layer growth between the $\gamma$ and $\beta$ phases during the quench proceauie by the diffusion model. The compositions of phase diagram \# are designated in Table 10 
picture of Figure 18(b) reveals that the $\gamma^{\prime}$ phase is not absent above this point. Although it is only $1 \mu \mathrm{m}$ thick, compared to the $2 \mu \mathrm{m}$ for the slower quench of the $5 \mathrm{~mm}$ I.D.tube experiment of Figure 23, it has not gone to zero, as had been hoped would be the case. If one looks at the quench interface region on the liquid side of the advancing solid/liquid interface in Figure 18(b), one also sees a thin layer of the $\gamma^{\prime}$ phase. This $\gamma^{\prime}$ phase must have formed upon quenching and since it is of roughly the same thickness as that of the $\gamma^{\prime}$ phase in the first 250 $\mu \mathrm{m}$ below the quench interface, it provides fairly conclusive evidence that the $1 \mu \mathrm{m}$ layer of the $\gamma^{\prime}$ phase has formed during the quench procedure.

A question occurs as to why the lengths of the constant $\gamma^{\prime}$ layers, $\delta_{\text {const } \gamma}$, are different in Exp.\#131 $(480 \mu \mathrm{m})$ and Exp.\#134 $(250 \mu \mathrm{m})$ at the same rate of $0.8 \mu \mathrm{m} / \mathrm{s}$. It was found that the solidification rate was not controlled precisely in the most recent experiment of the smaller diameter tube( $3 \mathrm{~mm}$ I.D.), Exps.\#134. The run distance was $10 \mathrm{~mm}$ but the actual directional solidification distance was $6.5 \mathrm{~mm}$. The solidification rates were usually well controlled in normal solidification procedure in the $5 \mathrm{~mm}$ I.D. tube but the difference between the run distance and solidification distance become significant at high solidification fractions. For this solidification system it is thought that the solidification rate is not controlled precisely in the initial and final transition solidification fractions. The difference between the run and solidification distances is expected to be due to the initial transition in Exp.\#134. This effect might be a reason for the different $\delta_{\text {const } y}$ lengths. The $\delta_{\text {const } \gamma}$ lengths were measured at actual average solidification rates in Al 24.5(II) alloy and the results are shown in Table 11 and Figure 27 where the $\delta_{\text {const }}$ is proportional to the actual soldification rate. This result provides additional evidence that the constant $\gamma^{\prime}$ layer at the solid-liquid interface is formed on quenching and the $\gamma^{\prime}$ layer starts to grow after the $\delta_{\text {const }} \gamma^{\prime}$ which corresponds to about $500-600$ sec. In order to nucleate the $\gamma^{\prime}$ phase between the $\beta$ and $\gamma$ phases, nucleation time (about 500$600 \mathrm{sec}$ ) is thought to be required and then the diffusion model could be applied. 
Table 11. Relation between the solidification rate, $V$, and the length of the constant $\gamma^{\prime}$

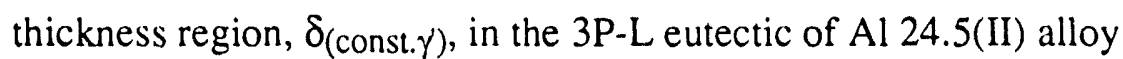

\begin{tabular}{|c|c|c|c|c|c|c|c|}
\hline Exp.\# & $\begin{array}{c}\text { Rate } \\
(\mu \mathrm{m} / \mathrm{s})\end{array}$ & $\begin{array}{l}\text { Actual Avg } \\
\text { Rate }(\mu \mathrm{m} / \mathrm{s})\end{array}$ & fs & $\begin{array}{c}\text { Sample dia. } \\
(\mathrm{mm})\end{array}$ & $\begin{array}{l}\text { Quench } \\
\text { Media }\end{array}$ & $\begin{array}{c}\delta_{\left(\text {const } \gamma^{\prime}\right)} \\
(\mu \mathrm{m})\end{array}$ & $\begin{array}{c}\text { Time for } \delta \\
(\mathrm{sec})\end{array}$ \\
\hline $117(115)$ & 0.8 & 0.8 & 0.40 & 5 & Water & 500 & 625 \\
\hline 131 & 0.8 & 0.8 & 0.30 & 5 & Ga-In & 480 & 600 \\
\hline 134 & 0.8 & 0.5 & 0.22 & 3 & Ga-In & 250 & 500 \\
\hline 118 & 3.2 & 3.2 & 0.47 & 5 & Water & 1900 & 594 \\
\hline
\end{tabular}

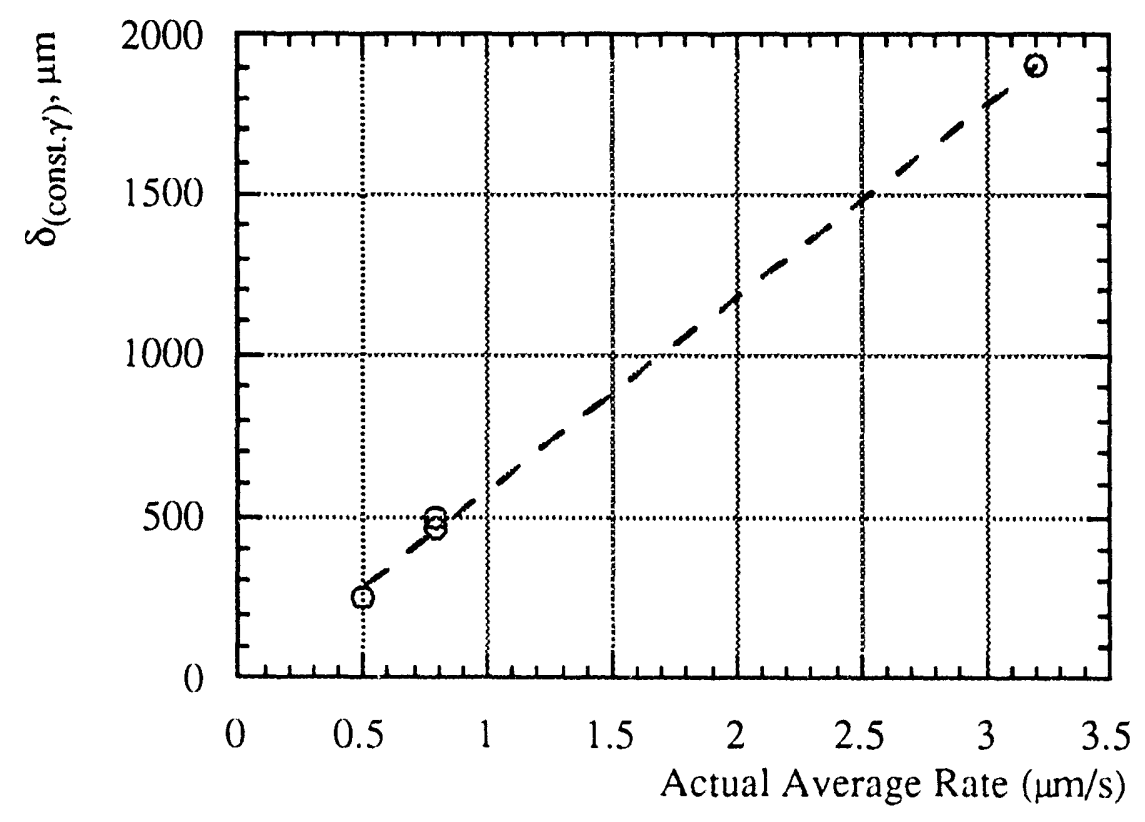

Figure 27. Relation between the actual solidification rate and the length of the constant $\gamma^{\prime}$ layer ir Al 24.5(II) alloy 
If the $y^{\prime}$ forms from the position $480 \mu \mathrm{m}$ below the solid-liquid interface in Exp.\#131, the diffusion model may be applied from that position. The thickness data were modified by removing the $480 \mu \mathrm{m}$ of $2 \mu \mathrm{m}$ thick $\gamma^{\prime}$, which is suspected to be formed on the quenching procedure, and the results are shown in Fig 25(b). This modified data fit well to the phase diagram \# 1 which correspond to $\Delta \mathrm{C}=0.1$. This result indicates that the composition range of $\gamma^{\prime}$ in the phase diagram is about 0.1 at.\% at the eutectic temperature.

\section{Condition for Metastable Eutectics}

\section{Metastable $\gamma+\beta$ eutectic}

The metastable $\gamma+\beta$ eutectic, which was thought to be the 3P-L eutectic initially, was formed in the impurity added Al 23.5(I) alloy and at high soiidification rates of 25 and 61 $\mu \mathrm{m} / \mathrm{s}$. The impurities, $\mathrm{Fe}$ and $\mathrm{Cr}$, may suppress the $\gamma^{\prime}$ liquidus line such that the metastable $\gamma$ liquidus line extrapolates to a eutectic point so close to the $\gamma^{\prime}+\beta$ eutectic point that the metastable $\gamma+\beta$ eutectic forms. The results of chemical analyses at the 3P-L eutectic(now $\gamma+\beta$ eutectic) support this idea because the eutectic compositions are lower in the $\gamma+\beta$ eutectic. The compositions of the meta-siable $\gamma+\beta$ eutectic were 24.4 at.\% Al $1 \mathrm{~mm}$ below solid-liquid interface and 24.3 at. \% $\mathrm{Al}$ at just after the $\gamma$ planar, as shown in Table 5. These compositions are less than the usual $\gamma^{\prime}+\beta$ eutectic compositions.

At high solidification rates of 25 and $61 \mu \mathrm{m} / \mathrm{s}$ the eutectic structure was always the metastable $\gamma+\beta$ eutectic. The eutectic interface temperature $\left(T_{i}(E)\right)$ is given as [21],

$$
T_{i}(E)=T_{E}-K_{E} V^{1 / 2}
$$

where $T_{E}$ is the eutectic temperature, $K_{E}$ is constant, and $V$ is solidification rate. This equation shows that the undercooling of eutectic at $61 \mu \mathrm{m} / \mathrm{s}$ is higher than that of low rates. In fast growth rate which induces more undercooling in the eutectic, the metastable eutectic might be possible because of the closeness of the eutectic and peritectic temperatures in this system. 
It was found that the metastable $\gamma+\beta$ eutectic was formed by impurity effect to suppress the equilibrium liquidus of $\gamma^{\prime}$ in contaminated alloy of $\mathrm{Al}$ 23.5(I) and the velocity effect to stabilize the metastable eutectic phases. Also, the $\gamma+\beta$ eutectic was found at low rates in the eutectic composition alloys of $\mathrm{Al} 24.5$ (I) and (II). In Al 24.5(II) alloy all $\gamma+\beta$ metastable eutectic was formed at $0.8 \mu \mathrm{m} / \mathrm{s}$ and this eutectic changes to $3 \mathrm{P}-\mathrm{R}(\mathrm{c})$ and $2 \mathrm{P}-\mathrm{R}(\mathrm{c})$ at 3,5 , and $10 \mu \mathrm{m} / \mathrm{s}$ (see Figure 11). These results indicates that the composition variations from center to edge of the rod cause the eutectic morphology changes. Figure 11(b) shows the structure changes by formation of $\gamma^{\prime}$ from the $\gamma+\beta$ eutectic at the center region to the $2 \mathrm{P}-\mathrm{R}(\mathrm{c})$ to the $3 \mathrm{P}-$ $\mathrm{R}(\mathrm{c})$ at the edge region. It was proved that the composition of center region was higher than that of edge region in Table 5. The cellular type eutectics(2P-R(c) and 3P-R(c)) were formed at compositions slightly lower then the eutectic composition in Figure 15. The metastable $\gamma+\beta$ eutectic is thought to be also formed at low rate $(0.8 \mu \mathrm{m} / \mathrm{s})$ at the composition of 24.7 at $\% \mathrm{Al}$, which is slightly hyper-eutectic for the phase diagram of Figure 9.

\section{Three phase cellular type(3P-R(c)) eutectic}

The 3P-L eutectic was proved to really be the $\gamma+\beta$ metastable eutectic. However, three phases, $\gamma+\gamma^{\prime}+\beta$, were formed at the solid-liquid interface in the 3P-R(c) eutectic. This structure appears to be a mixed structure of the $\gamma+\gamma^{\prime}$ coupled peritectic and the $\gamma^{\prime}+\beta$ rod eutectic. Experimental results showed that the 3P-R(c) eutectic formed at compositions slightly lower than the eutectic composition. However, it was difficult to determine more complete conditions for the 3P-R(c) eutectic without further study on conditions of formation of the coupled peritectic. Important questions should be solved, such as the mechanism for formation of the coupled peritectic, which will help model the conditions required for the coupled peritectic growth with the eutectic at the same growth front. The dependence upon the $\mathrm{G} / \mathrm{V}$ value is suspected as well as the dependence of small composition variations for the conditions for the 3P-R(c) eutectic. Further experiments are required to characterize the 3P-R(c) eutectic 
and the coupled peritectic. These experiments will involve controlling the temperature gradient and precise composition analysis.

\section{Convection Effect at the Eutectic Growth Front}

Figure 11 and 12 showed that the center and edge eutectic structures were different. Also, the $\mathrm{Al}$ compositions at the edge were 0.1-0.2 at.\% lower than those at the center in all three analyzed samples( Exps.\# 61, 118, and 110) in Table 5, where the eutectic structures were the 3P-L $(\gamma+\beta$ eutectic $)$ and $3 \mathrm{P}-\mathrm{R}(\mathrm{c})$ eutectics. When the expected natural convection pattern due to thermal gradients is considered, as shown in Figure 28, a little Ni-rich solute should be rejected from the eutectic growth front to give lower $\mathrm{Al}$ content in the edge side. Solute convection is expected to be small due to the small composition differences between solid and liquid in eutectic growth. Additional evidence was found that the Ni-rich solute was rejected from the $\gamma+\beta$ eutectic(3P-L) growth front. Figure 13 shows that the structure of the beginning of eutectic formation, where $f s=0.47$, is the meta-stable $\gamma+\beta$ eutectic $(3 \mathrm{P}-\mathrm{L})$ and it changes to the $3 P-R(c)$ eutectic at $f s=0.6$. Figure 15 showed that the meta-stable $\gamma+\beta$ eutectic(3P-L) formed at higher $\mathrm{Al}$ concentrations $(24.7$ at $\% \mathrm{Al})$ than the $3 \mathrm{P}-\mathrm{R}(\mathrm{c})$ or $2 \mathrm{P}-\mathrm{R}(\mathrm{c})$ eutectics $(24.5-24.6$ at $\% \mathrm{Al})$.

The existance of a diffusion boundary layer at the eutectic solid-liquid interface during the directional solidification of the eutectics was predicted by the diffusion equation [22]. This boundary layer might result in the radial composition changes. The Jackson-Hunt model [22] was modified and well expressed by Magnin and Trivedi [16]. The general solution of the solute field in front of the planar eutectic solid-liquid interface moving at a constant velocity is expressed as

$$
\mathrm{C}=\mathrm{C}_{\mathfrak{c}}+\Delta \mathrm{C}_{\infty}+\mathrm{B}_{0} \exp \left(-\frac{V}{D} \mathrm{z}\right)+\sum_{n=1}^{\infty} B_{n} \exp \left(-n \frac{2 \pi}{\lambda}\right) \cos \left(n \frac{2 \pi}{\lambda} x\right)
$$

where $B_{0}$ and $B_{n}$ are Fourier coefficients, $\lambda$ is the lamellar spacing, $C_{c}$ is the eutectic 


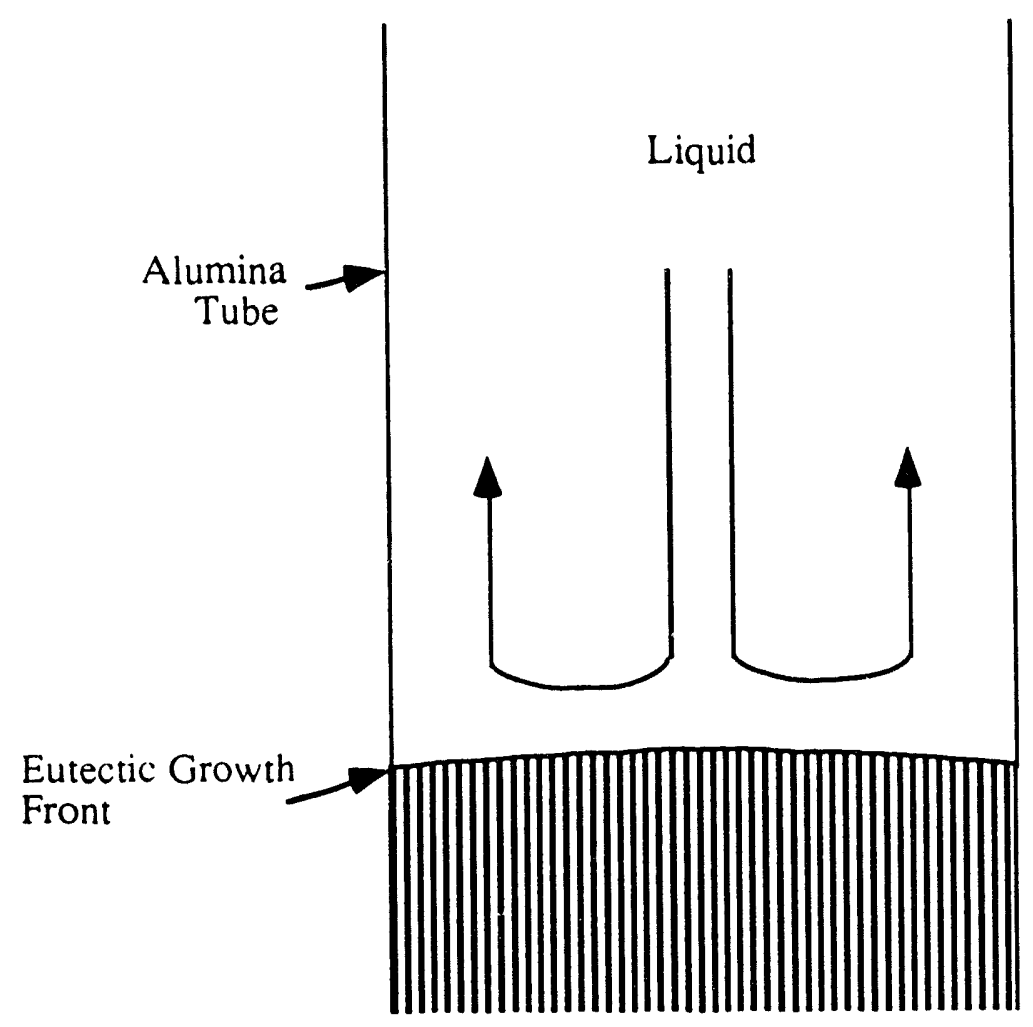

Figure 28. Expected natural convection in front of the eutectic growth front due to thermal gradients 
composition, and $\Delta \mathrm{C}_{\infty}$ is the difference between the initial composition of the liquid and $\mathrm{C}_{\mathrm{e}}$ (see Figure 29). The solute balance at the solid-liquid interface leads to

$$
\begin{array}{rlrl}
-\left.\mathrm{D} \frac{\partial C}{\partial z}\right|_{z=0} & =V\left(C_{e}+\delta C\right)\left(1-k_{\alpha}\right) & & \text { for } \alpha \text { phase } \\
& =-V\left(1-C_{e}-\delta C\right)\left(1-k_{\beta}\right) & \text { for } \beta \text { phase }
\end{array}
$$

where the $k_{\alpha}$ and $k_{\beta}$ are the solute distribution coefficients of $\alpha$ and $\beta$ phases. By applying the boundary condition of equation (10) to the general solution of equation (9), $B_{0}$ and $B_{n}$ are obtained as

$$
\begin{aligned}
& \mathrm{B}_{0}=-\Delta \mathrm{C}_{\infty}+\delta \mathrm{C} \\
& \mathrm{B}_{\mathrm{n}}=\frac{\lambda}{(n \pi)^{2}} \frac{V}{D} C_{0} \sin \left(n \pi f_{\alpha}\right)
\end{aligned}
$$

where the the density difference between two phases is neglected. As shown in Figure 29 the $\mathrm{B}_{0}$ term represents the average composition of a boundary layer in front of the solid-liquid interface which is different than the average composition in the liquid far from the interface. Therefore, the value of $\mathrm{B}_{0}$ is an important factor to determine whether $\mathrm{Al}$-rich or Ni-rich solute is rejected from the eutectic solid-liquid interface in the $\mathrm{Ni}$-Al system. The composition difference between the average liquid composition at the eutectic solid-liquid interface and the eutectic composition, $\delta \mathrm{C}$, could be obtained by the relation between the growth undercooling and the interface thermodynamic equilibrium[16].

$$
\begin{gathered}
\delta C=\frac{\Delta T}{\bar{m}}\left\{\frac{f_{\alpha} m_{\beta}-f_{\beta}\left|m_{\alpha}\right|}{\left|m_{\alpha}\right|+m_{\beta}}+\frac{\left(\Gamma_{\beta} \sin \theta_{\beta} / m_{\beta}-\Gamma_{\alpha} \sin \theta_{\alpha} /\left|m_{\alpha}\right|\right) /\left(\phi^{2}+1\right)}{\Gamma_{\alpha} \sin \theta_{\alpha} / f_{\alpha}\left|m_{\alpha}\right|+\Gamma_{\beta} \sin \theta_{\beta} / f_{\beta} m_{\beta}}\right\} \\
\Delta \mathrm{T}=\left(\phi+\frac{1}{\phi}\right) \sqrt{K_{1} K_{2} V}
\end{gathered}
$$

Theoretical $\delta C$ values could be determined by the equation (12) but it is difficult to find data for the $\Gamma$ and $\theta$ terms. These two terms are related to the surface entergy and entropy which are not known. For the Gibbs-Thomson coefficient, $\Gamma$, the following empirical relationship is 


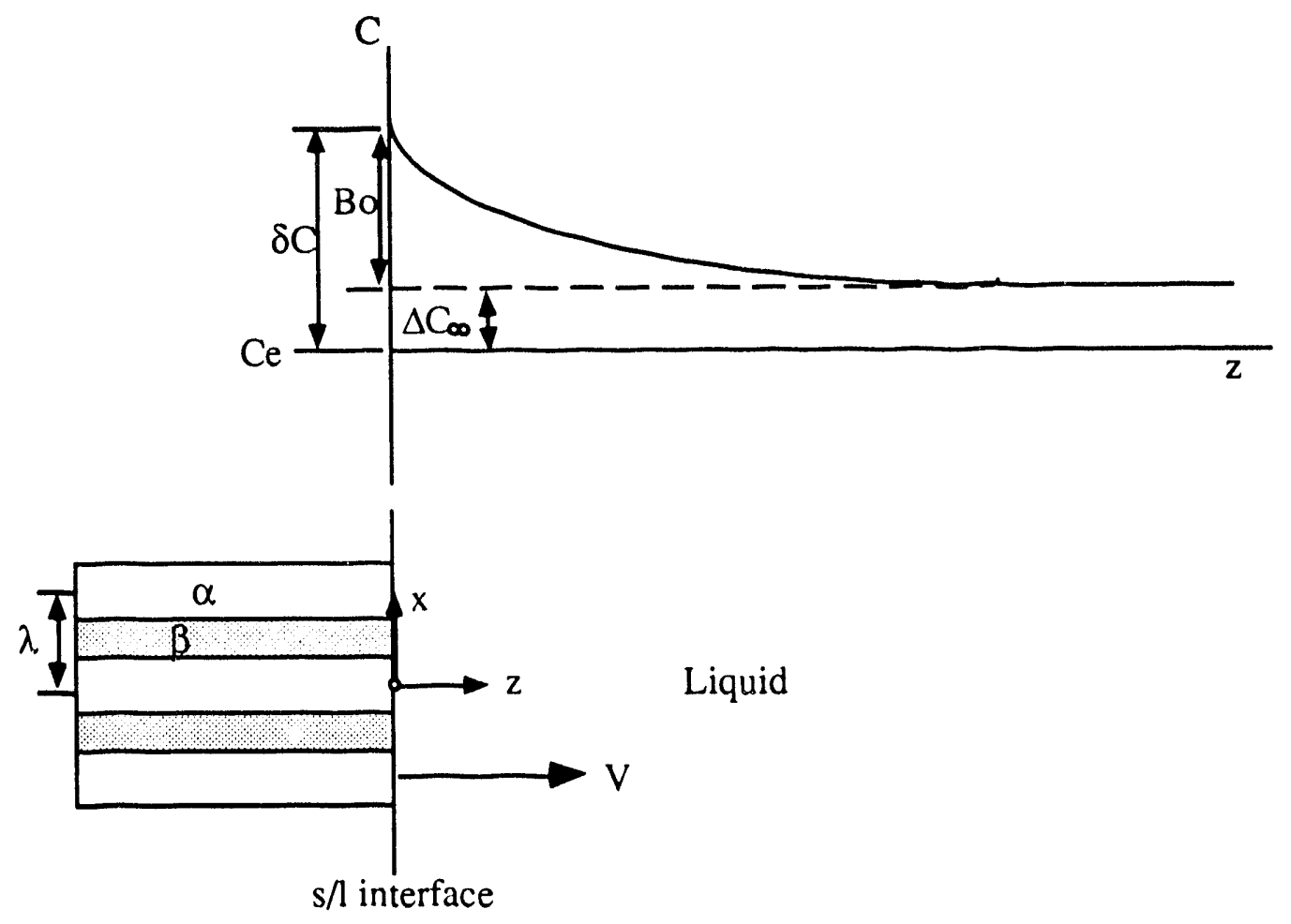

Figure 29. Schematic diagrams of the average composition profile at the eutectic solid-liquid interface and the geometry of the lamellar eutectic microstructure 
used[16],

$$
\Gamma=5.45 \times 10^{-9} \mathrm{~T}_{\mathrm{f}} \mathrm{Vm}^{1 / 3}
$$

where $T_{f}$ is the melting temperature of the phase and $V_{m}$ is the molecular volume of the phase. Although this relationship is not very accurate, the values obtained are reasonable approximations. In order to estimate the contact angles at the three phase junction, the surface energies at the solid-liquid interface are required. The entropy can be estimated by the following equation[23].

$$
\Delta \mathrm{S}_{\mathrm{f}}=\frac{R T_{E}\left(C_{S}-C_{L}\right)}{m V_{s}\left(1-C_{L}\right) C_{L}}
$$

where $R$ is a gas constant, $V_{S}$ is $g$ molecular volume, $C_{L}$ and $C_{S}$ are the compostions of solid and liquid at the solid-liquid interface, and $m$ is the liquidus slope. Because $\Gamma=\gamma_{\mathrm{s}^{\prime}} / \Delta \mathrm{S}_{\mathrm{f}}$, the surface energy, $\gamma_{\mathrm{SL}}$, could be obtained by equations (13) and (14). From the force balance of the surface energies of the eutectic phases, $\gamma_{\alpha \mathrm{LL}} \cos \theta_{\alpha}=\gamma_{\beta \mathrm{L}} \cos \theta_{\beta}$ (neglected a torque term), and the experimantal values of $\lambda^{2} \mathrm{~V}$ with the equation (1), $\left(\lambda^{2} \mathrm{~V}\right)_{\mathrm{E}: \mathrm{P}}=\mathrm{K}_{2} / \mathrm{K}_{1}$, the contact angles may be obtained. Using the involved parameters, the $\delta C$ 's in the $\gamma+\beta$ and $\gamma^{\prime}+\beta$ eutectics could be obtained by equation (9) as shown in Table 12 , where $m_{\gamma}$ and $m_{\gamma}$ are $6.5^{\circ} \mathrm{K} / a t \%$ and $m_{\beta}$ is 14 ${ }^{\circ} \mathrm{K} / \mathrm{at} \%$ from Table 6 in Paper $\mathrm{I}$, and $\mathrm{D}$ is $1.5 \times 10^{-8} \mathrm{~m}^{2} / \mathrm{s}$ from Table 9.

Table 12. The calculation of $\delta C$ and involved parameters in the $\gamma+\beta$ and $\gamma^{\prime}+\beta$ eutectics at 1 $\mu \mathrm{m} / \mathrm{s}$.

\begin{tabular}{|c|c|c|c|c|c|c|c|c|c|c|}
\hline Eutectic & $\mathfrak{f}_{\alpha}$ & $\mathrm{f}_{\beta}$ & $\mathrm{C}_{0}$ & $\mathrm{P}$ & $\Gamma_{\alpha}\left(10^{7} \mathrm{mk}\right)$ & $\Gamma_{\beta}\left(10^{7} \mathrm{mk}\right)$ & $\theta_{\alpha}{ }^{\circ}$ & $\theta_{\beta^{\circ}}$ & $\mathrm{K}_{2} / \mathrm{K}_{1}\left(\mathrm{~m}^{3} / \mathrm{s}\right)$ & $\delta C(\mathrm{a} \%)$ \\
\hline \hline$\gamma+\beta$ & 0.55 & 0.45 & 6.7 & 0.03332 & 1.8 & 2.0 & 54.7 & 1.7 & $1.39 \times 10^{-15}$ & -0.00013 \\
\hline$\gamma^{\prime}+\beta$ & 0.79 & 0.21 & 4.7 & 0.01760 & 1.7 & 2.0 & 55.2 & 23.4 & $3.28 \times 10^{-15}$ & +0.00122 \\
\hline
\end{tabular}

The $\delta C$ shows a negative value in the $\gamma+\beta$ eutectic and a positive value in the $\gamma^{\prime}+\beta$ eutectic. The amount of solute at the solid-liquid interface is determined by $B_{0}$ as shown in Figure 29. The equation (11) shows that the sign of $B_{0}$ is determed by not only $\delta C$ but also $\Delta C_{\infty}$. The 
$\gamma+\beta$ eutectic is expected to be formed in the slightly hyper-eutectic compositions as shown in Figure 15 and TaL'e 5. This result means that $\Delta \mathrm{C}_{\infty}$ is positive in the $\gamma+\beta$ eutectic(3P-L). Because $\Delta \mathrm{C}_{\infty}$ is expicted to be positive and $\delta \mathrm{C}$ is negative, it is seen from equation (11) that $\mathrm{B}_{0}$ must be negative, which would result in the rejection of $\mathrm{Ni}$-rich solute from the eutectic solid-liquid interface. The experimental results, that the Ni-rich solute is rejected from the growth front of the 3P-L( $\gamma+\beta)$ and 3P-R(c) eutectics, agree with this thoretical expectation. However, the thoretical value of $\delta \mathrm{C}$ is not well established because the enthalpies, surface energies, and contact angles have not been estimated. Because the $\Gamma$ and $\theta$ terms are very important to decide the value of $\delta \mathrm{C}$ in the equation (9), experimental measurements of these terms are required. Nevertheless, the boundary layer in the eutectic growth front provides a reasonable explanation for the radial composition variations in the $3 \mathrm{P}-\mathrm{L}(\gamma+\beta$ eutectic) eutectic and the eutectic structure changes from the initial $\gamma+\beta$ eutectic to the $3 P-R(c)$. 


\section{CONCLUSIONS}

1. The $\gamma^{\prime}+\beta$ eutectic was expected to form in $\mathrm{Ni}-\mathrm{Al}$ alloys based on the phase diagram in Part I, but several unusual eutectic structures were found near the eutectic compositions, as well as a meta-stable eutectic. In hyper eutectic alloys the equilibrium $\gamma^{\prime}+\beta$ eutectics of lamellar and rod morphologies, coded as $2 \mathrm{P}-\mathrm{L}$ and $2 \mathrm{P}-\mathrm{R}(\mathrm{cp})$, were found at low solidification rates. In the eutectic composition and hypo-eutectic composition alloys, three phase $(\gamma+\beta+\gamma)$ eutectics were also found as the lamellar and rod types at low rates, where the rod type eutectics appeared as a cellular morphology with the eutectic formed around the $\gamma^{\prime}$ cells, coded as $3 \mathrm{P}-\mathrm{L}$ and $3 P-R(c)$. Two phase $\left(\gamma^{\prime}+\beta\right)$ eutectic was also found in hypo-eutectic alloys but it was a cellular type of rod eutectic, coded as $2 \mathrm{P}-\mathrm{R}(\mathrm{c})$. At relative fast rate of $61 \mu \mathrm{m} / \mathrm{s}$ all the eutectic was the $3 \mathrm{P}-\mathrm{L}$ eutectic.

2. The classical eutectic morphologies of the $2 \mathrm{P}-\mathrm{L}$ and $2 \mathrm{P}-\mathrm{R}(\mathrm{cp})$ eutectics were found as the planar off-eutectic morphologies at low rates in hyper-eutectic alloys, but the longitudial morphology appeared to be the classical cellular shape and the spacings were unusually large for a eutectic structure. The study of the mystery interface \#I in the 3P-R(c) in Paper III proved that the original solid-liquid interface was planar rather than cellular. The diffusion coefficient in the liquid, $D_{l}$, was estimated by the critical supercooling criterion of the offeutectic planar interface and it was about 10 times larger than the usual $D_{1}$ values of $10^{-5} \mathrm{~cm}^{2} / \mathrm{s}$. The large value of $D_{1}$ in this system is thought to produce the large spacing of the eutectic in this system.

3. The 3P-L eutectic structure was shown to be the metastable $\gamma+\beta$ eutectic by the quench rate control experiments. The quench rates were increased by using Ga-In as a quench media and by using a smaller diameter container tube. A $\gamma^{\prime}$ layer was formed between the $\gamma$ 
and $\beta$ phases in the $\gamma+\beta$ eutectic during the quench procedure, which resulted in the presence of three phases $\left(\gamma+\gamma^{\prime}+\beta\right)$ at the growth front in the samples examined after quenching. A diffusion model of this layer growth gave reasonablely good agreement with the kinetics of the layer formation below the advancing solid/liquid interface. The model supports a composition difference between the $\mathrm{C}_{2}-\mathrm{C}_{3}$ compositions, at the eutectic temperature shown in Figure 24(a), of about 0.1 at $\%$.

4. The meta-stable $\gamma+\beta$ eutectic was induced by the presence of impurities, ie. Fe and $\mathrm{Cr}$, and by high solidification rates. Also, this metastable eutectic was found at the low solidification rate of $0.8 \mu \mathrm{m} / \mathrm{s}$ at only one composition, 24.7 at\% $\mathrm{Al}$, which is slightly hypereutectic for the phase diagram of Figure 9.

5. The cellular types of eutectics $(2 \mathrm{P}-\mathrm{R}(\mathrm{c})$ and $3 \mathrm{P}-\mathrm{R}(\mathrm{c}))$ were found at the compositions slightly lower than the eutectic composition. In this celluar type it is thought that the 2 or 3 phases of the eutectics grow cooperatively with the $\gamma^{\prime}$ cells as a cylindrical plane around these cells. However, it was strange to find three phases at the growth front in the 3P-R(c) eutectic. The 3P-R(c) structue appears to be a mixed structure of the $\gamma+\gamma^{\prime}$ coupled peritectic and the $\gamma+\beta$ rod eutectic. In order to understand this $3 P-R(c)$ eutectic, the study of the coupled peritectic should be continued. 


\section{REFERENCES}

1. Lemkey, F.D.; Hertzberg, R.W; Ford, J.A. Met. Trans., 1965, 233,334.

2. Ashbrook, R.L.; Wallace, J.F. Met. Trans., 1966,236,670

3. Lemkey, F.D.; Thomson, E.R. Met.Trans., 1971, 2, 1537

4. Crossman, F.W.; Yue, A.S. Met. Trans., 1971, 2, 1545

5. David,I.G.; Double, D.D. in "Solidification and Casting of Metals," The Metal Society, London, 1979, 94.

6. Elmalallawy, N.A.; Farag, M.M. in "Solidification and Casting of Metals," The Metal Society, London, 1979, 106.

7. McLean, M. in "Solidification and Casting of Metals," The Metal Society, London, 1979, 65.

8. Livinston, I.D., et. al. Acta Metall. 970, 18, 399.

9. Lee, J.H.; MS Thesis, ISU, Ames, IA, 1989.

10. Bottinger, W.J. Met. Trans., 1974, 5, 2023.

11. Ostrowski, A. ; Langer, E. in "Solidification and casting of Metals," The Metal Society, London, 1979, 65.

12. Hillert, M. in "Solidification and casting of Metals," The Metal Society, London, 1979, 81.

13. Bradley, A.J. J. Iron Steel Inst, 1949, 19.

14. Taylor, A.; Floyd, R.W. J. Inst. Met., 1952, 81, 451.

15. Verhoeven J. D. "Fundamentals of Physical metallurgy," John wiley \& Sons, New York, NY, 1975, 277

16. Magnin P.; Trivedi, R. K. Acta Metall., 1991, 39(4), 453.

17. Quested, P.N.; McLean, M. Mat. Sci. Eng., 1984, 65, 171.

18. Tien, J. K.; Gamble, R.P. Mat. Sci. Eng. , 1971,8, 152.

19. Jost, W. "Diffusion in Solids, Liquids, Gases," Academic Press, New York, NY, 1953, 74.

20. Janssen, M.M.P. Met. Trans., 1973, 4, 1623.

21. Trivedi, R.; Kurz, W. in "Solidification Processing of Eutectic Alloys," The 
Metallurgyical Society, 1988, 3.

22. Jackson, K.A.; Hunt, J.D. Trans. Metall. AIME, 1966, 236, 1129.

23. Gunduz, M.; Hunt, J.D. Acta Metall., 1985, 33(9), 1651. 


\section{GENERAL SUMMARY}

A directional solidification study was carried out to distinguish two contradictory phase diagrams near $\mathrm{Ni}_{3} \mathrm{Al}$ compositions, a eutectic reaction between the $\beta$ and $\gamma^{\prime}$ phases and between the $\gamma$ and $\gamma^{\prime}$ phases. Directional solidification studies, that preserve the microstructures formed at the solidification front, have presented conclusive evidence that the eutectic reaction occurs between the $\beta$ and $\gamma^{\prime}$ phase and a peritectic composition lying at lower Al concentrations than the eutectic composition. The solid state phase boundaries between the $\beta, \gamma^{\prime}$, and $\gamma$ phases at temperatures just below the freezing range was determined with diffusion couple experiments and these experiments are consistent with the result of the directional solidification experiments.

In the course of the study on the directional solidification of $\mathrm{Ni}-\mathrm{Al}$ alloys having compositions near $\mathrm{Ni}_{3} \mathrm{Al}$, some unusual etching characteristics which made phase identification difficult were found. A significant variation in gray level is observed in single-phase $\gamma^{\prime}$ grains as well as the $\gamma$ grains which include $\gamma$ ' precipitates. It has been shown with aid of the SACP technique in an SEM that a strong correlation exists with gray level and crystallographic orientation. The $<111>$ orientations produce dark gray levels, $<001>$ orientations produce light gray levels and a gradual transition in gray levels occurs over intermediate orientations across the entire standard triangle of orientations from $<001>$ to $<111>$. It was demonstrated that the cause of the gray level variation was the formation of $<001>$ microfacets in the $\gamma^{\prime}$ phase produced on etching, which in turn generate micro-surface morphologies that maximize light scattering for $<111>$ orientations. These results are important for characterizing the relative surface energies of etched $\mathrm{Ni}_{3} \mathrm{Al}$ surfaces and they have also been very helpful in the interpretation of $\mathrm{Ni}_{3} \mathrm{Al}$ samples which undergo recrystallization during the cool-down phase of directional solidification. 
The usual quenched solid-liquid interfaces showed two $\gamma^{\prime} / \gamma^{\prime}$ interfaces that lie parallel to the solid-liquid growth front of $\gamma^{\prime}$ or $\gamma+\gamma^{\prime}$. The origin of the interfaces was not well understood and these have been termed mystery interfaces. Experiments were carried out to investigate the role of quench rate on the formation of mystery interfaces by utilizing TEM and SEM analyses to identify the nature of the interfaces. The experiments which varied the quench rate have shown that upon quenching the mystery interfaces are induced by formation of the $\gamma^{\prime}$ bands. TEM and SEM analyses have proved that there are no crystallographic orientations and structure changes across these boundaries. Careful EDS experiments using low voltage excitation to minimize beam spreading in an SEM as well as thin foil TEM experiments have shown that the mystery interfaces result from a small composition variations at the interfaces. The occurrence of a mystery interface at the $\gamma+\gamma^{\prime}$ coupled growth front is useful because it outlines the position of the $\gamma^{\prime}$ growth front at the instant of the quench.

The eutectic solidification study confirms that the equilibrium eutectic is $\gamma^{\prime}+\beta$. However, several unusual eutectic structures as well as a meta-stable eutectic have been found near the eutectic composition. In hyper-eutectic alloys the equilibrium $\gamma^{\prime}+\beta$ eutectics of lamellar and rod morphologies, coded as 2P-L and 2P-R(cp), were found at low solidification rates. At the eutectic composition and hyper-eutectic composition alloys, three phase $\left(\gamma^{\prime}+\gamma+\beta\right)$ eutectics were also found as the lamellar and rod types at low rates, where the rod type eutectics appeared as a cellular morphology with the eutectic formed around the $\gamma^{\prime}$ cells, coded as $3 \mathrm{P}-\mathrm{L}$ and $3 \mathrm{P}-\mathrm{R}(\mathrm{c})$. Two phase $\left(\gamma^{\prime}+\beta\right)$ eutectic was also found in hypo-eutectic alloys but it was cellular type rod eutectic, coded as $2 \mathrm{P}-\mathrm{R}(\mathrm{c})$. At relatively fast rate of $61 \mu \mathrm{m} / \mathrm{s}$ all the eutectic was the 3P-L eutectic. The 2P-L and 2P-R(cp) eutectics were classical eutectic morphologies but those spacings were unusually large for a eutectic structure. The large spacing of the eutectic was shown to be due to the large value of diffusion coefficient in the liquid, $D_{l}$, which was measured by the critical supercooling criterion of the off-eutectic planar interface. The 3P-L eutectic structure was shown to be the meta-stable $\gamma+\beta$ eutectic with 
quench rate control experiments. The meta-stable $\gamma+\beta$ eutectic was induced (1) by the presence of impurities, (2) by high solidification rates (above $25 \mu \mathrm{m} / \mathrm{s}$ ), and (3) at low solidification $\operatorname{rate}(0.8 \mu \mathrm{m} / \mathrm{s})$ by controlling compositions to lie near the eutectic composition. The cellular types of eutectics (2P-R(c) and 3P-R(c)) were found at the compositions slightly lower than the eutectic composition. In this cellular type it is thought that 2 or 3 phases of the eutectics grow with the $\gamma^{\prime}$ cells as a cylindrical plane around these cells. However, it was strange to find three phases at the growth front in the 3P-R(c) eutectic. The 3P-R(c) structure appears to be a mixed structure of the $\gamma+\gamma^{\prime}$ coupled peritectic and the $\gamma^{\prime}+\beta$ rod eutectic. The $\gamma+\gamma^{\prime}$ coupled growth was found at low rates in hypo-eutectic alloys, but these experiments are not complete and were not presented here. The possibility of the coupled peritectic has been of special interest in the peritectic system.. In order to understand this 3P-R(c) eutectic, the study of the coupled peritectic should be continued. 


\section{REFERENCES}

1. Hansen, M.; Anderko, K. "Constitution of Binary Alloys," McGraw Hill, New York, N.Y., 1958, 118.

2. Metals Handbook, Vol 8, 8th Edition, ASM International, Metals Park, OH, 1973, 262.

3. Singleton, M. F.; Murray, J. L. ; Nash, P. in "Binary Alloy Phase Diagrams," ASM International, Metals Park, OH, 1986, 140.

4. Schramm, J. Z. Metallk. 1941, 33, 347.

5. Hilpert, K.; Kobertz, D.; Venugopal, V.; Miller, M.; Gerads, H.; Bremer, F. J.; Nickel, H. Z. Naturforsch.,1987, 42a, 1327.

6. Bremer, F. J.; Beyss, M.; Karthaus, E.; Hellwig, A.; Schober,T.; Welter, J. M.; Wenzl, H. J. Crys. Growth, 1988, 87, 185.

7. Lee, J. M.S.Dessertation, Iowa State Univ., Ames, Iowa, 1989. 


\section{ACKNOWLEDGEMENTS}

First of all, I would like to express my sincere appreciation to my major professor Dr. John D. Verhoeven. Dr. Verhoeven gave me interest in the field of metallurgy by his inspiring lectures and allowed me to complete this thesis by his circumspect guidance which I deeply appreciate.

I would like to thank Dr. R.K. Trivedi for invaluable discussion throughout this work and extend my gratitude to Dr. K.R. Hebert, Dr. F.X. Kayser, Dr. S. Bahadur, and Dr. L.S. Chumbley who served and supported as my committee members. Special thanks are extended to Mr. F.A. Schmidt for funding and kind concern, and I appreciate Dr. T. Lograsso for useful advice and suggestions. I am also indebt to Mr. Ed Gibson for technical assistance, Mr. F. Labbs for teaching EDS, Mr. L. Lincoln for preparing alloy samples, Mr. H. Sailsbury for metallography, and MPC Analytical group for chemical analysis. I can not also forget Ms. P. Boone's kind. I wish to express thanks to Ken, Greg, Paul, Park, Kim, Mary, Han, Jeff, and Hwang for their friendship.

Finally, I wish to express my heartful thanks to my parents, wife, and son.

This work was performed at Ames Laboratory under contract no. W-7405-eng-82 with the U.S. Department of Energy. The United States goverment has assigned the DOE Report number IS-T 1653 to this thesis. 


\section{APPENDIX}

\section{Temperature Gradient Measurment}

Temperature grtadient is one of the most important parameters with the velocity to determine the solid-liquid interface morphologies. Using the temperature gradients in the liquid at $61 \mu \mathrm{m} / \mathrm{s}$ the liquidus lines were determined in Paper I. The temperature gradients in the liquid and solid $\left(G_{l}\right.$ and $\left.G_{s}\right)$ were also measured at different velocities, and at several furnace conditions which were obtained accidentally and on purpose. The gradient data, which were measured at several different conditions, provided important information not only to study the directional solidification but also to deduce the thermal conductivity of $\mathrm{Ni}_{3} \mathrm{Al}$.

The temperature gradients were measured as described in Paper I. At three different velocities, 3.2, 25, and $61 \mathrm{~mm} / \mathrm{s}$, the gradients were measured. Those were also measured ar several different furnace conditions. (1) The position of the cold finge was lowered, as shown in Figure 1, which results in lower temperature gradients. (2) The furnace temperatures were reduced from normal furnace temperature 150()$^{\circ} \mathrm{C}$ to $1467^{\circ} \mathrm{C}$ and $1445^{\circ} \mathrm{C}$. (3) During the experiments the furnace was fixed because of a problem in the electrical connections inside the furnace. After that the gradients were increased significantly. The experimental results are shown in Table 1 where low c.f. means that the cold finger was lowered 1 inch and 1500(old) means the furnace condition at $1500^{\circ} \mathrm{C}$ before fixing.

Table 1. Temperature gradients and velocities at different furnace set temperatures

\begin{tabular}{|c|c|c|c|c|c|c|c|c|c|c|}
\hline Furmace Temp. $\left({ }^{\circ} \mathrm{C}\right)$ & \multicolumn{2}{|c|}{$1500)$} & \multicolumn{2}{|c|}{1467} & \multicolumn{2}{|c|}{1445} & 144 (low c.f.) & \multicolumn{1}{|c|}{1500 (old) } \\
\hline $\begin{array}{c}\text { Gradicnt }\left({ }^{\circ} \mathrm{C} / \mathrm{cm}\right) \\
\text { Rate }(\mu \mathrm{m} / \mathrm{s})\end{array}$ & $\mathrm{G}_{1}$ & $\mathrm{G}_{\mathrm{s}}$ & $\mathrm{G}_{1}$ & $\mathrm{G}_{\mathrm{s}}$ & $\mathrm{G}_{1}$ & $\mathrm{G}_{\mathrm{s}}$ & $\mathrm{G}_{1}$ & $\mathrm{G}_{\mathrm{s}}$ & $\mathrm{G}_{1}$ & $\mathrm{G}_{\mathrm{s}}$ \\
\hline \hline 61 & 29.1 & 50.5 & 25.4 & 47.9 & 23.1 & 44.9 & 21.9 & 42.6 & 21.2 & 42.7 \\
\hline 25 & 35.0 & 46.3 & 32.2 & 42.5 & 27.8 & 38.2 & 25.4 & 34.9 & 24.1 & 32.6 \\
\hline 3.2 & 41.7 & 41.6 & 37.2 & 38.2 & 32.6 & 33.2 & 28.3 & 29.0 & 24.7 & 26.0 \\
\hline
\end{tabular}


a)

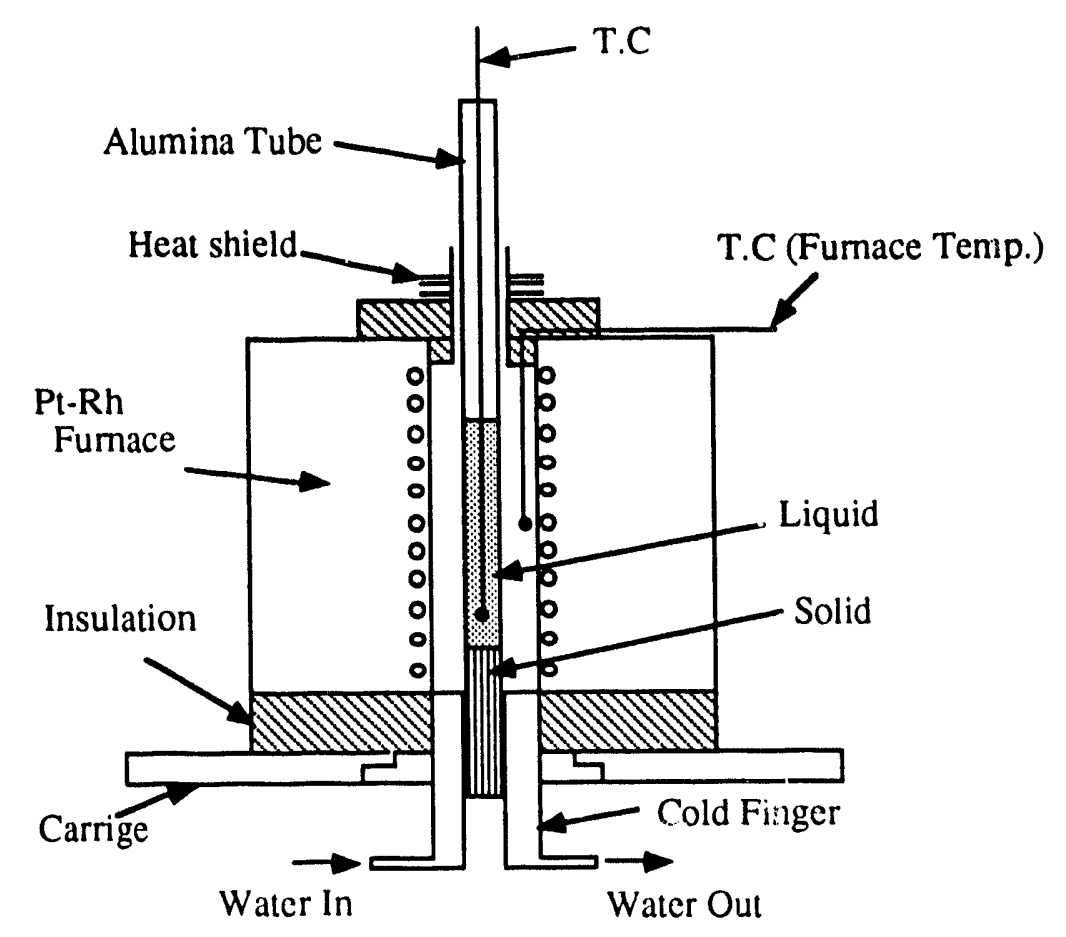

b)

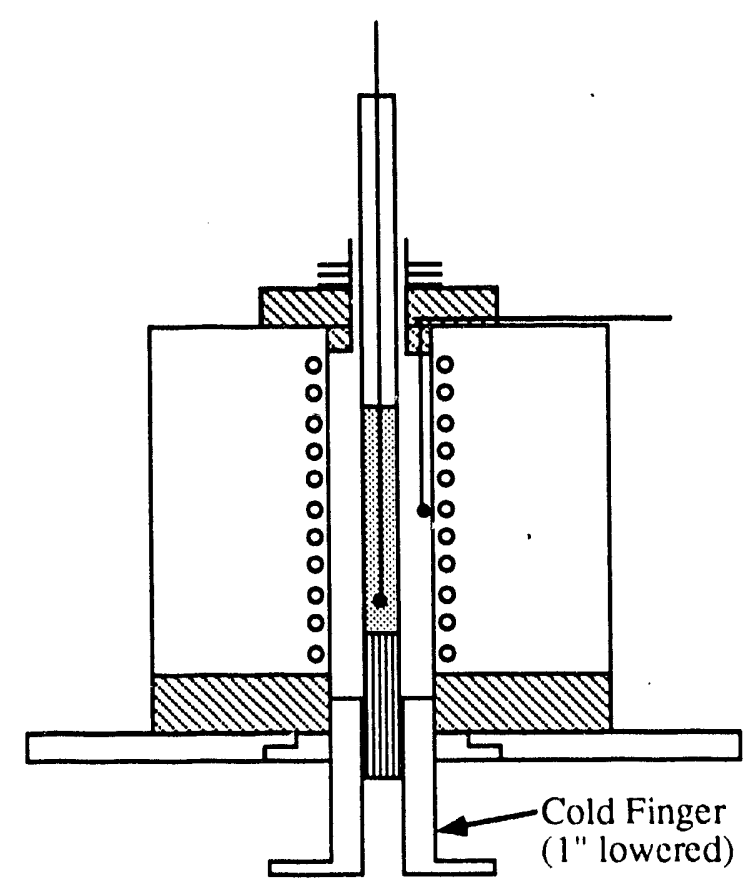

Figure 1. Schematics of the directional solidification apparatus used in this study and the positions of the cold finger. a) Normal position of the cold finger and b) position of the 1 inch lowered cold finger to get a lower temperature gradient 
It was found that the latent he: played an important role in controlling the temperature gradient at a given rate. The break points in the solid-liquid interface are less sharp at low rates and more sharp at high rates due to the latent heat. The $G_{1}$ was decreased and the $G_{s}$ was increased with increasing velocities, as shown in Figure 2.

When the unidirectional heat flow is assumed, the heat flow balance at the solid liquid interface shows;

$$
\begin{array}{ll}
K_{s} G_{S}=K_{l} G_{l}+L V & \text { (1) } \\
G_{l}=\left(K_{S} / K_{l}\right) G_{s}-L V / K_{l} & (1 \mathrm{a}), G_{S}=\left(K_{l} / K_{S}\right) G_{l}+L V / K_{s}
\end{array}
$$

where $\mathrm{K}_{\mathrm{S}}$ and $\mathrm{K}_{\mathrm{l}}$ are thermal conductivities of solid and liquid,

$\mathrm{G}_{\mathrm{S}}$ and $\mathrm{G}_{\mathrm{l}}$ are temperature gradients of solid and liquid, $\mathrm{L}$ is latent heat per unit volume, and $\mathrm{V}$ is velocity.

The Eq(1) shows that $K_{S} / K_{l}$ can be obtained by the $\left(G_{l} / G_{s}\right)$ at $V=0$, and the $E q(1)$ can be expressed as;

$$
\mathrm{K}_{\mathrm{I}}=\frac{\mathrm{L}}{\left\{\left(\frac{\mathrm{K}_{\mathrm{s}}}{\mathrm{K}_{\mathrm{I}}}\right)\left(\frac{\mathrm{G}_{\mathrm{s}}}{\mathrm{V}}\right)-\left(\frac{\mathrm{G}_{\mathrm{I}}}{\mathrm{V}}\right)\right\}}
$$

Also, because the $G_{s}$ and $G_{1}$ have linear relationship with $V$ (the slopes of the $G_{s}$ and $V$ and the $G_{l}$ and $V$ are constant, as shown in Fig. 2) $G_{l}$ and $G_{S}$ can be expressed as;

$$
\mathrm{G}_{1}=\mathrm{Go}_{1}+\mathrm{m}_{\mathrm{l}} \mathrm{V} \quad(3 \mathrm{a}) \quad \text { and } \quad \mathrm{G}_{\mathrm{s}}=\mathrm{Go}_{\mathrm{s}}+\mathrm{m}_{\mathrm{s}} \mathrm{V}
$$

where $\mathrm{G}^{\circ}$ and $\mathrm{G}_{\mathrm{s}}^{\mathrm{s}}$ are the gradients of liquid and solid at $\mathrm{V}=0$ and $m_{I}$ and $m_{S}$ are the slopes of $G$ and $V$ in the liquid and solid.

The Eq.(2) can be changed to

$$
K_{1}=\frac{L}{\left\{\frac{K_{s}}{K_{1}}\left(\frac{G_{s}^{o}}{V}+m_{s}\right)-\left(\frac{G_{1}^{o}}{V}+m_{1}\right)\right\}}
$$

The Eq (4) can be simplified by using $\mathrm{G}_{1}^{O_{1}}=\left(\mathrm{K}_{\sqrt{ }} / \mathrm{K}_{1}\right) \mathrm{G}_{1}^{\circ}$ at $\mathrm{V}=0$ as;

$$
\mathrm{K}_{\mathrm{l}}=\mathrm{L} /\left\{\left(\mathrm{K}_{\mathrm{s}} / \mathrm{K}_{1}\right) \mathrm{m}_{\mathrm{s}}-\mathrm{m}_{\mathrm{l}}\right\} \text { at } \mathrm{V}=0
$$


a)
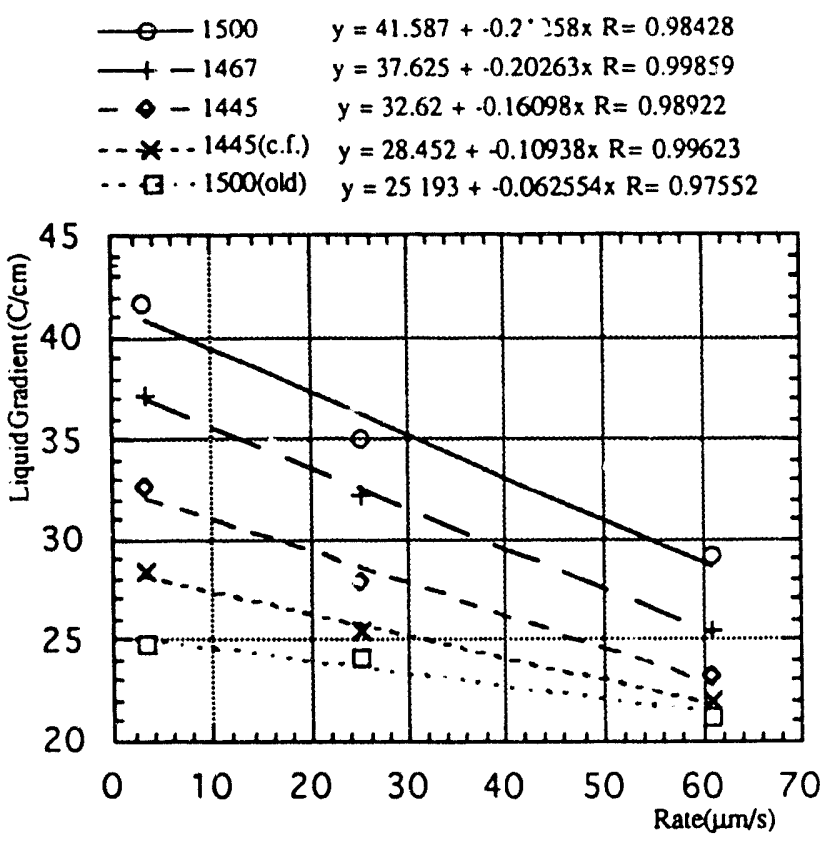

b)

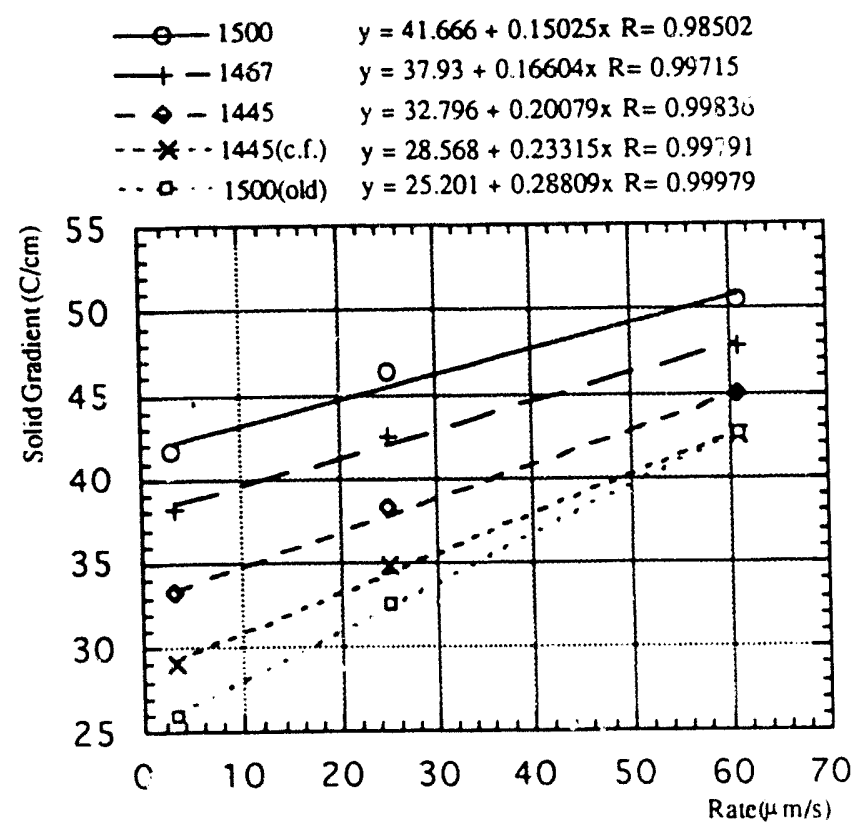

Figure 2. Temperature gradient changes according to the solidification rates in different furnace conditions. a) The liquid gradient and b) the solid gradient 
The $K_{I}$ and $K_{S}$ values may be evaluated from equation (5) if the $G_{S}$ and $G_{l}$ with $V$ are obtained experimentally. The $m_{s}$ and $m_{l}$ values were obtained from the slopes of Figure 2 and the $\left(\mathrm{K}_{\mathrm{S}} / \mathrm{K}_{\mathrm{l}}\right)$ was also obtained from the extrapolated values of $\mathrm{G}_{l}$ and $\mathrm{G}_{\mathrm{S}}$ at $\mathrm{V}=0$ in Figure 2 . When the melting temperature is $1380^{\circ} \mathrm{C}$, the value of the latent heat was assumed $1.653 \times 10^{10} \mathrm{erg} / \mathrm{cm}^{3}$ from ref.[2] and [3]. Table 2 shows the calculated thermal conductivities at the given furnace temperature by $\mathrm{Eq}(5)$. The expected $\mathrm{K}_{l}$ and $\mathrm{K}_{\mathrm{s}}$ are almost the same as $0.46 \mathrm{~W} / \mathrm{cm}^{\circ}{ }^{\circ} \mathrm{C}$ at about $1380^{\circ} \mathrm{C}$ (Liquid; $1380-1400^{\circ} \mathrm{C}$, and Solid; $1360-1380{ }^{\circ} \mathrm{C}$ ). The $\mathrm{K}_{\mathrm{s}}$ was measured from room temperature to $727^{\circ} \mathrm{C}$ in ref [4]. It was about $0.36-0.33 \mathrm{~W} / \mathrm{cm}-{ }^{\circ} \mathrm{C}$ at $727^{\circ} \mathrm{C}$ and these values are in the reasonable range with the expected value of $0.46 \mathrm{~W} / \mathrm{cm}-{ }^{\circ} \mathrm{C}$ at about $1380{ }^{\circ} \mathrm{C}$.

Table 2. Estimated thermal conductivities from equation (5) and the inv v. ved parameters

\begin{tabular}{c|c|c|c|c|c}
\hline Furnace Temp. $\left({ }^{\circ} \mathrm{C}\right)$ & $\mathrm{K}_{\mathrm{S}} / \mathrm{K}_{\mathrm{l}}$ & $\mathrm{m}_{1}\left({ }^{\circ} \mathrm{Cs} / \mathrm{cm}^{2}\right)$ & $\mathrm{m}_{\mathrm{S}}\left({ }^{\circ} \mathrm{Cs} / \mathrm{cm}^{2}\right)$ & $\mathrm{K}_{1}\left(\mathrm{~J} / \mathrm{cm}-{ }^{\circ} \mathrm{C}\right)$ & $\mathrm{K}_{\mathrm{S}}\left(\mathrm{J} / \mathrm{cm}-{ }^{\circ} \mathrm{C}\right)$ \\
\hline \hline 1509 & 1.00 & -2125.8 & 1502.5 & 0.44 & 0.44 \\
1467 & 0.99 & -2026.3 & 1660.4 & 0.46 & 0.46 \\
1445 & 1.00 & -1609.8 & 2007.9 & 0.46 & 0.46 \\
1445 (low c.f.) & 1.00 & -1093.8 & 2331.5 & 0.48 & 0.48 \\
1500 (old) & 1.00 & -625.5 & 2880.9 & 0.47 & 0.47 \\
\hline
\end{tabular}

There are few measurements of the high temperature thermal condutivities, especially in the liquid, because it is difficult to measure and control the heat fluxes experimentally at the high temperature. The present experiments show that the high temperature thermal conductivities of the solid and liquid can be measured reasonably well by the directional solidification technique.

As a conclusion, the therma conductivity of $\mathrm{Ni}_{3} \mathrm{Al}$ was determined by a heat flux balance in directional solidification experiments. The thermal conductivities of the liquid and 
solid were found to be essencially equal, with a value of $0.46 \mathrm{~W} / \mathrm{cm}-{ }^{\circ} \mathrm{C}$ at the melting point of $\mathrm{Ni}{ }_{3} \mathrm{Al}$.

\section{References}

1. Jamgotchian, H.; Billia, B.; Capella, L. J. Cry. Growth, 1983, 64, 338.

2. Quested, P.N.; McLean, M. Mat. Sci.Eng., 1984, 65, 171.

3. Kim, H.K.; Earthman, J.C.; Levernia, E.J. Acta Met., 1992,40(4), 637.

4. Williams, R.K.; Graves, R.S.; Weaver, F.J. J. Appl. Phys., 1987, 61(4), 1486. 

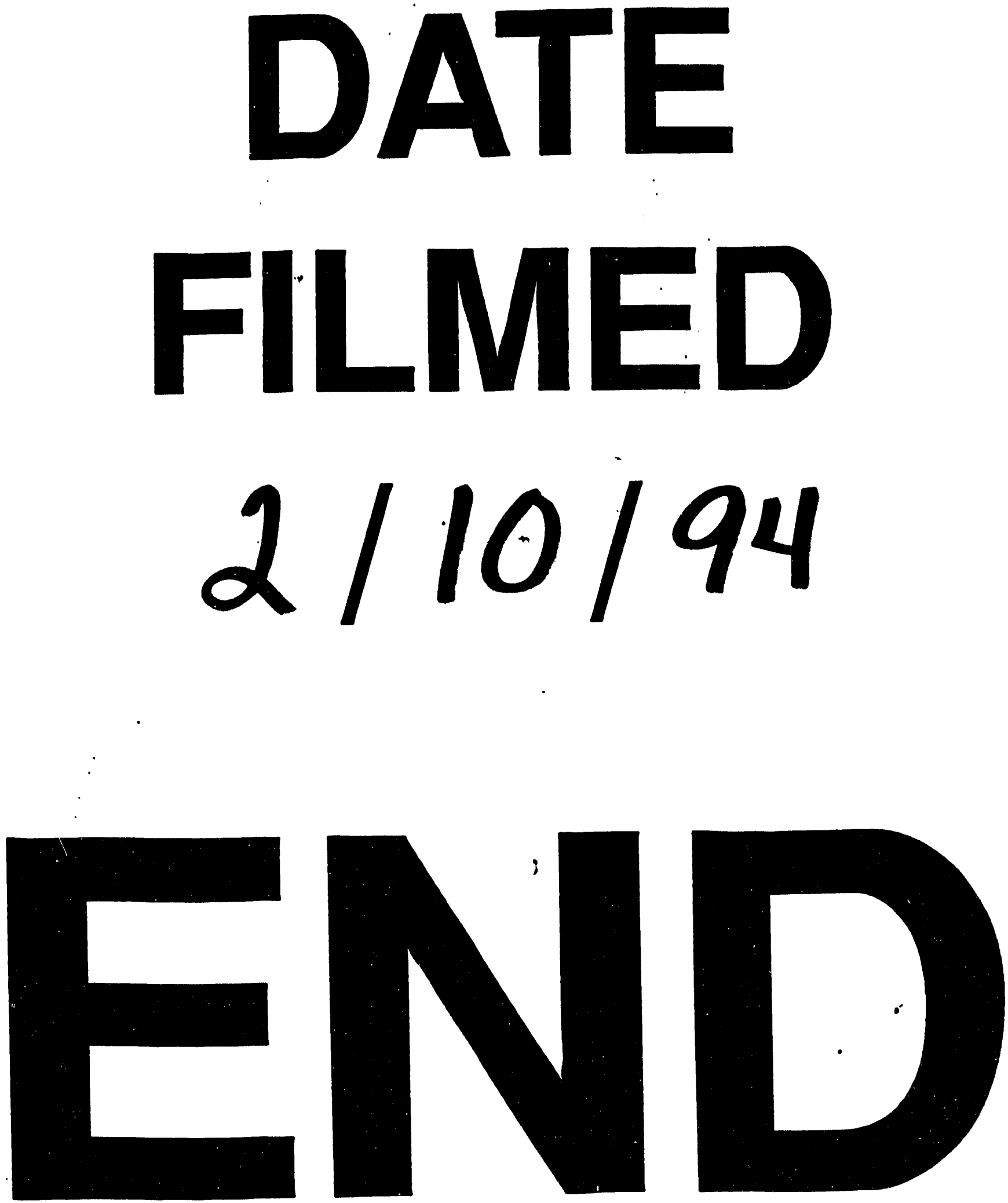
